Final Report • November 2004

\title{
FUNDAMENTAL UNDERSTANDING OF CRACK GROWTH IN STRUCTURAL COMPONENTS OF GENERATION IV SUPERCRITICAL LIGHT WATER REACTORS
}

SRI Project P11489

Contract No. DE-FG07-01SF22332

Prepared by:

Iouri I. Balachov, Senior Research Engineer

Takao Kobayashi, Senior Staff Scientist

Francis Tanzella, Senior Electrochemist Indira Jayaweera, Senior Scientist

Palitha Jayaweera, Senior Electrochemist

Petri Kinnunen, Research Engineer

Martin Bojinov, Senior Material Scientist

Timo Saario, Group Leader

Prepared for:

U.S. Department of Energy

Office of Nuclear Energy, Science \& Technology

19901 Germantown Rd.

Germantown, MD 20874

Attn: Lynn Hall, NE-20

Approved:

Angel Sanjurjo Laboratory Director

Materials Research Laboratory 


\section{REPORT DOCUMENTATION PAGE}

Form Approved

OMB NO. 0704-0188

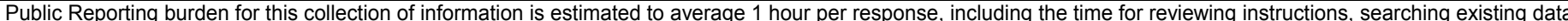

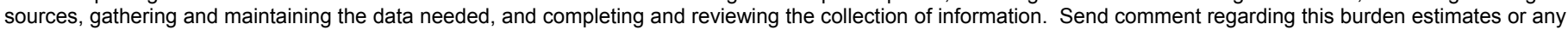

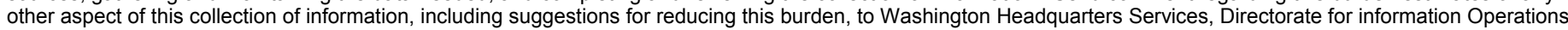

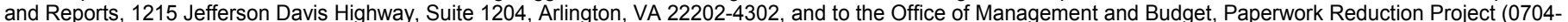
0188,) Washington, DC 20503

\begin{tabular}{l|l|l|l}
\hline 1. AGENCY USE ONLY (Leave Blank) & $\begin{array}{l}2 . \quad \text { REPORT DATE } \\
\text { October } 2004\end{array}$ & $\begin{array}{l}\text { R. REPORT TYPE AND DATES COVERED } \\
\text { Final report: August 2001 - July 2004 }\end{array}$ & $\begin{array}{l}\text { FUNDING NUMBERS } \\
\text { Contract No. DE-FG07-01SF22332 }\end{array}$
\end{tabular}

Generation IV Supercritical Light Water Reactors

AUTHOR(S): Iouri I. Balachov, Takao Kobayashi, Indira Jayaweera, Francis

Tanzella, Palitha Jayaweera, Petri Kinnunen, Martin Bojinov,

Timo Saario

6. PERFORMING ORGANIZATION NAME(S) AND ADDRESS(ES)

SRI International, 333 Ravenswood Avenue, Menlo Park, CA 94025

8. PERFORMING ORGANIZATION

REPORT NUMBER

9. SPONSORING / MONITORING AGENCY NAME(S) AND ADDRESS(ES)

U.S. Department of Energy, Office of Nuclear Energy, Science \& Technology,

19901 Germantown Rd., Germantown, MD 20874

AGENCY REPORT NUMBER

11. SUPPLEMENTARY NOTES

12 a. DISTRIBUTION / AVAILABILITY STATEMENT

12 b. DISTRIBUTION CODE

Approved for public release; distribution unlimited.

13. ABSTRACT (Maximum 200 words)

This work contributes to the design of safe and economical Generation-IV Super-Critical Water Reactors (SCWRs) by providing a basis for selecting structural materials to ensure the functionality of in-vessel components during the entire service life. During the second year of the project, we completed electrochemical characterization of the oxide film properties and investigation of crack initiation and propagation for candidate structural materials steels under supercritical conditions. We ranked candidate alloys against their susceptibility to environmentally assisted degradation based on the in situ data measured with an SRI-designed controlled distance electrochemistry (CDE) arrangement. A correlation between measurable oxide film properties and susceptibility of austenitic steels to environmentally assisted degradation was observed experimentally. One of the major practical results of the present work is the experimentally proven ability of the economical CDE technique to supply in situ data for ranking candidate structural materials for Generation-IV SCWRs. A potential use of the CDE arrangement developed at SRI for building in situ sensors for monitoring water chemistry in the heat transport circuit of Generation-IV SCWRs was evaluated and proved to be feasible.

\begin{tabular}{l|c} 
14. SUBJECT TERMS & 15. NUMBER OF PAGES \\
Generation-IV reactors, supercritical light water reactors, stress corrosion cracking & 159
\end{tabular}

16. PRICE CODE

\begin{tabular}{|c|c|c|}
\hline 17. SECURITY CLASSIFICATION & 18. SECURITY CLASSIFICATION & 19. SECURITY CLASSIFICATION \\
\hline OF REPORT & ON THIS PAGE & OF ABSTRACT \\
\hline UNCLASSIFIED & UNCLASSIFIED & UNCLASSIFIED \\
\hline
\end{tabular}

20. LIMITATION OF ABSTRACT

NSN 7540-01-280-5500

Standard Form 298 (Rev.2-89)

Prescribed by ANSI Std. 239-18

298-102 


\section{CONTENTS}

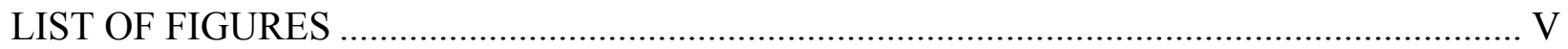

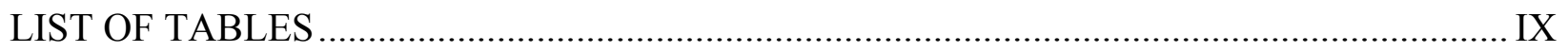

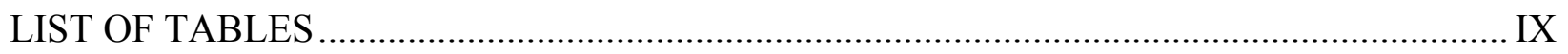

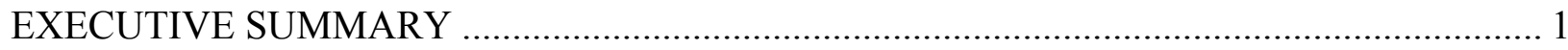

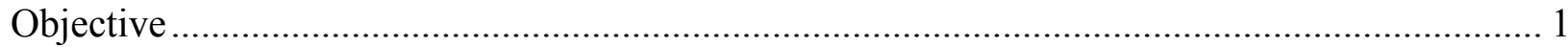

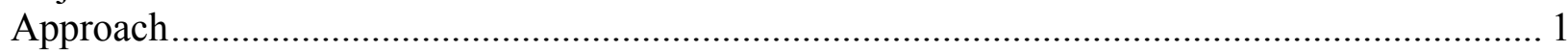

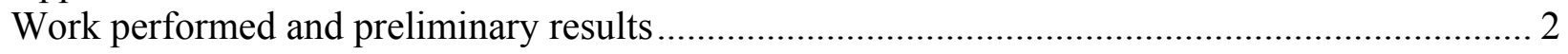

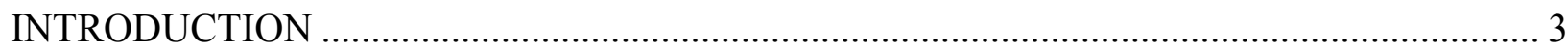

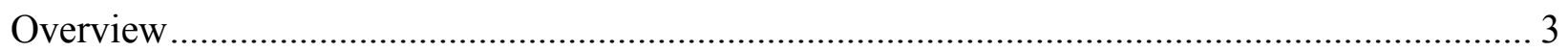

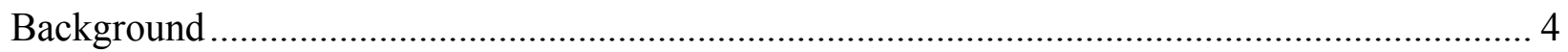

In situ Methods to Study Environmentally Assisted Degradation of Materials ...................... 5

Candidate Materials for SCWR In-Vessel Components ......................................................... 7

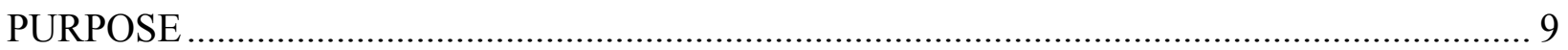

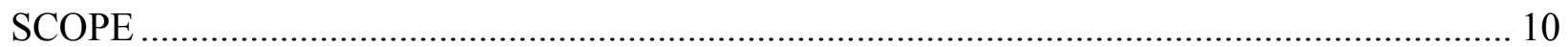

Task 1. Development of a CDE technique for supercritical temperatures ................................... 10

Task 2. An electrochemical characterization of the oxide film properties on candidate materials in supercritical conditions in bulk and within cracks................................................ 10

Task 3. Experimental investigation of crack initiation and propagation and estimation of the

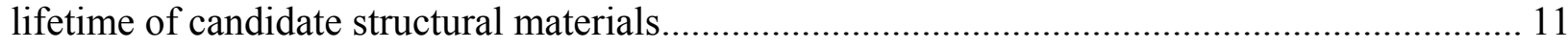

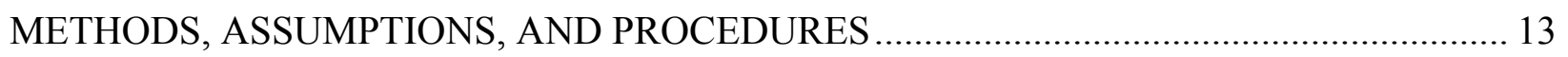

In situ Measurements of the Kinetics of Charge and Mass Transfer in Oxide Films .................... 15

Role of Controlled Distance Electrochemistry in Materials Reliability Assessment ............ 15

Symmetrical and Asymmetrical Configurations for In situ Study of Oxide Films................ 21

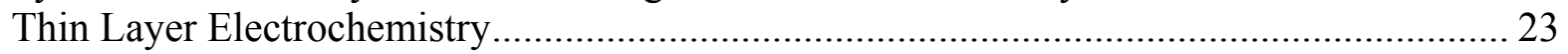

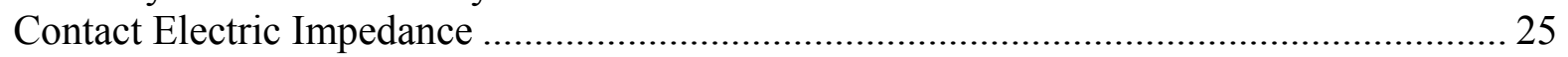

Kinetic Models for the Asymmetrical Configuration .......................................................... 26

Kinetic Models for the Symmetrical Configuration.............................................................. 29

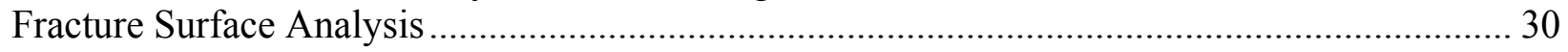

Pretreatment of the Fracture Surfaces ……………………............................................. 34

Obtaining a 3-D Topography Map of the Conjugate Fracture Surfaces ................................. 36

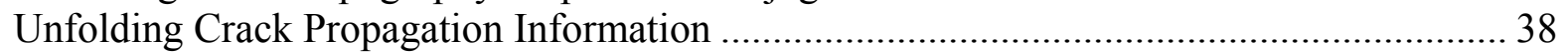

Estimation of Crack Growth Rates................................................................................... 39

Correlation of the Oxide Film Stability and Crack Growth.......................................................... 40

Multi-Disciplinary View of Stress Corrosion Cracking...................................................... 40

Role of Repassivation in Crack Evolution .......................................................................... 42

A Step-Wise Crack Advance Mechanism ....................................................................... 44

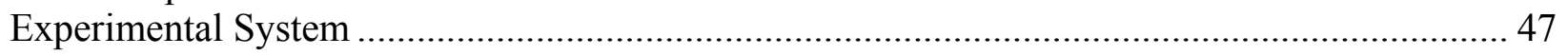

CDE Arrangement for Supercritical Water........................................................................ 47

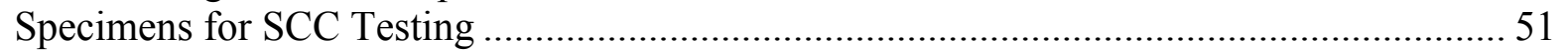

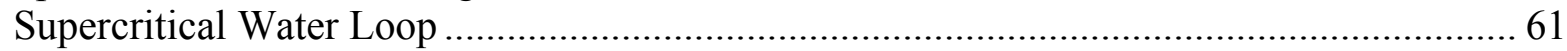

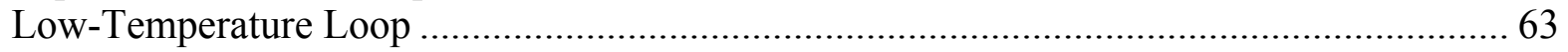

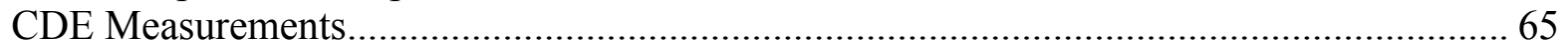




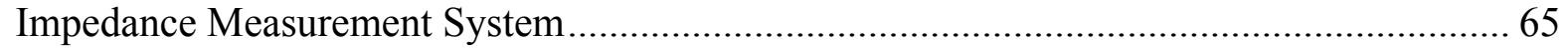

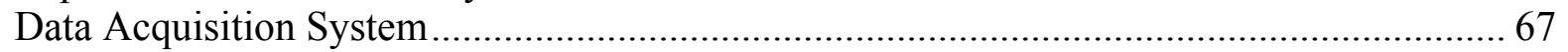

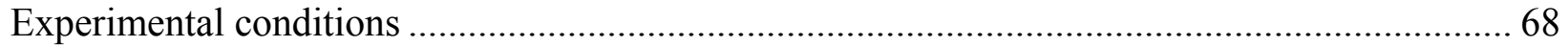

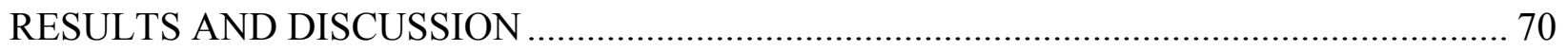

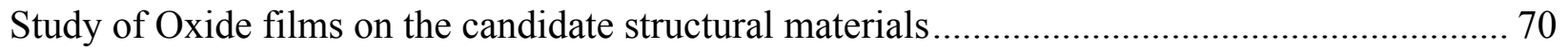

Validation of the Measured Impedance Spectra ........................................................... 70

Collection of data on the charge and mass transfer properties of the oxide films ............... 75

Validation of kinetic parameters of the charge and mass transfer processes in the oxide

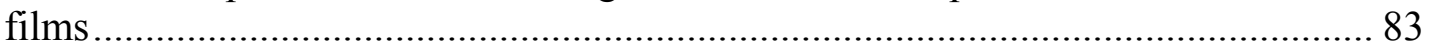

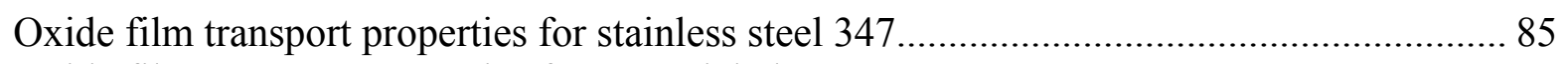

Oxide film transport properties for pure nickel......................................................... 92

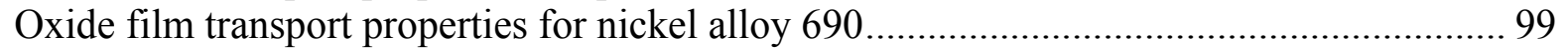

Oxide film transport properties for alloy T91 ............................................................ 103

Oxide film transport properties for alloy HT9 ............................................................. 106

In situ Monitoring of Water Chemistry in Generation-IV SCWRs ................................. 112

Chemical composition and thickness of the oxide films............................................ 114

Study of crack propagation in internally loaded specimens ............................................. 120

Post Examination of the Fracture Surfaces ............................................................... 120

Estimated Crack Initiation Times.................................................................................. 133

Estimated Crack Growth Rates ................................................................................ 134

Ranking Materials against Susceptibility to Environmentally Assisted Degradation ......... 136

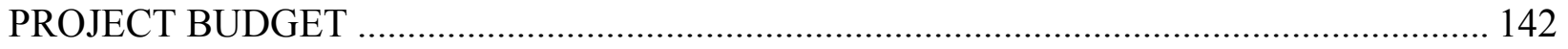

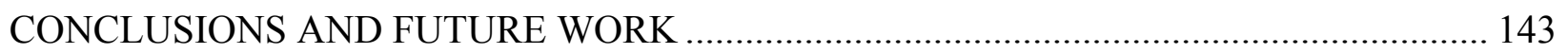

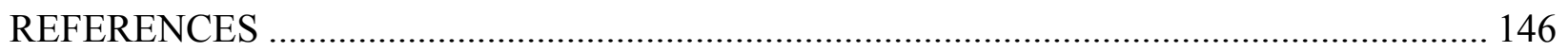




\section{LIST OF FIGURES}

Figure 1. SRI's approach to studying the fundamentals of material stability in supercritical water. ................6

Figure 2. Controlled Distance Electrochemistry measurements: (1) specimens are in contact for CER and CEI measurements, (2) specimens are exposed to the environment, (3) oxidation takes place, (4) specimens are in contact for CER and CEI measurements......................................................... 16

Figure 3. A scheme of the controlled-distance electrochemistry (CDE) arrangement and its different applications. Alloy 82 in the presence of 0 (top) and $10000 \mathrm{ppb}$ (bottom) sulfate ions in simulated crack conditions at $273^{\circ} \mathrm{C}$ (symbols: experimental points; lines: fitted results).

Figure 5. The value of the resistance $\mathrm{R}_{\mathrm{D}}$ for the transport of ions or ionic defects through the oxide film on AISI $316 \mathrm{NG}$ and Inconel alloys 182 and 82 as a function of sulfate ion content in simulated crack chemistry conditions.

Figure 6. A typical TLEC impedance spectrum of the oxide film formed in supercritical water: (a) all

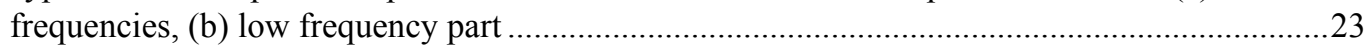

Figure 7. Equivalent electrical circuit for metal/film/electrolyte (asymmetrical) configuration. ....................25

Figure 8. Typical CEI impedance spectrum of the oxide film formed in supercritical water.........................25

Figure 9. Use of a confocal-optics-based laser microscope (SLM) in the FRASTA technique makes it possible to characterize surface topography quickly and accurately (left top and bottom). FRASTA results are presented as fractured area projection plots, which provide information on microcrack initiation sites and projected areas of micro/macrocracks; and as cross-sectional plots, which show the microcracks in profile, the macrocrack tip opening angle and displacement, and the amount of inelastic deformation necessary before fracture [60].

Figure 10. Series of fractured area projection plots for Type 304 CERT specimens tested in simulated pure BWR water (left column) and in BWR with 1 ppm sulfuric acid (right column). Black area indicates intact material; white area, cracks [60].

Figure 11. SEM photographs of the same fracture surface area before and after electrochemical cleaning...36

Figure 12. Higher magnification SEM photograph of the oxide-covered fracture surface. .............................36

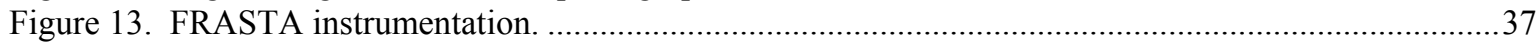

Figure 14. An example of the 3-D topography map of the fracture surface................................................38

Figure 15. Fractured Area Projection Plot superimposed on an SEM image of the fracture surface..............39

Figure 16. Impact of the oxide film stability on the SCC crack propagation. "Stronger" denotes more stable film (higher resistivity to charge and mass transfer) ....................................................................4

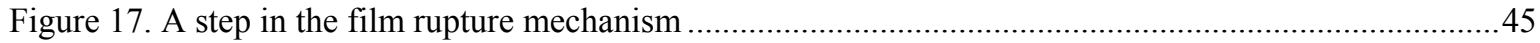

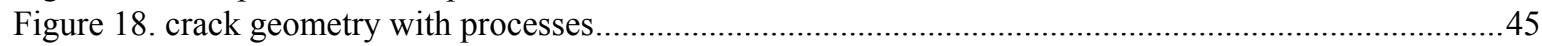

Figure 19. Arrangement for controlled distance electrochemistry studies at supercritical temperatures. (a) general view and (b) close view showing guiding tube, specimen holders, and CDE specimens.48

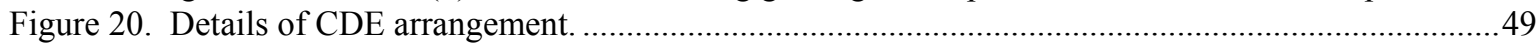

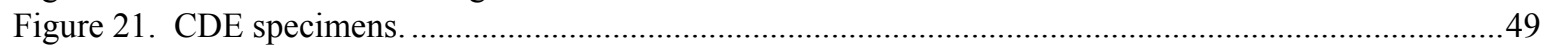

Figure 22. Electrical connection of the CDE specimens. ...................................................................50

Figure 23. Variation of the contact electric resistance with distance between working surface of CDE

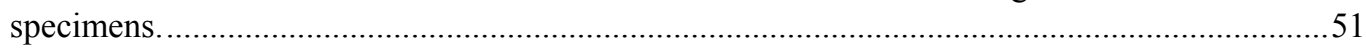

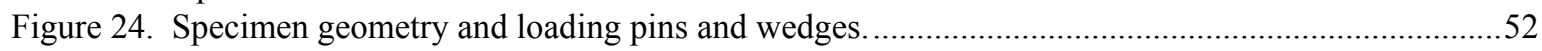

Figure 25 Mesh used in the finite element analysis of the center notched disc..........................................53

Figure 26. Effective stress distribution in the center notch disc under fixed pin-displacement loading. .........53

Figure 27. Variation of stress intensity as a function of crack length under a unit fixed displacement. ..........54

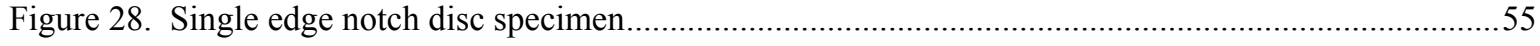


Figure 29. Meshes used in the finite element analysis of the single edge notched disc and deformed geometry of the disc under fixed pin displacement of 0.005 inches. The crack length was 0.30 inches. (a) finite element meshes in the disc; (b) deformed geometry under fixed pin displacement of 0.005 inches.

Figure 30. Effective stress distribution in the disc under fixed pin displacement of 0.005 inches at the crack length $\mathrm{a}=0.30$ inches.

Figure 31. Stress intensity factor variation as a function of crack length under fixed pin displacement loading condition. Three cases of pin-displacement are shown...............................................................57

Figure 32. Experimental setup showing two electro-optical displacement transducers. ...............................59

Figure 33. Macro photograph of the side of the disc specimen showing the fatigue precrack extending from the electron beam notched tip. ...............................................................................................5

Figure 34. Wedge loaded single-edge-notch disc specimen ready to be placed in the autoclave. ..................60

Figure 35. General view of the experimental system: supercritical test reactors (1), the high-pressure loop (2),

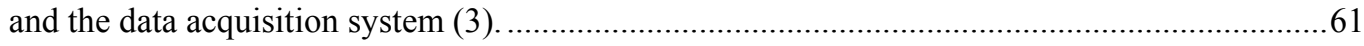

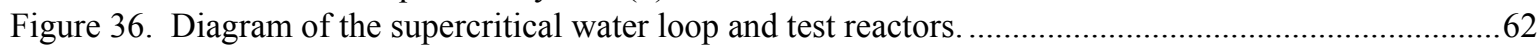

Figure 37. Close view of the APSS reactor (left) and CDE reactor (right) ..................................................63

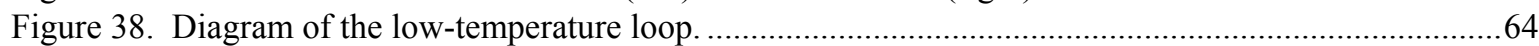

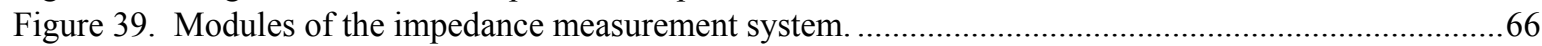

Figure 40. Display of the impedance measurement and analysis system. ...................................................66

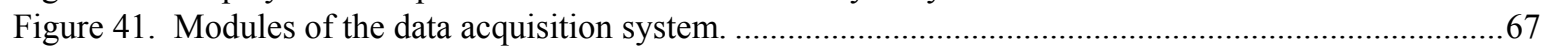

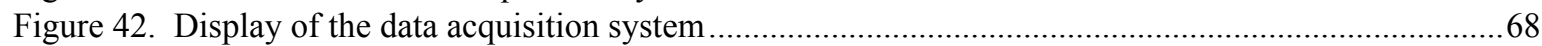

Figure 43. TLEC spectra measured for gradually increasing amplitude of the AC signal............................71

Figure 44. Average impedance and deviations for a set of measurements with AC signal amplitudes ranging from 100 to $400 \mathrm{mV}$

Figure 45. Percentage deviation in the impedance measured with an AC signal ranging from 100 to $400 \mathrm{mV}$.

Figure 46. Variations in the real and imaginary part of the CDE impedance measurements for a single frequency $(1 \mathrm{~Hz})$ with amplitude of the AC signal. ................................................................... 73

Figure 47. K-K transform causality test results for impedance spectra measured for asymmetrical (a) and

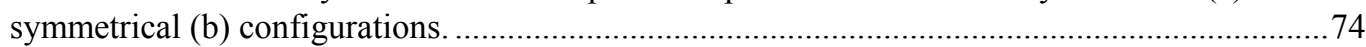

Figure 48. TLEC spectra measured in symmetrical configuration: the entire frequency range (a) and the low-

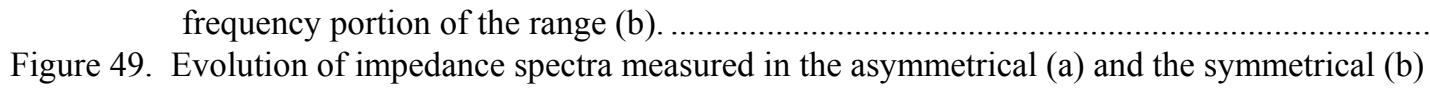
configuration with formation and growth of the oxide film. ...................................................78

Figure 50. Equivalent circuits of the transfer functions of the oxide films: Model 1 (a) and Model 2 (b)......80

Figure 51. Equivalent circuits for the complete system: Model $1(\mathrm{a}, \mathrm{b})$ and Model 2 (c, d)........................80

Figure 52. Comparison of measured and calculated impedance spectra for the asymmetrical configuration. 81

Figure 53. Comparison of measured and calculated impedance spectra for the symmetrical configuration...82

Figure 54. TLEC impedance spectra for AISI 316 at $150^{\circ} \mathrm{C}$ and distances of $50 \mu \mathrm{m}$ (a) and at $300^{\circ} \mathrm{C}$ and

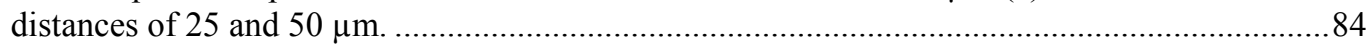

Figure 55. Illustration of "critical level" of oxidizing conditions in supercritical water on electronic properties of the oxide film formed on 347 stainless steel.

Figure 56. Evolution of the phase angle during CEI measurement of the oxide film growth on 347 stainless steel in supercritical water. Concentration of dissolved oxygen is $100 \mathrm{ppb}$.................................86

Figure 57. Impedance parameters of the oxide film formed on 347 stainless steel exposed to supercritical water: charge transfer resistance, diffusion (Warburg) resistance, contact electric resistance (CER) and polarization resistance $\left(\mathrm{R}_{\mathrm{p}}\right)$. Concentrations of dissolved oxygen: (a) - $100 \mathrm{ppb}$, (b) -400 $\mathrm{ppb}$, and (c) $-1400 \mathrm{ppb}$.

Figure 58. Effect of supercritical water oxidizing conditions on Young's impedance of the oxide film growing on 347 stainless steel. Concentrations of dissolved oxygen: (a) - $100 \mathrm{ppb}$, (b) - $400 \mathrm{ppb}$, and (c) $-1400 \mathrm{ppb}$.

Figure 59. Oxide film (layer in which point defect transport occurs) growth on 347 stainless steel exposed to supercritical water. Concentrations of dissolved oxygen: (a) - $100 \mathrm{ppb}$, (b) $-400 \mathrm{ppb}$, and (c) 1400 ppb. 
Figure 60. Strength of the electric field across the oxide film formed on 347 stainless steel exposed to supercritical water. Concentrations of dissolved oxygen: (a) - $100 \mathrm{ppb}$, (b) - $400 \mathrm{ppb}$, and (c) -

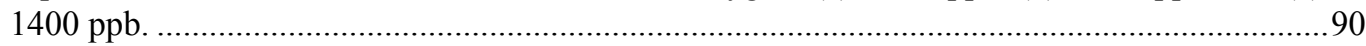

Figure 61. Ionic $\left(D_{i}\right)$ and electronic $\left(D_{e}\right)$ diffusion coefficients of the oxide film formed on 347 stainless steel exposed to supercritical water. Concentrations of dissolved oxygen: (a) $-100 \mathrm{ppb}$, (b) $-400 \mathrm{ppb}$, and $(\mathrm{c})-1400 \mathrm{ppb}$.

Figure 62. Electronic properties of the oxide film formed on pure nickel in supercritical water at various levels of dissolved oxygen.

Figure 63 Evolution of the impedance spectra for the oxide film growing on pure nickel in supercritical water with $500 \mathrm{ppb}$ of dissolved oxygen.

Figure 64. Impedance parameters of the oxide film formed on pure nickel exposed to supercritical water: charge transfer resistance, diffusion (Warburg) resistance, contact electric resistance (CER) and polarization resistance $\left(\mathrm{R}_{\mathrm{p}}\right)$. Concentrations of dissolved oxygen: (a) $-100 \mathrm{ppb}$, (b) - $500 \mathrm{ppb}$, and (c) $-2000 \mathrm{ppb}$.

Figure 65. Effect of supercritical water oxidizing conditions on Young's impedance of the oxide film growing on pure nickel. Concentrations of dissolved oxygen: (a) - $100 \mathrm{ppb}$, (b) $-500 \mathrm{ppb}$, and (c) -2000 ppb.

Figure 66. Oxide film (layer in which point defect transport occurs) growth on pure nickel exposed to supercritical water. Concentrations of dissolved oxygen: (a) - $100 \mathrm{ppb}$, (b) - $500 \mathrm{ppb}$, and (c) $2000 \mathrm{ppb}$.

Figure 67. Strength of the electric field across the oxide film formed on pure nickel exposed to supercritical water. Concentrations of dissolved oxygen: (a) $-100 \mathrm{ppb}$, (b) - $500 \mathrm{ppb}$, and (c) - $2000 \mathrm{ppb} . .97$

Figure 68. Ionic $\left(D_{i}\right)$ and electronic $\left(D_{e}\right)$ diffusion coefficients of the oxide film formed on pure nickel exposed to supercritical water. Concentrations of dissolved oxygen: (a) $-100 \mathrm{ppb}$, (b) $-500 \mathrm{ppb}$, and (c) $-2000 \mathrm{ppb}$.

Figure 69. Electronic properties of the oxide film formed on alloy 690 in supercritical water at various levels of dissolved oxygen.

Figure 70. Evolution of the impedance spectra for the oxide film growing on A690 in supercritical water with $50 \mathrm{ppb}$ of dissolved oxygen: (a) - modulus of impedance, (b) - phase angle.

Figure 71. Impedance parameters of the oxide film formed on pure nickel exposed to supercritical water: charge transfer resistance, diffusion (Warburg) resistance, contact electric resistance (CER) and polarization resistance $\left(\mathrm{R}_{\mathrm{p}}\right)$. Concentration of dissolved oxygen $100 \mathrm{ppb}$

Figure 72. Evolution of Young's impedance of the oxide film growing on alloy 690 in supercritical water. Concentration of dissolved oxygen $100 \mathrm{ppb}$.

Figure 73. Oxide film (layer in which point defect transport occurs) growth on alloy 690 exposed to supercritical water. Concentration of dissolved oxygen $100 \mathrm{ppb}$.

Figure 74. Strength of the electric field across the oxide film formed on alloy 690 exposed to supercritical water. Concentration of dissolved oxygen $100 \mathrm{ppb}$.

Figure 75. Ionic $\left(D_{i}\right)$ and electronic $\left(D_{e}\right)$ diffusion coefficients of the oxide film formed on alloy 690 exposed to supercritical water. Concentration of dissolved oxygen $100 \mathrm{ppb}$.

Figure 76. Electronic properties of the oxide film formed on alloy T91 in supercritical water at various levels of dissolved oxygen

Figure 77. Evolution of CEI spectrum for oxide film growing on alloy T91 exposed to supercritical water with $5000 \mathrm{ppb}$ of dissolved oxygen.

Figure 78. Evolution of CEI spectrum for oxide film growing on alloy T91 exposed to supercritical water with $30 \mathrm{ppb}$ of dissolved oxygen: (a) - phase angle, (b) - modulus

Figure 79. Electronic properties of the oxide film formed on alloy T91 in supercritical water (a) at various levels of dissolved oxygen (b)

Figure 80. Evolution of CEI spectrum for oxide film growing on alloy HT9 exposed to supercritical water with $5000 \mathrm{ppb}$ of dissolved oxygen: (a) - phase angle, (b) - modulus

Figure 81. Evolution of CEI spectrum for oxide film growing on alloy HT9 exposed to supercritical water with 5000 ppb of dissolved oxygen: (a) - phase angle, (b) - modulus. Data from Figure 80 for longer exposure times only 
Figure 82. Evolution of CEI spectrum for oxide film growing on alloy HT9 exposed to supercritical water with $50 \mathrm{ppb}$ of dissolved oxygen: (a) - modulus and phase angle, (b) - phase angle for frequency < $1 \mathrm{kHz}$. .110

Figure 83. Evolution of TLEC spectrum for oxide film growing on alloy HT9 exposed to supercritical water with 5000 ppb of dissolved oxygen: (a) modulus and phase angle, (b) - Nyquist plot...... .111

Figure 84. Evolution of TLEC spectrum for oxide film growing on alloy HT9 exposed to supercritical water with $50 \mathrm{ppb}$ of dissolved oxygen....

Figure 85. Film response to change in oxidizing conditions. .113

Figure 86. Monitoring resistance of the water between CDE electrodes during heating from room temperature to supercritical temperature.

Figure 87. Low-magnification image of SS316 specimens: bottom specimen (a), top specimen (b). Arrows point to EDX analysis spots.

Figure 88. High-magnification image of SS316 specimens: bottom specimen (a), top specimen (b). Arrows

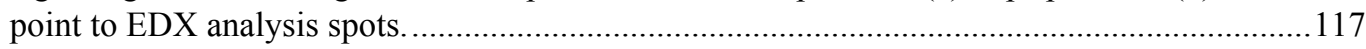

Figure 89. Composition of the oxide films on the SS316 specimen.........................................................118

Figure 90. Surface averaged composition of the oxide film...................................................................118

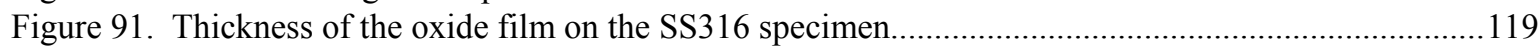

Figure 92. Cr profile in the oxide film formed on SS3 16 at $400^{\circ} \mathrm{C}$ and $3500 \mathrm{psi}$.................................119

Figure 93. SSC specimen removed from the supercritical reactor. ......................................................... 120

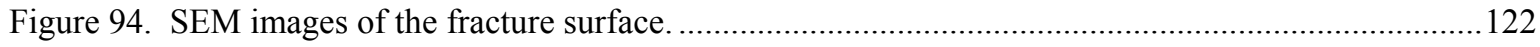

Figure 95. High-magnification SEM image of the fracture surface showing "time marks."........................123

Figure 96. Contrast and gray-scale topography images of conjugate surfaces............................................124

Figure 97. Perspective view of fracture surfaces. fracture surfaces. ...........................................................126

Figure 98. A series of FAPPs showing the crack growth processes. The plots show hesitation in crack front movement at some locations.

Figure 99. Contrast images of conjugate surfaces and a corresponding SEM photograph. (Surface B image was flipped to enable comparison of images.) (........................................................................... 128

Figure 100. Superposition of selected FAPPs on the SEM photograph showing the correlation of interconnected placed (red areas) in FAPPs with whitish areas on SEM. ................................129

Figure 101. A series of FAPPs for chemically cleaned fracture surfaces....................................................130

Figure 102. Fractured area increase curve as a function of conjugate surface spacing...............................131

Figure 103. Superposition of FAPP at Point A over the contrast image and the corresponding XSP.........132

Figure 104. Superposition of FAPP at Point B over the contrast image and the corresponding XSP..........133

Figure 105. Estimated crack initiation times for austenitic stainless steels ..................................................134

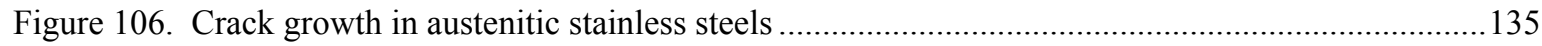

Figure 107. Crack growth rates for austenitic stainless steels ...................................................................136

Figure 108. Transport properties of the oxide films (a) and crack growth rates (b) relative to SS304........137

Figure 109. Repassivation time of SS347, pure nickel and alloy 690................................................138

Figure 110. Crack growth rate decrease due to repassivation for SS347 and alloy A690: (a) without considering oxide film stability and (b) with exponent in repassivation term proportional to oxide film stability.

Figure 111. Crack growth in candidate structural materials under low, medium, and high oxidizing conditions

.

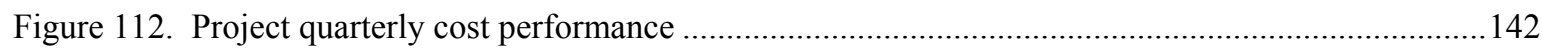




\section{LIST OF TABLES}

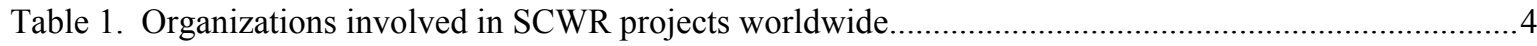

Table 2. Operation modes of the test reactors in the supercritical water loop.................................................62

Table 3. Chemical composition of candidate and tested materials.............................................................69

Table 4. Average Warburg impedance parameters obtained from simulations and calculations. ....................84 


\section{EXECUTIVE SUMMARY}

This report describes the approach, methods used, and experimental and theoretical results of the work SRI International (SRI) performed under NERI Project 1-130, "Fundamental Understanding of Crack Growth in Structural Components of Generation IV Supercritical Light Water Reactors."

\section{OBJECTIVE}

The project objective is to develop fundamental understanding of the corrosion and stress corrosion cracking (SCC) behavior of alloys in supercritical water. The theoretical and experimental results of this project will aid to this dominant feature of the international efforts to select structural materials for SCWR

\section{APPROACH}

The NERI project is drawing on the following unique combination of advanced materials characterization techniques to meet the objective:

Screening alloys. In situ characterization of the oxide films formed on candidate structural materials allows ranking materials according to their susceptibility to general and localized forms of environmentally assisted degradation.

Understanding basic phenomena. Kinetic parameters of the charge and mass transport processes in the metal/oxide film/supercritical water system are derived from in situ oxide film studies to understand and quantify the rate-controlling processes and, eventually, the fundamentals of the metal oxidation phenomenon at supercritical temperatures.

Providing a basis for the development of new alloys. Integration of the in situ experimental information on oxide film resistance to charge and mass transport for alloys of various compositions, along with fracture surface analysis and current theoretical understanding of the relationship between properties of the oxide films and stability of materials, is expected to aid in material selection and the development process. It will do so by providing recommendations for chemical compositions of the alloys in regard to their expected locations in the reactor (or more specifically, in regard to local temperature and oxidizing conditions). 


\section{WORK PERFORMED AND PRELIMINARY RESULTS}

During this project, SRI completed the electrochemical characterization of the oxide film properties and the investigation of crack initiation and propagation for the following group of candidate structural materials — austenitic stainless steels, nickel alloys, and ferritic-martensitic steels under supercritical conditions.

A summary of SRI's major accomplishments during this project follows:

- Systematic experimental data on the charge and mass transfer properties of the oxide films were obtained.

- Kinetic parameters of the charge and mass transfer processes in the oxide determined from controlled distance electrochemistry (CDE) measurements were in good agreement with independent results obtained at VTT.

- A set of kinetic parameters, which describe transport processes, was defined.

- The first group of candidate structural materials (austenitic stainless steels SS304, SS316, and SS347) was ranked in regard to their susceptibility to environmentally assisted degradation based on the in situ data on film stability parameters (or film transport properties) measured with the SRI-designed CDE arrangement.

- Resistance to both electronic and ionic charge carries was observed to be the lowest for SS304 and the highest for SS347, with SS316 being between the two.

- A correlation between measurable oxide film properties and susceptibility of austenitic steels to environmentally assisted degradation was observed experimentally. Experimental proof of the existence of such correlation is one of the major goals of the present work.

- Systematic experimental information on formation and properties of the oxide films formed on nickel alloys and ferritic-martensitic steels under a representative set of water chemistry conditions was accumulated.

- One of the major practical results of the present work was the experimentally proven ability of the economical CDE technique to supply in situ data for ranking candidate structural materials for Generation-IV SCWR.

- A potential use of SRI's CDE arrangement for building in situ sensors for monitoring water chemistry in the heat transport circuit of Generation-IV SCWR was evaluated and proved to be feasible.

- Fracture surface topography analysis (FRASTA) analysis of the crack front movement indicates that cracks in austenitic stainless steels advance by formation of discontinuities ahead of the crack front, followed by their coalescence.

- Experimental data obtained during the reporting period allowed estimation of the crack growth rates for the first group of candidate structural materials - austenitic stainless steels. This group consisted of SS304 as a "baseline" material, with SS316 and SS347 as candidate materials. 


\section{INTRODUCTION}

\section{OVERVIEW}

This report describes the approach, methods used, and experimental and theoretical results for the work SRI International (SRI) has performed under NERI project 1-130, "Fundamental Understanding of Crack Growth in Structural Components of Generation IV Supercritical Light Water Reactors."

The Background subsection below highlights the place of this project in worldwide efforts to select in-vessel structural materials for supercritical water reactors (SCWRs). The importance of measuring oxide film properties for screening candidate alloys in situ, understanding fundamentals of material stability in supercritical water, and aiding in alloy design and selection is emphasized. An outline of SRI's approach is presented, along with an overview of the candidate structural materials.

The Purpose section outlines the goal and objective of the project work. The goal was to arrive at an understanding of the fundamentals of the degradation mechanism for the three groups of candidate structural materials - stainless steels, nickel alloys, and ferritic-martensitic steels - and to rank those materials according to their susceptibility to general and localized corrosion.

The Scope section contains detailed information on each task and subtask completed. Final testing of the experimental system and establishing of the experimental procedure for in situ measurements of the transport properties of the oxide films were completed at the beginning of the second year. The rest of the work proceeded in two parallel directions: (1) in situ measurements of the transport properties of the oxide films formed on stainless steels, nickel alloys, and ferritic-martensitic steels in supercritical water, and (2) stress corrosion cracking (SCC) tests of selected materials, followed by postexamination of the fracture surfaces to determine information about the crack propagation process.

The Methods, Assumptions, and Procedures section provides information about experimental techniques, current theoretical understanding of the transport processes in metal/oxide film/supercritical water systems, derivation of oxide film kinetic parameters from in

situ measurements, and correlation of the measured and derived kinetic parameters with susceptibility of the stainless steels to general and localized corrosion in supercritical water. 
This section also describes the experimental system, including the supercritical test reactors and water loop, the instrumentation for electrochemical measurements, and data acquisition and processing modules.

The Results and Discussion section contains the results obtained during the reporting period on the study of oxide films for candidate structural materials using in situ techniques based on the CDE approach. Postexamination of the composition, morphology, and thickness of the oxide films using scanning electron microscopy (SEM) and fracture surface analysis and estimation of crack growth rates with the fracture surface topography analysis (FRASTA) technique is also described.

The Conclusions and Future Work section summarizes the major accomplishments during the reporting period and outlines the envisioned future work.

\section{BACKGROUND}

The evaluation of corrosion and SCC behavior of alloys in supercritical water is a major emphasis in international efforts (Table 1) to select structural materials for SCWRs [1,2]. The process for selecting materials is envisioned to consist of (1) screening alloys to develop information on the broad response of the candidate alloys, (2) arriving at an understanding the basic phenomena and controlling mechanisms of corrosion and SCC in supercritical water, and (3) integrating theoretical modeling and the experimental approach to provide a rational basis for developing improved alloys [2].

Table 1. Organizations involved in SCWR projects worldwide

\begin{tabular}{|c|c|c|c|}
\hline Country/Region & National Laboratories & University & Industry \\
\hline United States & INEEL, ANL, ORNL & $\begin{array}{c}\text { Michigan, Wisconsin, MIT, } \\
\text { Notre Dame }\end{array}$ & $\begin{array}{l}\text { Westinghouse, } \\
\text { SRI International }{ }^{(*)}, \\
\text { Burns \& Roe }\end{array}$ \\
\hline Canada & - & - & $\mathrm{AECL}$ \\
\hline Japan & - & Tokyo Kyushu, Hokkaido & Toshiba, Hitachi, TEPCO \\
\hline Europe & $\begin{array}{l}\text { FZK (Germany), } \\
\text { CEA (France), } \\
\text { PSI (Switzerland), } \\
\text { VTT (Finland), } \\
\text { KFKI (Hungry) }\end{array}$ & $\begin{array}{c}\text { Manchester (U.K.), } \\
\text { University of Montenegro } \\
\text { (Montenegro) }\end{array}$ & $\begin{array}{c}\text { Framatome-ANP (France } \\
\text { and } \\
\text { Germany), } \\
\text { EdF (France), } \\
\text { Westinghouse Atom } \\
\text { (Sweden) }\end{array}$ \\
\hline Korea & KAERI & SNU, KAIST & - \\
\hline Russia & Institute, IPPE & & \\
\hline
\end{tabular}

. *Funded by U.S. DOE under the NERI program

This NERI project relied on a unique combination of advanced materials characterization techniques to contribute to the following efforts: 
- Screening Alloys. In situ characterization of the oxide films formed on candidate structural materials allows ranking materials according to their susceptibility to general and localized forms of environmentally assisted degradation, assuming that a correlation exists between oxide film properties and stability of materials. Conclusions from the oxide film studies are being compared with the direct results of parallel SCC experiments and fracture surface analysis to confirm correlations between oxide film properties and SCC, and to estimate crack initiation times and crack growth rates.

- Understanding basic phenomena. Kinetic parameters of the charge and mass transport processes in the metal/oxide film/supercritical water system are being derived from in situ oxide film studies to understand and quantify the rate-controlling processes and, eventually, the fundamentals of metal oxidation phenomena at supercritical temperatures.

- Providing a basis for the development of new alloys. In situ experimental information on oxide film resistance to charges in and mass transport of alloys of various composition is being integrated with current theoretical understanding of the relationship between properties of the oxide films and stability of materials. This knowledge is expected to aid in the material selection and development process by providing recommendations for the chemical compositions of the alloys depending on their expected locations in the reactor (or more specifically, depending on the local temperature and oxidizing conditions).

An overview of SRI's approach follows.

\section{In situ Methods to Study Environmentally Assisted Degradation of Materials}

The traditional method of studying corrosion phenomena has been to expose samples to the environment for a certain period and then analyze the samples with different ex situ techniques for typical surface markings produced by corrosion. This approach has its value, but to understand the fundamentals of corrosion phenomena it needs to be complemented by more fundamental information involving in situ investigation of the properties of the surface films (mostly oxides) being formed on the construction materials in contact with coolant.

The susceptibility of structural materials to different forms of corrosion is closely connected with the transport and reactions of chemical species on and in the oxide films forming on the materials' surfaces. Accordingly, to identify the rate-limiting steps for the corrosion 
processes, the transport of chemical species and related reactions on and in oxide films need to be measured and understood.

A possible approach for describing environmentally assisted degradation is to assume that the transport of species through the oxide films is one of the rate-controlling factors for general and localized corrosion. Assuming that the transport of ionic defects through the oxide film contributes to the degradation rate, a correlation should exist between the degradation rate and the oxide film properties. Generally, the more ionic defects an oxide film contains, and thus the less stable the film is, the faster the transport of ionic species through the film occurs. Our experimental methods allow us to identify the nature of various oxide defects and quantify their contributions to oxide breakdown.

Figure 1 illustrates the sequence of steps in the SRI approach $[3,4]$ to correlate structure and composition of the oxide films and performance of the alloys.

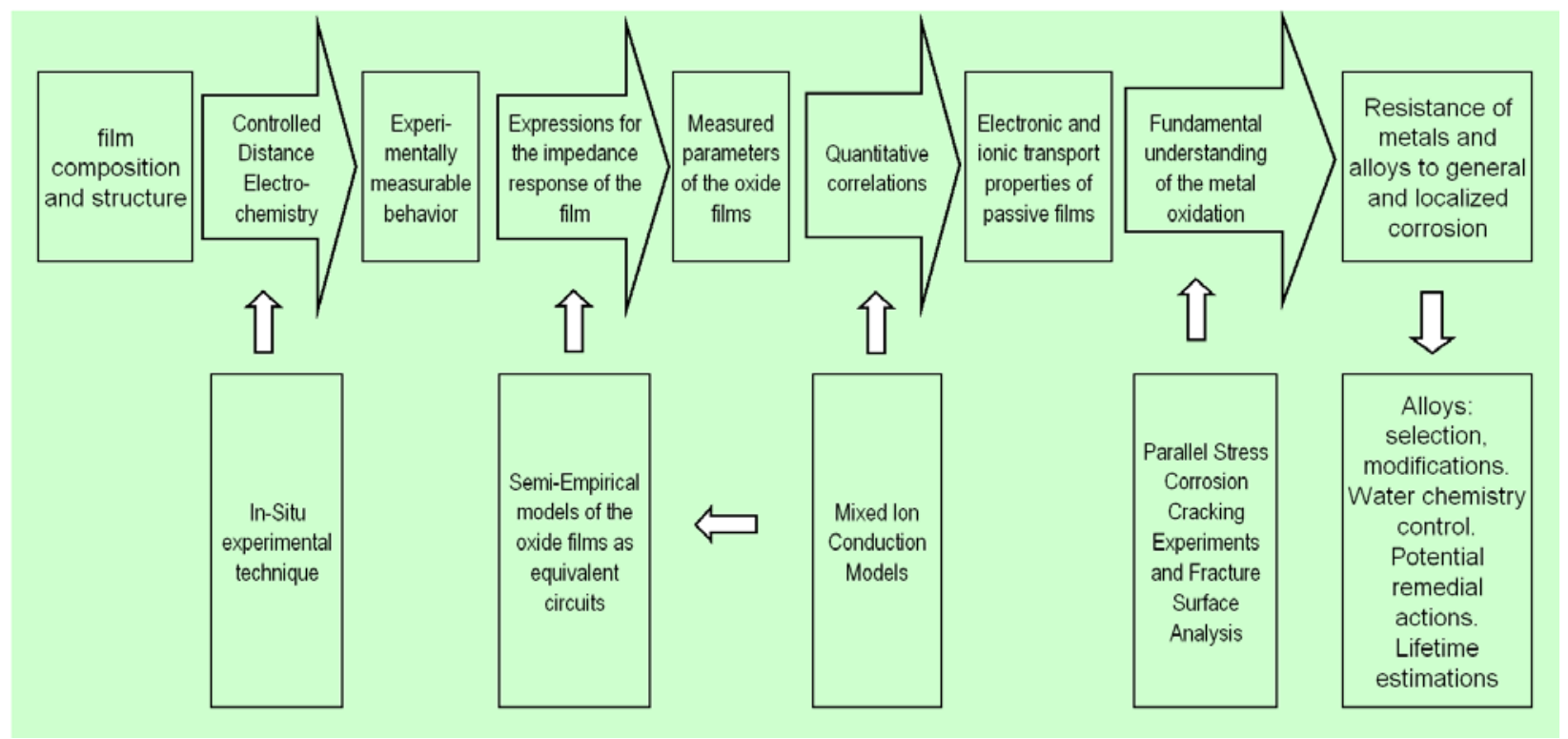

Figure 1. SRI's approach to studying the fundamentals of material stability in supercritical water.

The possibilities of performing reliable electrochemical experiments [e.g., in simulated boiling water reactor (BWR) coolants] have been limited because of the low conductivity of the medium. The problems related to the coolant's low conductivity can be overcome, however, by using the controlled distance electrochemistry (CDE) technique. This technique was originally introduced at VTT (Finland) for subcritical temperatures and later developed for supercritical temperatures under the present NERI project at SRI's Materials Research Laboratory. The SRI system can be used for unique materials reliability studies in high-temperature gas media and in aqueous environment at sub- and super-critical temperatures. 
The results of these measurements are being used in interpreting the rate-limiting processes in corrosion phenomena and as input data for modeling purposes. The main results are material-specific parameters describing the material's susceptibility to general and localized corrosion, SCC in particular, provided that the crack growth rate is determined by the transport of chemical species through the oxide films within a crack. The determination of these materialspecific parameters may lead to the identification of key remedial actions, alloy modifications, and selection of the protective coatings by which the susceptibility can be decreased.

In this project we employed a combination of the in situ oxide film characterization studies (various combinations of the CDE technique) with conventional SCC studies complemented by FRASTA, the SRI-developed fracture surface postexamination technique. FRASTA examination provides information on the evolution of crack nucleation and growth. We demonstrated that a comparison of the measured film transport parameters and actual crack growth rate can provide confirmation about the correlation of oxide film properties and the susceptibility of materials to general and localized corrosion at supercritical temperatures.

Detailed information on using CDE and FRASTA is presented in the Assumptions, Methods, and Procedures section.

\section{Candidate Materials for SCWR In-Vessel Components}

The set of candidate materials SRI has examined is in synchronization with other NERI SCWR projects and with international SCWR materials reliability programs. Requirements for the reliability of in-vessel structural materials in SCWRs are summarized in the recently published SCWR reference design [1]. The two most significant materials-related factors to be considered in transition from the current light water reactor (LWR) designs to the SCWR are (1) the increase in coolant temperature from $300^{\circ} \mathrm{C}$ to $400-500^{\circ} \mathrm{C}$, and (2) compatibility of the candidate materials with the currently unknown water chemistry in the SCWR heat transport circuit. Water chemistry is anticipated to have its major impact on the in-vessel structural components [2].

Current LWR experience suggests that the following environmentally assisted degradation mechanisms may be life-limiting factors for SCWR in-vessel components: intergranular stress corrosion cracking, irradiation-assisted stress corrosion cracking (IASCC), and corrosion fatigue. These mechanisms are know to be affected by several material and environmental factors: (1) the metallurgical structure, including the presence of $\mathrm{M}_{23} \mathrm{C}_{6}$ phases, phase morphology, and depletion of $\mathrm{Cr}$ in zones adjacent to grain boundaries; (2) irradiation effects on grain boundary impurity segregation; and (3) the chemistry of the aqueous environment, especially concentrations of the oxidizing and reducing species. 
As a result of experience in operating LWRs and supercritical fossil plants, four broad categories of alloys are being considered as candidates for reactor internal components: (1) austenitic stainless steels, (2) ferritic-martensitic steels, (3) high alloys ( $\mathrm{Fe}<50 \% \mathrm{wt}$.), and (4) Ni-based alloys. Traditional structural materials such as SS304 and SS316 (including L variants) should be included in experiments at supercritical temperatures as benchmark materials [2].

Knowledge about the behavior of candidate materials in supercritical water is insufficient to rank these alloy categories in terms of general corrosion and resistance to SCC and IASCC. It is well known that hardening, solute segregation, and embrittlement produced by displacement damage can accelerate intergranular SCC in normal water. In addition, the water in a reactor heat transport circuit becomes more oxidizing because of radiolysis, further aggravating SCC. Even more aggressive conditions may well occur in supercritical water under irradiation. 


\section{PURPOSE}

The purpose of this project work was to arrive at an understanding of the fundamentals of the stability of candidate structural materials in supercritical water and to rank candidate alloys according to their susceptibility to general and localized corrosion under anticipates conditions of the SCWR heat transport circuit.

To meet this goal, SRI's objectives were to:

- Development versatile and economical supercritical controlled-distance electrochemical techniques suitable for experimental determination of the role of charge and mass transport processes in metal/oxide film/supercritical water on crack growth in candidate structural materials.

- Build experimental system for parallel electrochemical and stress corrosion cracking studied in supercritical water under controlled water chemistry conditions.

- Measure the relationships between parameters of oxide films and degradation of stainless steels at supercritical temperatures.

- Rank, using advanced electrochemical techniques, the influence of ionic species on the oxide films forming on metal surfaces exposed both to simulated SCWR bulk coolant and to simulated crack chemistry conditions.

- Evaluate the role of electrochemical reactions and properties of oxide films in the crack nucleation and growth mechanism under supercritical conditions.

- Obtain information on crack nucleation times and crack growth rates by analyzing the conjugate fracture surfaces using SRI's FRASTA technology.

- Correlate electrochemical information on material-environment interactions with crack nucleation and growth data from FRASTA to delineate the fundamentals of uniform and localized degradation of the stainless steels in Generation-IV SCWR.

The first year's work was focused on building experimental system and design of controlled distance electrochemistry arrangement for supercritical water. The second and third year's work was focused on alloy screening to develop information on the broad response of the candidate groups of alloys. The second and third year's work has provided valuable knowledge about the basic phenomena in the metal/oxide film/supercritical water systems and evaluation of the role of the oxide films in the resistance of stainless steels to SCC in supercritical water. The anticipated practical value of this project work is the provision of a technical basis for developing improved compositions of alloys through the integration of theoretical considerations and experimental results obtained during the reported period. 


\section{SCOPE}

The project consisted of three tasks:

1. Development of a CDE technique for supercritical temperatures.

2. Electrochemical characterization of the oxide film properties of candidate materials in supercritical conditions, both in bulk and within cracks.

3. Experimental investigation of crack initiation and propagation, and estimation of the lifetime of candidate structural materials.

\section{TASK 1. DEVELOPMENT OF A CDE TECHNIQUE FOR SUPERCRITICAL TEMPERATURES}

The overall objective of Task 1 was to assess the suitability of electrochemical techniques to study corrosion phenomenon at supercritical conditions. A versatile and economical CDE arrangement suitable for experimental investigation of the roles of electrochemical reactions and properties of oxide films in the crack growth mechanism in supercritical conditions was designed during the first year.

The following Task 1 components were completed during the first year:

- Design and assembly of a supercritical water loop.

- Design of a CDE arrangement for supercritical temperatures.

- $\quad$ Test of CDE at supercritical temperatures.

The following components of Task 1 were completed during the second year.

- $\quad$ Long-term stability testing of the CDE arrangement at supercritical temperatures.

- $\quad$ Establishment of an experimental procedure for CDE measurements.

\section{TASK 2. AN ELECTROCHEMICAL CHARACTERIZATION OF THE OXIDE FILM PROPERTIES ON CANDIDATE MATERIALS IN SUPERCRITICAL CONDITIONS IN BULK AND WITHIN CRACKS}

The objective of Task 2 was to obtain experimental information on the protective properties of the oxide films for candidate structural materials under bulk and occluded (crack/crevice) chemistry conditions. 
The following components of Task 2 for a first group of candidate structural materialsaustenitic stainless steels - were completed during the second year and for nickel alloys and ferritic-martensitic steels during the third year:

- Characterization of the formation and reduction kinetics and the properties of metal oxide films by using the contact electric resistance (CER)

- Measurement of the solid contact impedance spectra of oxide films by using contact electric impedance (CEI).

- Characterization of the oxidation and reduction kinetics and mechanisms of metals as well as the properties of metal oxide films by using thin-layer electrochemical (TLEC) impedance measurements.

- CDE measurement under simulated crack conditions.

- Ranking of the influence of ionic species on the oxide films forming on metal surfaces exposed both to simulated SCWR bulk coolant and to simulated crack chemistry conditions, using advanced electrochemical techniques.

- Quantification and interpretation of the rate-limiting processes in the corrosion phenomena and the role of electrochemical reactions and properties of oxide films in the crack growth mechanism under supercritical conditions.

- Derivation of material-specific parameters that describe the susceptibility of metals to SCC in supercritical coolant conditions for the materials in which crack growth is controlled by the phenomena in oxide films within the crack.

- Identification of candidate remedial actions (changes in water chemistry, material chemical composition, and metallurgical parameters, in particular the degree of cold work) that can decrease susceptibility to SCC.

\section{TASK 3. EXPERIMENTAL INVESTIGATION OF CRACK INITIATION AND PROPAGATION AND ESTIMATION OF THE LIFETIME OF CANDIDATE STRUCTURAL MATERIALS}

The objectives of Task 3 were to (1) obtain information on crack nucleation times and crack growth rates via analysis of conjugate fracture surfaces by using SRI's FRASTA technology, and (2) correlate electrochemical and fracture mechanics (FM) experimental data to establish correlations between the properties of the oxide films and crack growth rates.

The following components of Task 3 for a first group of candidate structural materialsaustenitic stainless steels - were completed during the second year and for nickel alloys and ferritic-martensitic steels during the third year:

- Exposure of loaded specimens to a supercritical aqueous environment simultaneously with electrochemical studies of the oxide films on the same materials.

- Examination of the fracture surfaces of specimens by using the FRASTA technique. 
- Identification of crack nucleation sites and times for specimens made of different candidate materials.

- Use of FRASTA to determine crack front formation and movement, including formation of discontinuities ahead of the crack and their possible coalescence later while the crack is advancing.

- Estimation of crack growth rates for different candidate materials under a set of conditions using FRASTA for examination of conjugate fracture surfaces. In addition to autoclave experiments under constant chemistry conditions, several experiments used "time marks" on tested specimens: these time marks were created by increasing/decreasing the injection of oxygen to increase/decrease crack growth rates.

- Correlation of electrochemical information on material-environment interactions with crack nucleation and growth data from FRASTA to delineate the fundamentals of uniform and localized degradation of structural materials in Generation-IV SCWR and estimate the life-times of candidate materials for structural components under a variety of normal and offset operating conditions. 


\section{METHODS, ASSUMPTIONS, AND PROCEDURES}

As outlined in the Scope section, the work proceeded in two parallel efforts:

1. Electrochemical characterization of the oxide film properties on candidate materials in supercritical conditions.

2. Experimental investigation of crack initiation and propagation for candidate structural materials.

An overview of the methods, assumptions, and procedures is given below, followed by an expanded description of each item.

Electrochemical characterization of the oxide film properties involved impedance measurements using three modifications of the CDE technique: Contact Electric Resistance (CER), Contact Electro-Chemical Impedance (CEI), and Thin-Layer Electrochemistry (TLEC). The CER technique provides information on the electronic transport properties of the oxide films. The CEI technique provides information on the impedance of the metal/oxide/metal system. The TLEC technique provides information on the impedance of the metal/oxide film/supercritical water system.

Both metal/film/metal (or the "symmetrical configuration") and metal/oxide film/supercritical water (or the "asymmetrical configuration") are represented by equivalent circuits to allow in-depth analysis of the charge and mass transfer processes in symmetrical and asymmetrical configurations. These equivalent circuits are based on current theoretical models of the oxide films' behavior. Some elements of the equivalent circuits have an electrical analog (e.g., charge transfer resistance), and some elements represent transport phenomena, which cannot be formalized as a simple electrical analog (e.g., Young's impedance and Warburg diffusion). A wide variety of the "extended elements" describing a variety of the transport processes occurring in compact and porous oxides in contact with a metal and an electrolyte may be included in the model, if necessary.

Symmetrical configuration metal/film/metal is expected to establish the dominant role of the electronic carriers, as opposed to the dominant role of the ionic carriers in the asymmetrical configuration. Accordingly, contributions of the electronic and ionic carriers can be separated by using both CEI and TLEC measurements for the same metal/film/electrolyte system. 
Once impedance data are fitted into a "physical-model-based" electrical circuit, fundamental kinetic parameters such as diffusion coefficients can be determined from the impedance measurements. This step is based on the assumption that the existing theoretical models of the oxide films capture the essence of the transport phenomena inside the film and on film interfaces with metal and solution. Comparison of the derived kinetic parameters for various alloys and environmental conditions allows understanding the fundamentals of materials degradation in supercritical water and also serves to rank candidate materials according to their susceptibility to general and localized forms of corrosion.

A quantitative concept of the "oxide film stability" was introduced, with film stability defined as resistance to charge and mass transfer processes. Use of the experimentally obtained film stability allowed ranking of candidate structural materials with respect to their susceptibility to environmentally assisted degradation in supercritical water. Information on film stability itself was not sufficient to estimate the lifetime of candidate materials under anticipated operating conditions.

In situ studies of the oxide films have been complemented by Fracture Mechanics (FM) testing and examination of the fracture surface in order to estimate crack nucleation time and crack growth rates, which constituted a parallel effort under this NERI project. That effort includes direct studies of crack growth in preloaded and fatigue precracked FM specimens exposed to supercritical water. A special type of the FM specimens was designed during the first year of the project [3]. FM and CDE specimens were exposed to the identical supercritical water environment in two reactors [3]. FM specimens were extracted from the reactor after long-term exposure for examination using the FRASTA technique of the crack evolution process. In addition to crack growth rates, more fundamental information on crack formation and movement process was obtained from FRASTA analysis.

The final step in our approach was to compare kinetic parameters of the oxide films derived from CDE measurements and data of crack propagation derived from FRASTA analysis of the conjugate fracture surfaces. This comparison, as expected, revealed existence of correlation of the oxide film stability (or film resistance to transport processes) and crack growth in candidate structural materials. Eventually, material parameters (e.g., chemical composition and ability to form protective oxide films) controlling degradation rate may be identified and candidate materials may be ranked according to their stability in supercritical water.

An expanded description of the methods and procedures is given below. 


\section{IN SITU MEASUREMENTS OF THE KINETICS OF CHARGE AND MASS TRANSFER IN OXIDE FILMS}

This section introduces the concepts of the symmetrical and asymmetrical configurations for in situ measurements of the charge and mass transfer across the oxide films formed on structural materials in supercritical water. Each partial process was represented by an element of the "physical-based" equivalent electrical circuit of a system under investigation. Relationships between parameters of the "electric circuit elements" and physical parameters of the oxide films were established to derive film transport properties from the impedance measurements.

\section{Role of Controlled Distance Electrochemistry in Materials Reliability Assessment}

The traditional way of studying corrosion phenomena has been to expose samples to the environment for a certain period and then analyze the samples with different ex situ techniques for typical surface markings produced by corrosion. This approach has its value, but to understand fundamentals of corrosion phenomena this approach needs to be complemented by a more fundamental approach involving in situ investigation of the properties of the surface films (mostly oxides) that are being formed on the construction materials in contact with coolant. The susceptibility of structural materials to different forms of corrosion is closely connected with the transport and reactions of chemical species on and in the oxide films forming on material surfaces. Accordingly, to identify the rate-limiting steps for the corrosion processes, the transport of chemical species and related reactions on and in oxide films need to be measured and understood. The main focus of this project was to extend earlier approaches developed for metal oxide films under existing LWR conditions to supercritical conditions corresponding closely to those presumed to exist during different operational stages of supercritical power plants.

The experimental parameters needed for modeling the corrosion process are measured mainly with in situ electrochemical techniques. The possibilities of performing reliable electrochemical experiments, for instance, in simulated boiling water reactor (BWR) coolants, have been limited because of the low conductivity of the medium. The problems related to the low conductivity of the coolant can be overcome by means of the CDE arrangement (Figure 3), which was recently developed at VTT Manufacturing Technology, Finland. This technique can be applied to perform contact electric resistance (CER), electrochemical impedance spectroscopic (EIS), and contact electric impedance (CEI) measurements in high-temperature aqueous environments [5-6].

Figure 2 provides a schematic principle of the CER and CEI techniques. Two specimen surfaces are brought into contact and disconnected at regular intervals using a chosen frequency. 
When the surfaces are disconnected, the surfaces are exposed to the environment, and oxidation or any other process resulting in changes of the surface properties may take place. When the surfaces are in contact, an AC impedance spectrum is acquired (CEI measurements) or DC resistance is measured (CER technique). The low frequency limit of the contact electric impedance spectrum is practically identical to the value measured by the DC resistance CER technique. The measured CEI spectrum contains information on the kinetics of the transport processes occurring in the film at any given moment.

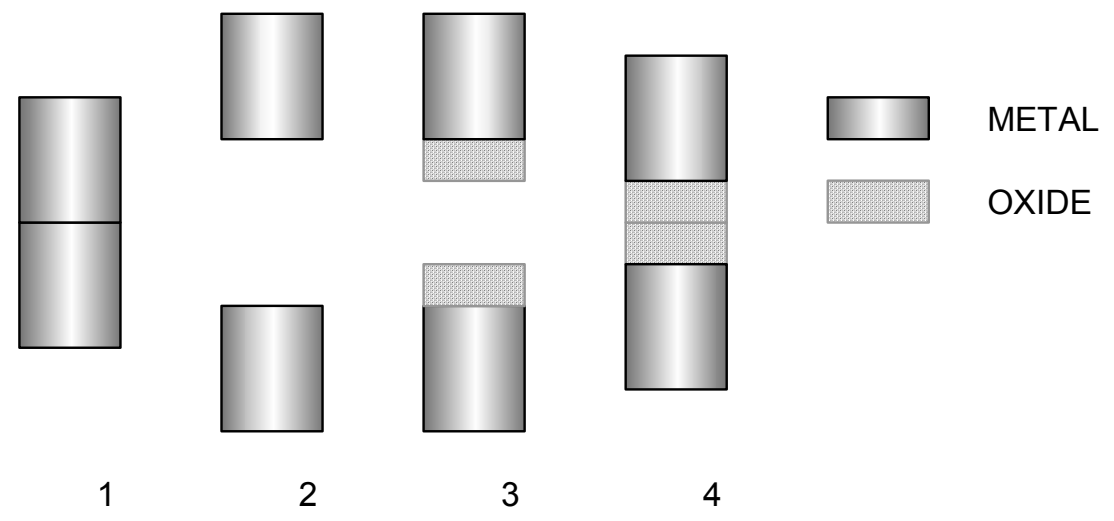

Figure 2. Controlled Distance Electrochemistry measurements: (1) specimens are in contact for CER and CEI measurements, (2) specimens are exposed to the environment, (3) oxidation takes place, (4) specimens are in contact for CER and CEI measurements.

The CDE experimental setup is shown schematically in Figure 3 was used in this project for the following types of measurements:

- Thin-layer electrochemistry (TLEC): Impedance measurements to characterize the oxidation and reduction kinetics and mechanisms of metals as well as the properties of metal oxide films in simulated supercritical coolant environments.

- Contact electric resistance (CER): Solid contact measurements to investigate and/or monitor the electronic properties of surface films.

- Contact electric impedance (CEI): Measurements to determine the solid contact impedance spectra of oxide films.

The results from these measurements were used in interpreting the rate-limiting processes in corrosion phenomena and as input data for modeling purposes. The main results were material-specific parameters describing the material's susceptibility to stress corrosion cracking in supercritical coolant conditions, provided that the crack growth rate is determined by the transport of chemical species through the oxide films within a crack. The determination of these material-specific parameters may lead to the identification of key remedial actions by which the susceptibility can be decreased. 

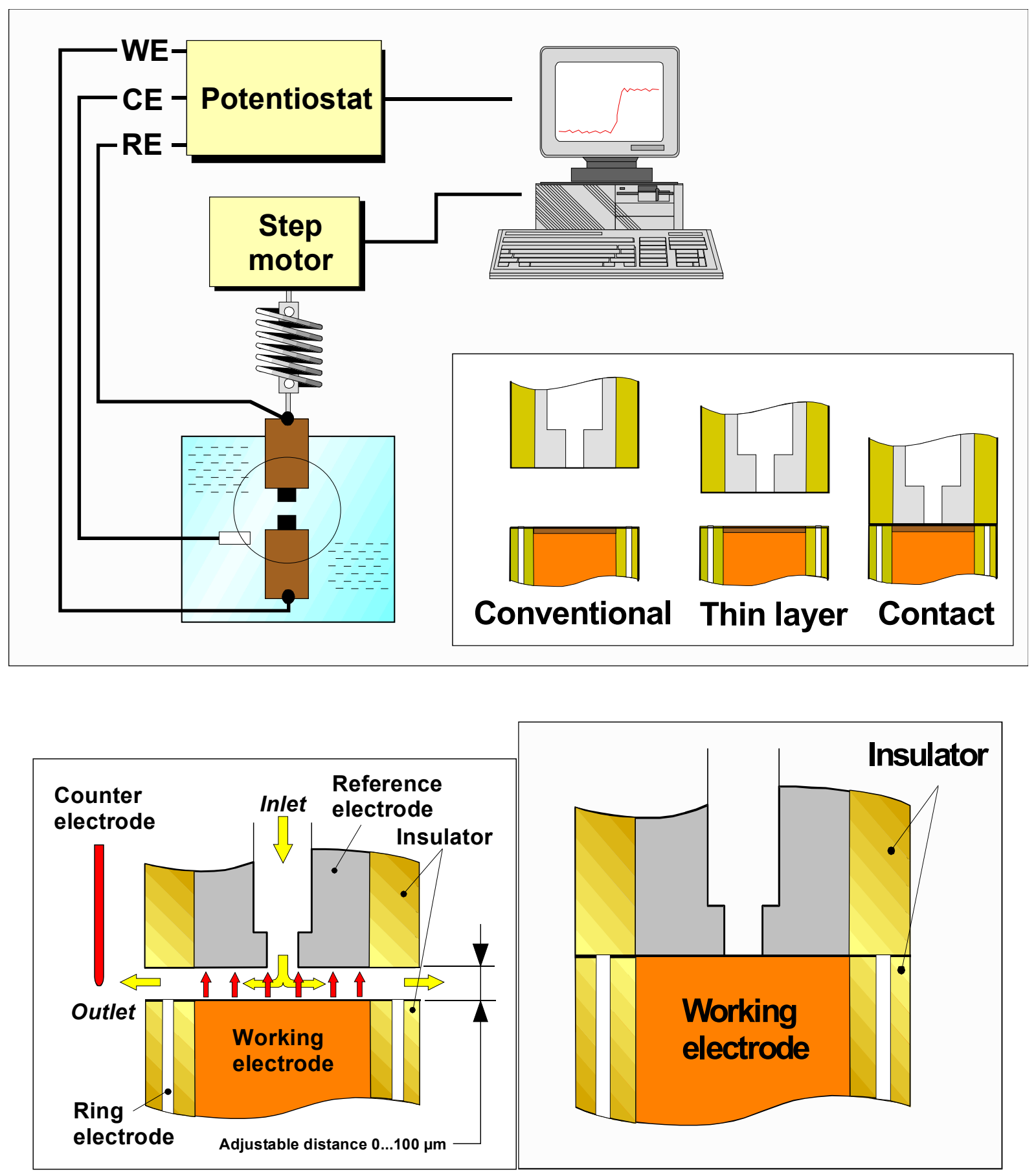

Figure 3. A scheme of the controlled-distance electrochemistry (CDE) arrangement and its different applications.

A possible approach in the description of environmentally assisted degradation is to assume that the transport of species through the oxide films on the crack walls is one of the ratecontrolling factors for crack growth. This may, for instance, be the case if the crack propagates 
via the accumulation of vacancies in the metal substrate. These vacancies $\left(\mathrm{V}_{\mathrm{m}}\right)$ can be generated at the metal/film interface as follows:

$$
\mathrm{m}+\mathrm{V}_{\mathrm{M}}^{3-} \rightarrow \mathrm{M}_{\mathrm{M}}^{\mathrm{III}}+3 \mathrm{e}^{-}+\mathrm{V}_{\mathrm{m}}
$$

In other words, a cation vacancy reacts with a metal atom, resulting in the formation of a cation in the oxide film and a metal vacancy in the metal substrate. For this reaction to occur, cation vacancies generated at the film/solution interface have to be transported to the metal/film interface.

Assuming that the transport of ionic defects through the oxide film on the crack walls contributes to the crack growth rate, a correlation should exist between the crack growth rate and the oxide film properties. Generally, the more ionic defects an oxide film contains, and thus the less stable the film is, the faster the transport of ionic species through the film.

The present work will endeavor not only to characterize the influence of different ionic contents in the coolant on the transport rate of ionic defects in oxide films forming on candidate structural materials in supercritical water, but also to correlate these results with crack growth data obtained in separate experiments.

In our previous work [6] we applied the CDE arrangement described above to estimate the impact of sulfate ions on the cracking behavior of stainless steels and nickel base alloys (AISI 316, Inconel 82 and 182) in ultrapure deoxygenated water at $288^{\circ} \mathrm{C}$. In that study, electrochemical impedance spectra were measured for three different materials at all the sulfate levels after an exposure of about $24 \mathrm{~h}$ to simulated crack conditions. For example, the spectra obtained for the materials in the coolant with $\cong 0$ and $10,000 \mathrm{ppb} \mathrm{SO}_{4}{ }^{2-}$ ions are shown in Figure 4.

Qualitatively, the spectra for all the materials at all the sulfate levels exhibit a lowfrequency linear part that transforms into a semicircle at very low frequencies. In addition, a small high-frequency impedance loop can be observed in several cases. These types of spectra are typical for a situation in which both a charge transfer reaction (e.g., oxidation of metal) and a transport process under a concentration gradient contribute to the behavior. Accordingly, the low-frequency part can most likely be attributed to a transport process in the film, while the high-frequency impedance loop is probably due to the charge transfer of the corrosion reaction or the cathodic reaction coupled with it. 

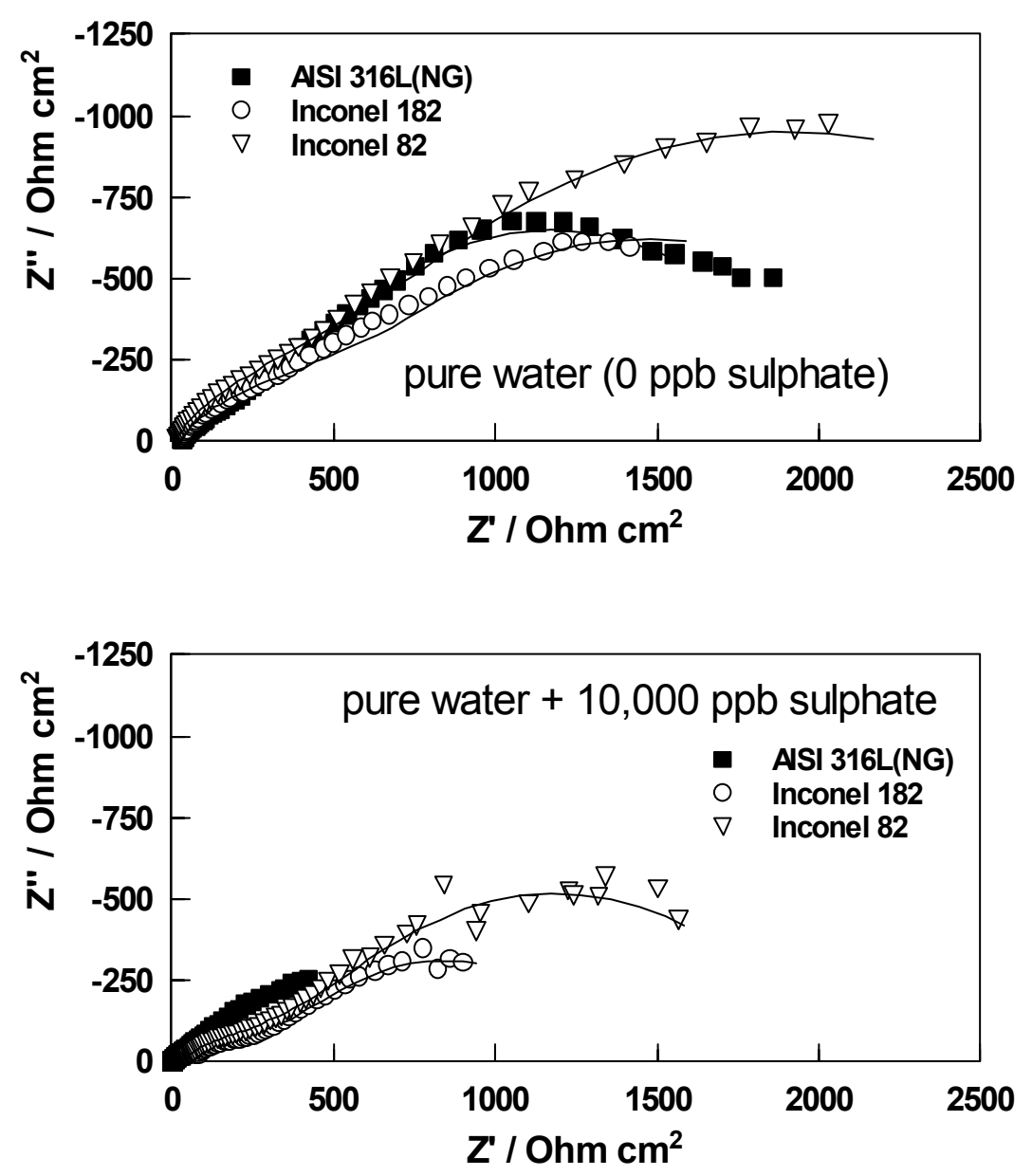

Figure 4. Electrochemical impedance spectra of AISI 316 (NG) stainless steel, Inconel Alloy 182, and Inconel Alloy 82 in the presence of 0 (top) and $10000 \mathrm{ppb}$ (bottom) sulfate ions in simulated crack conditions at $273^{\circ} \mathrm{C}$ (symbols: experimental points; lines: fitted results).

The impedance, i.e., the electrical transfer function, of an electrochemical system can be described by means of either an equivalent circuit or an analytical expression. The results obtained in the work described above were fitted using the following transfer function, which takes into account the features discussed above:

$$
\mathrm{Z}=\mathrm{R}_{\mathrm{el}}+\left\{\mathrm{j} \omega \mathrm{C}_{\mathrm{hf}}+\left[\mathrm{R}_{\mathrm{hf}}+\mathrm{R}_{\mathrm{D}} \tanh \left(\mathrm{j} \omega \tau_{\mathrm{D}}\right)^{\mathrm{n}_{\mathrm{D}}} /\left(\mathrm{j} \omega \tau_{\mathrm{D}}\right)^{{ }^{\mathrm{n}} \mathrm{D}}\right]^{-1}\right\}^{-1}
$$

where

$\mathrm{Z}=$ impedance of the system

$\mathrm{j} \quad=$ imaginary unit

$\omega=$ angular frequency of the A.C. signal

$\mathrm{R}_{\mathrm{el}}=$ electronic resistance of the electrolyte solution 
$\mathrm{R}_{\mathrm{hf}}=$ resistance corresponding to the high-frequency loop

$\mathrm{R}_{\mathrm{D}}=$ resistance corresponding to the low-frequency loop (i.e., the transport resistance)

$\mathrm{C}_{\mathrm{hf}}=$ capacitance corresponding to the high-frequency loop

$\tau_{\mathrm{D}}=$ time constant for the transport process

$\mathrm{n}_{\mathrm{D}}=$ exponent of the transport impedance function $\left(\mathrm{n}_{\mathrm{D}}=0.5\right.$ for linear diffusion toward a planar surface, 0.25 for linear diffusion in cylindrical pores).

The relative magnitudes of $\mathrm{R}_{\mathrm{hf}}$ and $\mathrm{R}_{\mathrm{D}}$ determine whether charge transfer or the transport of ions or ionic defects controls the overall rate of metal oxidation. If $R_{h f}>>R_{D}$, charge transfer controls the oxidation rate, whereas if $\mathrm{R}_{\mathrm{hf}}<<\mathrm{R}_{\mathrm{D}}$, transport of ions or ionic defects controls the oxidation rate. As indicated above, the rate of transport of ions or ionic defects in the film can be interpreted as a measure of the stability of the film: the lower the transport rate, the more stable the film.

The values obtained for the resistances $R_{h f}$ and $R_{D}$ as a result of the fitting of the impedance spectra showed that in all cases the transport of ions or ionic defects controls the overall rate of metal oxidation. Figure 5 shows the values of $\mathrm{R}_{\mathrm{D}}$ for the three materials as a function of sulfate content.

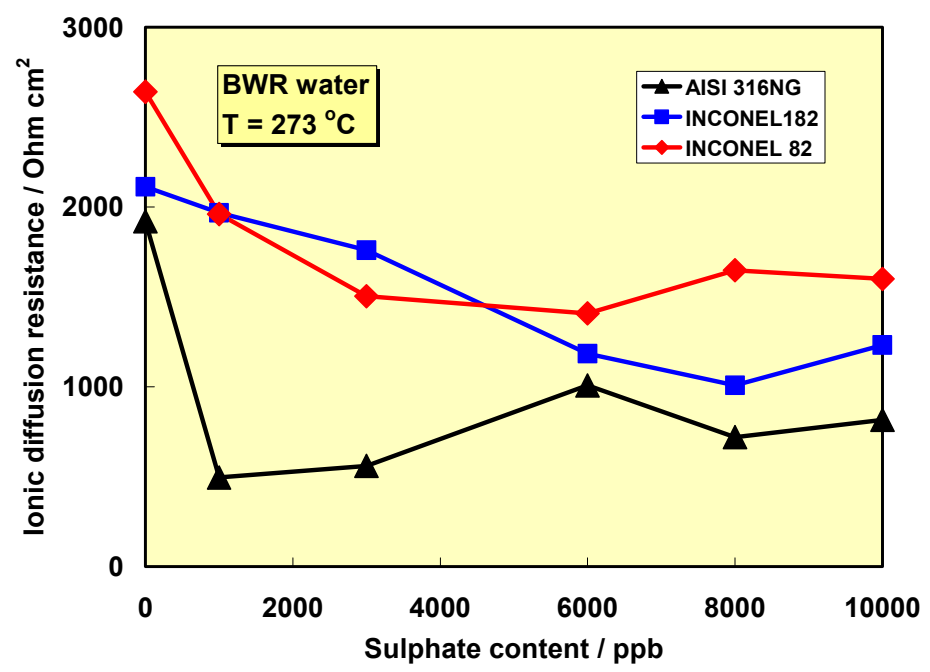

Figure 5. The value of the resistance $R_{D}$ for the transport of ions or ionic defects through the oxide film on AISI 316NG and Inconel alloys 182 and 82 as a function of sulfate ion content in simulated crack chemistry conditions.

The results given in Figure 5 show that the resistance of ionic diffusion, $R_{D}$ is generally highest in oxide films formed on Inconel alloy 82, while the lowest values are obtained for AISI 316 NG. For both Inconel alloy 182 and 82 , the value of $R_{D}$ decreases with increasing sulfate 
content at low sulfate levels and stabilizes at higher levels. The general trend for AISI $316 \mathrm{NG}$ is also a decrease in $\mathrm{R}_{\mathrm{D}}$ with increasing sulfate content, but the curve exhibits a minimum at $1000 \mathrm{ppb}$.

These results can be interpreted as showing that the oxide films are the most stable on Inconel alloy 82, the second most stable on Inconel alloy 182, and the least stable on AISI $316 \mathrm{NG}$. The stability of the films on all the materials decreases with increasing sulfate content.

A comparison of these results with empirical crack growth data results indicates a correlation between the stability of the oxide film on the crack walls and stress corrosion cracking susceptibility. A stress corrosion crack growth test series conducted at VTT on the same materials showed that Inconel alloy 82 is the least susceptible, as no stress corrosion cracking was observed at any of the applied bulk water sulfate levels ( 0, 10, 30 and $100 \mathrm{ppb})$. However, the tests showed that Inconel alloy 182 and AISI $316 \mathrm{NG}$ were susceptible at all of these sulfate levels. Also, the crack propagation rates in Inconel alloy 182 and AISI $316 \mathrm{NG}$ were dependent on the bulk water sulfate content, being higher at higher sulfate levels. More detailed results of these tests conducted in simulated BWR environment with $300 \mathrm{ppb}$ dissolved oxygen at $273^{\circ} \mathrm{C}$ were observed [7]. The dependence of crack propagation rates on coolant water sulfate ion level has been reported by other authors as well $[8,9]$.

It thus appears to be possible to determine in relatively short experiments a measurable quantity (e.g., $R_{D}$ ) that describes the transport of ions or ionic defects in the oxide film and thus the stability of the film. This quantity can be correlated at least qualitatively with the susceptibility to cracking. However, not only the sulfate content but also the material condition (e.g., the degree of cold work) has been found to have an impact on actual crack growth rates $[20,23]$. Thus, the oxide film properties alone cannot be regarded as determining crack propagation rates.

\section{Symmetrical and Asymmetrical Configurations for In situ Study of Oxide Films}

Electrochemical impedance spectroscopy has been widely applied to the study of the kinetics of the growth and dissolution of oxide films for a range of technically important metals and alloys [11-16]. The first step in this approach is to correlate measurement data with electrochemical models. Modeling the transfer function of an electrochemical system by means of an equivalent circuit is a common way to learn about its physics. Kinetic and thermodynamic properties determine the parameters and their values, whereas electrochemical mechanisms and topologies are mainly reflected in the connection scheme. The impedance elements in use provide information about, for example, the thickness of a layer, charge carrier concentration, reaction rate, and conductivity — among other characteristics of the oxide film examined. 
It has been demonstrated that, from a series of impedance spectra obtained at different experimental conditions (e.g., electrode potentials, temperatures, electrolyte concentrations), information can be obtained on the charge transfer processes at the metal/oxide or oxide/electrolyte interfaces and on the transport of ions and ionic defects through the oxide [310]. Information on the electrical properties of the film bulk and the interfacial regions can also be extracted [3,4,11-16]. However, the relatively high number of adjustable parameters often precludes unambiguous interpretation of impedance spectra in terms of a unique kinetic model.

Significant progress in overcoming this inherent difficulty has been achieved by introducing the concept of multiple transfer function analysis [17]. Within the framework of this concept, extensions of techniques such as the rotating ring-disc electrode and the electrochemical quartz microbalance to the frequency domain have found increasing acceptance in the scientific community [18-21]. Yet another technique based on the complex capacitance response has been recently put forward and applied to the study of metal dissolution and passivation processes [22].

An alternative that has been extensively developed is introducing impedance measurements in different configurations to determine the respective contributions of the bulk transport and interfacial transfer processes in the overall impedance response of thin films [2325]. This approach was extended for supercritical temperatures in the present NERI project $[3,4]$.

We used a combination of CDE measurements in:

- Asymmetrical configuration (metal/film/electrolyte).

- Symmetrical configuration (metal/film/metal).

These measurements, which were performed under a set of identical conditions, led to a significant decrease in the number of fitting parameters needed to reproduce an individual impedance spectrum. Thus, an increase in the statistical significance of parameter values extracted from impedance measurements was achieved [23-25].

The metal/film/metal symmetrical measurement configuration has frequently been used in the field of solid-state ionic and mixed conductors [26-28], as well as in investigating the high-temperature oxidation of metals and alloys [29-32]. Such a configuration has been applied for the study of wide variety of thin films [33-35]. Recently, a combination of impedance measurements in an asymmetrical and a symmetrical configuration has been employed to characterize the conduction mechanism of the passive film on pure $\mathrm{Fe}$ [36], $\mathrm{Fe}-\mathrm{Cr}$ and $\mathrm{Fe}-\mathrm{Cr}-\mathrm{Mo}$ alloys [37] in a borate solution at room temperature. The CEI measurements in the symmetrical configuration are an extension of the CER technique [38,39] to the frequency domain [33, 36, 37]. TLEC is used to measure the asymmetric configuration. 


\section{Thin Layer Electrochemistry}

A typical impedance spectrum of an oxide film measured during this project in asymmetric configuration in shown in Figure 6. At a first approximation, the high-frequency time constant in the spectra can be ascribed to a relaxation in a space charge layer or an interfacial charge transfer process, whereas the low-frequency part is best described as a Warburg-type transport impedance [40].

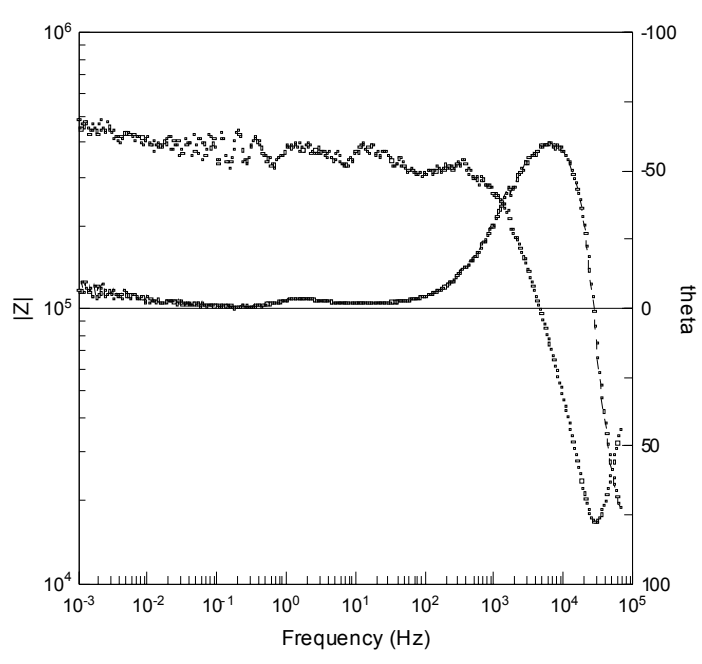

(a)

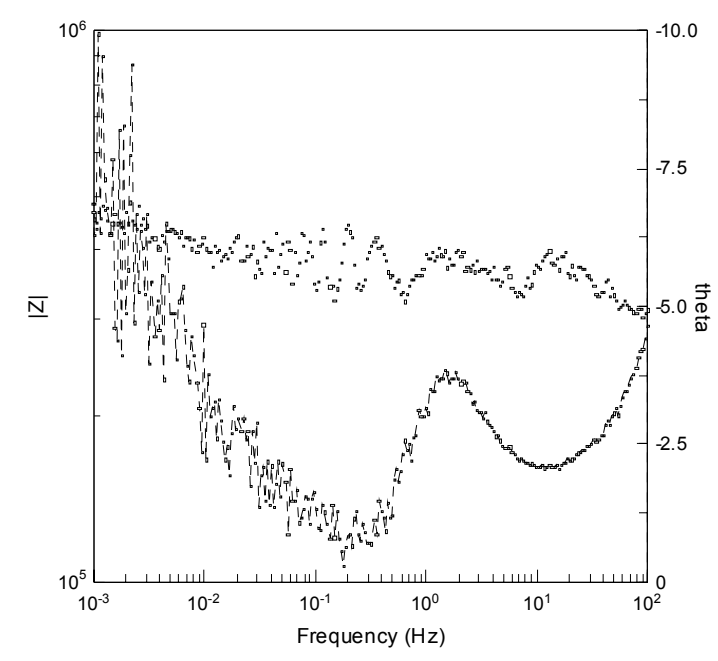

(b)

Figure 6. A typical TLEC impedance spectrum of the oxide film formed in supercritical water: (a) all frequencies, (b) low frequency part

By deconvolution of the spectra, it has been found that the high-frequency time constant is best described with Young-type impedance [35]

$$
Z_{Y}=\frac{p}{j \omega C} \ln \frac{1+j \omega \tau e^{\frac{1}{p}}}{1+j \omega \tau}
$$

Young impedance describes an insulating or semiconducting layer in which a steadystate profile of defects stretches from an interface to the bulk and gives rise to an exponential conductivity profile [41]

$$
\sigma=\sigma_{d} e^{-\frac{x}{x_{0}}}
$$

Young impedance is characterized by three parameters:

C - The capacitance of the insulating film or of the space-charge in the semiconductor

$\tau \quad$ - The time constant, $\tau=\frac{\varepsilon \varepsilon_{0}}{\sigma_{d}}$ 
$p \quad$ - The relative penetration depth, $p=\frac{x_{0}}{L}$

$L \quad$ - The film thickness

$\varepsilon \quad$ - The dielectric constant of the oxide film

$\varepsilon_{0}$ - The dielectric permittivity of vacuum

$\sigma_{d}$ - The defect-induced interfacial conductivity.

Young impedance can be approximated by a constant phase element (CPE) in a frequency range [42] where

$$
\frac{1}{p}>>\omega \tau
$$

The low-frequency part of the impedance is approximated as a generalized finite-length Warburg impedance with a conductive boundary in series with a charge transfer or migration resistance $R_{c t}$ :

$$
Z_{F, A}=R_{c t}+R_{W, A} \frac{\tanh \left(j \omega \tau_{W, A}\right)^{\beta}}{\left(j \omega \tau_{W, A}\right)^{\beta}}
$$

where

$\mathrm{RW}, \mathrm{A} \quad$ - transport resistance in asymmetrical configuration

$\tau_{W, A} \quad$ - time constant of the transport process in the asymmetrical configuration

$\beta \quad$ - exponent; for linear diffusion towards a planar surface $\beta=0.5$.

The total impedance is then calculated as follows

$$
Z_{A}=R_{e}+\frac{1}{\frac{1}{Z_{Y}}+\frac{1}{Z_{F, A}}}
$$

where $\mathrm{R}_{e}$ is the uncompensated resistance of the electrolyte. The impedance functions $Z_{\mathrm{Y}}$ and $Z_{\mathrm{F}, \mathrm{A}}$ are connected in parallel (see Figure 7) because the function $\mathrm{Z}_{\mathrm{Y}}$ can be regarded as the nonideal capacitance of a semiconductor layer with spatially and energetically variable donor or acceptor densities [43], whereas $Z_{\mathrm{F}, \mathrm{A}}$ accounts for the Faradaic impedance of generation, transport, and annihilation of ionic point defects [43]. 


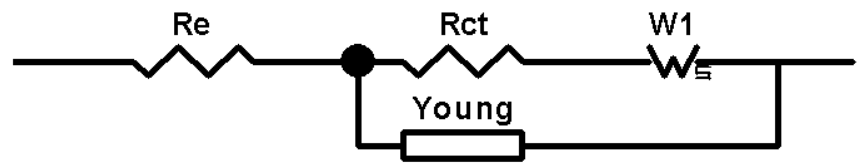

Figure 7. Equivalent electrical circuit for metal/film/electrolyte (asymmetrical) configuration.

\section{Contact Electric Impedance}

Contact electric impedance measurements are common in the field of solid-state ionics [26-32]. Symmetrical configuration metal/film/metal is expected to establish a predominant role of the electronic carriers as opposed to the ionic carriers in the asymmetrical configuration. Accordingly, contributions of the electronic and ionic carriers can be separated by using both CEI and TLEC measurements for the same metal/film/electrolyte system. Figure 8 shows the typical contact impedance spectrum of the oxide film measured in the present work.

The high-frequency part of the spectra in both symmetrical and asymmetrical configurations is attributed to the electronic properties of the space-charge layer in the oxide. The low-frequency spectra in the symmetrical configuration also make up a low-frequency time constant at $1-10 \mathrm{~Hz}$, which is attributed to a finite length diffusion process.

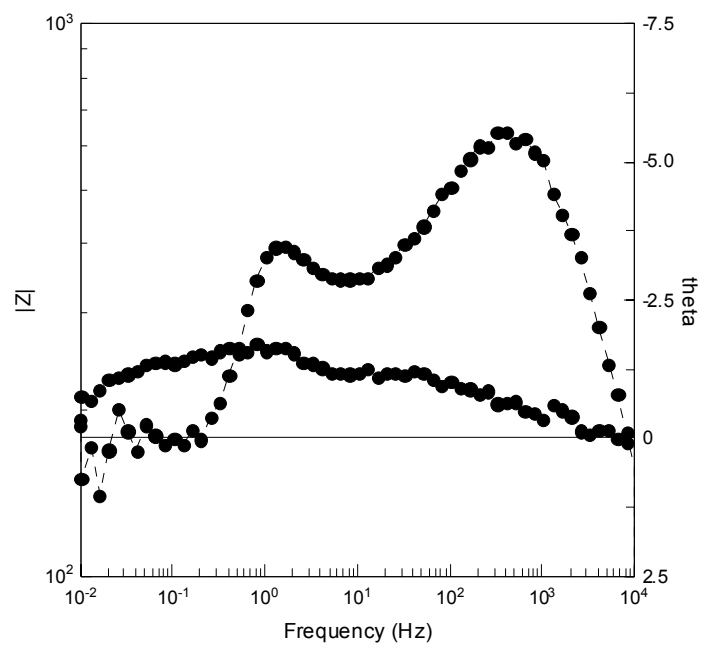

Figure 8. Typical CEI impedance spectrum of the oxide film formed in supercritical water.

In terms of the equivalent circuit elements, the metal/film/metal configuration was represented by finite-length diffusion impedance in series with a kinetic or migration resistance, analogous to the asymmetrical case [compare Eq. (4)]: 


$$
Z_{F, S}=R_{m}+R_{W, S} \frac{\tanh \left(j \omega \tau_{W, S}\right)^{\beta}}{\left(j \omega \tau_{W, S}\right)^{\beta}}
$$

where

Rw,S - transport resistance in symmetrical configuration

$\tau_{W, S} \quad$ - time constant of the transport process in the symmetrical configuration.

The characteristic frequency range of time constant $\tau_{W, S}$ for supercritical temperatures is about 2 orders of magnitude lower than that for $\tau_{W, S}$ measured for subcritical temperatures. At the same time, the comparison of previous measurements at $200^{\circ} \mathrm{C}$ and $300^{\circ} \mathrm{C}$ clearly indicates the tendency of the low-frequency time constant $\tau_{W, S}$ to shift toward lower frequencies with increasing temperature. Comparison of time constants for symmetrical $\left(\tau_{W, S}\right)$ and asymmetrical $\left(\tau_{W, A}\right)$ configurations provides a valuable insight about the type of transport processes in the oxide film. In the case of the previous measurements for subcritical temperatures, $\tau_{W, S}$ was observed for frequencies 1 or 2 orders of magnitude higher than $\tau_{W, A}[24,33,35,37]$. Accordingly, the transport process associated with time constant $\tau_{W, S}$ is related to current carriers that attain their steady state transport rate faster than those associated with $\tau_{W, A}$ in the asymmetrical configuration. Thus, measurements at subcritical temperatures in the asymmetrical configuration the ionic point defects were the unblocked current carriers, whereas in the symmetrical configuration this role was played by the electronic defects. In addition, the values of the impedance at low frequencies were about 1 to 2 orders of magnitude lower in the symmetrical configuration than in the asymmetrical one, indicating that the steady state conductivity of the system is higher in the symmetrical configuration. This finding also points to a predominant role for the electronic carriers in the symmetrical configuration and the ionic carriers in the asymmetrical configuration at subcritical temperatures.

Observed temperature shift in time constants at supercritical temperatures indicates the possibility of a more complex relationship between charge carriers in mixed-conducting films at supercritical temperatures. The ability of different models to interpret the results quantitatively and a tentative physical picture of the conduction mechanism are presented in the Results and Discussion section.

\section{Kinetic Models for the Asymmetrical Configuration}

One of the kinetic models for the growth and conduction mechanism of continuous oxide films on metals and alloys is the point defect model (PDM) [14, 44-46]. PDM describes the metal/oxide/electrolyte system at steady state in terms of generation, transport, and consumption of point defects (anion and cation vacancies and interstitial cations) under the influence of both 
the concentration and potential gradients in the oxide and at its boundaries with the metal and the electrolyte [44-46]. Both the steady state current and impedance response of a metal covered with a passive film in contact with an electrolyte have been derived and successfully compared to experimental data [44-47]. In recent years, the model was further developed at VTT [36, 43, $48,49]$ and by others [50, 51]. The main addition proposed by VTT in terms of the mixedconduction model (MCM) was the derivation of the Young impedance (Eq. 3) in terms of the steady state concentration gradients of vacancies and interstitial ions in the oxide [43, 48, 49].

For the sake of simplicity, it was assumed that the oxide film is a mixed conductor with a single ionic and a single electronic current carrier and that the ionic carrier is generated at the metal/film (M/F) and consumed at the film/solution $(\mathrm{F} / \mathrm{S})$ interface

$\mathrm{M} / \mathrm{F}$ interface

$$
\begin{gathered}
m \rightarrow M_{i}^{\bullet \bullet}+V_{m}+2 e^{\prime} \\
m \rightarrow M_{M}^{I I I}+1.5 V_{O}^{\bullet \bullet}+3 e^{\prime}
\end{gathered}
$$

$\mathrm{F} / \mathrm{S}$ interface

$$
\begin{aligned}
& M_{i}^{\bullet \bullet} \rightarrow M_{a q}^{X+}+(x-2) e^{\prime} \\
& 1.5 H_{2} O+1.5 V_{O}^{\bullet \bullet} \rightarrow 1.5 O_{O}+3 H^{+}
\end{aligned}
$$

where

$m \quad$ - The atom in the metal phase underneath the oxide film

$M_{i}^{\bullet} \quad$ - The divalent interstitial cation in the oxide film

$V_{m} \quad$ - The metal atom vacancy in the underlying metal phase

$M_{M}^{I I I} \quad$ - The trivalent cation in the oxide film

$V_{O}^{* \bullet} \quad$ - The oxygen vacancy in the oxide film

$O_{O} \quad$ - The oxygen anion in the oxide film.

Further, the ionic defects are assumed to act as electron donors; that is, strong coupling occurs between electronic properties and ionic conduction in the oxide $[43,46,48]$. In the case of a low-field strength in the oxide, which is completely analogous to the PDM [43, 47], the following relations have been obtained between the parameters of the transfer function [Eq. (3)(7)] and physical quantities pertinent to the generation, transport and consumption of point defects [43].

Young impedance, equation (3) [43]: 


$$
\begin{aligned}
& C=\frac{\varepsilon \varepsilon_{0}}{L} \text { insulating layer } \\
& C=\frac{\varepsilon \varepsilon_{0}}{W} \text { semiconductor layer } \\
& p=\frac{1}{2 K L} \\
& K=\frac{F}{R T} \mathrm{E} \\
& \tau=\frac{\varepsilon \varepsilon_{0}}{\sigma_{d}} \\
& \sigma_{d}=\frac{F^{2} D_{e}}{R T} \frac{k_{g}}{k_{c}}
\end{aligned}
$$

Warburg impedance, Eq. (4) [14, 43, 48]

$$
\begin{aligned}
R_{W, A} & =\frac{R T}{8 F^{2} K D_{i}(1-\alpha) c_{i(M / F)}} \\
\tau_{W, A} & =\frac{1}{2 K^{2} D_{i}}
\end{aligned}
$$

The concentration of point defects at the interface at which they are generated can be expressed as [44-47]:

$$
c_{i(M / F)}=\frac{k_{g}}{k_{c}} e^{-2 K L}+\frac{k_{g}}{2 K D_{i}}
$$

In these equations, $L$ is the thickness of the layer in which solid-state transport of point defects takes place under the influence of both potential and concentration gradients, $\mathrm{W}$ is the thickness of the depletion layer, $\boldsymbol{E}$ is the electric field strength, $D_{e}$ is the diffusion coefficient of electronic current carriers, $k_{g}$ and $k_{c}$ are the interfacial rate constants of generation and consumption of ionic current carriers, $D_{i}$ the corresponding diffusion coefficient of ionic point defects (vacancies or interstitials), $c_{i(M / F)}$ is the concentration of point defects at the $\mathrm{M} / \mathrm{F}$ interface and $\alpha$ is the polarizability of the $\mathrm{F} / \mathrm{S}$ interface [50, 52-54].

It has been previously demonstrated $[36,37,43]$ that the potential drop at the $\mathrm{M} / \mathrm{F}$ interface is independent of the external applied potential in the steady state. In other words, only 
the rate constant of consumption of the ionic current carrier at the F/S interface depends on the applied potential:

$$
\begin{aligned}
& k_{c}=k_{c 0} e^{b_{c} \alpha E} \\
& b_{c}=\alpha_{c} n_{c} \frac{F}{R T}
\end{aligned}
$$

where $\alpha_{c} \quad$ - transfer coefficient, and $n_{c}$ - number of transferred electrons.

\section{Kinetic Models for the Symmetrical Configuration}

The impedance response of a mixed conducting oxide sandwiched between the substrate metal and an inert metal probe can be interpreted on the basis of several models. Two of them deserve special attention - the homogeneous film model of Vorotyntsev and co-workers [23, 24, 52, 53] and the network model of Jamnik and Maier [54-57]. The oxide film is assumed to be a

mixed conductor with a single ionic and a single electronic current carrier. The following relations between the parameters of the Warburg impedance in the symmetrical configuration [Eq. (8)] and the physical quantities characterizing the defect transport are obtained in the limit of predominant electronic conductivity of the oxide:

$$
\begin{aligned}
& R_{W, S} \approx L \frac{R T}{z_{i}^{2} F^{2}} \frac{D_{i}}{D_{e}^{2} c^{0}} \\
& \tau_{W, S} \approx \frac{L^{2}}{4\left(z_{i}^{2}+1\right) D_{i}}
\end{aligned}
$$

Electronic conductivity is supposed to dominate in the hydrothermal oxide film because of its spinel-like structure $[58,59]$, and thus the electron exchange between cations in different valence states located in octahedral positions in the cation sublattice is relatively easy. Because the ionic carriers are assumed to be generated at the inner interface and consumed at the outer interface, a steady state profile of these defects exists in the film. It can therefore be assumed that the transport rate will be the slowest close to the film/solution interface where the concentration of defects is minimal. As a first approximation, in accordance to the PDM and the MCM [44-46], the steady state concentration of current carriers is:

$$
c^{0} \approx c_{i(F / S)}=\frac{k_{g}}{k_{c}}
$$

In summary, we intend to describe the processes in the oxide in terms of:

- Two rate constants, $k_{g}$ and $k_{c}$ 
- Two diffusion coefficients, $D_{e}$ and $D_{i}$

- Field strength in the oxide, $\boldsymbol{E}$

- Thickness of the layer in which point defect transport occurs, $L$

- Polarizability of the oxide/electrolyte interface, $\alpha$.

\section{FRACTURE SURFACE ANALYSIS}

The next step toward understanding the fundamentals of cracking phenomena is to correlate CDE results with crack growth data obtained under identical water chemistry conditions in fracture experiments. We use preloaded compact specimens of special design, which are exposed to the same conditions as CDE samples. Information on crack initiation and propagation mechanisms will be obtained by using SRI's FRASTA technique [60-66] to analyze conjugate fracture surfaces of test specimens.

The fracture surfaces of broken specimens contain a record of the details and sequence of the microfracture process that caused the crack to propagate, and can yield more information provided that proper examination and analytical techniques are used. Fracture surfaces of test specimens are routinely examined using an optical microscope and scanning electron microscope to obtain qualitative information, including crack initiation sites, the direction of crack propagation, and associated failure mechanisms. While it is also possible to obtain limited quantitative information (such as fatigue crack growth rates based on striation spacing) by using conventional techniques, generally there is not sufficient information for use in quantitative fracture analysis.

SRI has developed a new fractographic analysis technique, FRASTA, or fracture surface topography analysis, that overcomes the limitations of conventional techniques by quantifying and analyzing the topographies of conjugate fracture surfaces simultaneously to reconstruct a fracture event in microscopic detail. The method involves a FRASTAscope, which combines a confocal-optics-based scanning laser microscope and computer software to match threedimensional features of conjugate fracture surfaces and reconstruct the detailed fracture process that occurred inside the material [60]. FRASTA provides critical information on crack nucleation and growth in the absence of test instrumentation (i.e., without the need for fracture toughness tests) [61]. This capability is especially valuable in light of the extreme conditions of tests in supercritical water.

The concept of analyzing conjugate fracture surfaces is based on accurately characterizing the microfailure process that results in a final rupture (Figure 9). For example, a structure deforms elastically after it is first loaded, and upon further loading, localized inelastic 
deformation occurs at defects or at sites of geometrical stress concentration. Upon further loading, a microcrack develops within or near the inelastic zone. Stresses on the newly formed microfracture surfaces (free surfaces) fall to zero. Thus, the material immediately beneath the surfaces "freezes in" the deformation that existed at the moment of cracking and undergoes no further inelastic deformation. The applied load is redistributed to sound material in front of the crack tip, which continues to deform inelastically until it too fractures.

FRASTA has been used to determine crack initiation times and crack growth rates from post-test examination of AISI Type 304 stainless steel constant extension rate test (CERT) specimen fracture surfaces [60]. Furthermore, FRASTA made it possible to clearly identify the difference in microfracture mechanism between two specimens tested in different environments (Figure 10), an unexpected difference that could not be determined simply by microscopic examination of fracture surface features.

The sensitized Type 304 CERT specimens were tested in different simulated, modified BWR service environments: one in clean water (conductivity $<0.1 \mu \mathrm{S} / \mathrm{cm}$ ), and the other in water deliberately contaminated with $1 \mathrm{ppm}$ sulfuric acid. The water temperature in both tests was $290^{\circ} \mathrm{C}\left(550^{\circ} \mathrm{F}\right)$. The CERT strain rate was $7 \cdot 10^{-8} \mathrm{sec}^{-1}$ over a test period of 1 week.

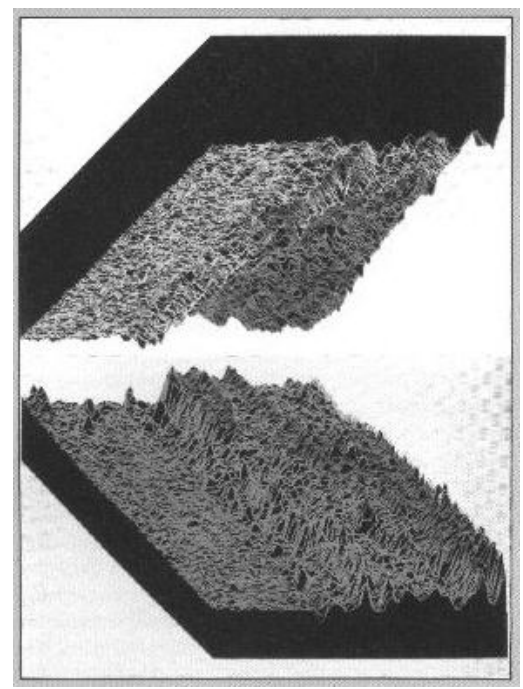

Topography surfaces

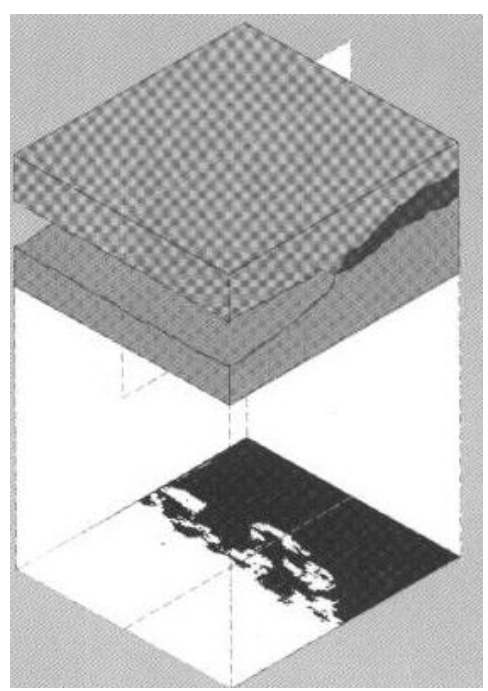

Fractured area projection plot

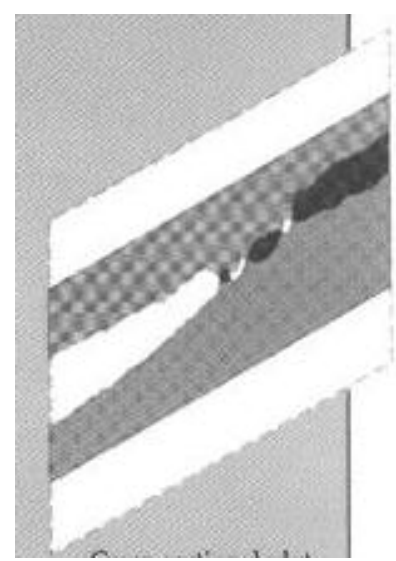

Cross-sectional plot

Figure 9. Use of a confocal-optics-based laser microscope (SLM) in the FRASTA technique makes it possible to characterize surface topography quickly and accurately (left top and bottom). FRASTA results are presented as fractured area projection plots, which provide information on microcrack initiation sites and projected areas of micro/macrocracks; and as cross-sectional plots, which show the microcracks in profile, the macrocrack tip opening angle and displacement, and the amount of inelastic deformation necessary before fracture [60]. 
The sequential process produces different amounts of local inelastic deformation in the material as a function of distance from the microfracture nucleation site. The difference in the local inelastic deformation results in a surface irregularity (i.e., an elevation profile on the fracture surface), thus providing a record of the history of microfracture events.

However, the crack itself interacts with the local microstructure, and the resulting weaving crack path also produces surface irregularities. It is necessary to separate the two surface irregularities, which can be accomplished by comparing conjugate fracture surface profiles using FRASTA. If the irregularity is caused only by interaction of the crack tip with the microstructure and without inelastic deformation, the conjugate surface profiles should match precisely. Any mismatch appearing as overlap between the conjugate surface profiles indicates inelastic deformation. The amount of local inelastic deformation that occurred prior to fracture is determined with the aid of a computer. The difference in the local inelastic deformation is used to reconstruct the microfracture sequence from beginning to end.

Figure 10 shows a series of fracture area projection plots (FAPPs) of the notched section in a direction parallel to the specimen axis at various relative displacements of the topographic maps. Areas where topographs overlap (shown in black) are considered intact material; those that do not overlap indicate cracks (shown in white). The plots show that cracking initiated at the surface (notch root) at two locations and grew inward for specimens tested in pure water (Figure 10, left column). For specimens exposed to low $\mathrm{pH}$ water (Figure 10, right column) crack initiation and growth started in the interior of the specimen. Internal cracks merged with the surface only after considerable growth, followed by a rapid decrease in unfractured area. This cracking mode is markedly different from that observed in the specimen tested in clean water. FRASTA was able not only to distinguish between two different cracking mechanisms but also to estimate crack initiation times and crack growth rates, as described in detail in [60].

This example shows FRASTA's unique ability to reveal the entire sequence of evolving fracture events. This capability makes FRASTA not only attractive but irreplaceable for investigation of cracking modes under the new conditions, which will exist in the heat transport circuit of Generation-IV SC LWRs. In combination with information on the role of oxide films on the surface of the structural components, FRASTA results will provide an in-depth understanding of the degradation mechanism and its driving forces. 
Pure BWR water

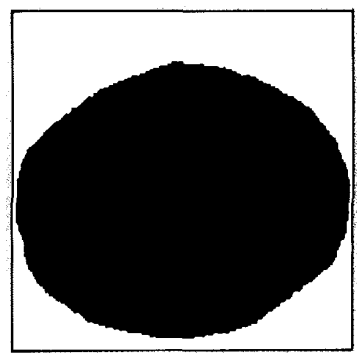

(a) 0 hours

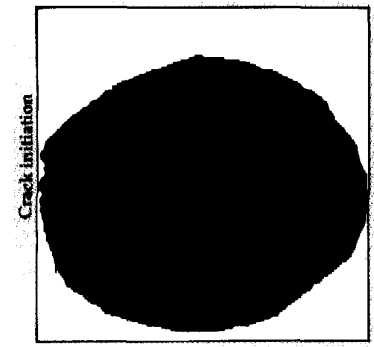

(b) 90 hours

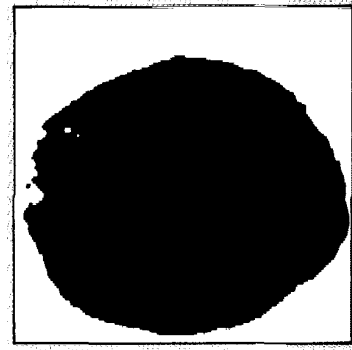

(c) 110 hours

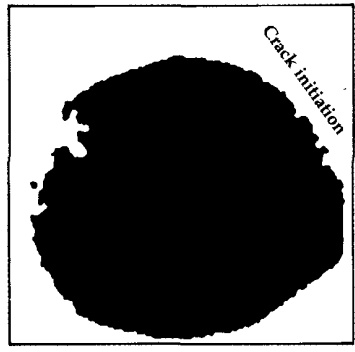

(d) 130 hours

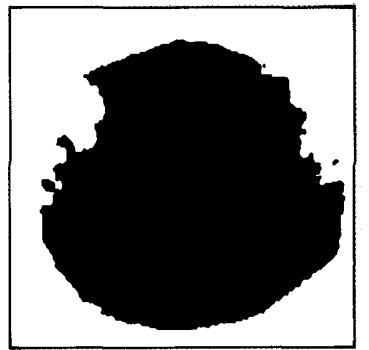

(e) 150 hours
BWR water with $1 \mathrm{ppm}$

sulfuric acid
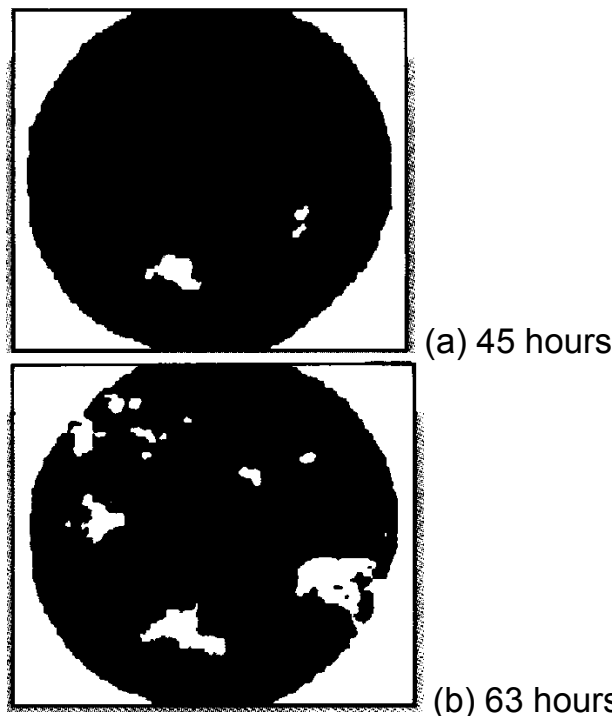

(b) 63 hours
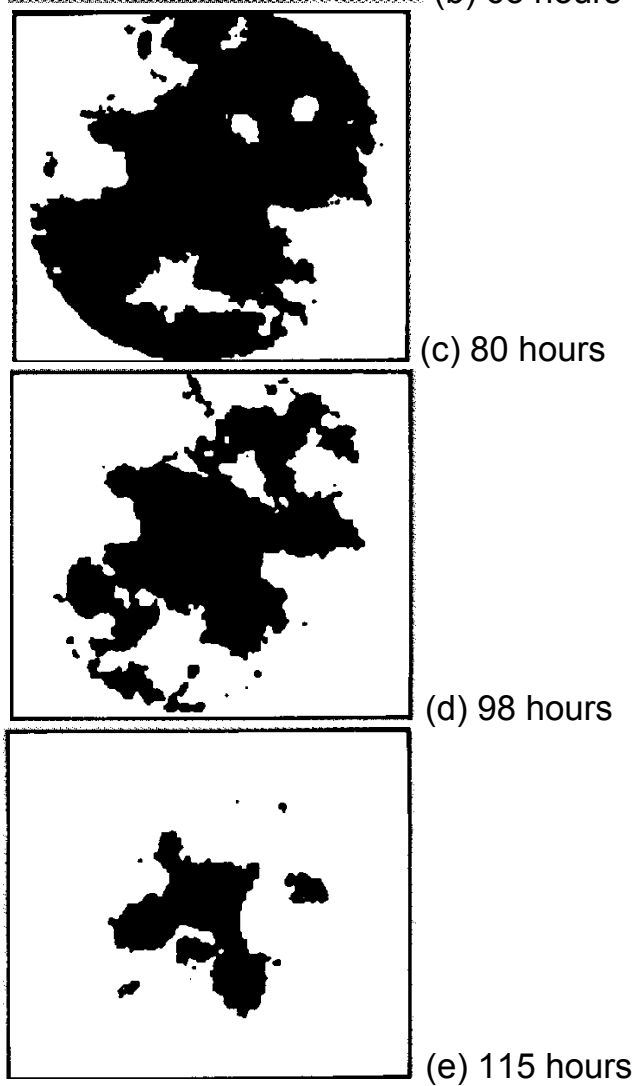

Figure 10. Series of fractured area projection plots for Type 304 CERT specimens tested in simulated pure BWR water (left column) and in BWR with $1 \mathrm{ppm}$ sulfuric acid (right column). Black area indicates intact material; white area, cracks [60]. 
Fracture surface analysis included:

- Pretreating the surface to remove corrosion products

- Obtaining a 3-D topography map of the conjugate fracture surfaces using a confocal laser microscope.

- Processing the fracture surface topography to indicate information on crack propagation.

- Estimating the crack growth rates.

\section{Pretreatment of the Fracture Surfaces}

The corrosion products on the fracture surfaces were removed by an electrochemical oxide removal procedure developed by Yuzawich and Hughes [67], using Enprep 114 E made by Enthone-OMI Inc. A solution was prepared by dissolving $120 \mathrm{~g}$ of Enprep 114E and $90 \mathrm{~g}$ of sodium cyanide in $1 \mathrm{~L}$ of water. A small amount of Photoflo was also added to the solution.

A glass beaker containing $500 \mathrm{ml}$ of the solution was placed in an ultrasonic cleaner. A Pt-clad Ti-Nb mesh was placed along the inside wall of the beaker and connected to the anode terminal. The specimen was connected to the cathode terminal and immersed in the solution at the center of the beaker. A current density of about $180 \mathrm{~mA} / \mathrm{cm}^{2}$ was applied for $2 \mathrm{~min}$. The Yuzawich and Hughes procedure calls for the application of $250 \mathrm{~mA} / \mathrm{cm}^{2}$, but we could not apply higher current because of the limitations of the equipment. The specimen was then removed from the solution, washed in clean water, dipped in methanol, and dried with compressed air. The fracture surface was examined visually to ascertain whether or not the corrosion products had been satisfactorily removed. If the oxide had not been removed, the procedure was repeated.

Figure 11 shows the SEM photographs of the same fracture surface of 304 No. 3 specimen before and after electrochemical cleaning. In the fracture area on the left half of the photograph, the crack extension occurred in the supercritical water environment and after long exposure to the environment. The right half of the photograph is the area where forced crack opening by fatigue cycling occurred; that area is the freshly fractured area.

The fractured area where long exposure to the environment occurred was covered by the oxides. White areas in Figure 11a were covered by needle-like oxides, and dark areas were covered by globular-shaped or smoother-surfaced oxides. Figure 12 shows the detailed features of oxides at a higher magnification. Two types oxides were observed: globular shaped in the dark areas and needle-like shaped in the white areas. Figure $11 \mathrm{~b}$ shows the fracture surface after electrochemical cleaning. The total cleaning time was $15 \mathrm{~min}$. The electrochemical cleaning process removed oxides well without damaging the base metal. Detailed examination and 
comparison of the forced-open fractured area before and after cleaning demonstrated that the electrochemical process did not attack or alter the fresh fracture surface area in any way. Thus, given that the Enprep solution is alkaline and does not attack the base metal, we can assume that the cleaning process removed only oxides.

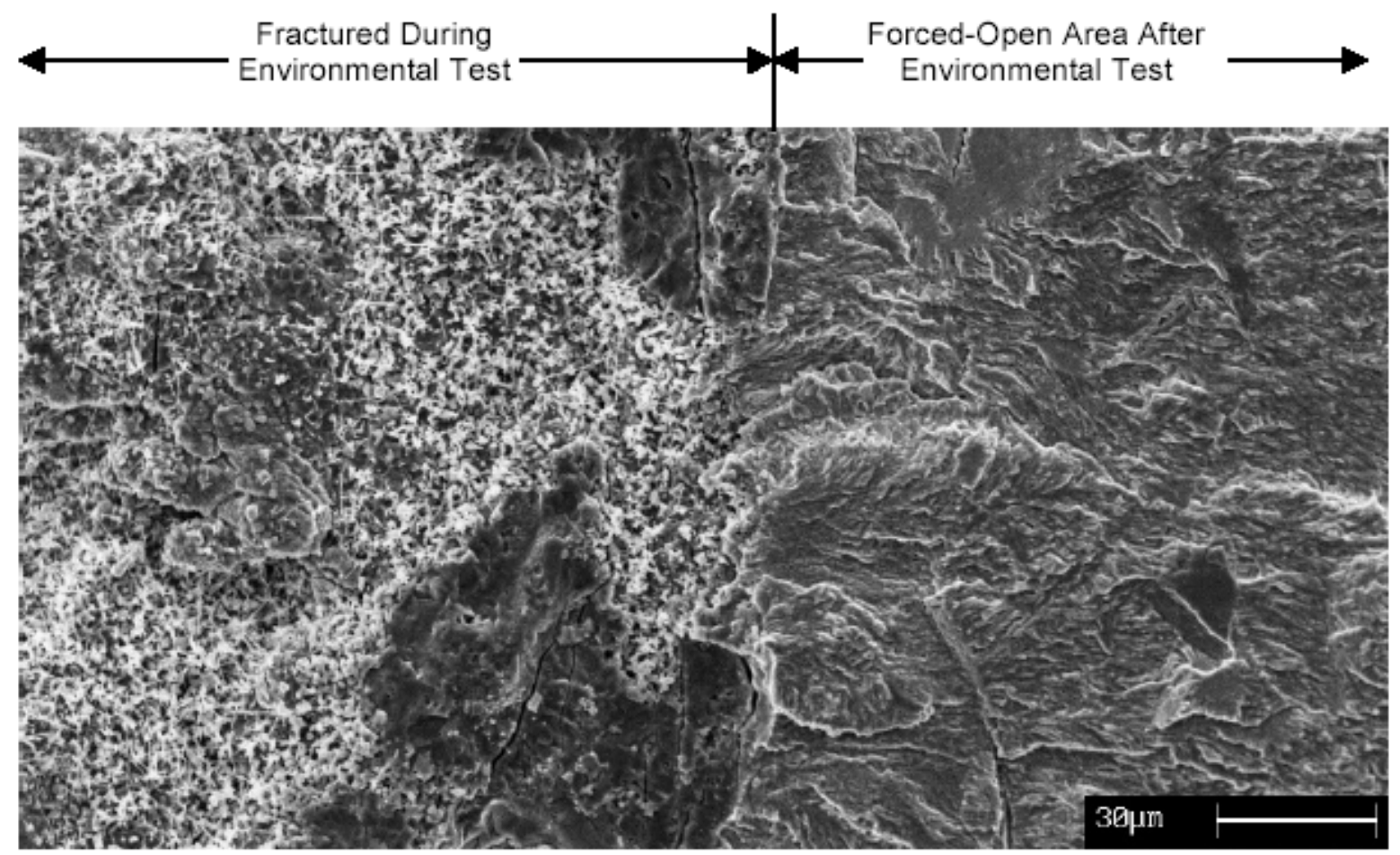

(a) Before Electrochemical Cleaning

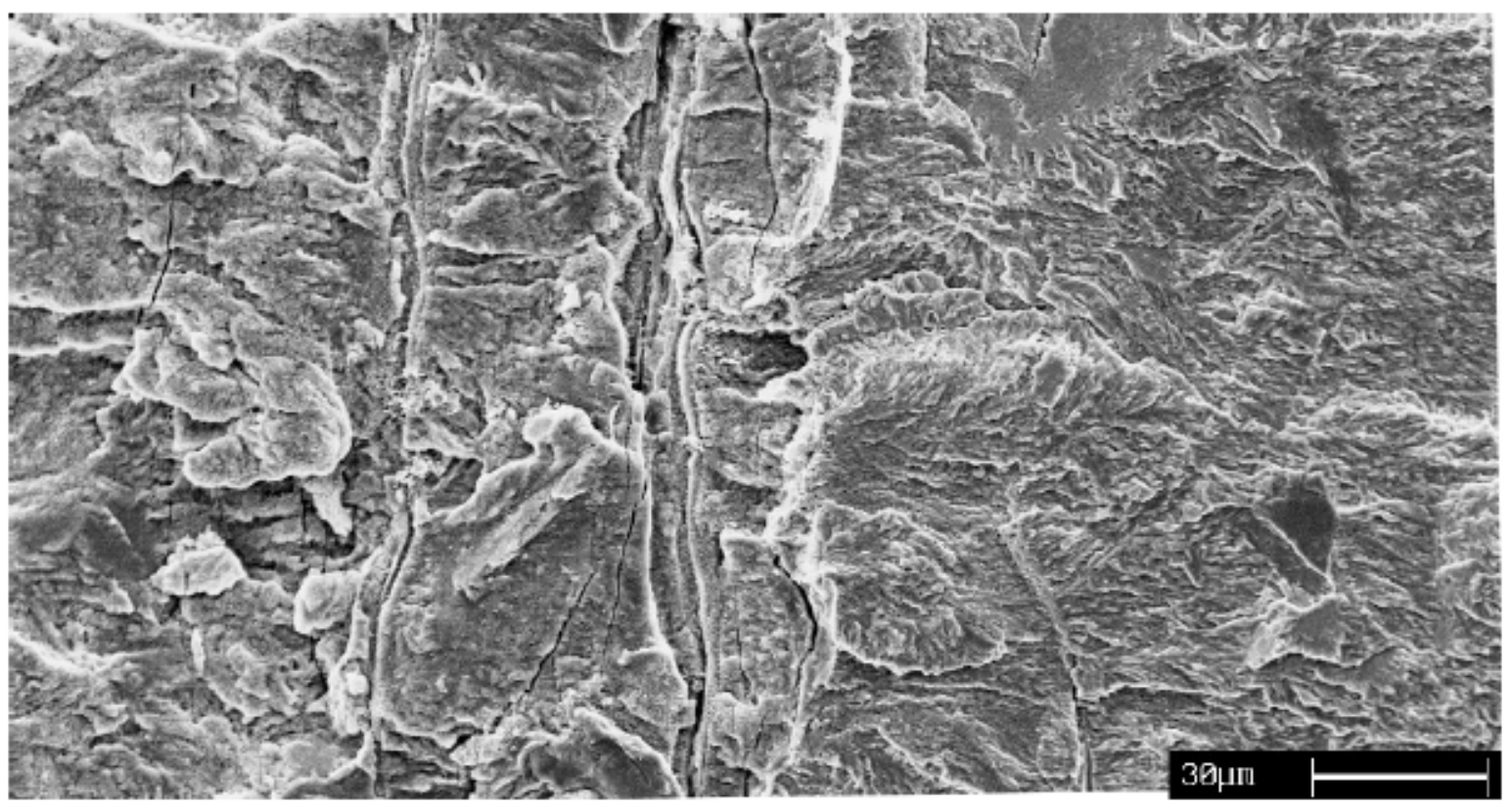

(b) After Electrochemical Cleaning 
Figure 11. SEM photographs of the same fracture surface area before and after electrochemical cleaning.

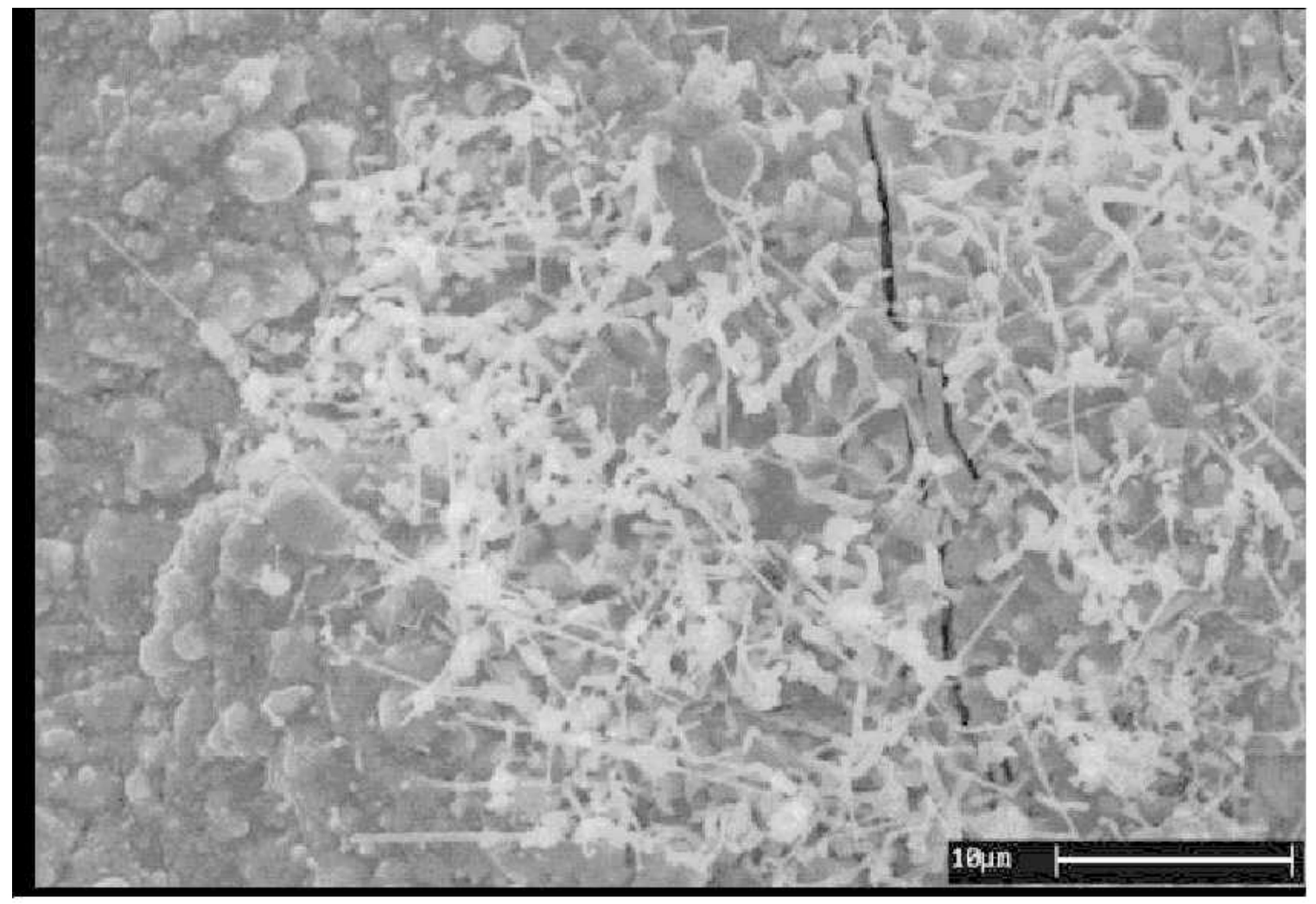

Figure 12. Higher magnification SEM photograph of the oxide-covered fracture surface.

\section{Obtaining a 3-D Topography Map of the Conjugate Fracture Surfaces}

The topography of fracture surfaces was characterized by SRI's FRASTAscope shown in Figure 13. The FRASTAscope consists of a confocal-optics scanning laser microscope (COSLM), with computer-controlled precision $\mathrm{x}-\mathrm{y}-\theta$ stages, a computer-controlled precision $\mathrm{z}-$ stage, and computer software that controls the system and creates the digital files.

The observation area size of the COSLM is small $(137.5 \mu \mathrm{m}$ by $93.6 \mu \mathrm{m}$ with an $80 \mathrm{X}$ objective lens and $223.3 \mu \mathrm{m}$ by $150.5 \mu \mathrm{m}$ with a $50 \mathrm{X}$ objective lens). For most of the fracture surface studies, one observation area of the COSLM is not enough. The FRASTAscope can patch many observation areas to cover a larger fracture area and create a single data file automatically. The ultimate elevation resolution of the FRASTAscope is $\pm 60 \mathrm{~nm}$, and the maximum elevation characterization range is $2 \mathrm{~mm}$. The COSLM, however, is an 8-bit system, and the normal mode of operation is to divide the specified elevation range into 256 divisions. The accuracy of the measurement of each elevation step is $\pm 0.2 \%$ of the full characterization range. 
In the FRASTA examination, precise matching of conjugate fracture surfaces is important. We were able to match the conjugate surfaces precisely by comparing the conjugate surface images on the computer monitor and adjusting the matching surface position with a resolution of $\pm 0.1 \mu \mathrm{m}$. Figure 14 shows an example of the fracture surface topography produced by FRASTA.

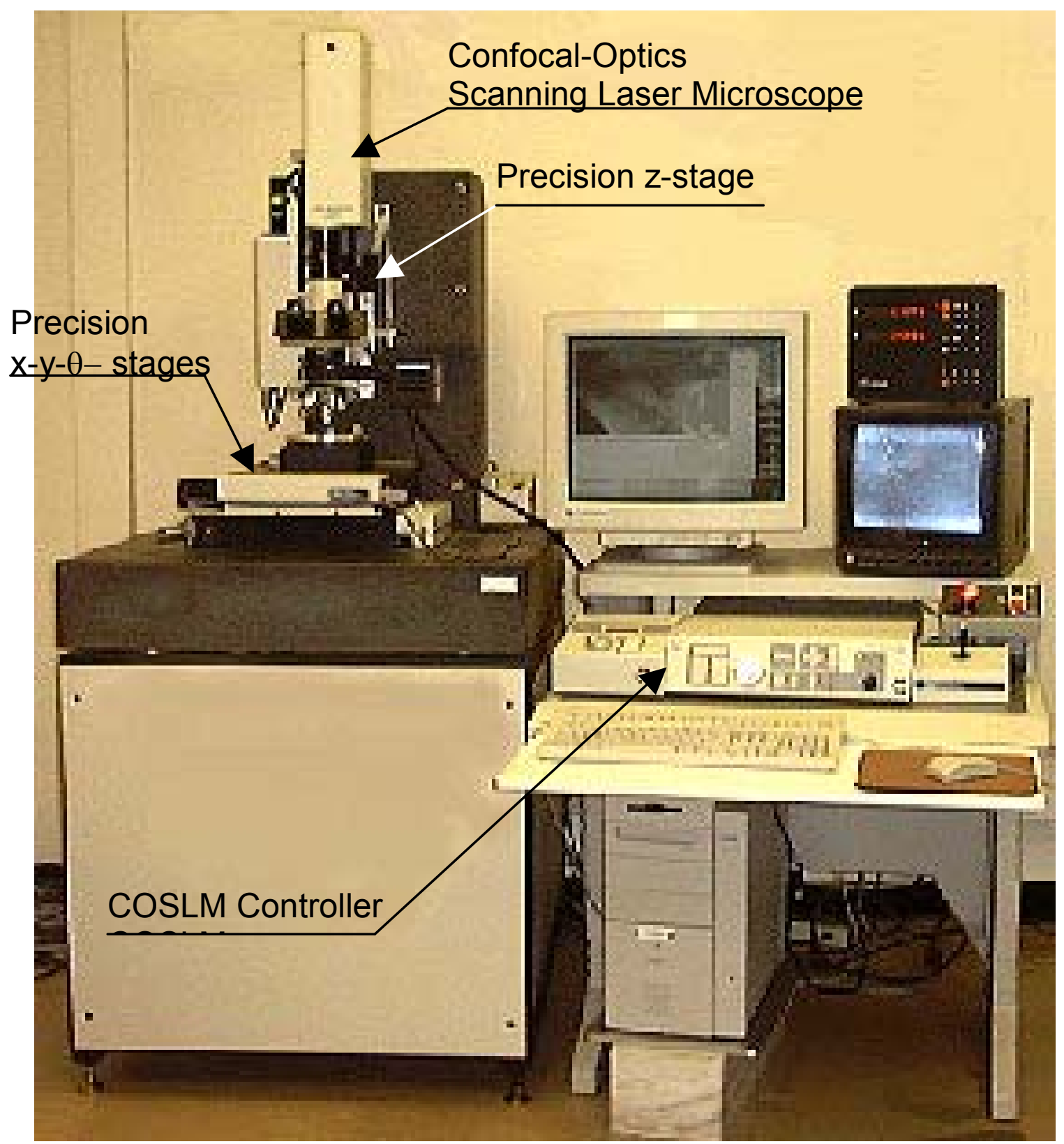

Figure 13. FRASTA instrumentation. 


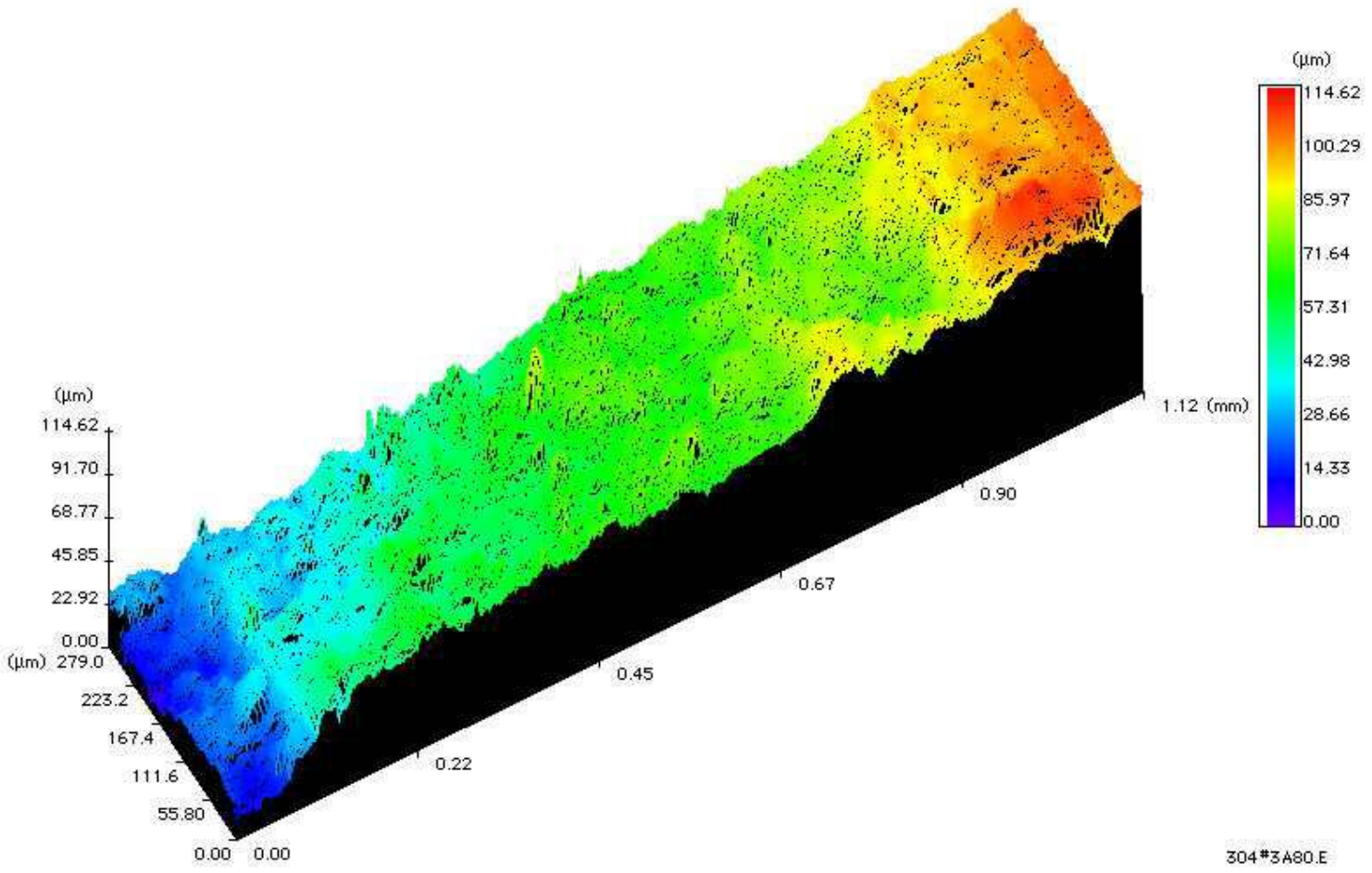

Figure 14. An example of the 3-D topography map of the fracture surface.

\section{Unfolding Crack Propagation Information}

The conjugate topographic data files (Surface A and Surface B) were input to the reconstruction program. The data file Surface B was then flipped and juxtaposed over the Surface A so that the one-to-one match of surface positions area was established.

The position of the Surface B with respect to the Surface A was then changed so that the initial overlap of conjugate surfaces occurred over the entire area. From this state, Surface B was lifted incrementally against Surface A. After each incremental separation, the entire area was scanned to determine if a gap between two surfaces had occurred. If a gap(s) between the conjugate surfaces was detected, their positions were plotted in the observation area window. This plot is called Fracture Area Projection Plot (FAPP). The FAPPs can be considered similar to the $\mathrm{x}$-radiograph of internal defects. Figure $\mathbf{1 5}$ provides an example of the FAPP superimposed on an SEM image of the fracture surface. 


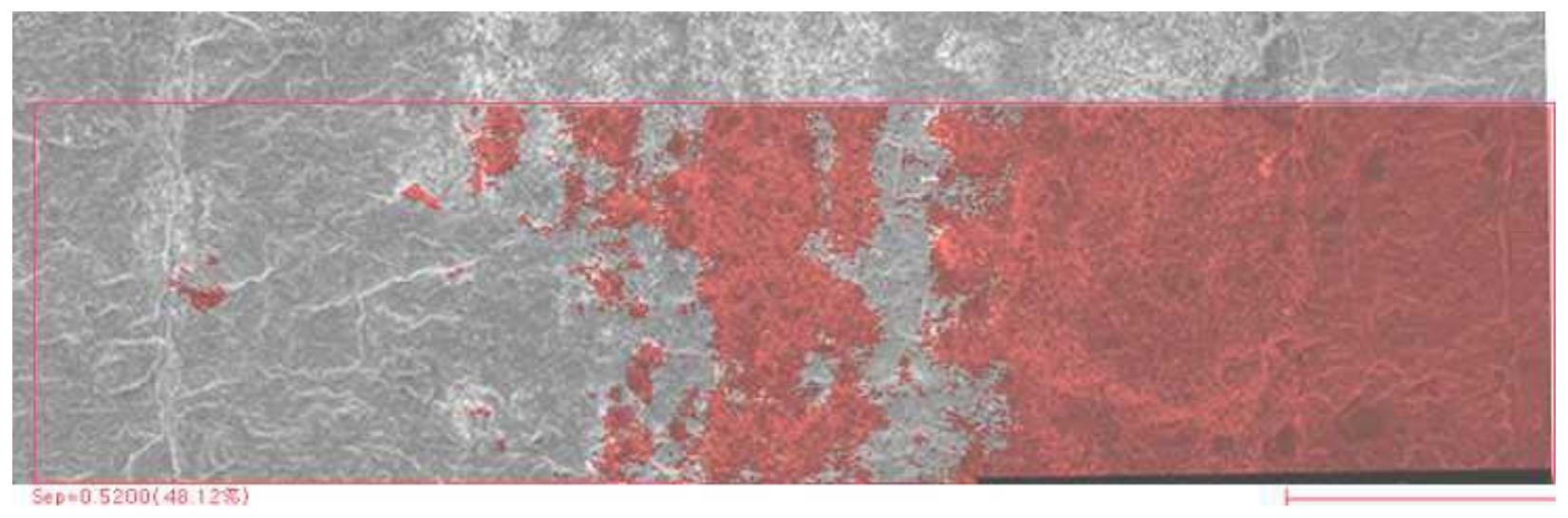

Figure 15. Fractured Area Projection Plot superimposed on an SEM image of the fracture surface.

In constructing FAPPs, it is important to consider that, with crack growth under loaded condition, the material in the vicinity of a crack tip is in a deformed condition and the crack is in an opened state with a certain angle. However, when the fracture surface topography was characterized after the test, the specimen halves were unloaded, and elastic recovery occurred. Thus, it was necessary to simulate the crack opening when we matched the conjugate surfaces. To do so, we tilted both surfaces so that the surface behind the crack tip formed an angle similar to the crack-tip opening angle during the crack growth. The FRASTA reconstruction program can implement tilting of the conjugate topography data and produce FAPPs.

FRASTA's advantages include generating FAPPs at desired incremental spacing and observing not only the movement of a crack front, but also the details of fracture process in a vicinity of a crack front (e.g., formation of advanced microcracks).

FAPP can be superimposed on the fracture surface photographs (e.g., SEM, see Figure 15 as an example) and used to transfer the crack front locations or microfracturing locations in the FAPP onto the fracture surface. Such correlation can help establish microstructural roles in fractures.

\section{Estimation of Crack Growth Rates}

A series of FAPPs can show the movement of the crack front through the specimen; however, any changes in the crack growth rate is difficult to detect. We obtained information about changes in the growth rate by computing the gapped (white) areas in the FAPPs and plotting them as a function of conjugate surface spacing. This plot is called a fracture progression curve (FPC). FPC is sensitive to detecting changes in the crack growth rate due to factors such as changes in load, environment, or mechanism. We used this curve to determine the crack front position before the environmental test. Crack growth rates were estimated using 
information from FPC and the "time marks" procedure we developed during the first year' of the project [3].

\section{CORRELATION OF THE OXIDE FILM STABILITY AND CRACK GROWTH}

\section{Multi-Disciplinary View of Stress Corrosion Cracking}

Stress corrosion cracking (SCC) is a phenomenon where the combined effect of mechanical loading, material composition and environment conditions leads to initiation and propagation of cracks until potential failure of a component or structure. It is hardly a central phenomenon in a particular discipline, but is still a challenging subject to a number of various disciplines - electrochemistry, mechanics, metallurgy and physics.

The challenge comes from the ease with which SCC deteriorates nominally tough and corrosion resistant materials. The study of SCC involves such a wide spectrum of knowledge that no scientist at present could cover all the aspects with equal expertise. Engineering applications require a research output either in terms of measures to avoid SCC, or in terms of components durability. In both cases there is a need for some predictive models relating local parameters, which drive the crack evolution, to the global parameters, like bulk environment, applied loading and material properties. Development of predictive models for SCC relies on two separate stages. Firstly, a mechanism for the process of crack growth should be established and the various factors that influence this process should be identified. The first stage would give a qualitative description of the phenomenon. Secondly, the qualitative description should be formalized in a quantitative mathematical framework.

There are numerous mechanisms proposed to describe SCC, based broadly on the effect of anodic dissolution and of absorbed hydrogen. The study of the latter has evolved into a separate research area, named hydrogen embrittlement (HE). Distinction between these two groups of mechanisms is not always easy. While anodic dissolution is a possibility in most metals and alloys, HE is not a practical problem with all of them. The ones most vulnerable to HE are titanium alloys, aluminum alloys and high-strength steels. When both mechanisms are probable, the environmental conditions and the metallurgical composition are responsible for choice of a dominant mechanism. A discussion on when and where the mechanisms based on anodic dissolution play a dominant role is outside the scope of this work, but may be found in [69-74] among others.

The most accepted mechanism for SCC based on anodic dissolution, is the film rupture mechanism. It is experimentally shown to be the dominant mechanism in intergranular stress corrosion cracking (IGSCC). The basic processes defining this mechanism will be described in 
brief. If a bare surface of a metal or an alloy, susceptible to corrosion, is put in contact with an aqueous solution a process of dissolution of metal ions starts. Some of the dissolved metal ions form oxides and hydroxides that precipitate on the surface, forming a protective film. This film is also called passive since it is almost inert and acts as a barrier for a continuing dissolution. The process of film formation is called passivation of the surface. After sufficiently long time the contact surface may be compactly covered with an oxide film and the dissolution would practically stop. If the surface is deformed, however, the protective film may break and new bare metal may be exposed to the solution, paving the way for the process of dissolution. At the same time a repassivation of the surface begins. The film rupture mechanism considers crack growth as a series of discrete steps, where each step contains the rupture-dissolution-repassivation sequence. In order to maintain the growth, there must be an adequate cause for rupture in the beginning of each step.

One proposed cause for rupture is the strain rate at the crack tip, which has lead to the quantitative model by Ford [75-77]. The notion of crack tip strain rate is a consequence of the considered ideal crack geometry, where the crack tip is represented essentially as a point. In such a case the mechanics may contribute to fracture via a single parameter - e.g. the stress intensity factor, or the crack tip opening displacement, or the J-integral - and the strain rate at the tip is expressed as a function of the chosen one. Ford's model is perhaps the most widely used predictive tool at present, but still there is a continuing discussion about the model electrochemical and mechanical parameters identification.

In the majority of studies on SCC by anodic dissolution, much thought has been given to the precise role of the corrosion process itself. Some examples are the works by Danielson [78], Macdonald and Urquidi-Macdonald [79], Simonen [80], Turnbull [81], and Engelhardt et al. $[82,83]$. All these works rely on the Ford model to account for the mechanical contribution to SCC phenomenon. The crack is assumed existing with a certain length. The emphasis is put on the determination of the localized chemistry of the solution near the crack tip. Thus, the influence of various parameters, such as $\mathrm{pH}$ of the solution, the concentration of aggressive anions and oxygen, and the temperature, has been investigated. Transport processes, like diffusion, convection and electro-migration have been considered to improve the prediction for the near-tip solution composition and electric potential difference.

An essential question, not answered in the electrochemical studies, is why in reality the dissolution occurs over such a relatively small region so that the crack shape is maintained. This question cannot be answered keeping the assumption of ideal crack geometry. This is because important information about the dissolution process is hidden and the crack shape maintenance is already enforced. If the dissolution front were broad, one would expect development of a pit, 
rather than a crack, according to the electrochemical models. Obviously, crack growth by anodic dissolution requires some critical balance between active and passive behavior, such that the dissolution at the tip proceeds at a considerably faster rate than at any other place where it may occur. Probably to a large extent, that critical balance is determined by the different deformation states of the points along the corroding surface. This question is even more pronounced when it comes to crack formation from surface pits, as observed in experiments and reported by Chen [84], Rokhlin [85], Pao [86], Murtaza and Akid [87] and Sankaran [88], among others. All these works show that mechanics is involved in the pit evolution to crack formation from the very first stages of the process.

\section{Role of Repassivation in Crack Evolution}

It must be realized that the role of the protective surface films and repassivation phenomena are of overall critical importance in this respect. This is because they control the accessibility of the solid to the solution. Then comes the description of the dissolution kinetics, with the main controlling factors being investigated in the electrochemical works. The dissolution kinetics would provide the rate of corrosion and thus the crack evolution, in case there is accessible bare metal to be dissolved. The repassivation kinetics would introduce a time scale by limiting the period of active dissolution. What would be an important mechanical contribution, therefore, is to investigate the interaction between the applied load and the protective film.

Another important question arises from the available experimental data on SCC, which does not always confirm the predictions of electrochemical models. A compilation of such data may be found in [89]. The compilation reveals a tremendous scatter, up to five orders of magnitude, in the measured crack growth rates for the same applied loads. Suggested explanations for that scatter are different test durations and insufficiently controlled environmental conditions and load applications. The question here is, if there are factors of mechanical or geometrical nature that may lead to a scatter in the observed growth rates. Examples are length and shape parameters introduced by the metallurgical composition and the loading history. In order to investigate such a possibility, it is again necessary to abandon the ideal crack geometry, and assume a cavity-like configuration, which will be called a realistic crack. At large enough distances from the tip the configuration can still be considered as a classical crack, but at small distances it is rather a notch. The separation line between these two regions may be defined through the $\mathrm{K}_{I}$-controlled stress field in the outer region.

The rate of penetration in the material, due to dissolution at the anode, may be calculated using Faraday's law as 


$$
v_{0}=\frac{M}{\rho} \frac{i_{a}}{n F}
$$

where $\mathrm{M}, \rho$, and $i_{a}$ are the atomic weight, density of the dissolving metal, and anodic current density respectively.

In most cases, however, the penetration rate given by (Eq. 27) is not the rate that would be observed in a corrosion experiment. The actual penetration rate, $v$, would be less then $v_{0}$ if the availability of reactants at one or at both anodic and cathodic sites is limited. Hence, $v_{0}$ represents the maximum possible rate at the given potential. Generally, there are two phenomena that control the reactants availability - passivation and mass transport. Passivation is principally important at the anode, while mass transport limitation dominates at the cathode.

Some metals produce insoluble metal compounds, for example oxides, able to become attached to the metal surface forming a protective coat against further corrosion. The metal then becomes immune, even if corrosion is thermodynamically very favorable, because the supply of the anodic reagent is blocked. This process is called passivation and the protective coat is often called passive, protective or oxide film. The passive film is usually a brittle ceramic-like formation. For metals and alloys, that form protective films it has been observed experimentally that the actual penetration rate, $v$, has initially high value, $v_{0}$, for a short time, $t_{0}$, and thereafter the passivation leads to a decay that follows a power low dependence on time (see e.g. Ford [77]). This observation is summarized in

$$
v=v_{0}\left(\frac{t_{0}}{t}\right)^{p}, t \geq t_{0}
$$

where $t_{0}$ is a "repassivation time" ( $p$ is approximately 0.5$)$.

One of the contributions of this NERI project to fundamental understanding of SCC is ability to measure repassivation time directly using in-situ CDE technique. Another contribution is a suggestion that $p$ is a function of the oxide film stability and may be derived form CDE experimental data on transport properties of the oxide films. Impact of oxide film stability on crack growth for a give repassivation time is illustrated by Figure 16.

The second factor that may influence the corrosion rate is the supply of cathodic reagent. If it cannot be maintained at the levels, required to keep the electrochemical reaction going at certain rate, the mass transport of that reagent becomes a rate-controlling factor. The cathodic current then decreases, which correspondingly decreases the dissolution at the anode, because the consumption of electrons falls. The process may be viewed as a reduction of the applied overpotential. 


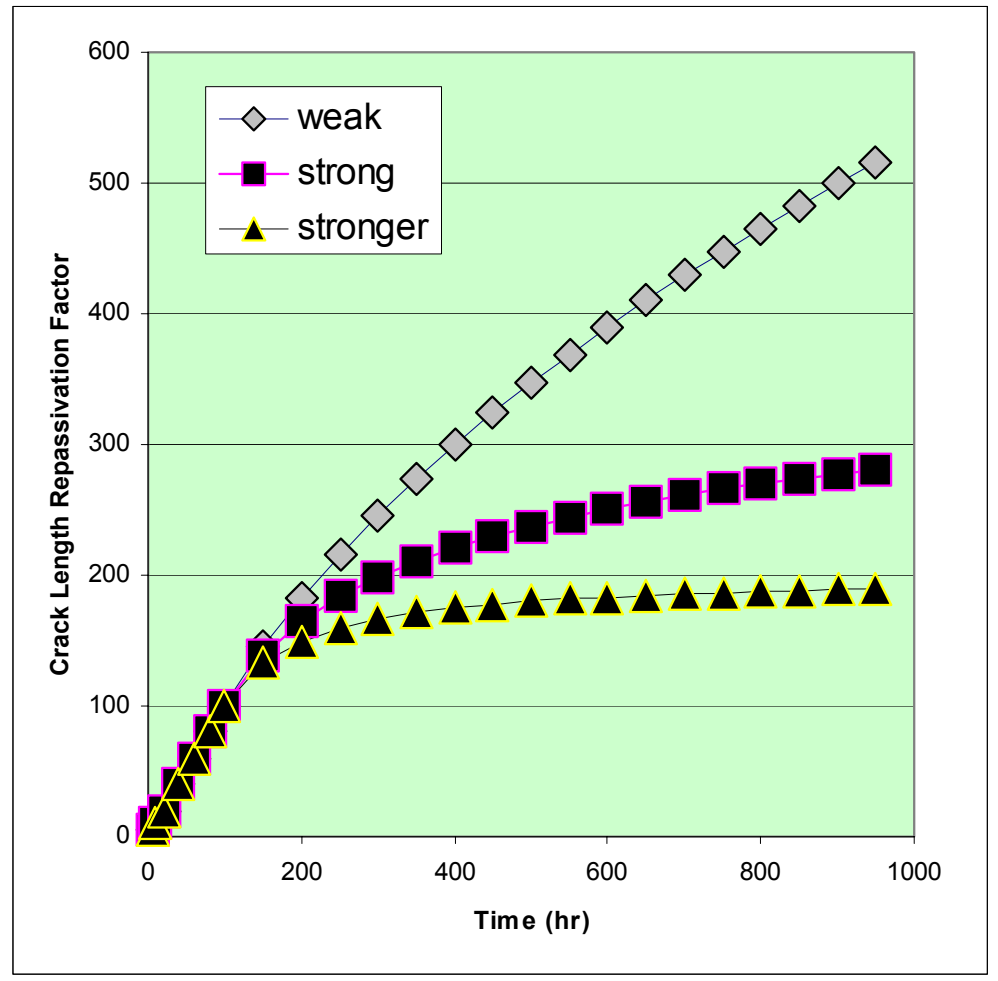

Figure 16. Impact of the oxide film stability on the SCC crack propagation. "Stronger" denotes more stable film (higher resistivity to charge and mass transfer)

\section{A Step-Wise Crack Advance Mechanism}

Consider (Eq. 28) as giving the time variation of the dissolution rate, when both passive film formation and transport control are taken into account. As discussed in the previous section, the maximum dissolution rate, $v_{0}$, has a precise physical interpretation, while the characteristic time, $t_{0}$, and the exponent, $p$, incorporate the influence of the passivation and the charge and mass transport limitations. According to this equation, if a bare metal surface is brought in contact with a corrosive environment, a short-term dissolution process with a maximum rate would initially be observed. A long-term process of transport and passivation limited dissolution would follow with a rate decaying down to the process eventual stop. Such a development may be altered through a mechanical loading. If the deformation of the surface is sufficient to rupture the protective film created by passivation, new bare metal would be exposed to the environment and the dissolution would be maintained. The growth of corrosion cracks is presented as a sequence of rupture-dissolution-passivation steps. Such step is illustrated in Figure 17. In the figure, $\delta$ is the linear extent of the active region for dissolution. It is assumed equal either to the inter-phase thickness as in Figure 18, or to the crack tip opening displacement 
if small scale yielding is considered. Further, the surface strain at rupture of the passive film is introduced as a material parameter and denoted by $\varepsilon_{f}$.

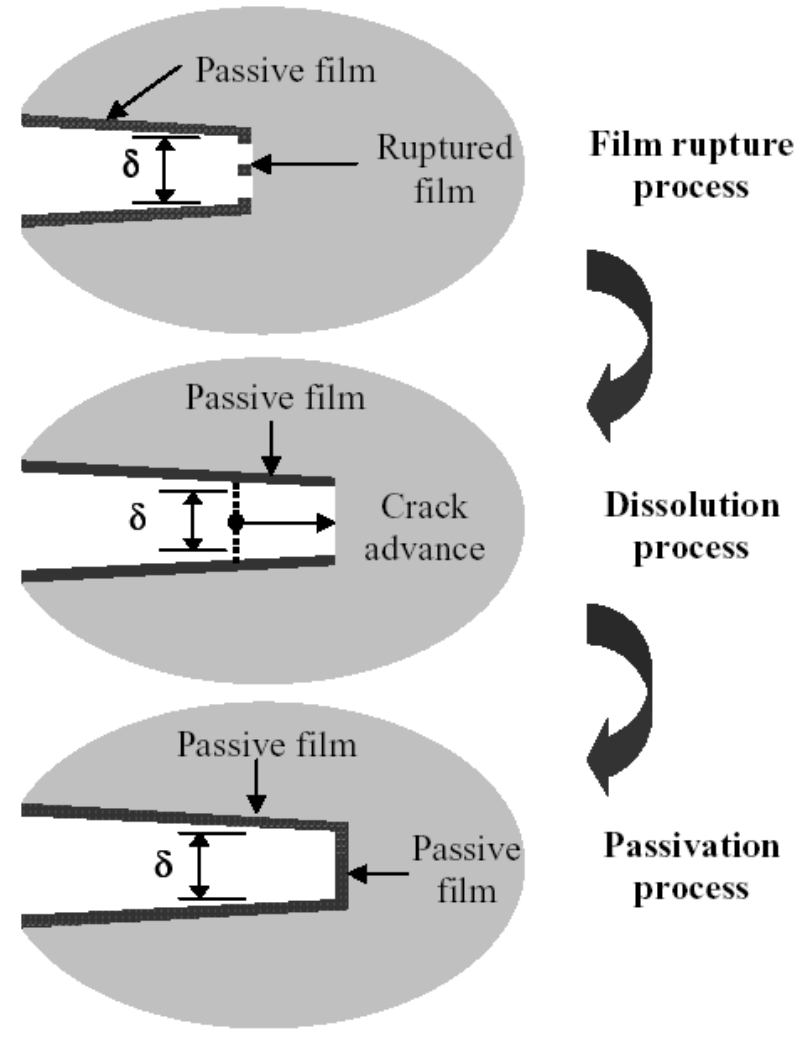

Figure 17. A step in the film rupture mechanism

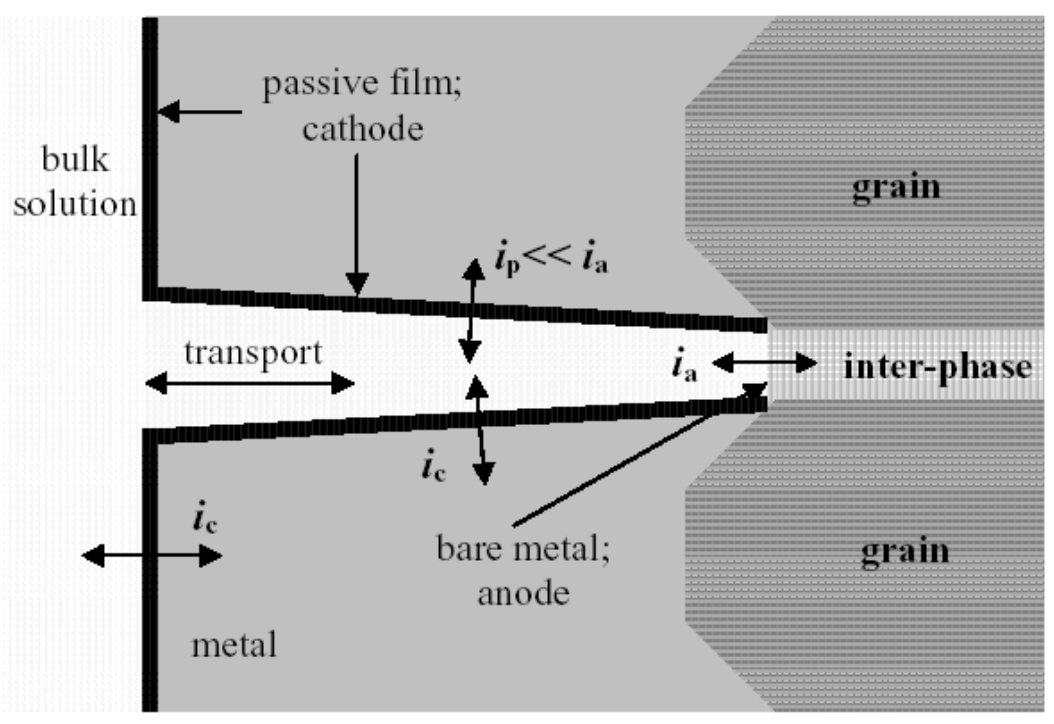

Figure 18. crack geometry with processes 
The rupture strain may be estimated experimentally [83]. The relation between $\varepsilon_{f}$, the actual state of deformation and the rate of dissolution is made by expressing the time variable in (2.3) as follows

$$
t=\frac{\varepsilon_{f}}{\dot{\varepsilon}}
$$

where $\dot{\varepsilon}$ is the strain rate at the crack tip, given in the sense of material derivative. Thus the crack growth rate $\dot{a}$, is derived from (Eq.29) as

$$
\dot{a}=v_{0}\left(\frac{\dot{\varepsilon}}{\dot{\varepsilon}_{f}}\right)^{p}
$$

where the term

$$
\dot{\varepsilon}_{f}=\frac{\varepsilon_{f}}{t_{0}}
$$

is introduced for convenience and sometimes called rupture strain rate.

Eq. 30 constitutes the mechanistic model by Ford. As a material derivative, the crack tip strain rate consists of two terms - a local strain rate due to external factors and a convective strain rate due to crack advance. Ford defines the local strain rate through the applied load rate in case of active loading, and through the rate of creep in case of constant load [75]. By active loading it is meant a time-varying load application, either cyclic or non-cyclic. In these cases, the local strain rate is calculated using simplified relations, derived from the HRR-field solution for a stationary crack [83]. In case of constant load and at temperatures where the creep is not a concern, the crack tip strain rate will consist only of the convective term. The convective term, however, is not sufficient to keep the crack advance and the crack will arrest in a limited period of time. This observation was made by finite element calculations [90], and supported by a number of recent experimental reports [89, 91-94]. It is emphasized in all these studies, that an active load application is a vital prerequisite for continuous crack growth. In case of constant load, the cracks observed there only developed within a short time interval after the load application. Note that although the crack tip is thought as a linear segment in the film rupture mechanism, the crack kinematics is actually a straight motion of a point. An alternative view [90] considers the crack profile as a smooth surface, which evolves in time due to corrosion. 


\section{EXPERIMENTAL SYSTEM}

The experimental system (see Figure 35) consisted of the following three major components: (1) a supercritical water loop with two test reactors, (2) a low-temperature loop, and (3) a data acquisition system. The system was designed for parallel study of the kinetic parameters of the oxide films and SCC in preloaded specimens at supercritical temperatures. This is the only system capable of providing in situ information on oxide film behavior at supercritical temperatures. Another unique feature that measured information on oxide films was synchronized with experimental data on crack propagation under the same conditions. The system has been in operation for two years. During this period in situ measurements have been performed continuously using automatic data collection.

\section{CDE Arrangement for Supercritical Water}

A CDE arrangement for in-situ studies of the oxide films at supercritical temperatures was designed and built during the first year (Figure 19). The arrangement is installed inside a guiding tube attached to the reactor vessel head. Axial movement of the upper specimen is controlled by a moving rod equipped with a micrometer. Accuracy of the vertical positioning of the CDE specimens is within a fraction of a micron. Specimens are electrically insulated from the holders, the guiding tube, and the reactor vessel (Figure 20). The samples were constructed as two parallel surfaces (tips of small diameter rods) as shown in Figure 21. A special fitting with four connectors (Figure 22a) is used for connection of the CDE specimens and electrodes to ex-vessel equipment. Connectors inside the vessel are insulated using ceramics flexible insulators (Figure 22b).

The CER measurements performed during the first year are based on bringing the surfaces of two specimens into contact under an accurate displacement control and measuring contact resistance at the working surfaces. An HP4338A milliohmmeter was used for four probe method resistance measurements. This system makes it possible to determine in situ changes in the resistance of the protective layers on the surfaces from resistance vs. displacement measurements (Figure 23). The specimens were made of the candidate structural materials (in this case SS304, SS316, and SS347). 


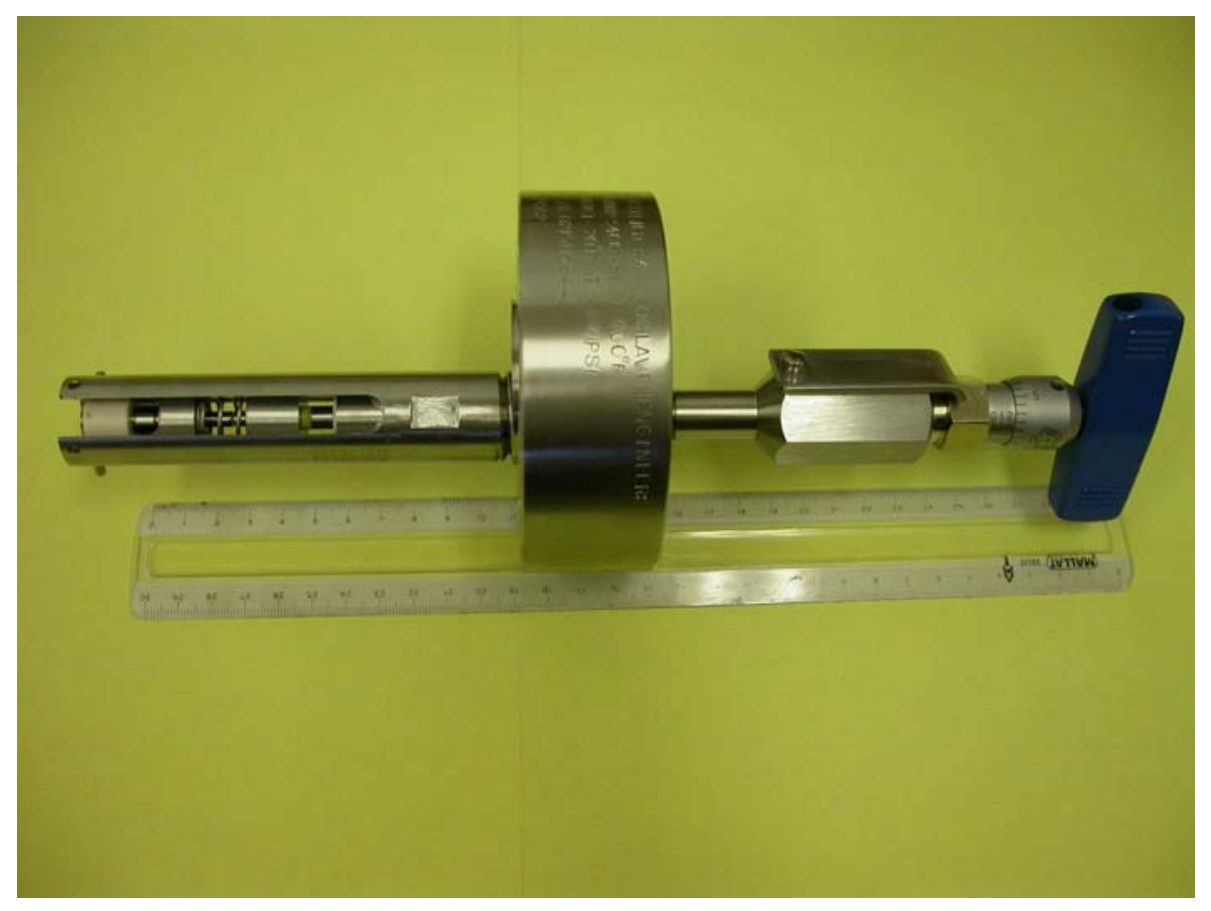

(a)

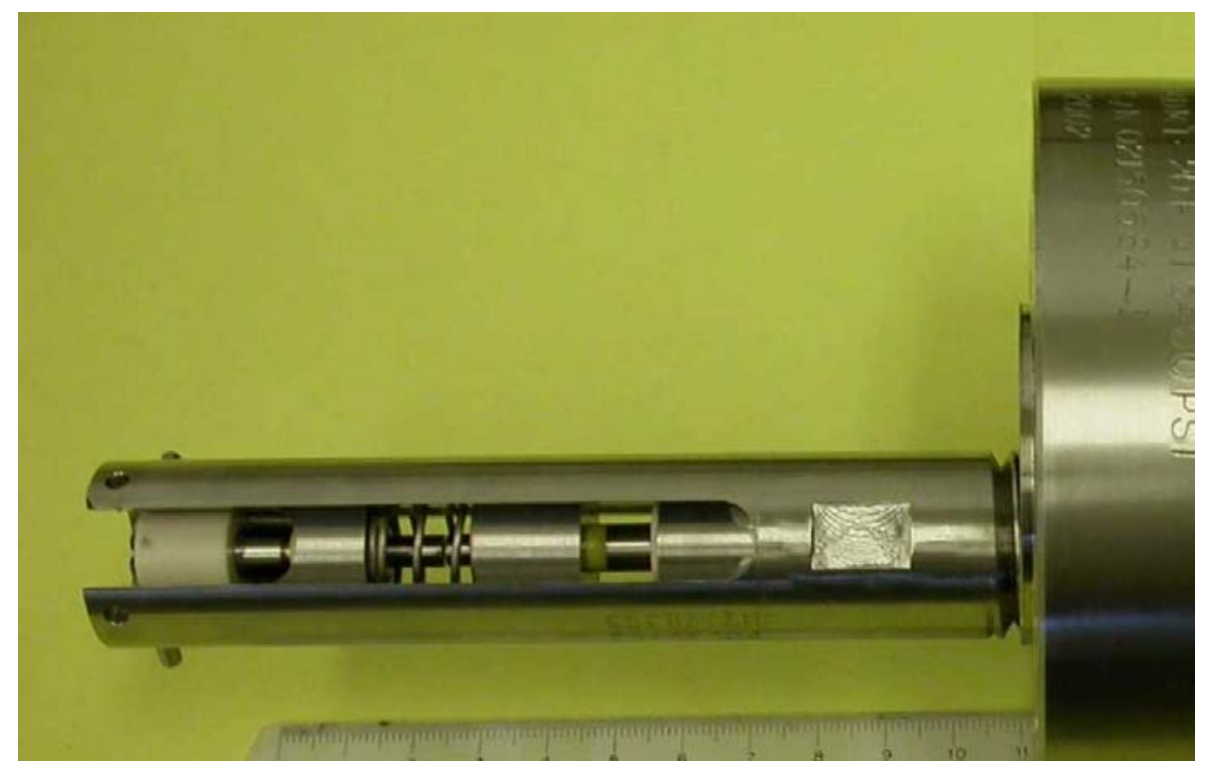

(b)

Figure 19. Arrangement for controlled distance electrochemistry studies at supercritical temperatures. (a) general view and (b) close view showing guiding tube, specimen holders, and CDE specimens. 


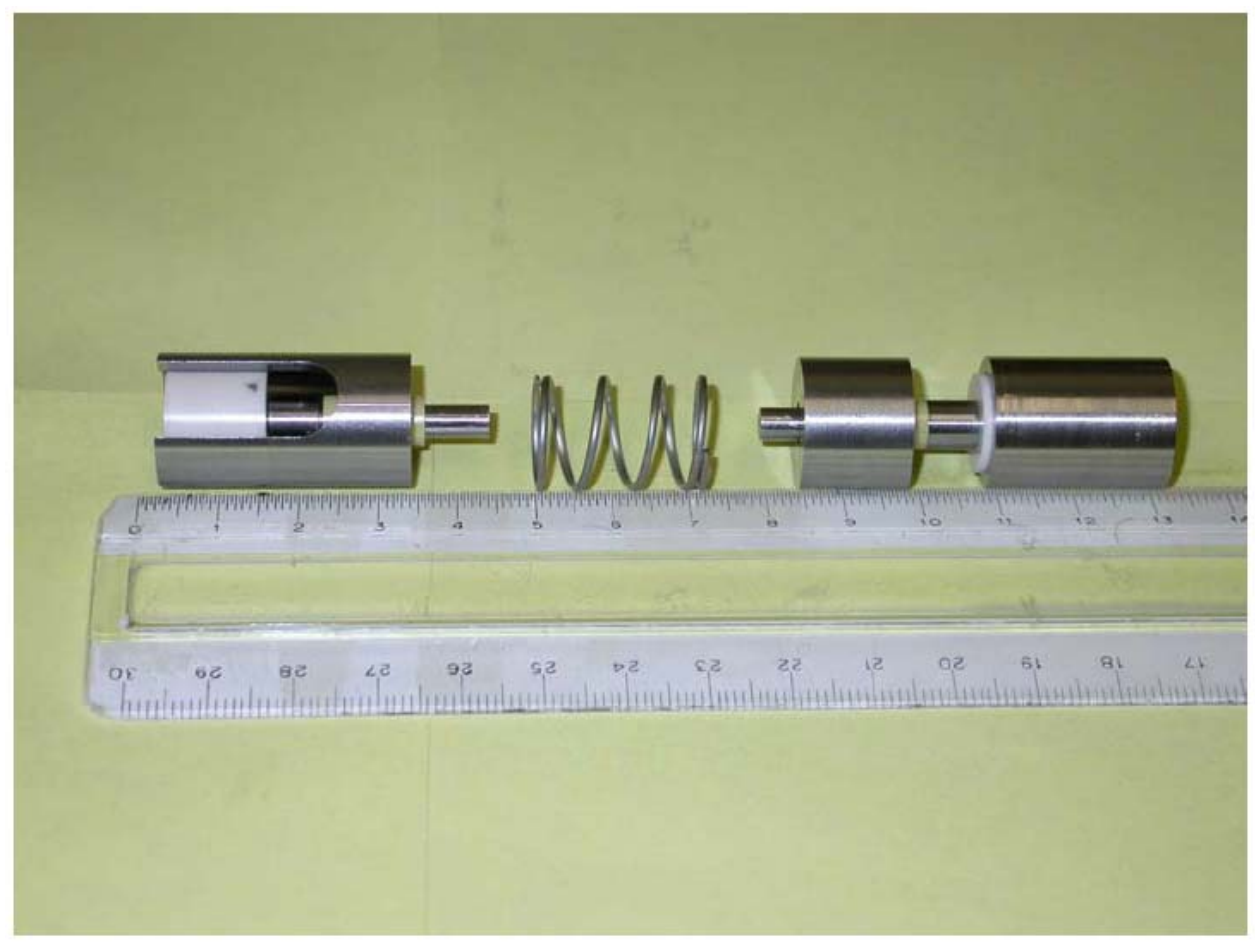

Figure 20. Details of CDE arrangement.

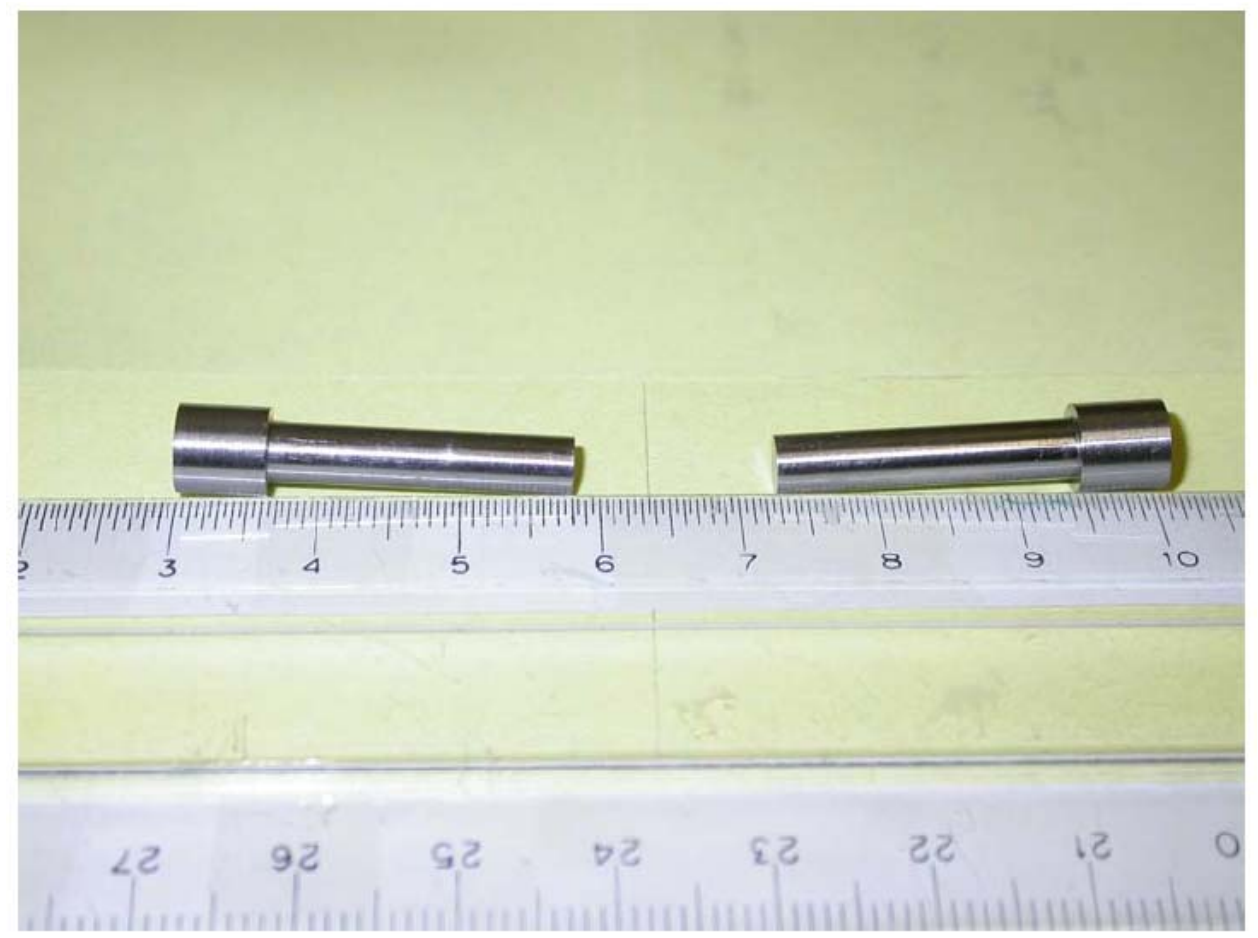

Figure 21. CDE specimens. 


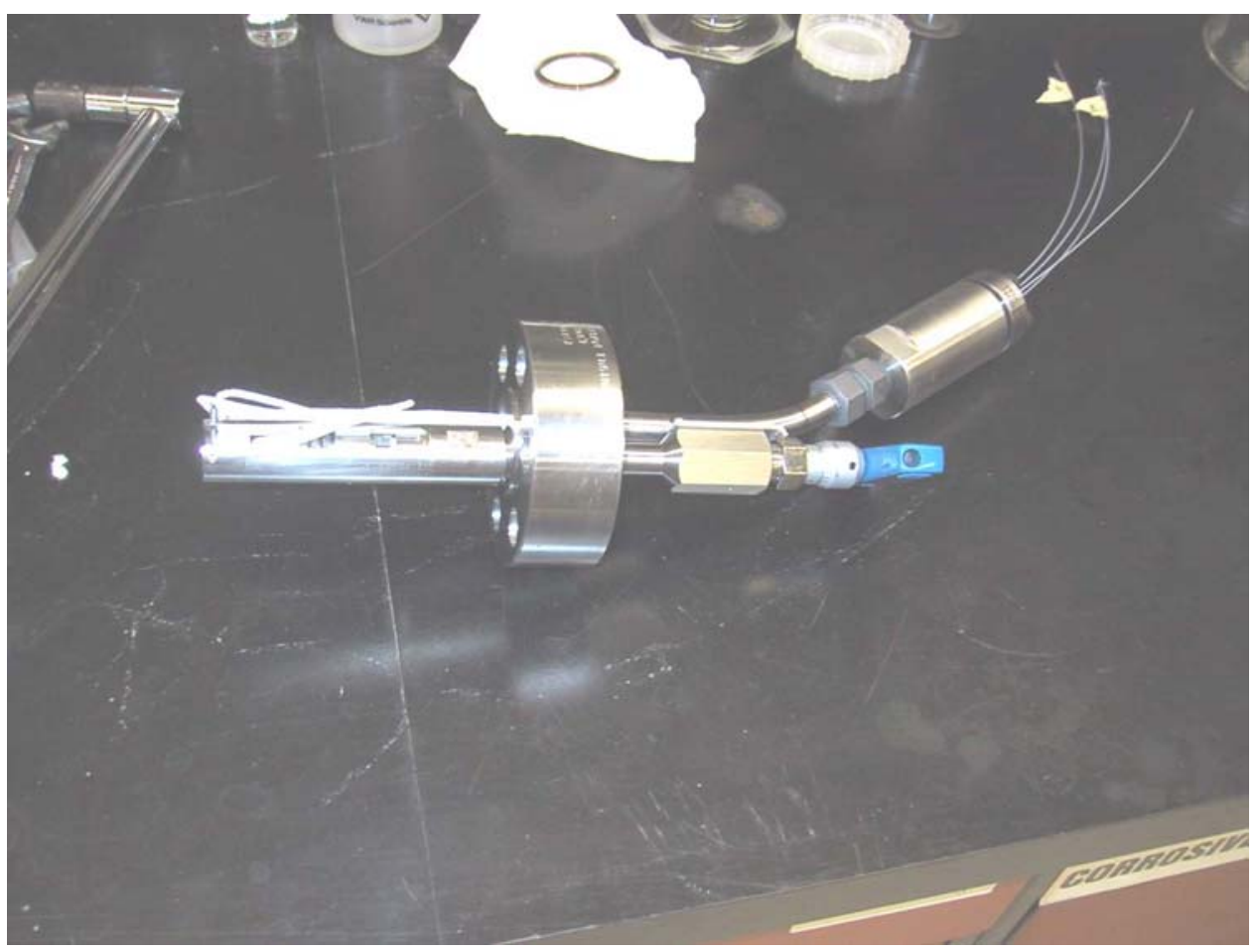

(a)

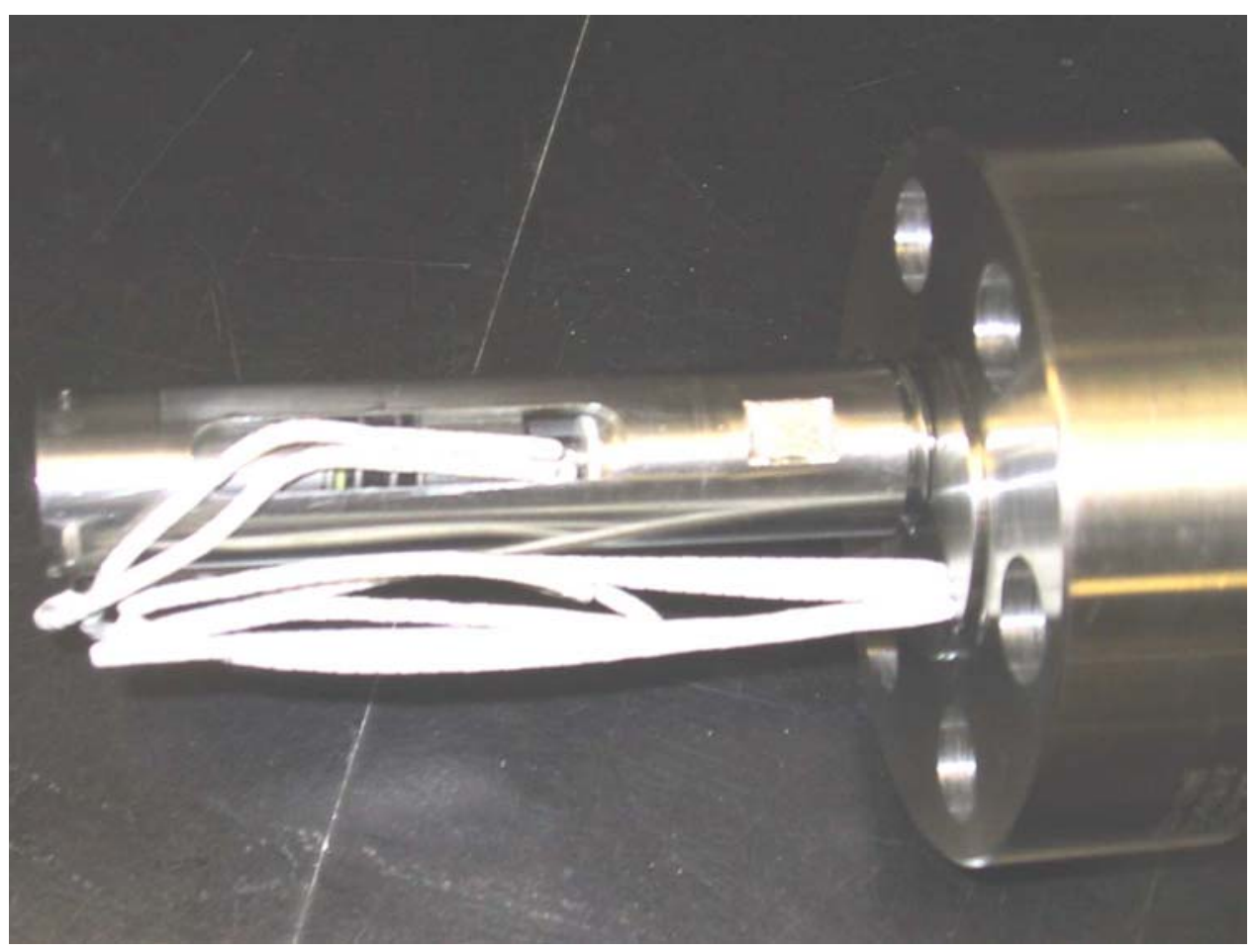

(b)

Figure 22. Electrical connection of the CDE specimens. 


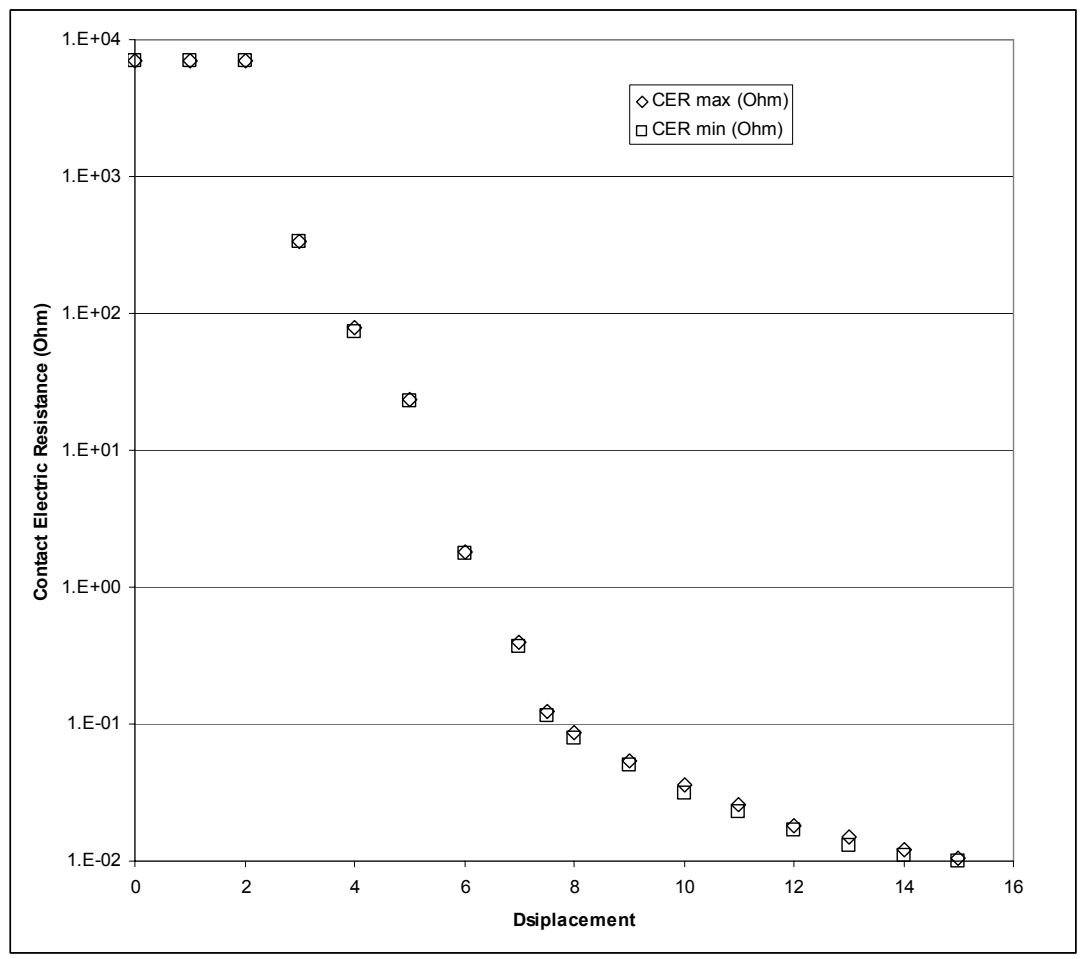

Figure 23. Variation of the contact electric resistance with distance between working surface of CDE specimens.

\section{Specimens for SCC Testing}

The SCC specimens were going to be tested in an autoclave at and above supercritical water conditions. It is very difficult to attach and use any externally controlled loading devices that go through the pressure boundaries at supercritical temperatures. Furthermore, working with high pressure and high temperature, there is a severe size requirement on the autoclave and on the specimen dimensions. For this reason we decided to choose a specimen and loading system that can be compact and stand-alone.

Another important consideration was the difficulty in initiating and propagating a crack in SCC mode from a fatigue pre-cracked tip that was introduced at room temperature and loaded prior to the exposure to the environment. One possible technique to counteract this difficulty is to assist active crack growth in the environment by creating a rising stress field. Thus, the desirable property of the specimen is a rising stress intensity field at the beginning followed by decreasing stress intensity field under a given initial loading condition.

Based on the above considerations we chose to explore a centrally notched circular disc specimen with a pins-and-wedge loading system as shown in Figure 24. A finite element analysis 
was used to evaluate the stress intensity variation as a function of crack length under prescribed loading conditions.

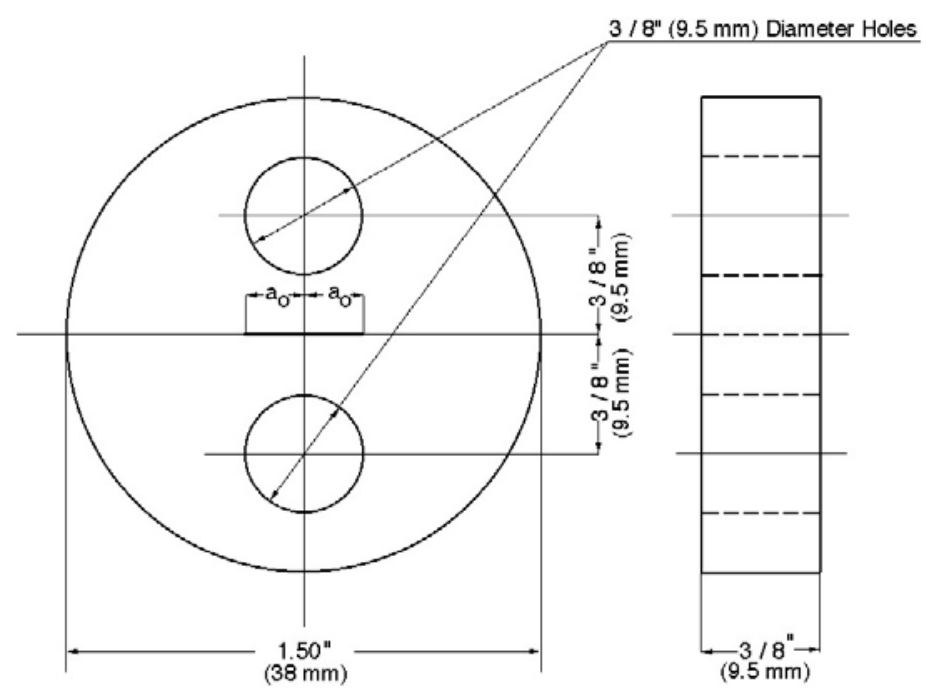

(a) Disc Specimen Geometry
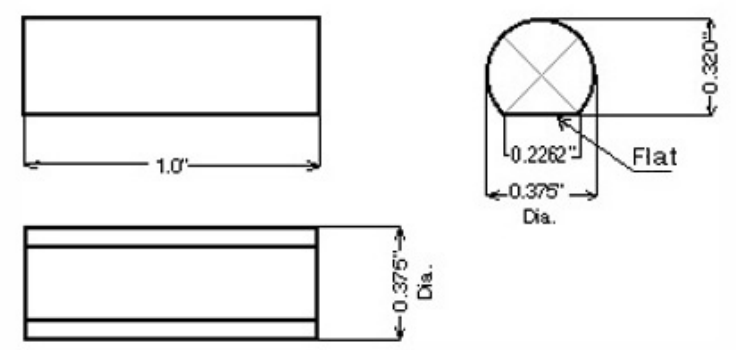

Dia.

(b) Loading Pin
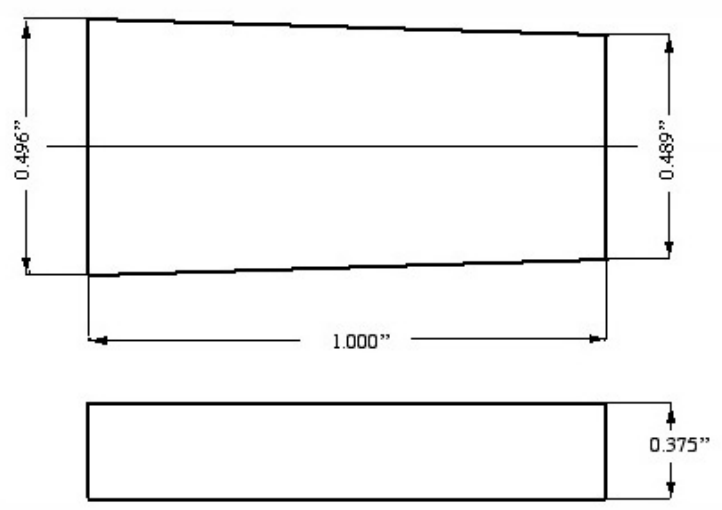

(c) Loading Wedge

Figure 24. Specimen geometry and loading pins and wedges. 
Finite element analysis of the stress intensity variation was performed using the FRANC 2DL code developed by Cornell University and modified by University of Kansas. Figure 25 shows the mesh in the disc specimen and the loading pins. The stress intensity at the crack tip was calculated by specifying a fixed displacement at the flat face of one of the pins while the position of the flat face of the other pin was fixed. We calculated a series of stress intensities by changing the crack length while maintaining the fixed displacement. The computation was repeated for several different displacements. Figure 26 shows the stress distribution inside the disc at a chosen crack tip position.

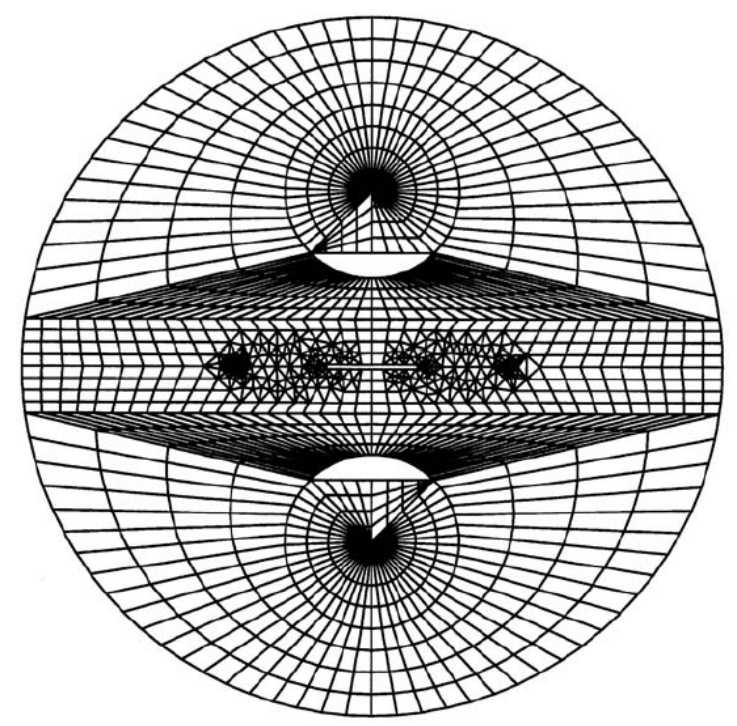

Figure 25 Mesh used in the finite element analysis of the center notched disc.

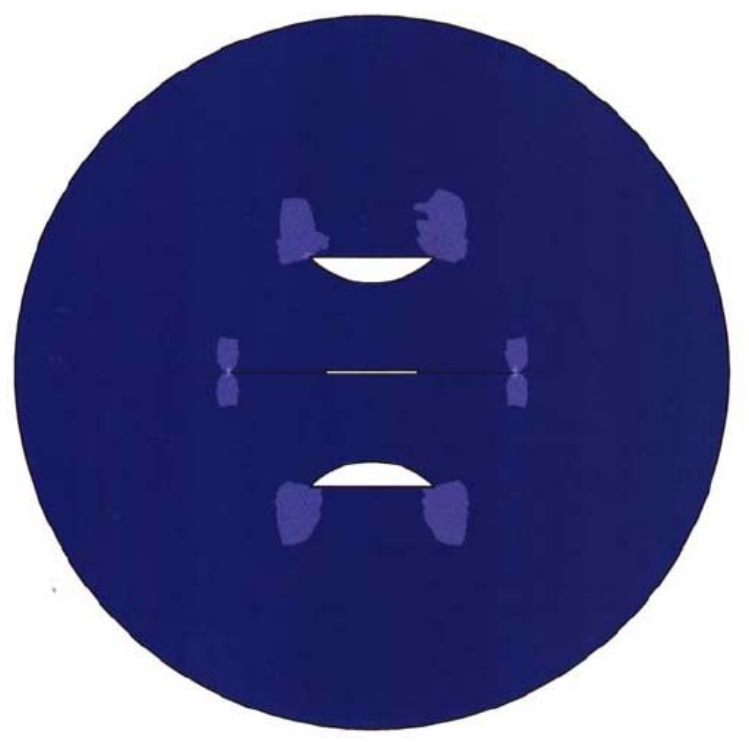

Figure 26. Effective stress distribution in the center notch disc under fixed pin-displacement loading. 
Figure 27 shows the stress intensity factor variation as a function of crack length under a unit fixed displacement $(0.001 \mathrm{inch} / 0.025 \mathrm{~mm}$ displacement $)$. With this specimen geometry, it is clear that stress intensity initially increases monotonically, reaches maximum at the crack length of 0.2 inches, and decreases linearly up to the crack length of 0.4 inches. Beyond the crack length of 0.4 inches, the stress intensity reduction rate slows, but continues to decrease.

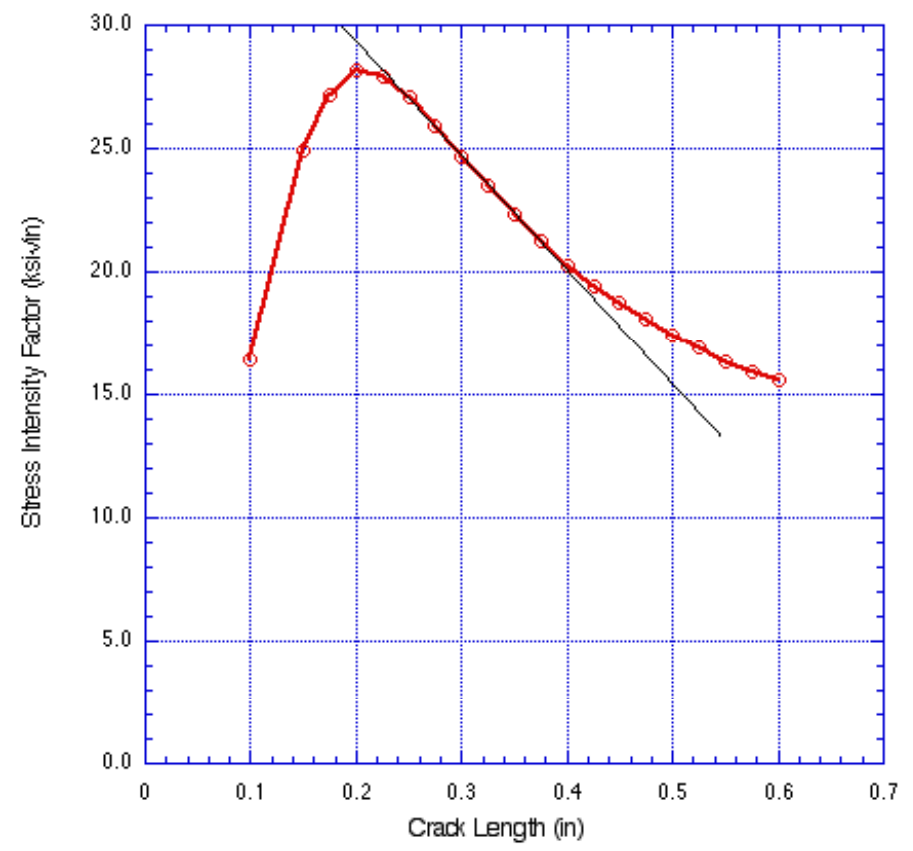

Figure 27. Variation of stress intensity as a function of crack length under a unit fixed displacement.

The stress intensity field under fixed displacement loading condition appeared to be ideally suited for stress corrosion experiment in extreme environments. We could induce a fatigue pre-crack of length less than 0.2 inches, load the crack to a desired stress intensity level, and expose it to the environment. The initial rising stress intensity field could assist initiation and growth of the crack in the environment. The frequently encountered problem of difficulties in transforming the fracture mode of the transgranular mechanism during fatigue pre-cracking into the intergranular mechanism could possibly be solved by assisting the crack growth in the environment for some distance by increasing stress intensity as the crack extends. The crack in the environment will eventually slow down and stop due to the decreasing stress intensity field.

Ten specimens were fabricated with an electron beam produced center notch of length 0.20 inches $(\mathrm{a}=0.10$ inches $)$. We attempted to initiate fatigue pre-crack from the notch; 
however, as the curve in Figure 27 indicated, the stress intensity level at a $=0.10$ inch was too low to initiate a fatigue crack within a reasonable time.

We attempted to increase the displacement amplitude or load amplitude, but the general yielding around the pin holes occurred. For this reason we decided to modify the fabricated specimen by cutting one side open by electron beam cutting (Figure 28).
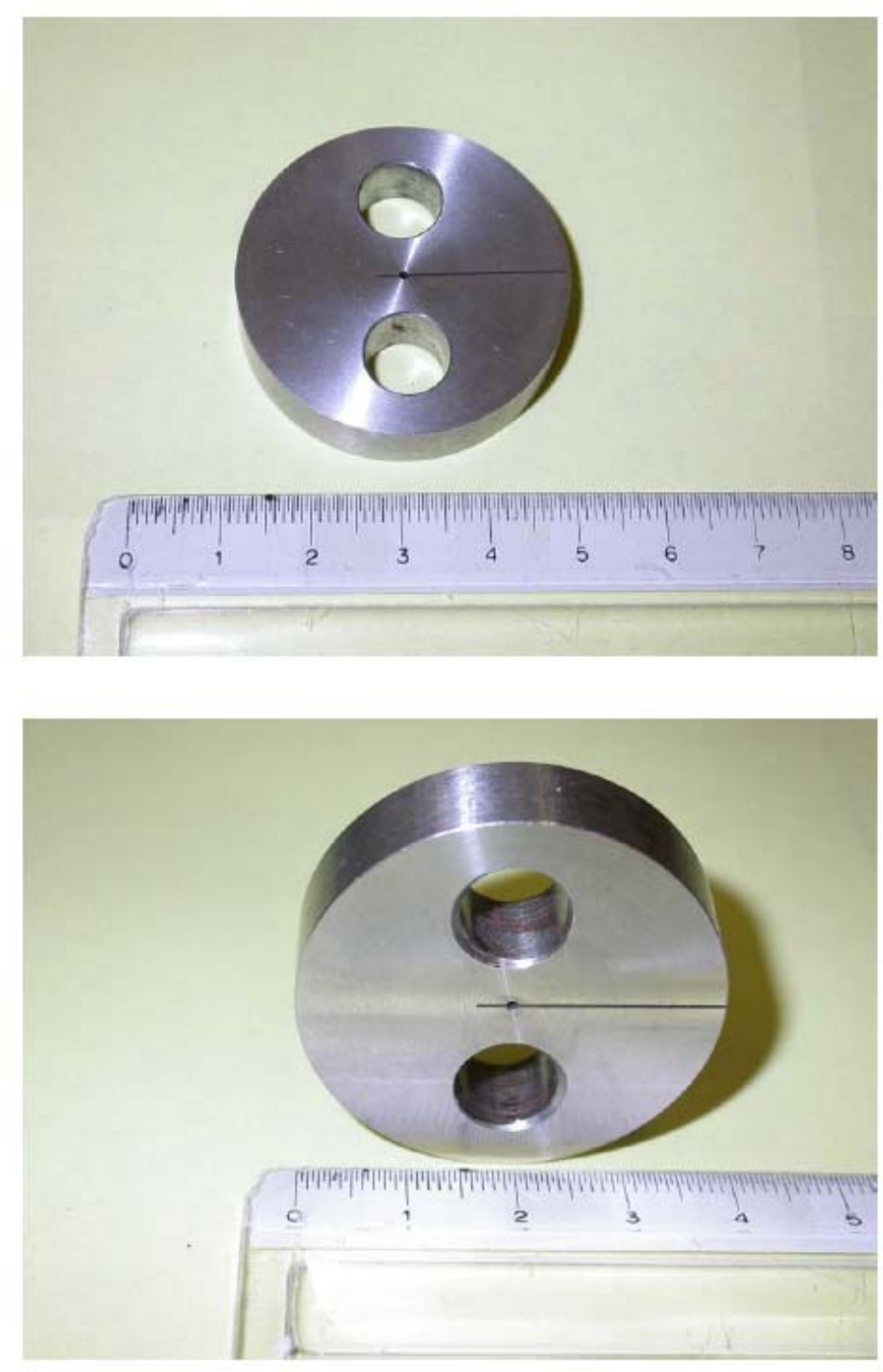

Figure 28. Single edge notch disc specimen

Figure 29a shows the finite element mesh used in the analysis of single edge notch disc specimen at the crack tip position at $\mathrm{a}=0.30 \mathrm{inch}$. Figure $29 \mathrm{~b}$ shows the magnified deformation of the disc, and Figure 30 shows the effective stress distribution. We see a distinctive butterfly type stress distribution associated with a crack tip. 


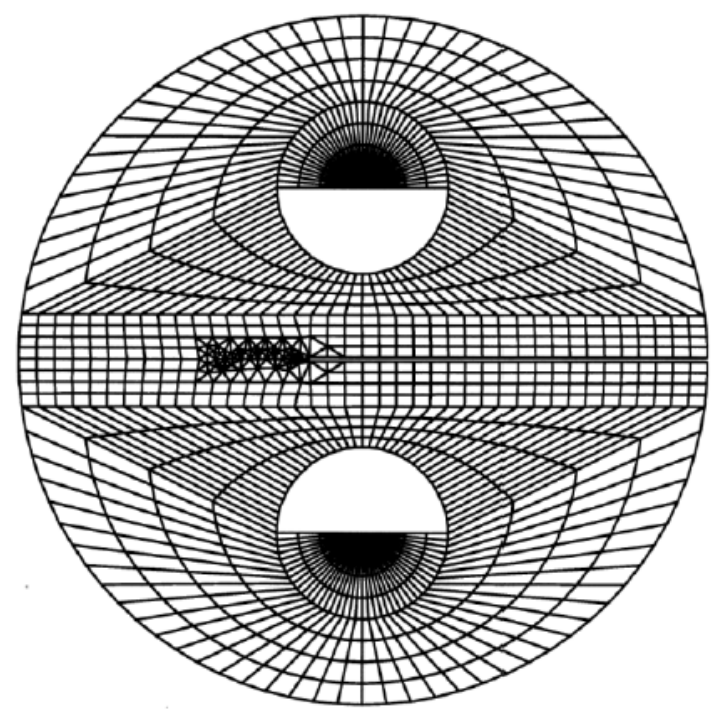

(a)

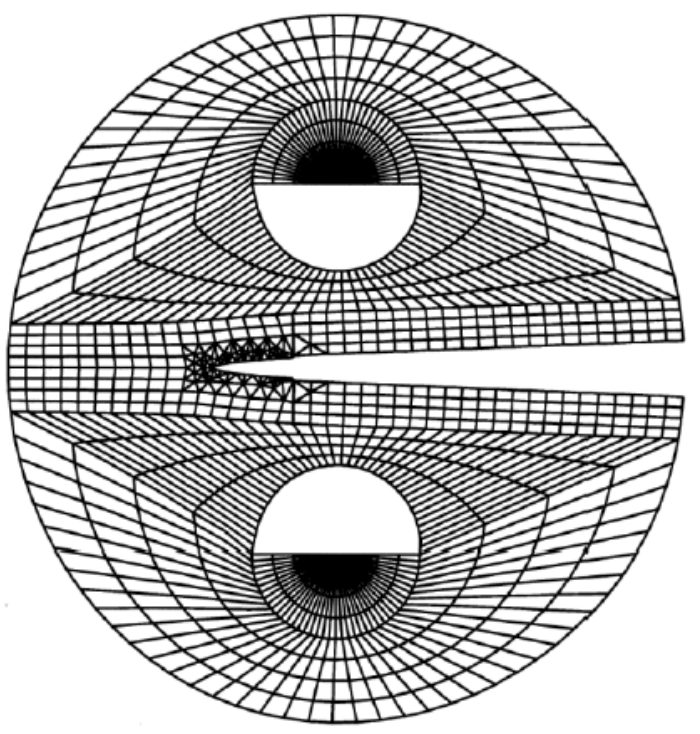

(b)

Figure 29. Meshes used in the finite element analysis of the single edge notched disc and deformed geometry of the disc under fixed pin displacement of 0.005 inches. The crack length was 0.30 inches. (a) finite element meshes in the disc; (b) deformed geometry under fixed pin displacement of 0.005 inches.

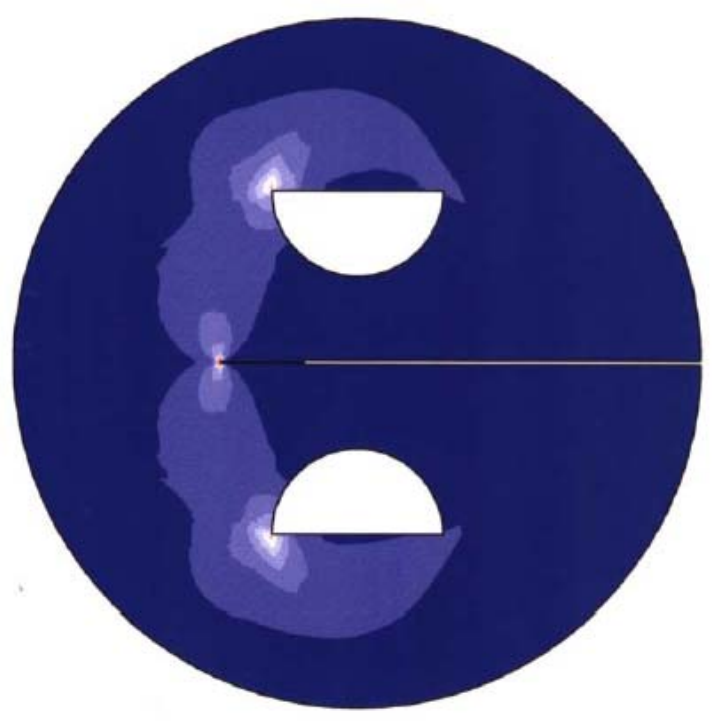

Figure 30. Effective stress distribution in the disc under fixed pin displacement of 0.005 inches at the crack length $\mathrm{a}=0.30$ inches. 
It is noted that the stress concentration around the half pins are due to the simple model used for ease of analysis, and that in reality the stress concentration is not as severe as indicated here. The stress intensity variation as a function of crack length under various fixed pindisplacement setups was calculated and shown in Figure 31.

The oscillation of the stress intensity when the crack length is short is caused by meshing effects in the finite element analysis and is not a real physical phenomenon. The variation of the stress intensity as a function of crack length in the single-edge-notch disc specimen is monotonic decrease. However, for a given displacement we could produce higher stress intensity than that in the center-notch disc specimen.

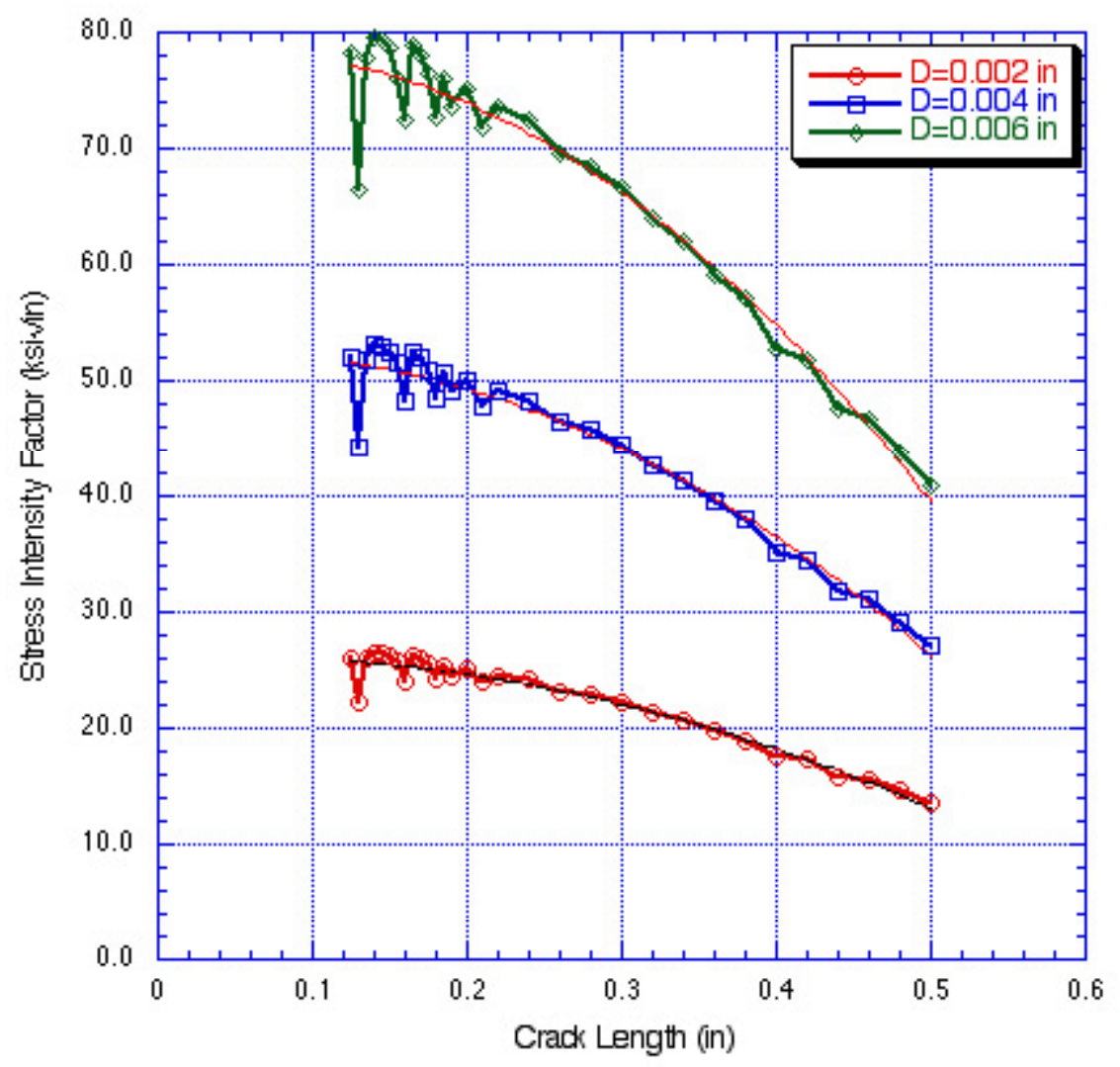

Figure 31. Stress intensity factor variation as a function of crack length under fixed pin displacement loading condition. Three cases of pin-displacement are shown. 
Fatigue pre-cracking was done in MTS servo-hydraulic testing machine. Figure 32 shows an experimental setup. The load-controlled constant amplitude fatigue loading with $\mathrm{R}=$ 0.05 at frequency of $20 \mathrm{~Hz}$ was used initially to induce quickly a fatigue crack at a tip of the electron beam notch. The maximum load between 1500 pounds and 2000 pounds was used.

During the fatigue cycling, the pin displacement was observed using two Zimmer electrooptical displacement transducers, Model 100D. This transducer has a frequency response of $400 \mathrm{kHz}$ and a resolution of $\pm 1 \mu \mathrm{m}$. As the fatigue crack was formed at the notch tip, the displacement shift was observed. After the displacement shift was observed, the loading condition was changed either to reduce the maximum load or to switch to displacementcontrolled mode so that the maximum stress intensity was reduced and the crack grew at a slower rate.

The fatigue pre-cracking process was stopped when the crack extension of about 0.075 inches occurred. The specimen was removed from the loading machine and photographs of the specimen surfaces were made to record the crack tip position. Figure 33 shows an example of the crack initiated at the electron beam notched tip.

The specimen was then loaded with the pins and wedges shown in Figure 24. By adjusting how deep to insert the wedges between the pins, we can control the stress intensity at the crack tip. For the initial experiment, we have inserted the wedges to the maximum depth so that the pin-displacement of 0.006 inches was accomplished. Figure 34 shows a photograph of a wedge-loaded single-edge-notch disc specimen before exposure to supercritical water. 


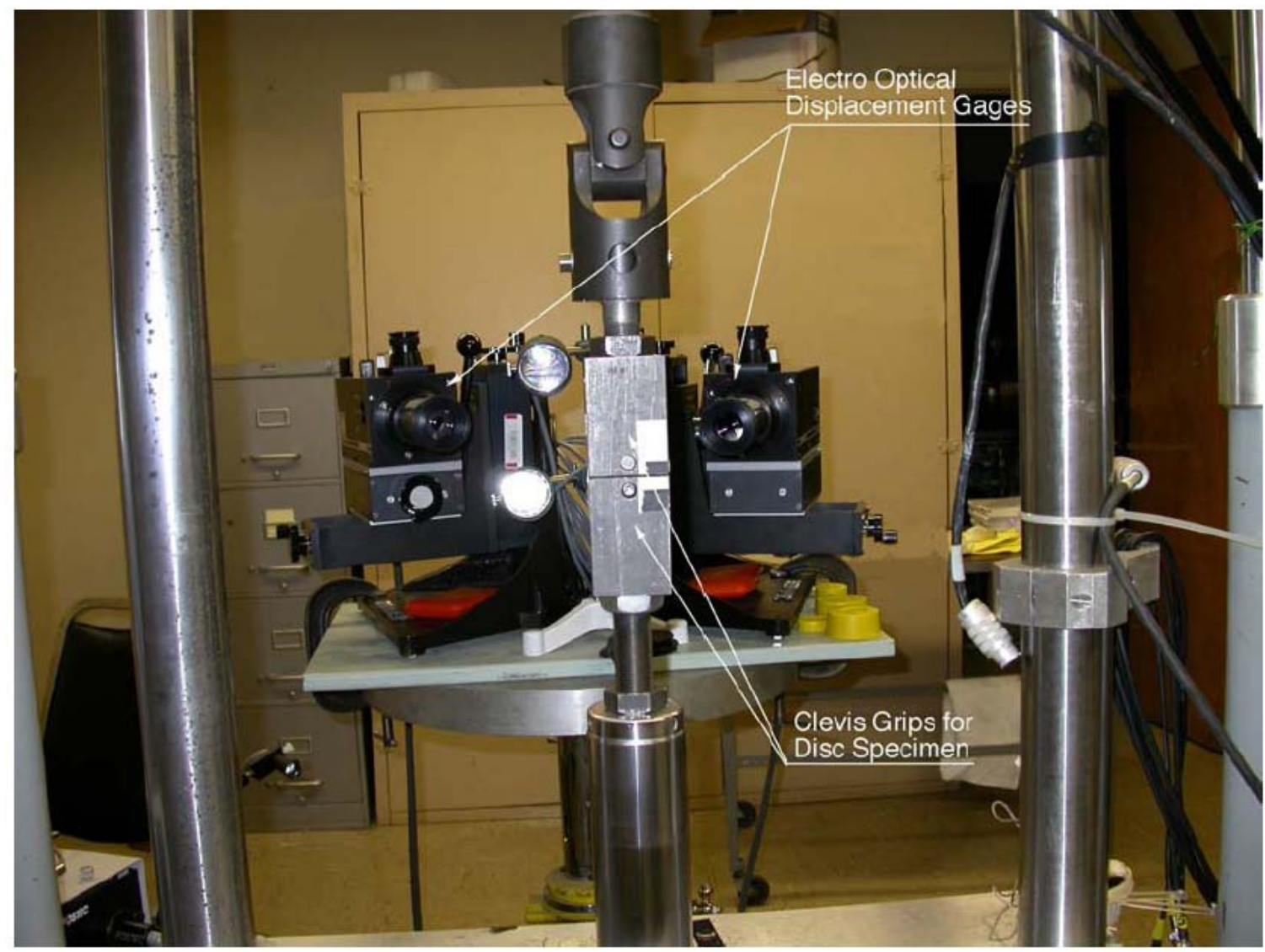

Figure 32. Experimental setup showing two electro-optical displacement transducers.
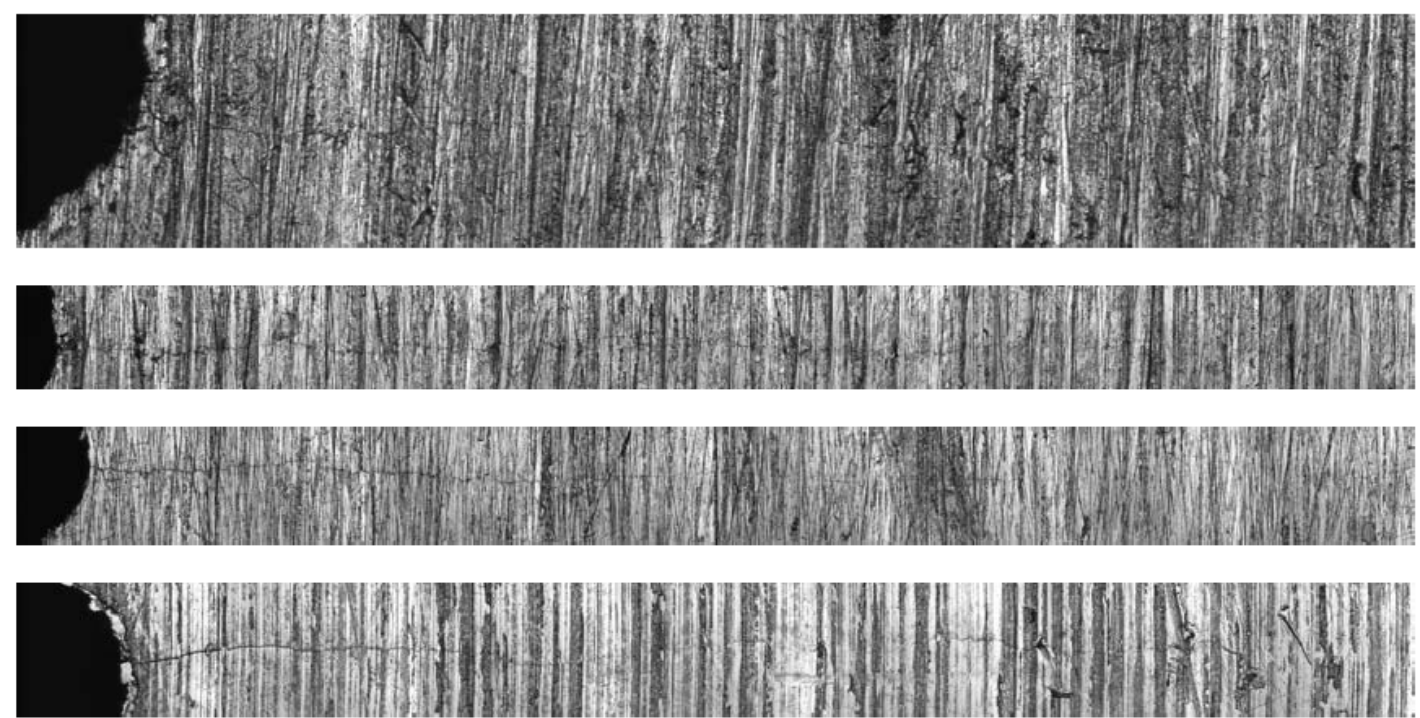

Figure 33. Macro photograph of the side of the disc specimen showing the fatigue precrack extending from the electron beam notched tip. 

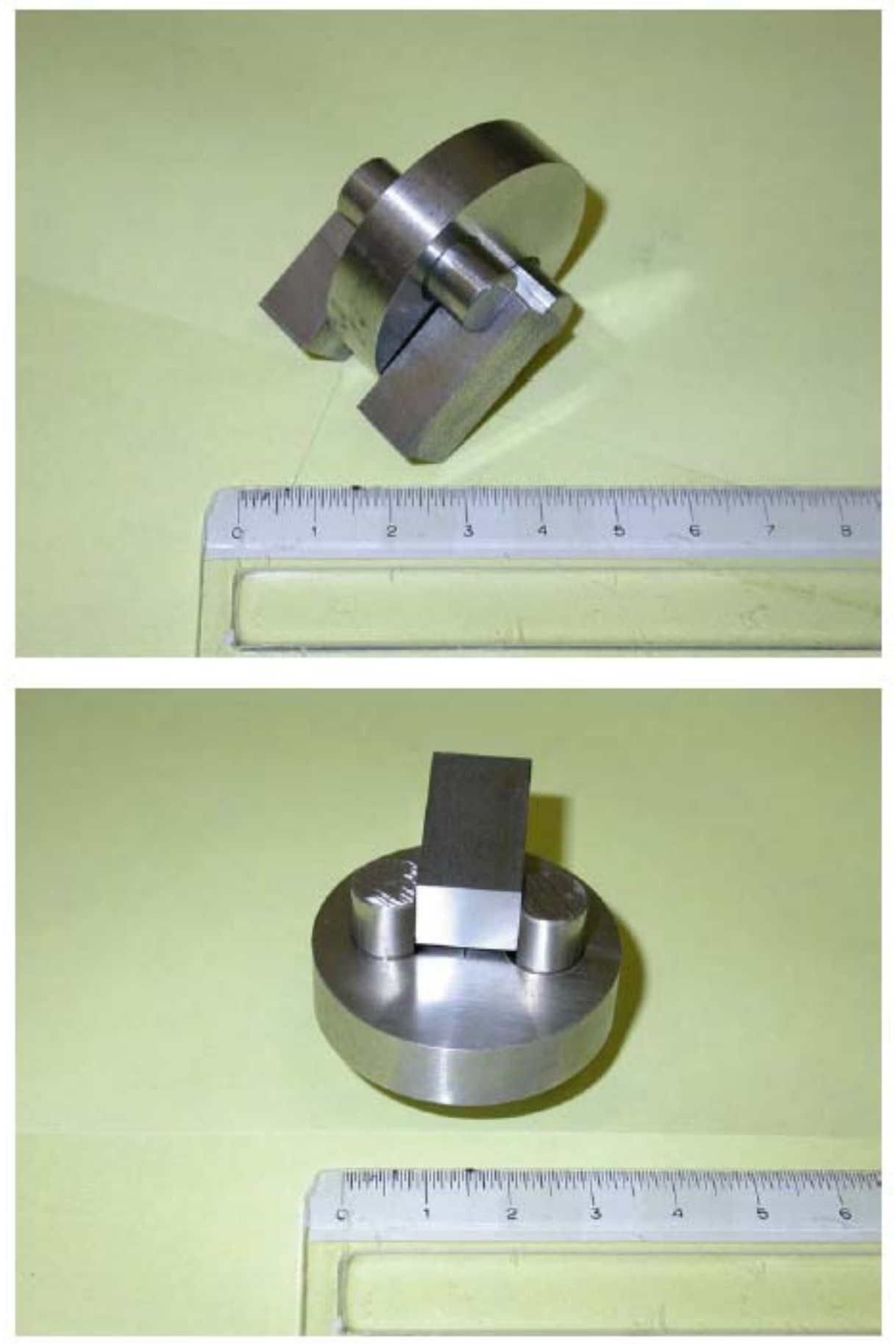

Figure 34. Wedge loaded single-edge-notch disc specimen ready to be placed in the autoclave. 


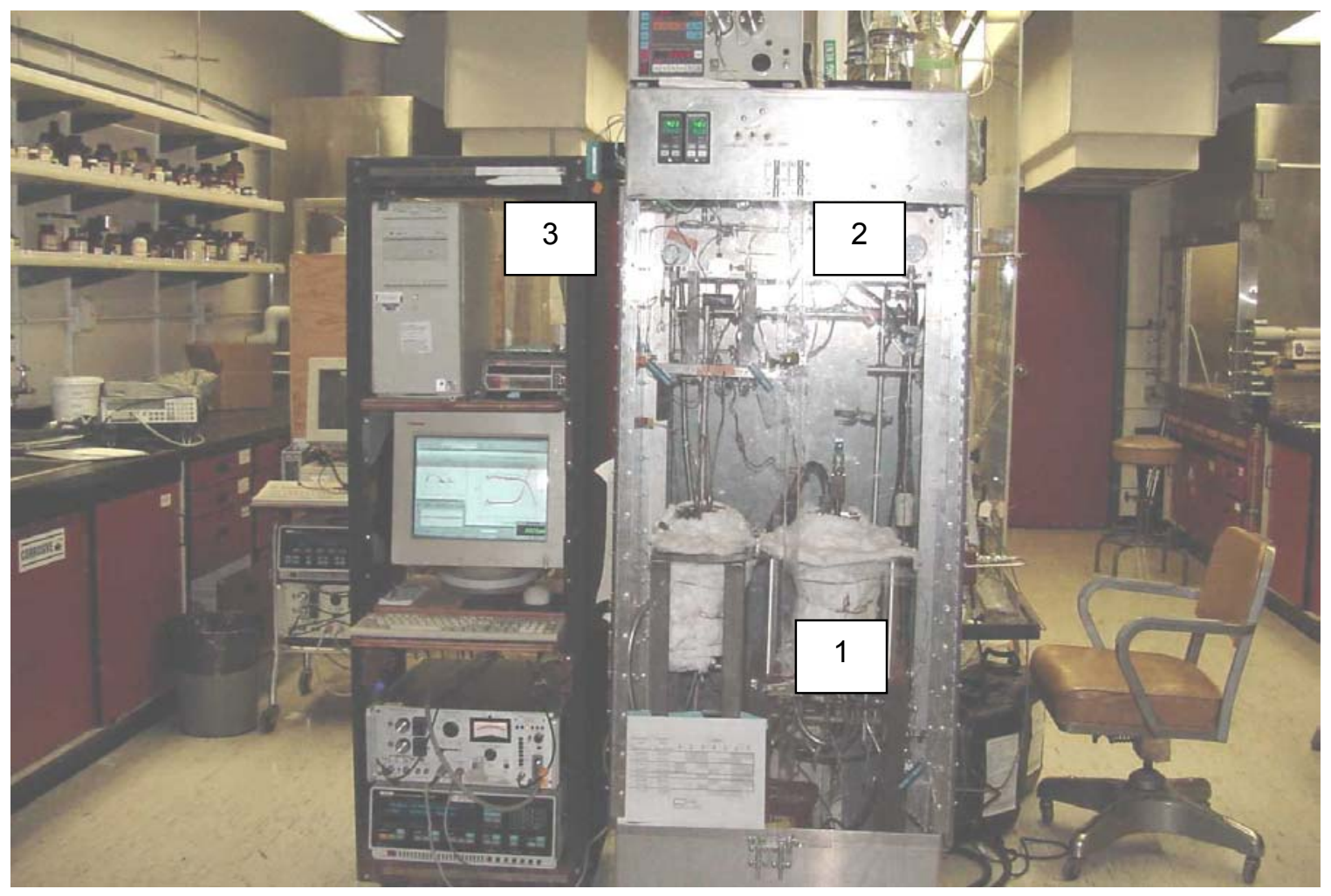

Figure 35. General view of the experimental system: supercritical test reactors (1), the highpressure loop (2), and the data acquisition system (3).

\section{Supercritical Water Loop}

CDE and SCC studies are being performed in two test reactors installed in the supercritical water loop (see Figure 36). Exposure of the CDE specimens and in situ measurement of kinetic processes in the oxide films are being performed in the CDE reactor. FM prestressed specimens are being exposed in the autoclave for prestressed specimens (APSS) reactor. Both reactors can be operated independently in three modes: (1) flow through, (2) static, and (3) shutdown for replacement of specimens or maintenance (see Table 2). Figure 37 provides a close view of the test reactors. As many as four fracture mechanics specimens and two CDE specimens can be studied at a time.

The system operated at temperatures from $400^{\circ} \mathrm{C}$ to $450^{\circ} \mathrm{C}$ and at pressures up to 5,000 psi. A computer-controlled emergency shutdown system is activated when pressure or temperature deviates from a preset range. 


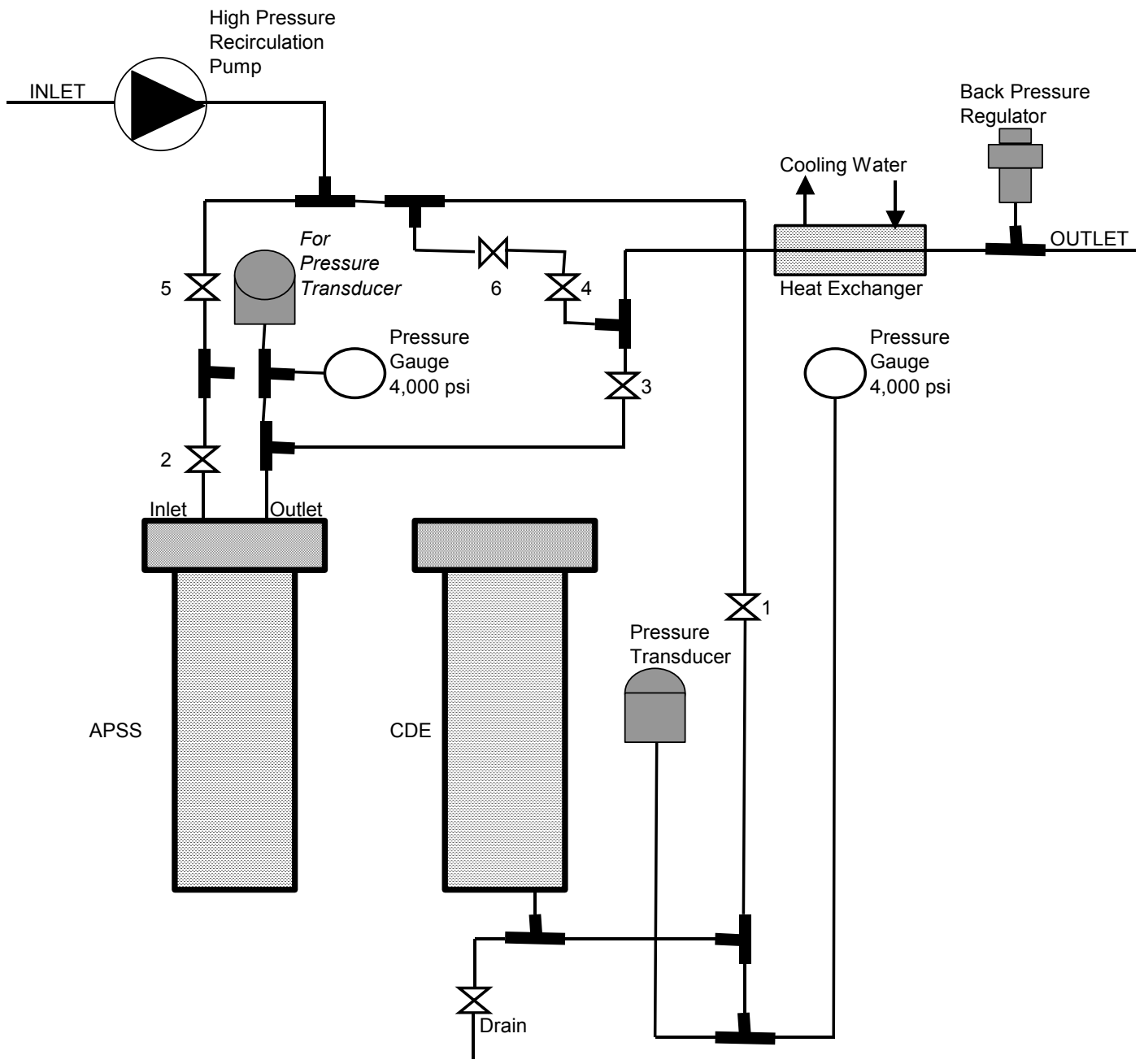

Figure 36. Diagram of the supercritical water loop and test reactors.

Table 2. Operation modes of the test reactors in the supercritical water loop.

\section{Reactor 1 Reactor $2 \quad$ Valves}

$\begin{array}{ccccccccc}\text { SCC } & \text { CDE } & 1 & 2 & 3 & 4 & 5 & 6 & 7 \\ \text { Specimens } & \text { Specimens } & 1 & 2 & 3 & & \end{array}$

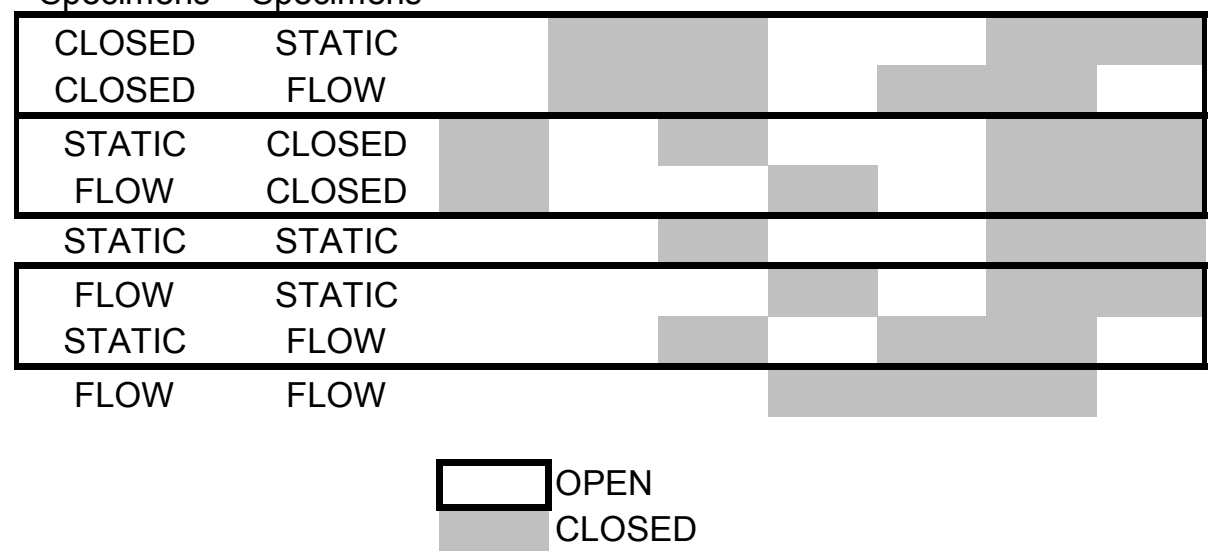




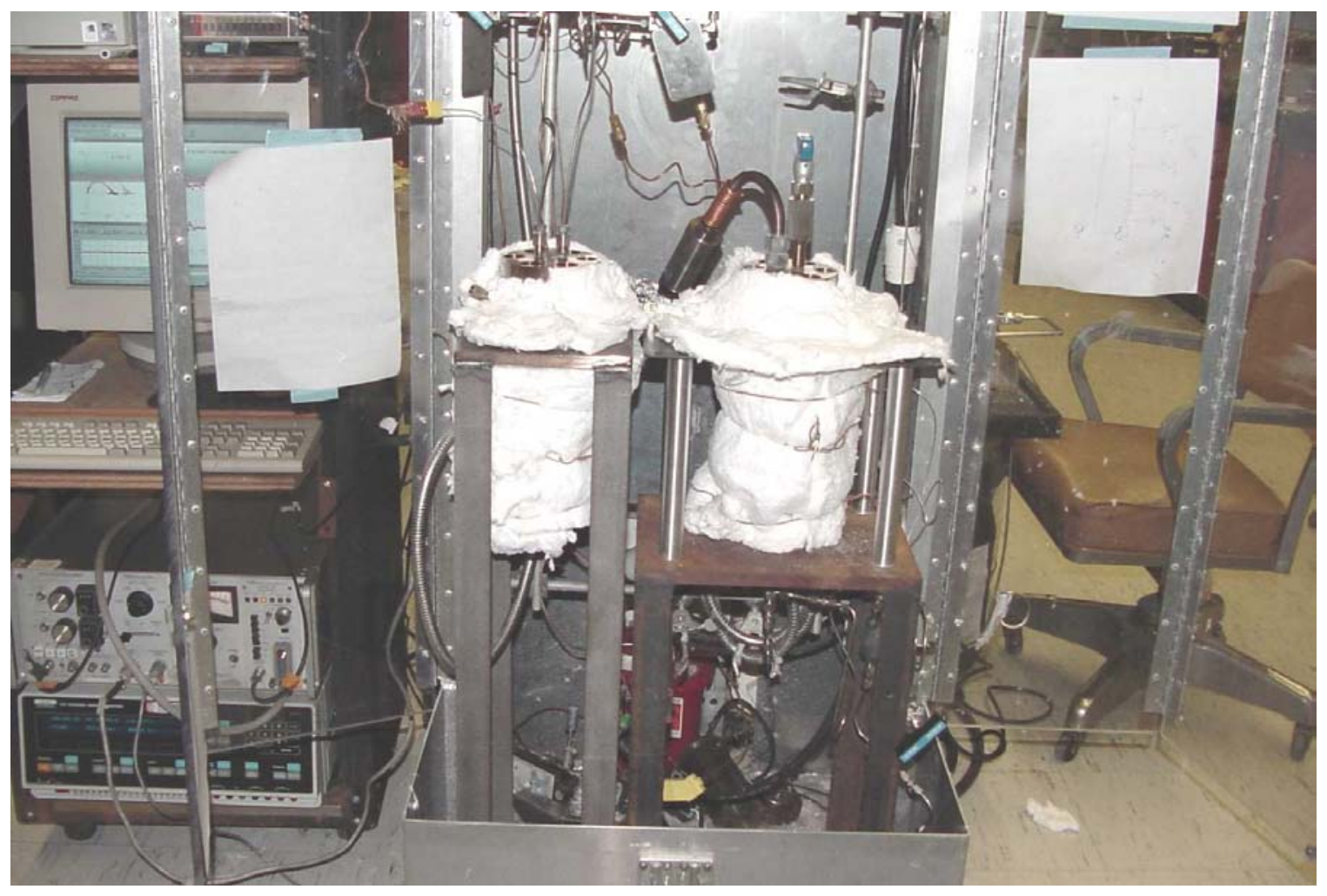

Figure 37. Close view of the APSS reactor (left) and CDE reactor (right)

\section{Low-Temperature Loop}

Solution chemistry in the test reactors (conductivity and concentration of dissolved oxygen) is controlled by a low-temperature loop (see Figure 38). Purified water from the refill tank is pumped into a stainless steel mixing tank. The desired level of oxygen is maintained by bubbling He gas through a solution in the mixing tank. Pressure in the low-temperature loop is maintained at $\sim 30$ psi. Oxygen and conductivity sensors can be switched to monitor the inlet or outlet flow of the test reactors using valves 11-13 (shown in Figure 38). The oxygen sensor, the conductivity cell, and flow level sensors are connected to the data acquisition system. 


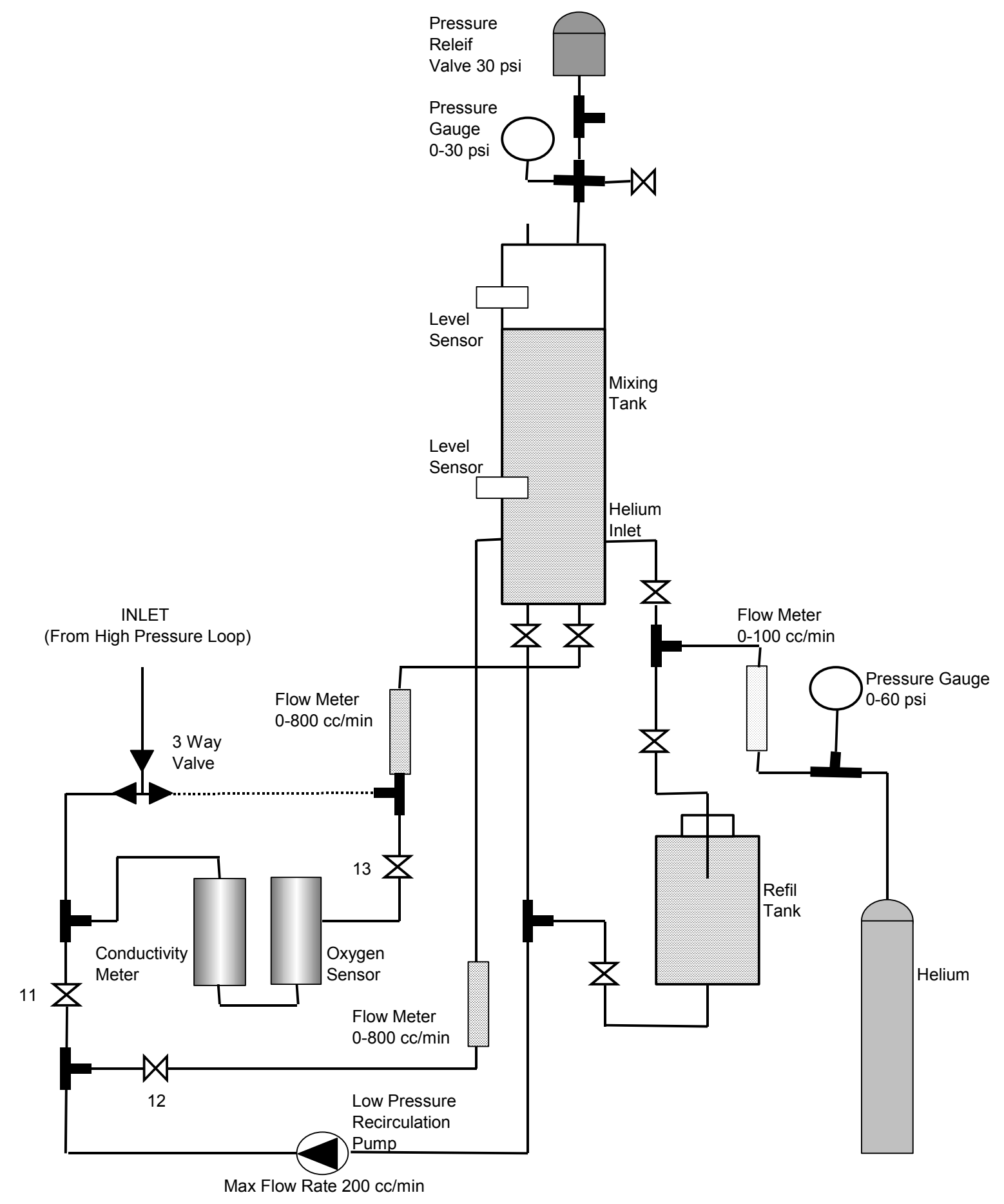

Figure 38. Diagram of the low-temperature loop. 


\section{CDE Measurements}

Pretreatment of the CDE specimens consisted of polishing with emery paper up to grade 4000 , rinsing with ultrapure water (resistivity $>18 \mathrm{M} \Omega \mathrm{cm}$ ), and drying with hot air. Specimens were positioned $2 \mathrm{~mm}$ apart during the heating of the reactor. TLEC measurements were performed with a gap between samples ranging from $10 \mu \mathrm{m}$ to $2 \mathrm{~mm}$. Most of the measurements were performed in an automatic regime using a batch mode of Zplot data acquisition software, with intervals ranging from 1 to $12 \mathrm{~h}$. CEI measurements were performed when specimens were in contact. The contact position was defined from CER measurements at the position where resistivity drops from several kohms to several ohms. CEI measurements were performed in manual mode after it was found that keeping the specimens in contact during CEI measurements for a prolonged time altered the oxide film.

\section{Impedance Measurement System}

Electrochemical impedance spectra were measured with a Solartron 1250 system (see Figure 39) controlled by / ZPlot software (Scribner Associates) in a frequency range of 0.001 to $65,000 \mathrm{~Hz}$ at an AC amplitude of $20 \mathrm{mV}$ to $400 \mathrm{mV}$ (rms). The impedance spectra was validated by checking the linearity condition; that is, by measuring spectra at signal amplitudes between 20 and $400 \mathrm{mV}$ (rms), and by checking the causality using a Kramers-Kronig (K-K) compatibility test based on Boukamp's procedure [68].

ZPlot with its data display/analysis companion program ZView (see Figure 40) was selected because it is the most flexible and powerful impedance software available. A distinctive feature of Zplot/Zview is that it was developed by electrochemists rather than by electrical

engineers. As a result, data acquisition and processing are focused on the physical meaning of the results. The wide selection of the "physical-model"-based circuit elements was found to be particularly helpful in the current project. Use of Zplot/Zview also minimized routine work on preliminary analysis and processing of hundreds of impedance spectra measured during the reported period. Use of batch file to define a sequence of impedance measurements run measurements at prescribed time allowed 24-hour data collection. 


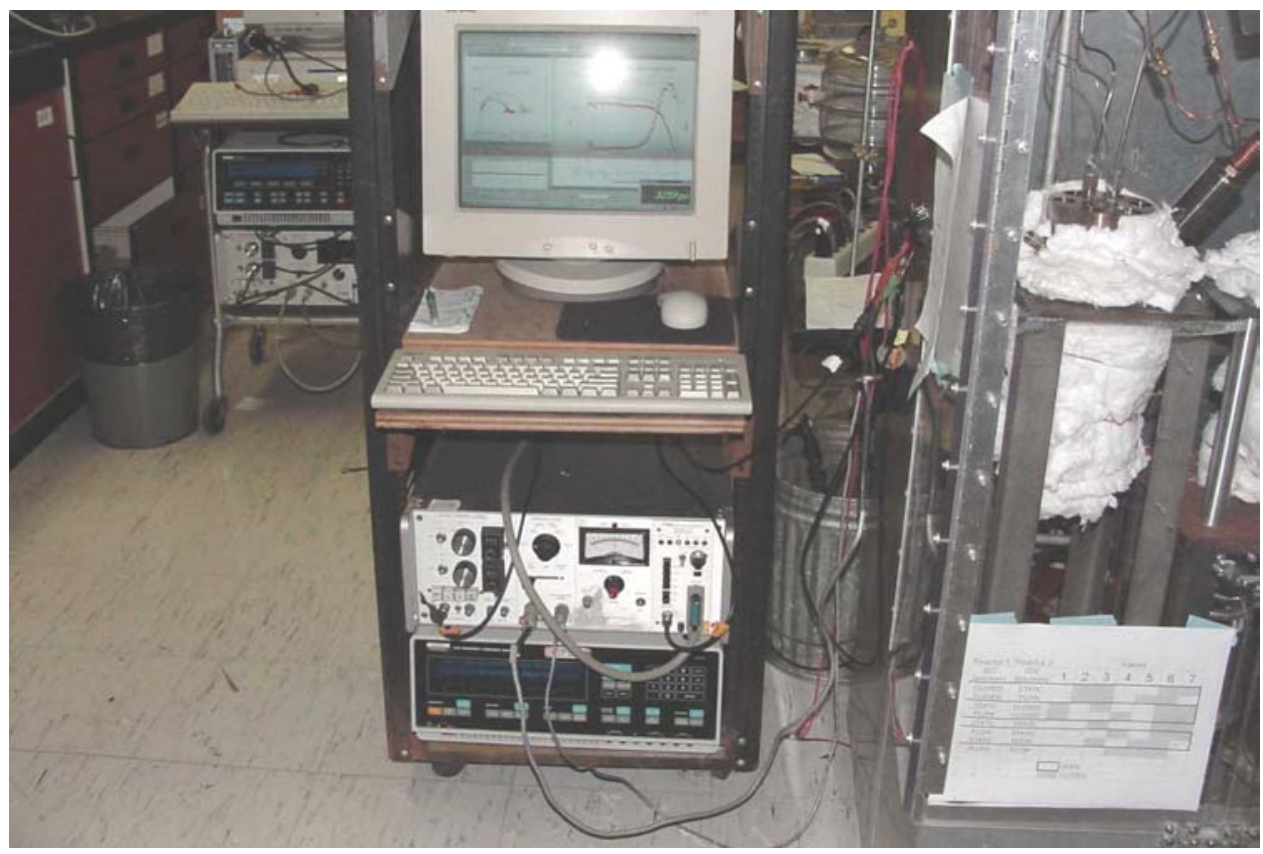

Figure 39. Modules of the impedance measurement system.

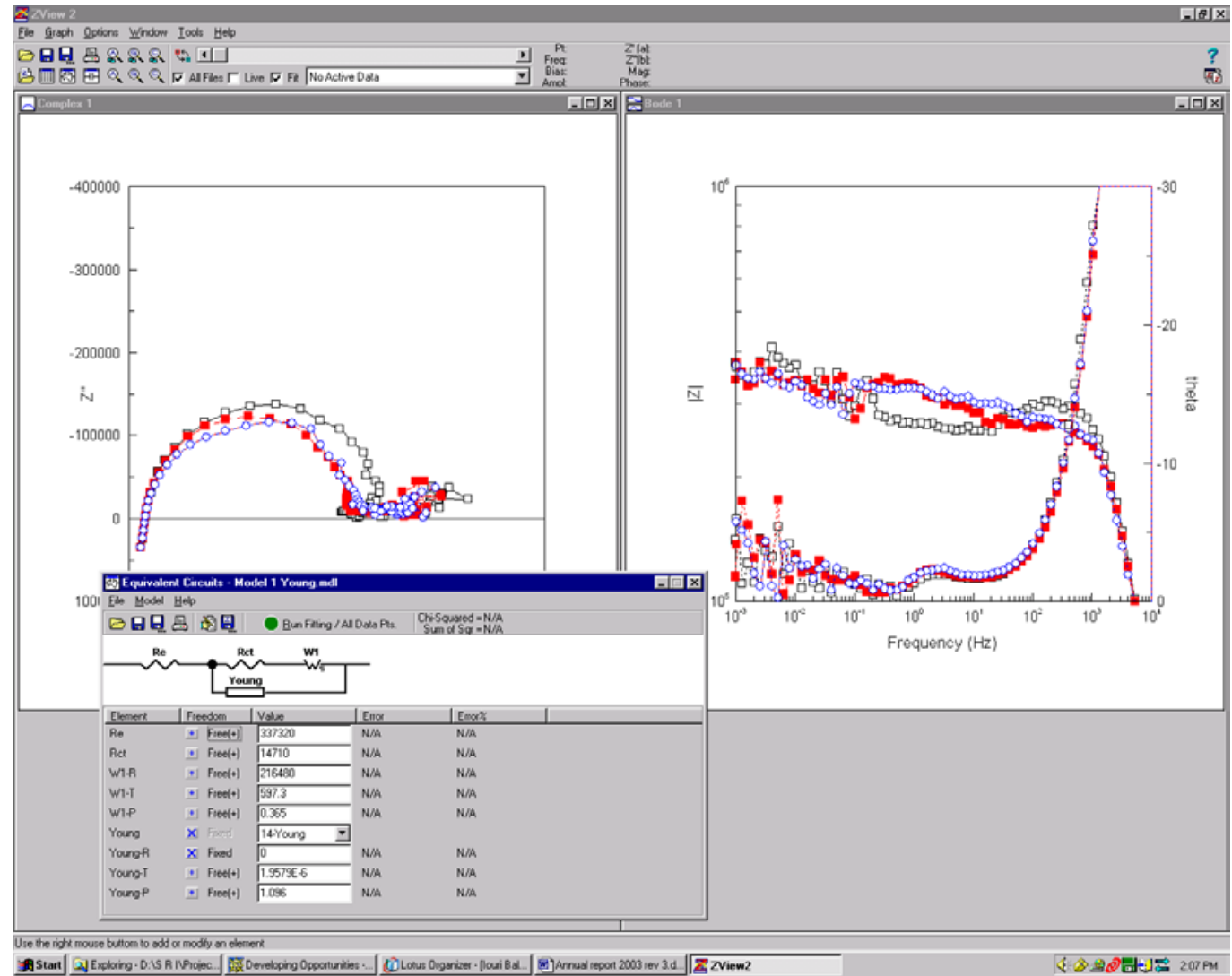

Figure 40. Display of the impedance measurement and analysis system. 


\section{Data Acquisition System}

The data acquisition system consisted of data acquisition hardware (DaqBoard 2000 and DBK202 by Iotech) and data acquisition software (DasyLab/LE supplied by Iotech). DaqBoard200 is a 16-bit, 200-kHz analog-to-digital (A/D) converter with 8 differential or 16 single-ended analog inputs (software selectable per channel) expandable up to 256 analog input channels, while maintaining a $200-\mathrm{kHz}$ scan rate. Each channel is configurable for voltage, current, temperature, and various other signals directly or via a signal conditioner. DBK is an adapter panel with screw terminals, which also connects signal conditioning modules, if necessary.

Measured data were digitized by an A/D converter (DaqBoard 2000) and forwarded to DasyLab. Experimental configuration and processing blocks were programmed using DasyLab language (see Figure 41). The input stream of data was processed on the fly (averaging, statistical analysis), displayed, and stored on a hard drive by DasyLab data acquisition software.

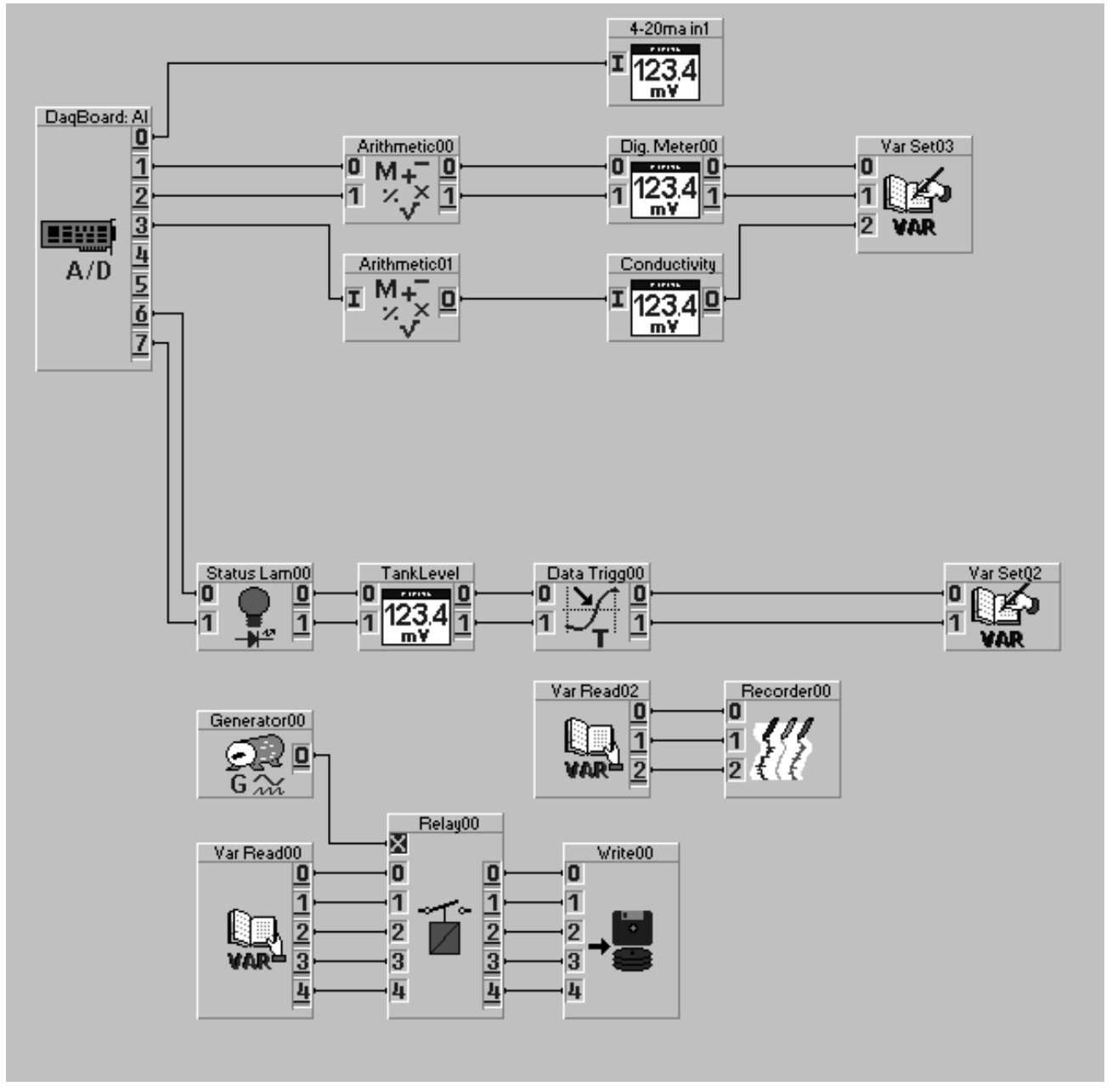

Figure 41. Modules of the data acquisition system. 


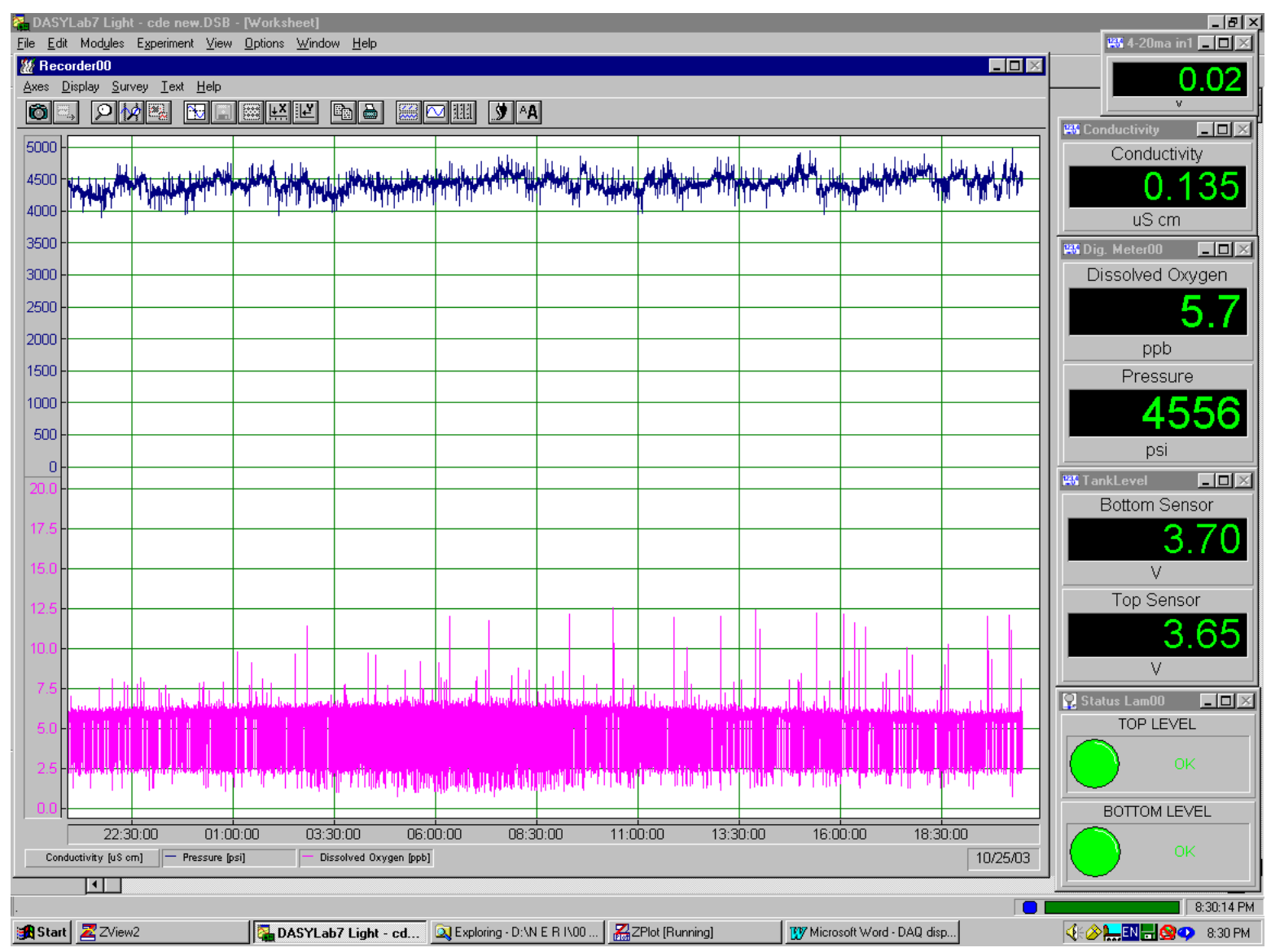

Figure 42. Display of the data acquisition system

\section{EXPERIMENTAL CONDITIONS}

Electrochemical and fracture studies that were performed in parallel during the reporting period took place in two separate test reactors at a temperature of $400^{\circ} \mathrm{C}$ and a pressure of 3500 psi. Water chemistry conditions were similar for all tests: a high level of dissolved oxygen $(\sim 4$ $\mathrm{ppm})$ and low conductivity $(\sim 0.3 \mu \mathrm{S} / \mathrm{cm}$ at room temperature). All three candidate austenitic steels-SS304, SS316, and SS347-were exposed to the highly oxidizing conditions to rank these materials according to their susceptibility to general and localized corrosion in the supercritical water. The behavior of the best candidate alloy from each material group (austenitic, ferritic-martensitic, and nickel-based alloys presented in Table 3) will be measured during the third year of the project under oxidizing conditions ranging from 5-10 ppb to $1 \mathrm{ppm}$ of dissolved oxygen concentrations. 
Table 3. Chemical composition of candidate and tested materials.

\begin{tabular}{|c|c|c|c|c|c|c|c|c|c|c|c|}
\hline Comment & & $\mathrm{Fe}$ & $\mathrm{Cr}$ & $\mathrm{Ni}$ & $\mathrm{Mn}$ & $\mathrm{C}$ & Si & $\mathrm{P}$ & $\mathrm{S}$ & Mo & $\mathrm{Nb}$ \\
\hline Reference & 304 & Balance & 18.0-20.0 & $8-10.5$ & 2 & 0.08 & 1 & 0.045 & 0.03 & - & \\
\hline Reference & TP 304 & Balance & $18.0-20.0$ & $\begin{array}{l}8.0- \\
11.0\end{array}$ & 2 & 0.08 & 0.75 & 0.04 & 0.03 & - & \\
\hline Reference & $304 \mathrm{H}$ & Balance & $18.0-20.0$ & $\begin{array}{l}8.0- \\
12.0\end{array}$ & 2 & $0.04-0.10$ & 1 & 0.045 & 0.03 & - & \\
\hline Reference & TP $304 \mathrm{~L}$ & Balance & $18.0-20.0$ & $\begin{array}{l}8.0- \\
11.0\end{array}$ & 2 & 0.035 & 0.75 & 0.04 & 0.03 & - & \\
\hline Heat 407568 & 304 & Balance & 18.05 & 8.64 & 1.84 & 0.028 & 0.53 & 0.027 & 0.002 & 0.43 & \\
\hline Reference & TP 316 & Balance & $16.0-18.0$ & $\begin{array}{l}11.0- \\
14.0\end{array}$ & 2 & 0.08 & 0.75 & 0.04 & 0.03 & $2.0-3.0$ & \\
\hline Reference & TP $316 \mathrm{~L}$ & Balance & $16.0-18.0$ & $\begin{array}{l}11.0- \\
14.0\end{array}$ & 2 & 0.035 & 0.75 & 0.04 & 0.03 & $2.0-3.0$ & \\
\hline Heat $A 070836$ & 316 & Balance & 16.1 & 10.11 & 1.65 & 0.012 & 0.58 & 0.029 & 0.023 & 2.09 & \\
\hline Heat 231584 & 316 & Balance & 17 & 11.2 & 1.57 & 0.015 & 0.38 & 0.028 & 0.001 & 2.06 & \\
\hline Reference & TP 321 & Balance & $17.0-20.0$ & $\begin{array}{l}9.0- \\
13.0\end{array}$ & 2 & 0.08 & 0.75 & 0.04 & 0.03 & - & \\
\hline Reference & 347 & Balance & $17.0-19.0$ & $\begin{array}{l}9.0- \\
12.0\end{array}$ & 2 & 0.08 & 1 & 0.04 & 0.03 & 0.75 & \\
\hline Heat 232212 & 347 & Balance & 17.45 & 9.15 & 1.45 & 0.04 & 0.52 & 0.031 & 0.022 & 0.52 & \\
\hline Reference & T91 & Balance & $8.00 \sim 9.50$ & 0.04 & $\begin{array}{c}0.30- \\
0.60\end{array}$ & $0.08 \sim 0.12$ & $0.20 \sim 0.50$ & 0.02 & 0.01 & $0.85 \sim 1.05$ & $0.06-.10$ \\
\hline Reference & HT9 & Balance & 12.1 & 0.51 & 0.57 & 0.2 & 0.17 & 0.016 & 0.003 & 1.04 & $<0.001$ \\
\hline Reference & $\begin{array}{c}\text { Inconel } \\
625 \\
\end{array}$ & $5.0 \max$ & $20.0-23.0$ & $\begin{array}{l}58.0 \\
\min \end{array}$ & 0.5 & 0.1 & 0.5 & 0.015 & 0.015 & $\begin{array}{l}8.0- \\
10.0 \\
\end{array}$ & $\begin{array}{c}3.15- \\
4.15 \\
\end{array}$ \\
\hline $\begin{array}{c}\text { Heat } \\
\text { X0A26AK21 }\end{array}$ & $\begin{array}{c}\text { Inconel } \\
625 \\
\end{array}$ & 0.52 & 22.08 & 64.32 & $<0.01$ & 0.01 & 0.04 & 0.002 & 0.001 & 9.03 & \\
\hline Heat D57540 & $\begin{array}{c}\text { Inconel } \\
693 \\
\end{array}$ & 3.92 & 28.04 & 63.03 & 0.29 & 0.009 & 0.1 & & 0.001 & & 0.68 \\
\hline Reference & $\begin{array}{c}\text { Inconel } \\
718\end{array}$ & 18.5 & 19 & 52.5 & - & - & & & & 3.05 & \\
\hline Heat 5 & $\begin{array}{c}\text { Inconel } \\
718 \\
\end{array}$ & 18.21 & 18.22 & 52.89 & 0.08 & 0.03 & 0.1 & 0.007 & 0.001 & 3.03 & \\
\hline Cast 34688 & Ni 99.2 & 0.05 & & 99.73 & 0.1 & & 0.03 & & 0.002 & & \\
\hline
\end{tabular}




\section{RESULTS AND DISCUSSION}

The results obtained during the reporting period are presented in two groups:

1. Study of oxide films on the candidate structural materials, including the use of an in situ technique based on the CDE approach, followed by postexamination of the composition, morphology, and thickness of the oxide films using SEM.

2. Fracture surface analysis and estimation of crack growth rates.

\section{STUDY OF OXIDE FILMS ON THE CANDIDATE STRUCTURAL MATERIALS}

The results of the study of the oxide films formed on austenitic stainless steels obtained during the reporting period follow:

1. Validation of the measured impedance spectra in symmetrical and asymmetrical CDE configurations.

2. Systematic data on the charge and mass transfer properties of the oxide films determined from CDE measurements.

3. Kinetic parameters of the charge and mass transfer processes in the oxide films and at the film/supercritical water interface determined from systematically measured data on film properties.

4. Ranking of candidate structural materials against their susceptibility to environmentally assisted degradation.

5. Chemical composition and thickness of the oxide films.

\section{Validation of the Measured Impedance Spectra}

Impedance measurements in the supercritical water have to overcome problems related to very low conductivity of the media between the CDE electrodes in the asymmetrical configuration. To obtain meaningful impedance spectrum, especially at low frequencies (suitable for generating data on partial charge and mass transfer processes), the amplitude of the injected AC signal must be sufficiently high.

The first step in validating the measured impedance spectra was to ensure that the AC amplitude's signal/noise ratio satisfied requirements of the linear perturbation theory and system causality requirements. The acceptable signal/noise ratio, which was defined on the basis of the 
repeatability of the impedance measurements, was about 5\% for high frequencies and $15 \%$ for low frequencies. The linearity condition was checked by measuring spectra at signal amplitudes ranging from 20 to $400 \mathrm{mV}$ (rms). The causality condition was checked using a K-K compatibility test based on Boukamp's procedure [61].

The signal/noise ratio in measured CDE spectra improves with increasing amplitude of the AC signal, especially at low $(<10 \mathrm{~Hz})$ frequencies (see Figure 43). The noise level is negligible for high-frequency $(>1 \mathrm{kHz})$ measurements with any amplitude in the $20-400 \mathrm{mV}$ range. A high level of noise precludes measurements with amplitude less than $100 \mathrm{mV}$ for frequencies less than $10 \mathrm{~Hz}$. The best signal/noise ratio was obtained when the amplitude was increased to $400 \mathrm{mV}$.

To select the AC signal amplitude in the 100-400 $\mathrm{mV}$ range, the average impedance was plotted for 4 measurements (amplitudes 100, 200, 300, and $400 \mathrm{mV}$ ) as shown in Figure 44. A standard deviation for each frequency was calculated and plotted as error bars (see Figure 44) and as relative variations of the impedance (see Figure 45). From these data, the AC amplitude $400 \mathrm{mV}$ was selected for CDE measurements in asymmetrical configuration. This amplitude provides an acceptable signal/noise ratio and an estimated nonlinearity not exceeding $6 \%$ for high frequencies and 12\% for low frequencies (see Figure 45 ).

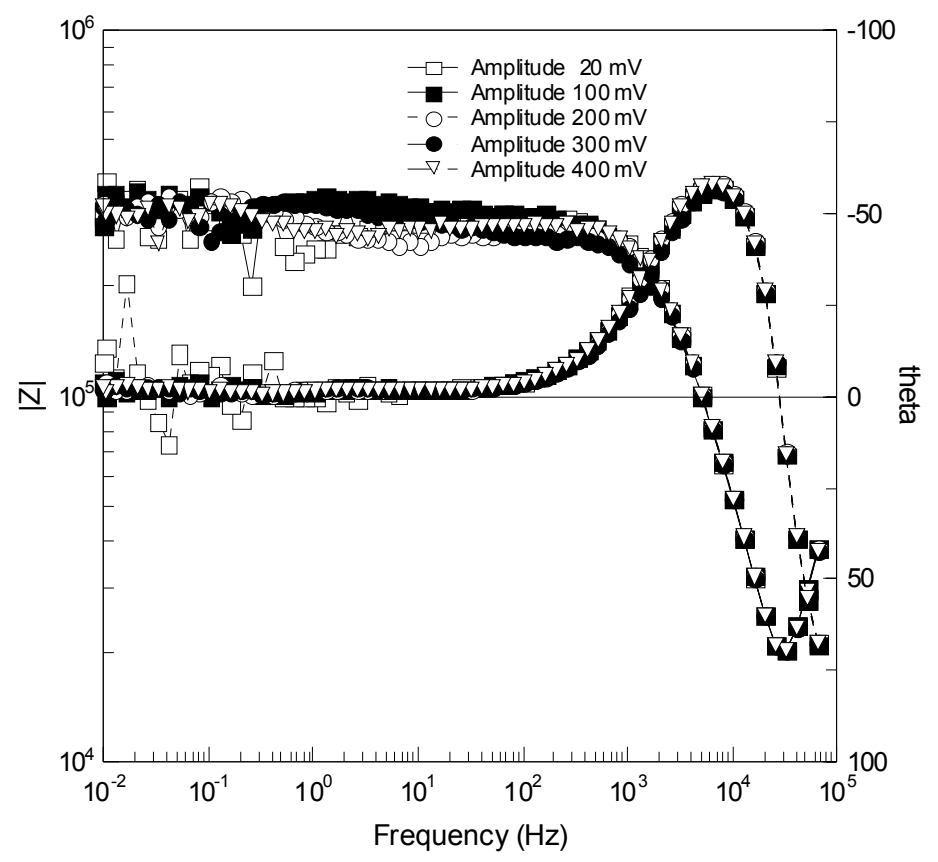

Figure 43. TLEC spectra measured for gradually increasing amplitude of the AC signal. 


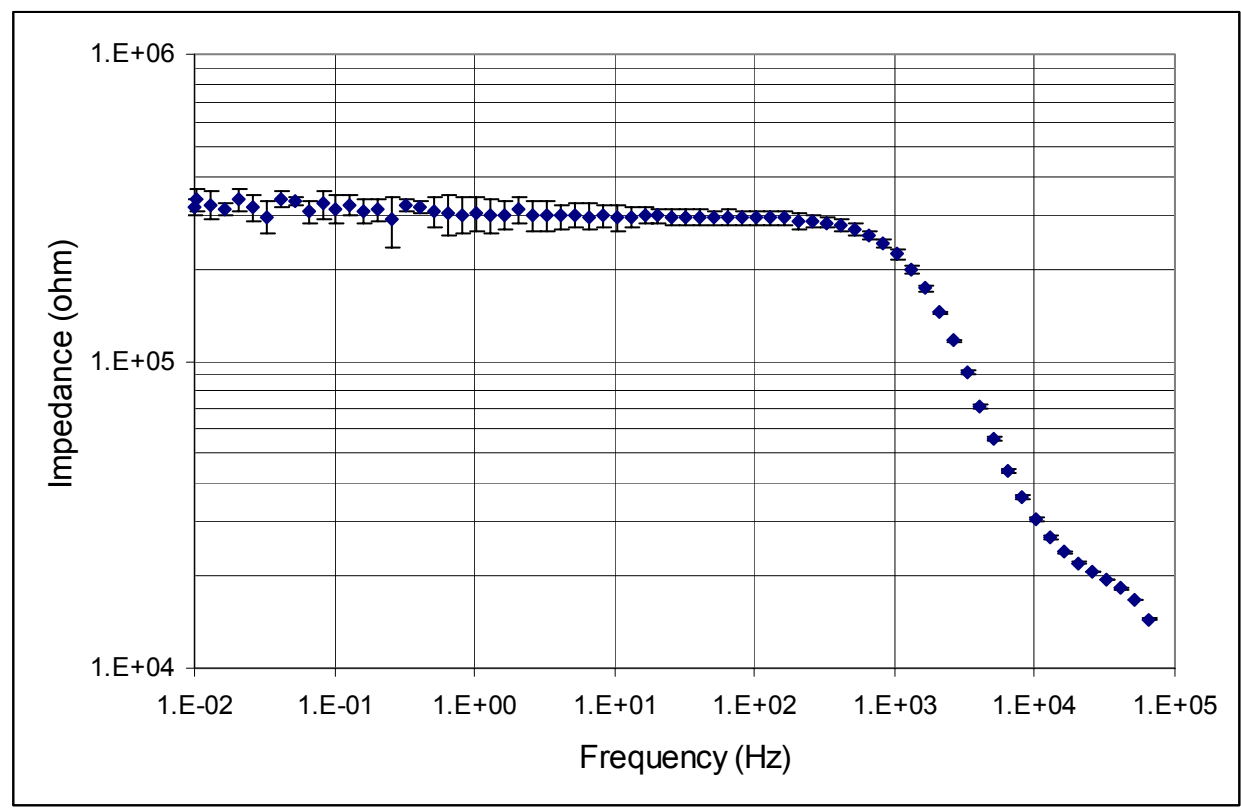

Figure 44. Average impedance and deviations for a set of measurements with AC signal amplitudes ranging from 100 to $400 \mathrm{mV}$.

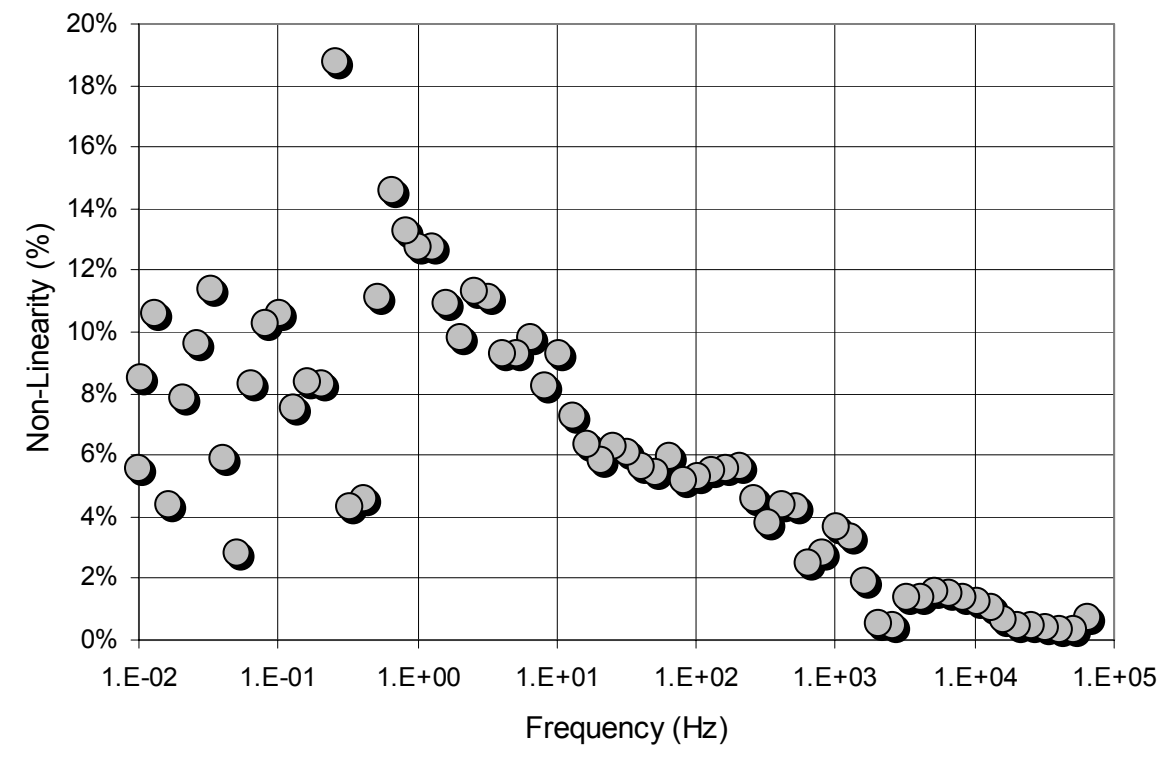

Figure 45. Percentage deviation in the impedance measured with an AC signal ranging from 100 to $400 \mathrm{mV}$. 
A similar procedure was repeated for CDE measurements in symmetrical configuration. Minimal sufficient amplitude ensuring acceptable signal/noise ratio was found to be $200 \mathrm{mV}$ (see Figure 46). As expected, this value was lower than the one for asymmetrical configuration (i.e., $400 \mathrm{mV}$ ), given that the metal/film/metal system is more conductive than the metal/film/electrolyte.

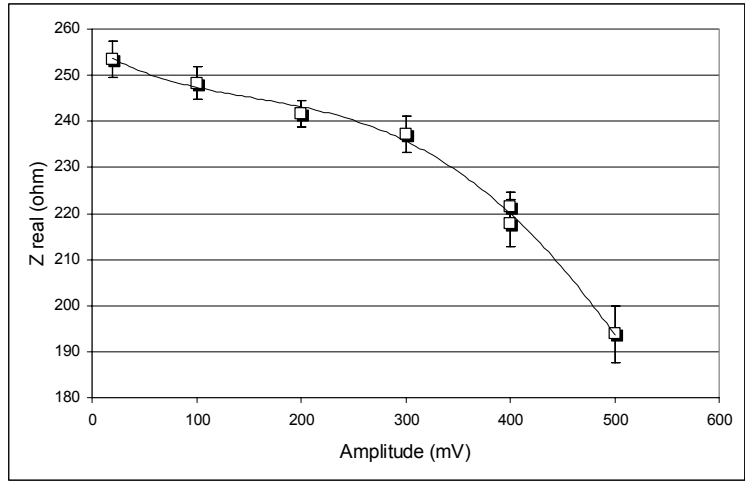

(a)

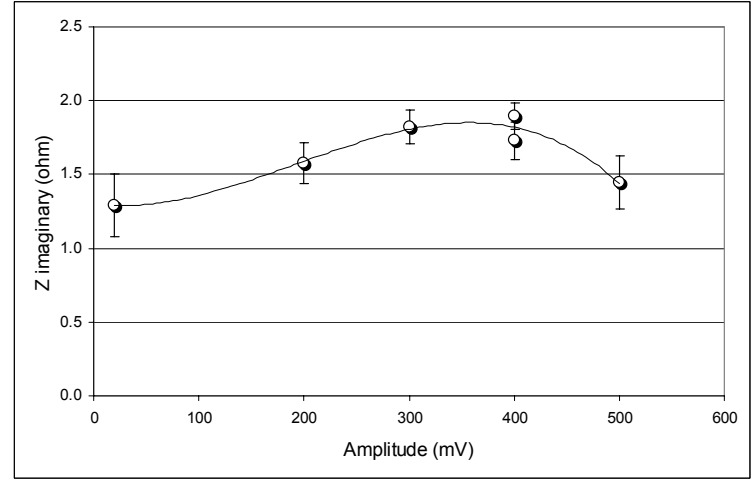

(b)

Figure 46. Variations in the real and imaginary part of the CDE impedance measurements for a single frequency $(1 \mathrm{~Hz})$ with amplitude of the AC signal.

CDE spectra measured with minimal sufficient AC amplitudes in symmetrical and asymmetrical configurations were tested for causality by applying the K-K transform to the real and imaginary components (see Figure 47). Good agreement between the measured and K-K calculated real and imaginary part indicated that no apparent instability existed in the examined electrochemical systems and that the output from the system was exclusively the result of perturbing the AC input signal.

The possible artifacts arising from the nature of the solid-solid contacts have been considered in some detail and validation criteria have been proposed [41, 62]. Briefly, to consider an impedance spectrum measured in the contact situation to be valid, its low-frequency limit should be independent of the contact pressure applied to the electrodes, and it should be equal to the value measured by a four-point DC method to exclude the influence of the spreading resistance of the contact on the frequency response.

Both criteria were checked by comparing the measured impedance spectra at gradually increasing contact pressure with the resistance measured by the CER technique, which is a fourpoint measurement method $[32,33]$. Both the low-frequency limit of the impedance and the CER-measured DC resistance have been found first to decrease rapidly with increasing contact 
pressure and than asymptotically to approach a value, which depends on the state of the oxide film.

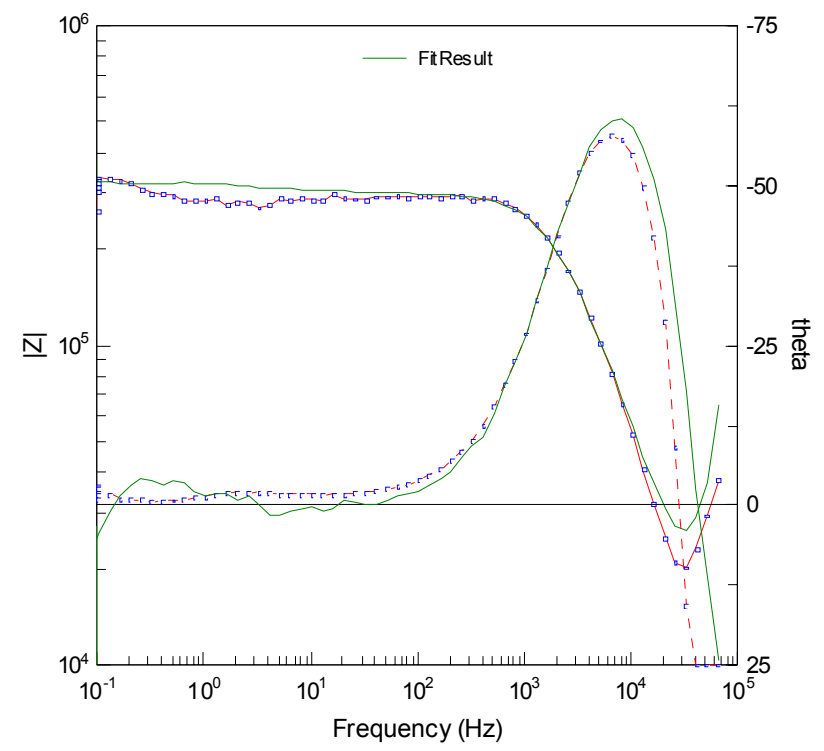

(a)

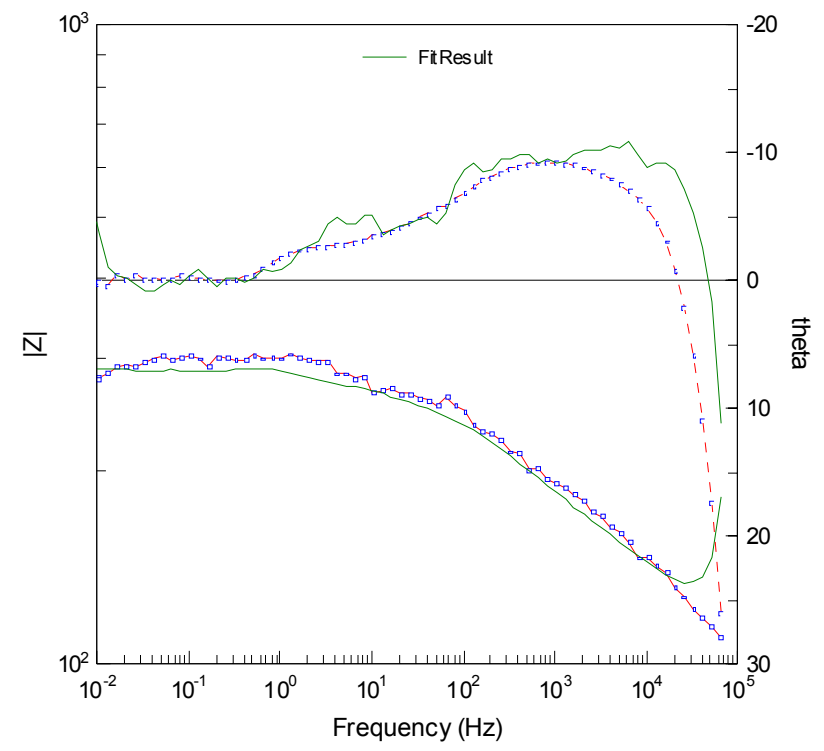

(b)

Figure 47. K-K transform causality test results for impedance spectra measured for asymmetrical (a) and symmetrical (b) configurations. 
A minimal contact pressure corresponding to the diminishing first derivative $\mathrm{dR} / \mathrm{dx}$ was used for contact CDE measurements. Good agreement between CER resistance and lowfrequency contact impedance was observed in all cases. For example, the low-frequency limit for the spectrum shown in Figure $47 \mathrm{~b}$ is $\sim 250 \mathrm{ohm}$, which is in good agreement with the 220 ohm observed in CER measurements performed immediately after the contact spectra were measured.

\section{Collection of data on the charge and mass transfer properties of the oxide films}

Information on the oxide films was acquired by measuring the low-frequency part of the impedance spectra in asymmetrical configuration (TLEC) and the entire frequency range of the impedance spectra in symmetrical configuration (CEI). ${ }^{i}$ The highest frequency in all measurements was $65,000 \mathrm{~Hz}$. The lowest frequency was between 1 and $10 \mathrm{mHz}$. Electronic charge carriers were blocked in asymmetrical and unblocked in symmetrical configuration.

Figure 48a shows a typical TLEC spectrum. The large peak in the phase angle, spanning the frequency range from hundreds of $\mathrm{Hz}$ to tens of $\mathrm{kHz}$, is associated with an $\mathrm{R}(\mathrm{CR})$ type of impedance of the gap filled with supercritical water between the samples. In the range above 30 $\mathrm{kHz}$, measured impedance corresponded to the inductive behavior of the connecting wires. A low-frequency part of the spectra, which carried the information on the oxide film, is shown in Figure 48b. Three impedance spectra (1 to 3), collected sequentially, are plotted in Figure 48 to demonstrate the good repeatability of the TLEC measurements for the entire frequency range.

The low-frequency part of each TLEC spectra was first examined for the presence of "time constants" (or peaks and peaks asymmetry in the frequency dependence of the phase angle). The formation and growth of the time constants in the measured TLEC spectra provide in situ information on the growth of the oxide films (see Figure 49a). The lower curve "NO FILM" in Figure 49a corresponds to the measurement performed for a fresh sample. The three other curves correspond to the measurements performed at various time points $\left(t_{1}>t_{2}>t_{3}\right)$ during the exposure of samples to supercritical water. This is a very powerful feature of the TLEC technique, especially taking into account the noninvasiveness ${ }^{\mathrm{ii}}$ of the TLEC measurements.

Contact impedance spectra measured in symmetrical configuration (see Figure 49b) were more sensitive to the presence of the oxide film. A typical spectrum obtained for a fresh sample ("NO FILM" in Figure 49b) exhibited the system's pure inductive (high frequencies) and ohmic (low frequencies) behavior, with the modulus of impedance not exceeding a few ohms, which

\footnotetext{
${ }^{\mathrm{i}}$ Several hundred impedance spectra were measured during the reporting period in asymmetrical and symmetrical configurations to study the oxide films formed on austenitic steels, nickel alloys, and ferritic-martensitic steels.

ii TLEC measurements were performed without contacting the oxide film.
} 
clearly indicated the absence of a measurable oxide layer on the surface of the CDE samples. Once the film was formed, the modulus of impedance increased by a factor of $10^{3}$ into the kohm range, and several time constants were observed: one at $\sim 10 \mathrm{~Hz}$ and another at $\sim 100 \mathrm{~Hz}$ (see Figure 49b).

Monitoring of the formation and growth of the oxide films was found to be less invasive if periodic TLEC measurements were performed. The typical sampling interval for such measurements was between $0.5 \mathrm{~h}$ and 4 days. The majority of the TLEC measurements were performed in automatic "batch mode," which allowed data accumulation $24 \mathrm{~h} / \mathrm{d}$. CEI measurements were performed between TLEC measurements. The first measurement provided a "baseline" for a fresh sample ("NO FILM"). The following measurements provided information on the growing oxide film.

First, information on the transport properties of the oxide films was deduced from the magnitude of the impedance moduli and location of time constants in the frequency dependence of the phase angle. (Figure 47 shows a typical pair of the TLEC and CEI spectra.). The impedance spectra in both configurations comprised two time constants. The high-frequency time constant is ascribed to the electronic properties of the space-charge layer in the oxide. The low-frequency time constant is associated with the solid-state transport of point defects.

A time constant for impedance measured in symmetrical configuration, $\tau_{W, S}$ (see Figure $47 \mathrm{~b}$ and Figure 49b) was observed for frequencies 1 or 2 orders of magnitude higher than $\tau_{W, A}$ (see Figure 47a and Figure 48b and Figure 49a, which zoom in on the low-frequency region). Accordingly, the transport process associated with time constant $\tau_{W, S}$ is related to current carriers that attain their steady state transport rate faster than those associated with $\tau_{W, A}$ in the asymmetrical configuration. This observation indicates that, for measurements at supercritical temperatures in the asymmetrical configuration, the ionic point defects were the unblocked current carriers, whereas in the symmetrical configuration this role was played by the electronic defects.

The values of the impedance at low frequencies are 1 to 2 orders of magnitude lower in the symmetrical configuration (see Figure 47b) than in the asymmetrical one (see Figure 47a), indicating that the steady state conductivity of the system is higher in the symmetrical configuration. This finding also confirms our expectation of a predominant role for the electronic carriers in the symmetrical configuration and the ionic carriers in the asymmetrical configuration at supercritical temperatures.

In summary, the picture of the oxide that emerges from the impedance measurements is that of a mixed-conducting film with predominant electronic conductivity. 


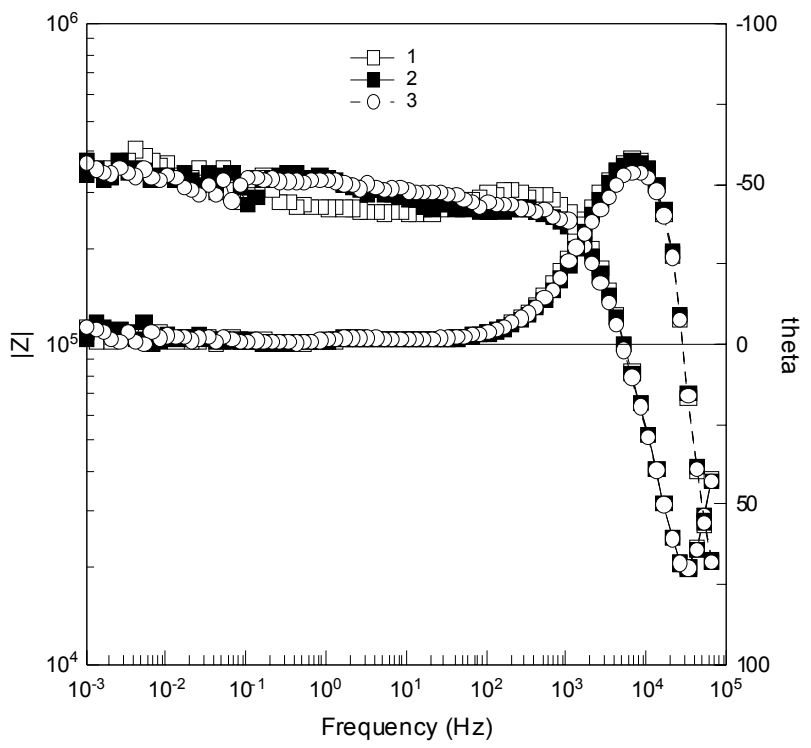

(a)

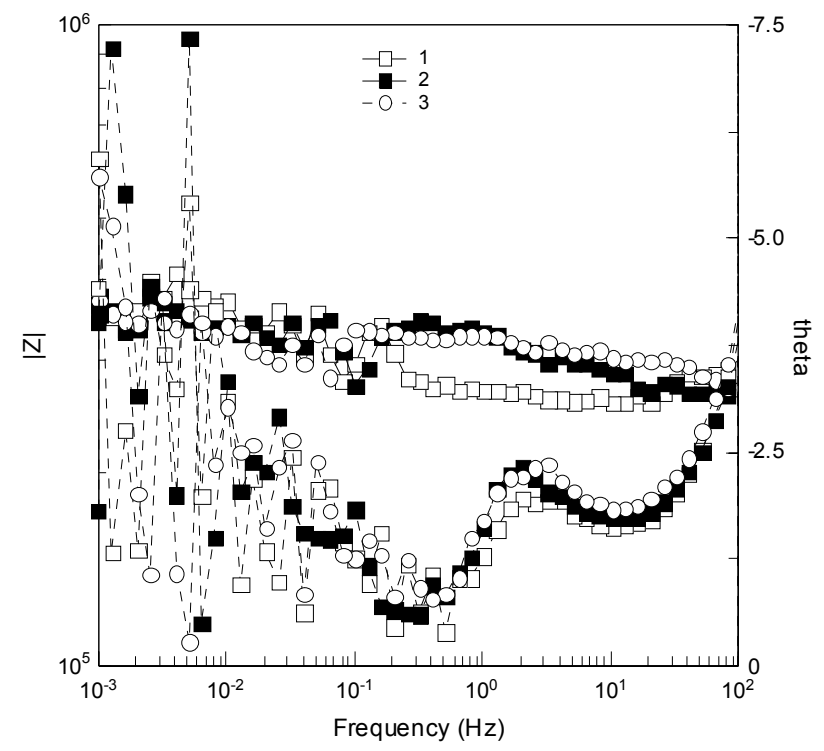

(b)

Figure 48. TLEC spectra measured in symmetrical configuration: the entire frequency range (a) and the low-frequency portion of the range (b). 


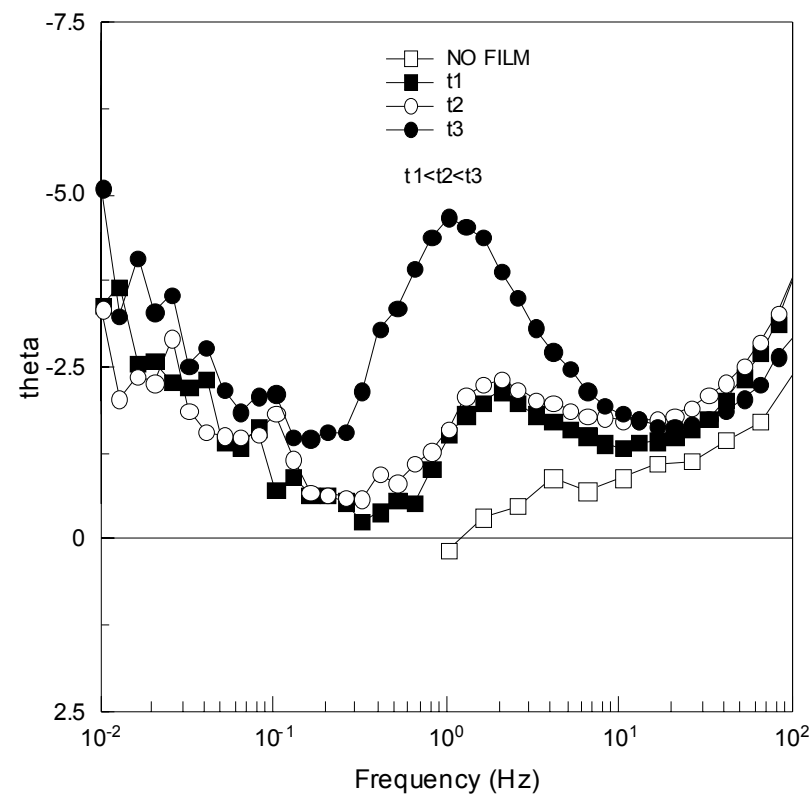

(a)

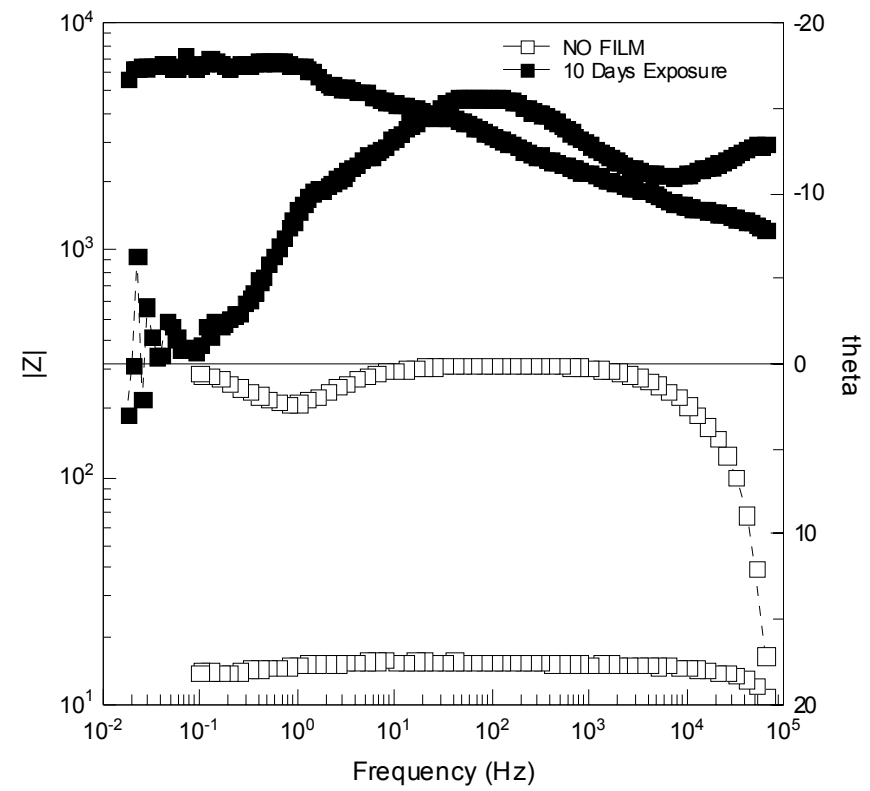

(b)

Figure 49. Evolution of impedance spectra measured in the asymmetrical (a) and the symmetrical (b) configuration with formation and growth of the oxide film. 
The impedance spectra in the asymmetrical and symmetrical configuration were fitted to the transfer functions described by Eq. (1)-(6). Figure 50 shows the equivalent circuits representing oxide film and film/supercritical water interface. A complete system (connecting wires, supercritical water between the samples, and oxide film) can also be represented as the equivalent circuits shown in Figure 51. The kinetic and thermodynamic properties of the oxide films and film/supercritical water interface determine the parameters and their values of circuit elements. Electrochemical mechanisms and topologies are mainly reflected in the connection schemes.

Two connection schemes-Model 1 and Model 2- were explored. The difference between them was physical: for Model 1, we considered the bulk film with a space charge layer capacitance in parallel to the migration resistance and Warburg impedance due to transport described by Nernst-Planck equations. For Model 2, we considered the interface as a parallel combination of a capacitance and a charge transfer resistance, whereas in the bulk film we envisioned only Warburg type diffusion (i.e., the oxide is an electronic conductor and no space charge is present).

Diffusion processes were represented as a finite Warburg element. This type of circuit element describes one-dimensional diffusion, and it does not have an electric analog. Charge transfer processes were represented by an electric resistor. The capacitance was represented in three ways: an electric capacitor, a constant phase element (or nonideal capacitor), and Young impedance. Young impedance describes an insulating or semiconducting layer in which a steady-state profile of defects stretches from an interface to the bulk and gives rise to an exponential conductivity profile [35].

The measured impedance spectra in symmetrical and asymmetrical configurations were fitted to the oxide film models represented by Model 1 and Model 2 using Zview fitting capabilities. A particular fit was accepted if $\chi^{2}<10^{-2}$ and uncertainties in the obtained parameters of circuit elements did not exceed $10-15 \%$.

An example of the best-fit results is shown with solid lines in Figure 52 and Figure 53, demonstrating the ability of transfer functions [Eq. (1)-(6)] to account for the data. 

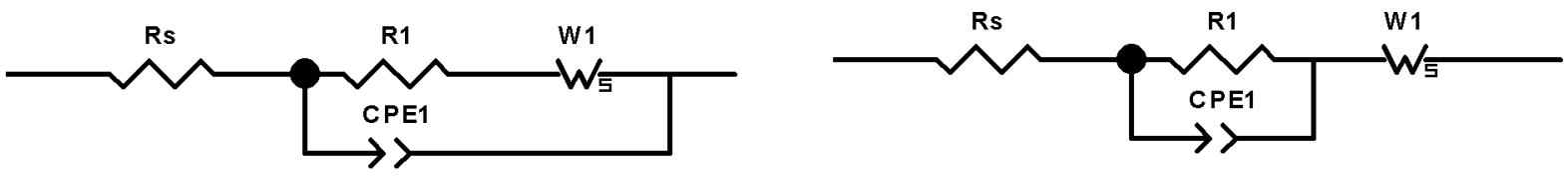

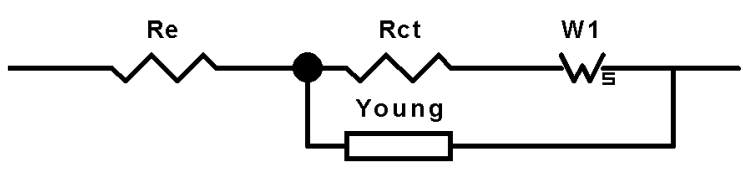

(a)

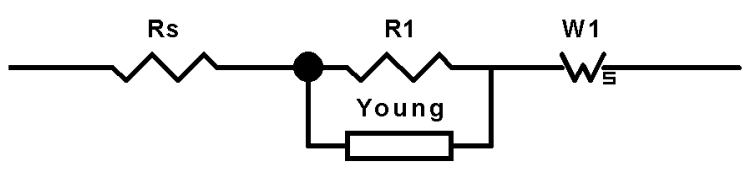

(b)

Figure 50. Equivalent circuits of the transfer functions of the oxide films: Model 1 (a) and Model 2 (b).

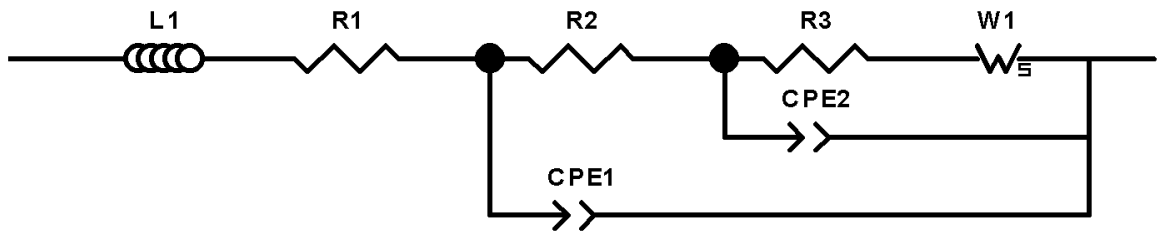

(a)

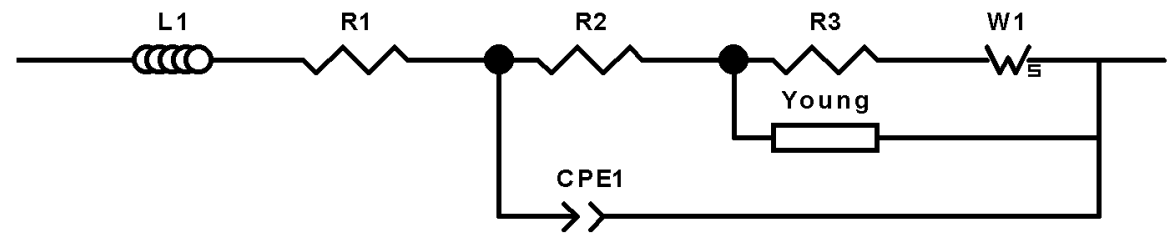

(b)

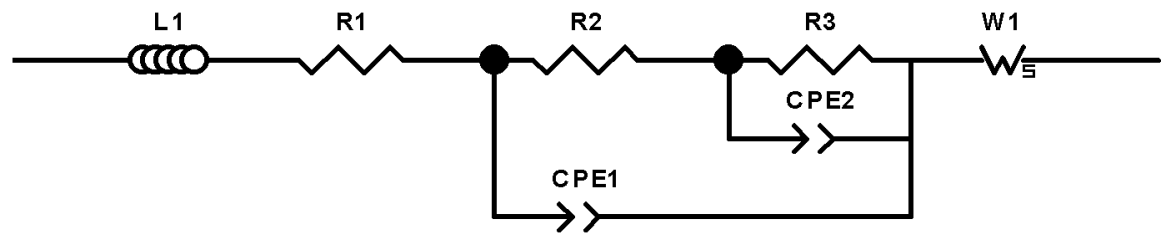

(c)

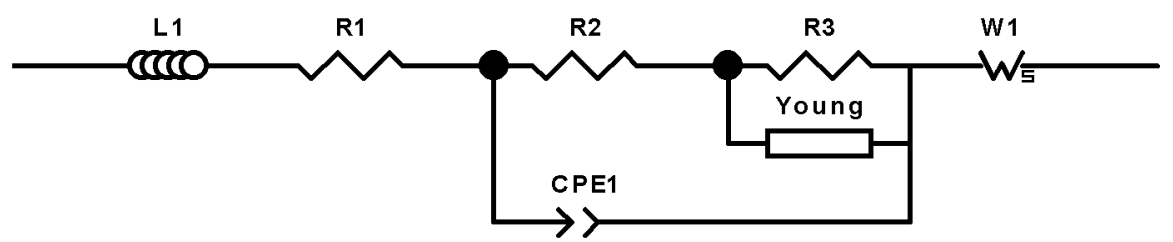

(d)

Figure 51. Equivalent circuits for the complete system: Model 1 (a, b) and Model 2 (c, d). 


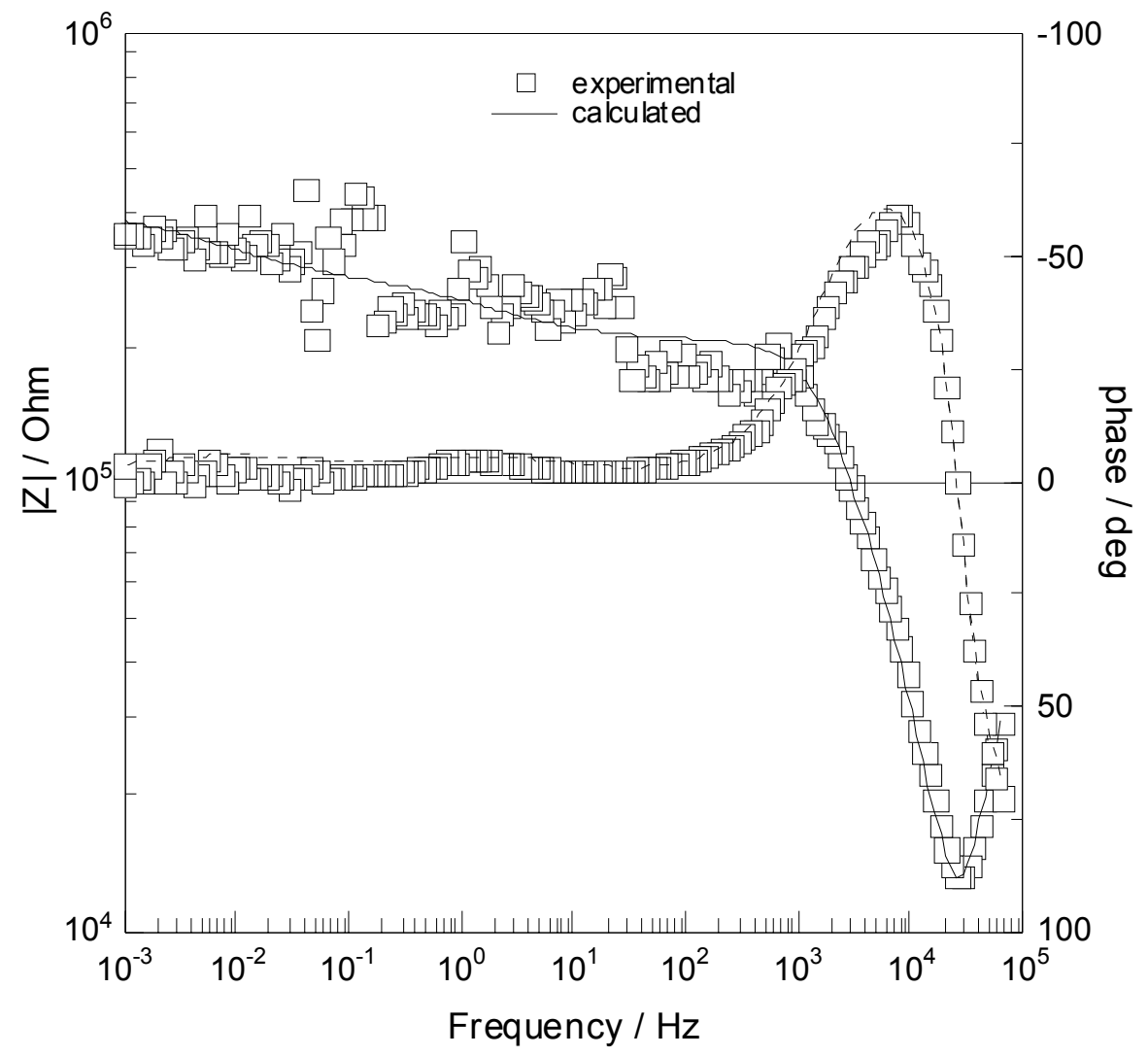

(a)

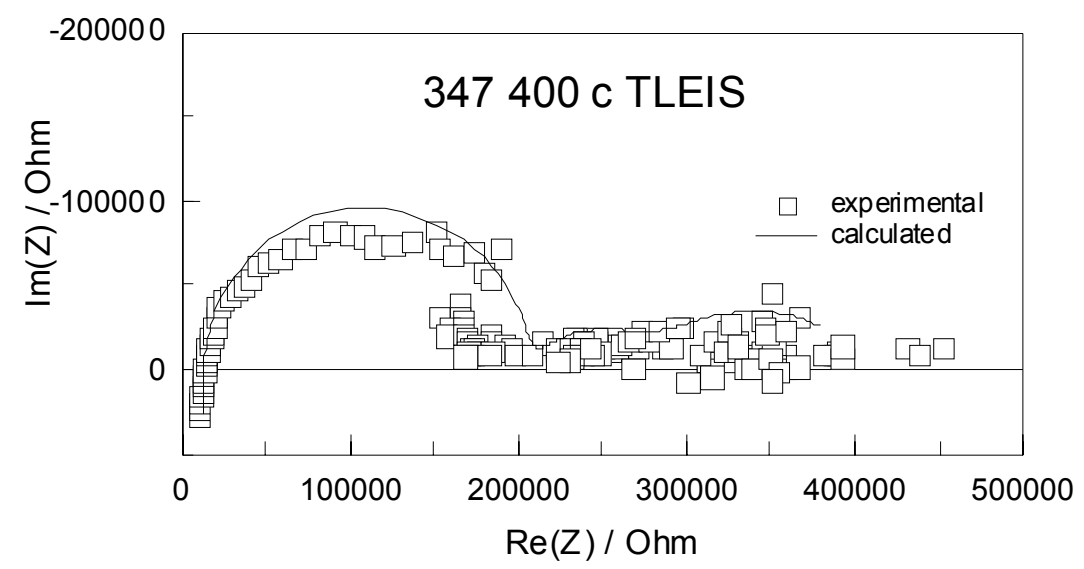

(b)

Figure 52. Comparison of measured and calculated impedance spectra for the asymmetrical configuration. 


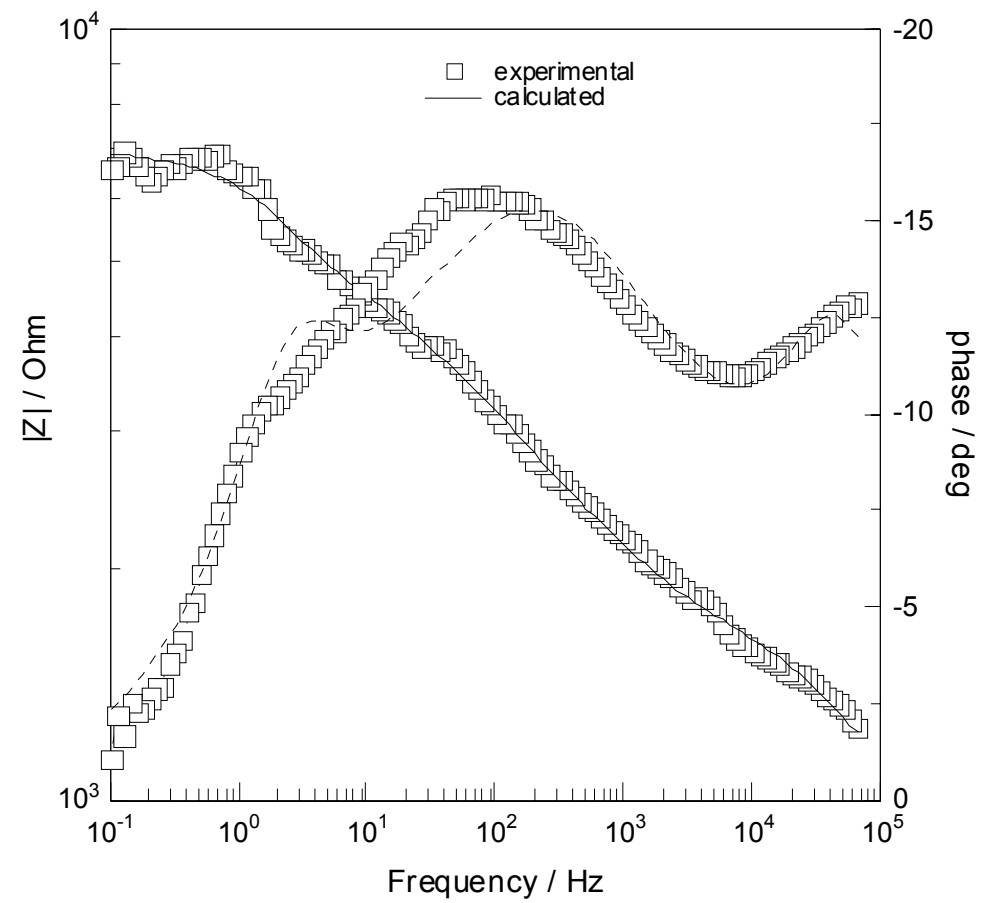

(a)

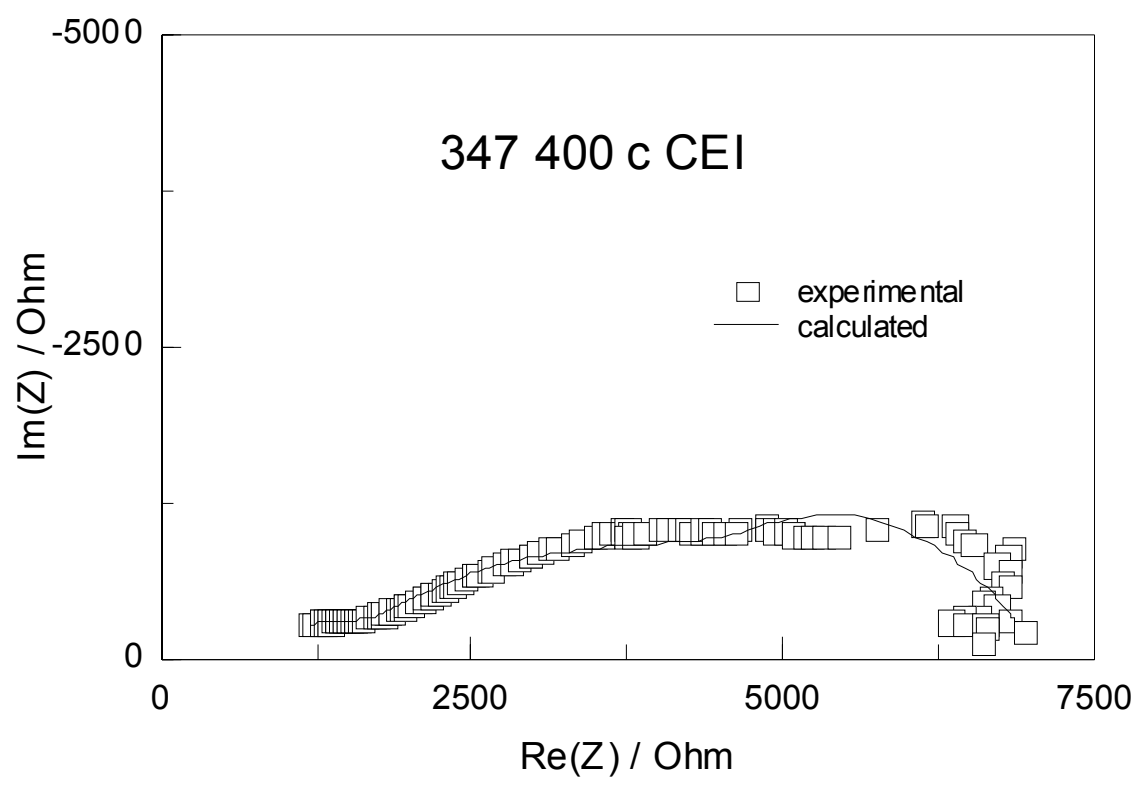

(b)

Figure 53. Comparison of measured and calculated impedance spectra for the symmetrical configuration. 


\section{Validation of kinetic parameters of the charge and mass transfer processes in the oxide films}

The kinetic parameters derived from TLEC impedance measurements were first compared with independent measurements performed at VTT. Because VTT performed CDE measurements only at subcritical temperatures, a special set of TLEC measurements at $150^{\circ} \mathrm{C}$ and $300^{\circ} \mathrm{C}$ (see Figure 54) were performed for comparison with the VTT results. Table 4 presents kinetic parameters calculated at SRI and compares them with kinetic parameters obtained at VTT under similar conditions.

At VTT, the value for the diffusion coefficient of pure Fe in $0.1 \mathrm{M} \mathrm{Na}_{2} \mathrm{~B}_{4} \mathrm{O}_{7}$ at $200^{\circ} \mathrm{C}$ was found to be on the order $10^{-13} \mathrm{~cm}^{2} \mathrm{~s}^{-1}$. On the other hand, the Warburg coefficient in the same solution for AISI 316 has been evaluated to be about $200-400 \Omega \mathrm{cm}^{2} \mathrm{~s}^{-1}$ at $150^{\circ} \mathrm{C}$ and $400-500$ $\Omega \mathrm{cm}^{2} \mathrm{~s}^{-1}$ at $300^{\circ} \mathrm{C}$. Given that the SRI measurements were conducted in pure water, the correspondence between the results is rather good.

The area in the calculations was $0.08 \mathrm{~cm}^{2}$ (50\% of the nominal), and the electric field strength was $10^{5} \mathrm{~V} / \mathrm{cm}$. The electric field strength had to be assumed as long as we did not have precise information about the oxide film thickness. Therefore, it could have been lower or higher, which would then change the value of the diffusion coefficient accordingly. 


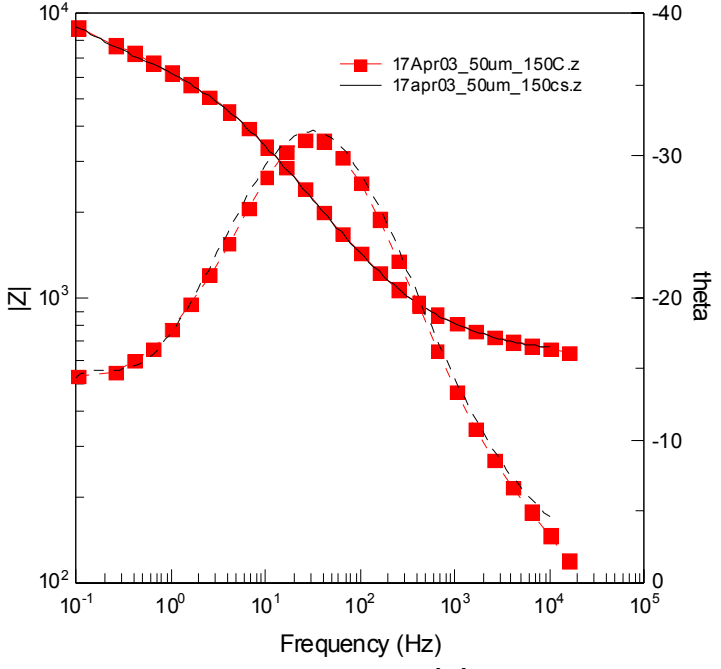

(a)

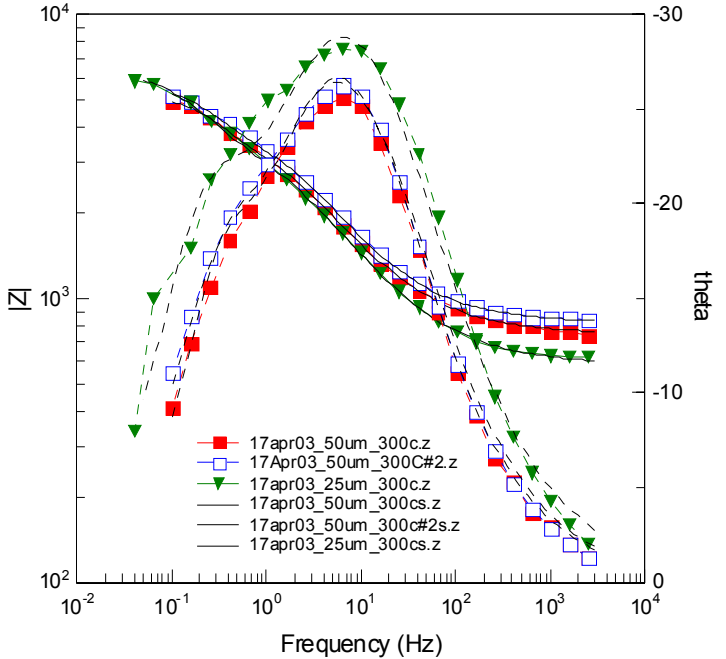

(b)

Figure 54. TLEC impedance spectra for AISI316 at $150^{\circ} \mathrm{C}$ and distances of $50 \mu \mathrm{m}$ (a) and at $300^{\circ} \mathrm{C}$ and distances of 25 and $50 \mu \mathrm{m}$.

Table 4. Average Warburg impedance parameters obtained from simulations and calculations.

\begin{tabular}{|c|c|c|c|c|}
\hline \multicolumn{2}{|c|}{ Parameter } & $\begin{array}{c}\text { Temperature }\left({ }^{\circ} \mathrm{C}\right) / \\
\text { distance }(\mu \mathrm{m})\end{array}$ & $\begin{array}{c}\text { Temperature }\left({ }^{\circ} \mathrm{C}\right) / \\
\text { distance }(\mu \mathrm{m})\end{array}$ & $\begin{array}{c}\text { Temperature }\left({ }^{\circ} \mathrm{C}\right) / \\
\text { distance }(\mu \mathrm{m})\end{array}$ \\
\hline & & $150 / 50$ & $300 / 25$ & $300 / 50$ \\
\hline \multicolumn{2}{|l|}{ W-R $(\Omega)$} & 5833 & 3500 & 1930 \\
\hline \multicolumn{2}{|l|}{ W-T (s) } & 6.4 & 2.8 & 1.65 \\
\hline \multicolumn{2}{|l|}{ W-P } & 0.43 & 0.46 & 0.5 \\
\hline \multirow{3}{*}{$\begin{array}{l}\text { SRI results } \\
\text { for pure } \\
\text { water }\end{array}$} & & & & \\
\hline & $\begin{array}{l}\text { Diffusion } \\
\text { coefficient D, } \\
\left(\mathrm{cm}^{2} \mathrm{~s}^{-1}\right)\end{array}$ & $2.1 \times 10^{-14}$ & $8.7 \times 10^{-14}$ & $1.5 \times 10^{-13}$ \\
\hline & $\begin{array}{l}\text { Warburg } \\
\text { coefficient } \sigma \text {, } \\
\left(\Omega \mathrm{cm}^{2} \mathrm{~s}^{-1}\right)\end{array}$ & 210 & 174 & 120 \\
\hline \multirow{2}{*}{$\begin{array}{l}\text { VTT results } \\
\text { for sodium } \\
\text { tetraborate } \\
\text { solution }\end{array}$} & $\begin{array}{l}\text { Diffusion } \\
\text { coefficient D, } \\
\left(\mathrm{cm}^{2} \mathrm{~s}^{-1}\right)\end{array}$ & \multicolumn{3}{|c|}{$\sim 10^{-13}$ at $200^{\circ} \mathrm{C}$} \\
\hline & $\begin{array}{l}\text { Warburg } \\
\text { coefficient } \sigma, \\
\left(\Omega \mathrm{cm}^{2} \mathrm{~s}^{-1}\right)\end{array}$ & $200-400$ & \multicolumn{2}{|c|}{$400-500$} \\
\hline
\end{tabular}




\section{Oxide film transport properties for stainless steel 347}

Film formation and growth was monitored for steady state and time dependent oxidizing conditions to obtain in-situ real time information on materials susceptibility to SCC in terms of transport resistance of the oxide films and repassivation time. The process of growth of the oxide film on 347 stainless steel at various oxidizing conditions (dissolved oxygen concentrations) in illustrated in Figure 55 and Figure 56.

Data on the electronic transport properties of the oxide film (Figure 55) indicate that a "critical" level of dissolved oxygen may exist for each structural material. In case of 347 stainless steel, increase in dissolved oxygen concentration from $0.1 \mathrm{ppm}$ to $0.4 \mathrm{ppm}$ resulted in formation of significantly less protective (or more electronically conductive) oxide film. Further increase in dissolved oxygen concentration from $0.4 \mathrm{ppm}$ to $1.5 \mathrm{ppm}$ does increase electronic conductivity of the oxide, but to a much lesser extent. Impact of critical oxygen levels on selection of structural materials should be quantified further using a combination of radiolysis and SCC modeling. A practical outcome of such an effort would be an optimal selection of the structural materials in the heat transport circuit under as low as reasonable achievable local concentrations of the radiolytically produced oxidizing species.

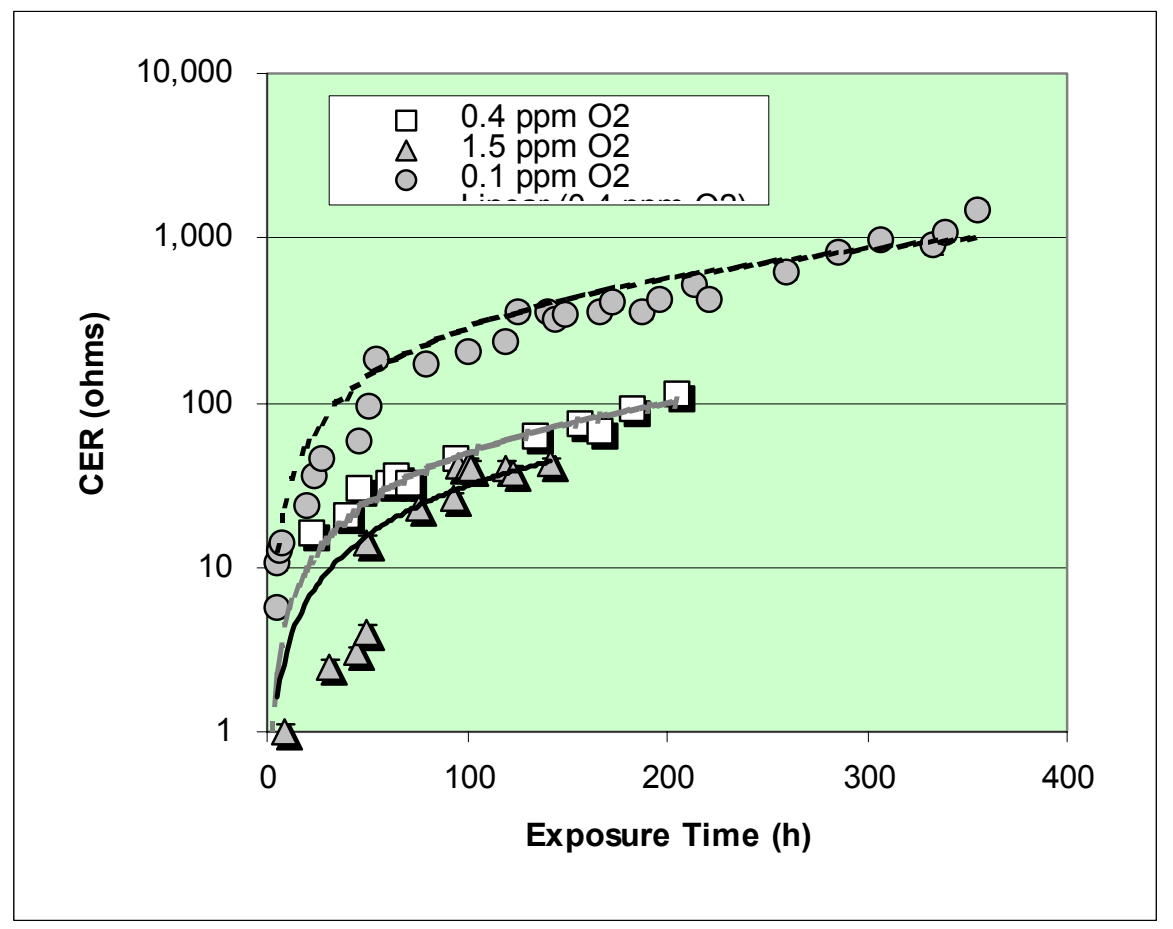

Figure 55. Illustration of "critical level" of oxidizing conditions in supercritical water on electronic properties of the oxide film formed on 347 stainless steel. 


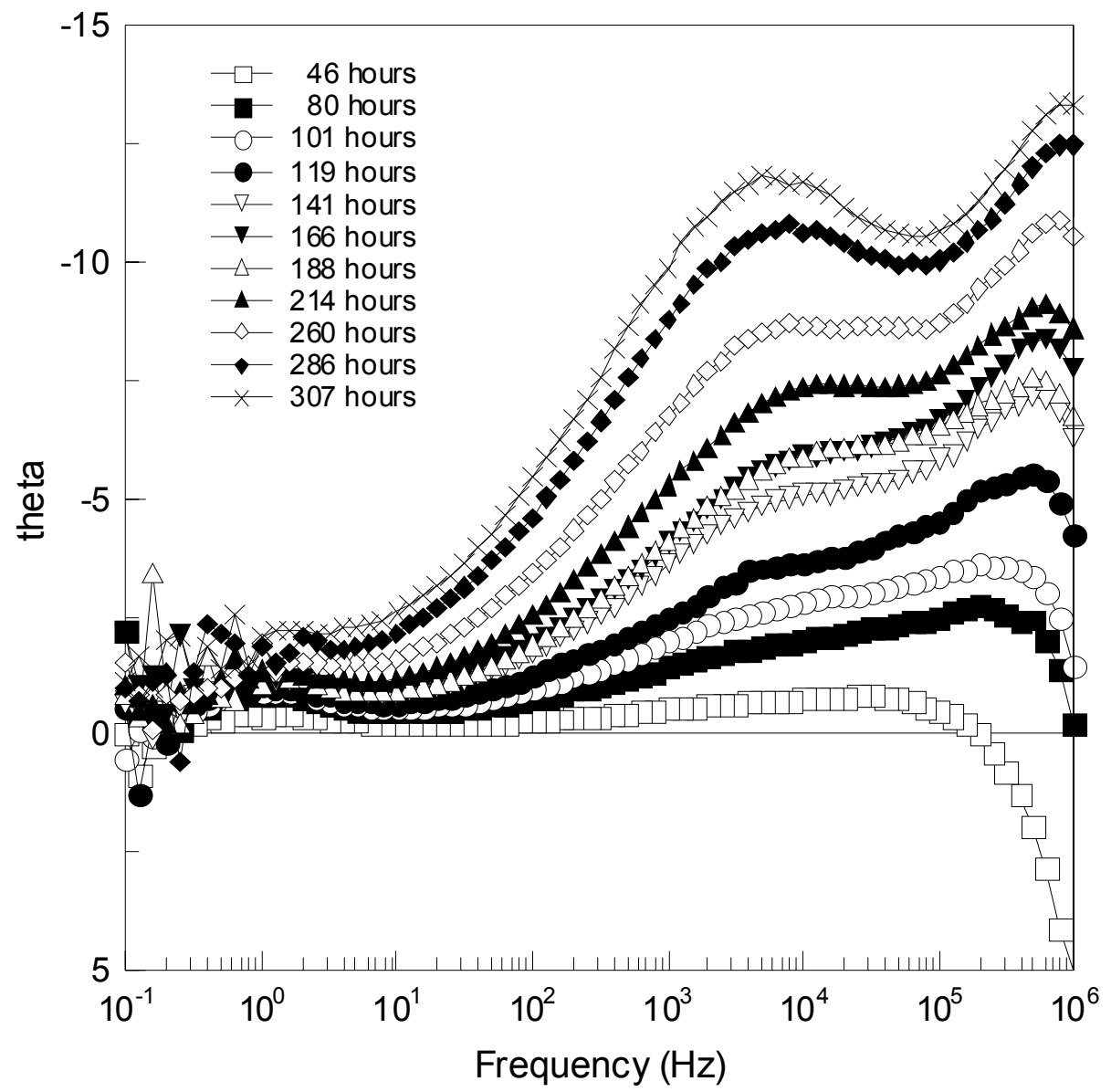

Figure 56. Evolution of the phase angle during CEI measurement of the oxide film growth on 347 stainless steel in supercritical water. Concentration of dissolved oxygen is $100 \mathrm{ppb}$

In-situ data on evolution of the pahse angle of the measured CEI spectra with thime contains (Figure 56) information on roles of the charge and mass transport processes. The characteristic frequency of the faster process is between $10^{4}$ and $10^{6} \mathrm{~Hz}$. Such a high frequency strongly suggests an electronic process, which may be either an electron transfer through the interfaces or the relaxation of the electronic space charge layer. The lower characteristic frequency at around $10-10^{3} \mathrm{~Hz}$ is most probably related to a transport process. It is reasonable to connect this process with the diffusion-migration transport of defects within the film. Formation of a pronounced high frequency time constant may be observed after 80 hours of exposure. A low frequency time constant becomes discernible at a later time - after 140-160 hours of exposure. 
Evolution of the impedance parameters of the oxide film formed on 347 stainless steel exposed to supercritical water under varous oxidizing conditions is shown in Figure 57. Stability of the oxide film is decreasing with increasing in concentration of dissolved oxygen, as may be judged by the lower values of all impedance parameters in Figure 57b and Figure 57c in comparision with Figure 57a. Charge transfer plays a dominant role at low oxidizing conditions. At higher dissolved oxygen levels roles of charge transfer and diffusion become comparable. Electronic conductivity of the film obtained as $|\mathrm{Z}|_{\mathrm{f} \rightarrow 0}$ is increasing, which is in agreement with CER data in Figure 55. Agreement with CER data is often used as a confirmation of the validity of measured CEI spectra.

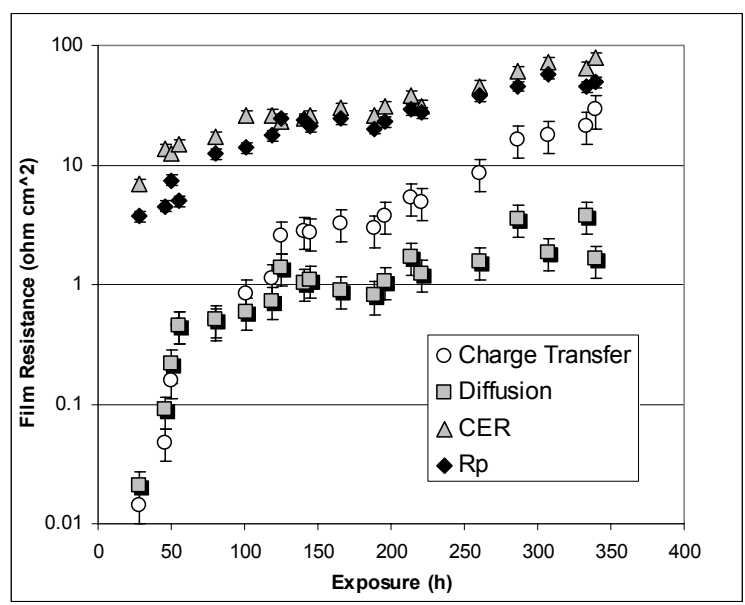

(a)

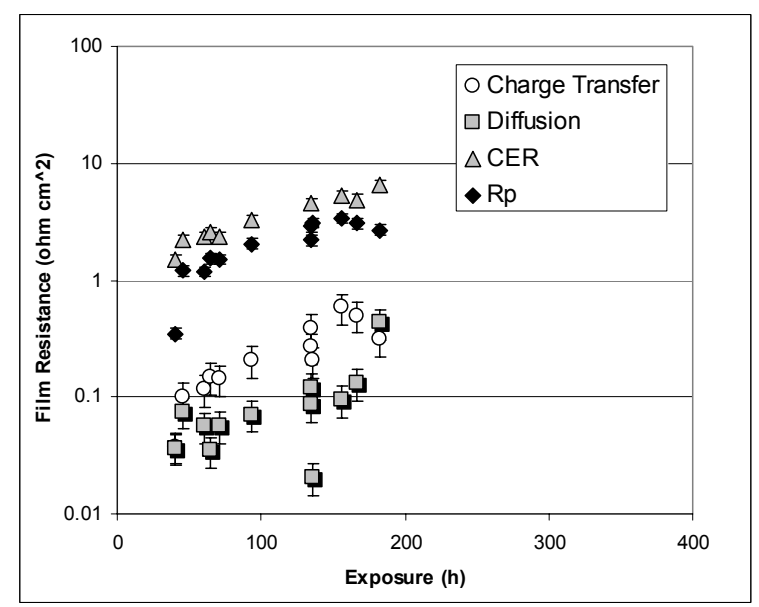

(b)

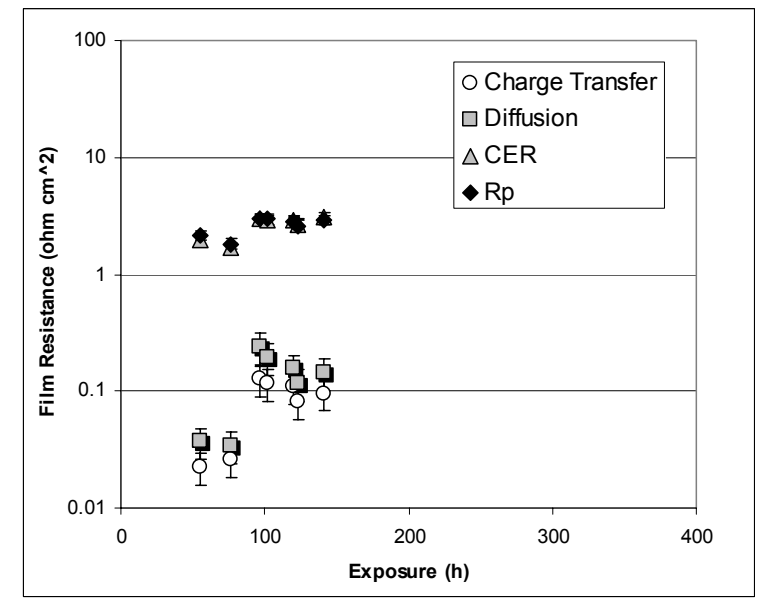

(c)

Figure 57. Impedance parameters of the oxide film formed on 347 stainless steel exposed to supercritical water: charge transfer resistance, diffusion (Warburg) resistance, contact electric resistance (CER) and polarization resistance $\left(R_{p}\right)$. Concentrations of dissolved oxygen: (a) - $100 \mathrm{ppb}$, (b) - $400 \mathrm{ppb}$, and (c) $1400 \mathrm{ppb}$. 
Capacitive properties of the oxide films formed at various oxidizing condition are illustrated by Figure 58, which depicts change in Young's impedance of the film with time. Obtained in-situ data on Young's impedance allowed to reconstruct growth of the oxide films in terms of thickness of the layer in which point defect transport occurs (Figure 59). Impact of oxidizing conditions manifests itself as decrease in the layer's thickness with increase in dissolved oxygen concentration. At the same time, the layer forms slightly faster for higher dissolved oxygen concentrations. Data in Figure 55 and Figure 59 were used to estimate repassivation time required for crack growth analysis as discusses later in the text.

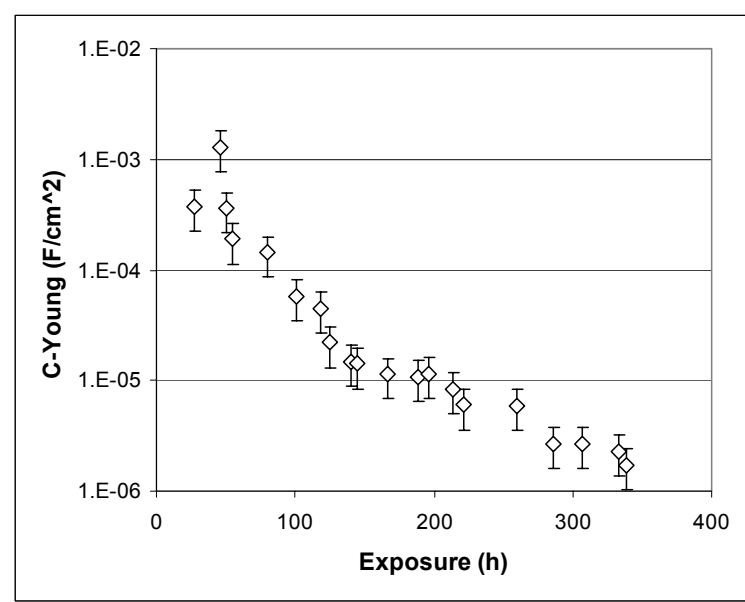

(a)

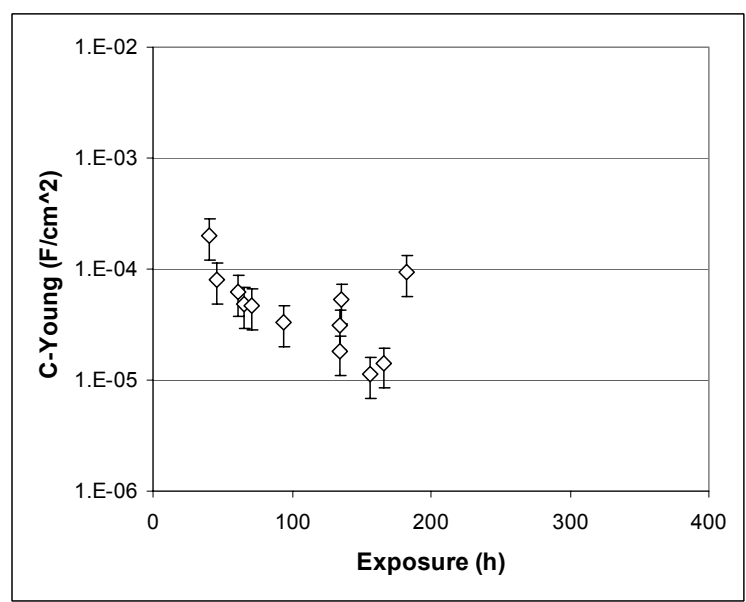

(b)

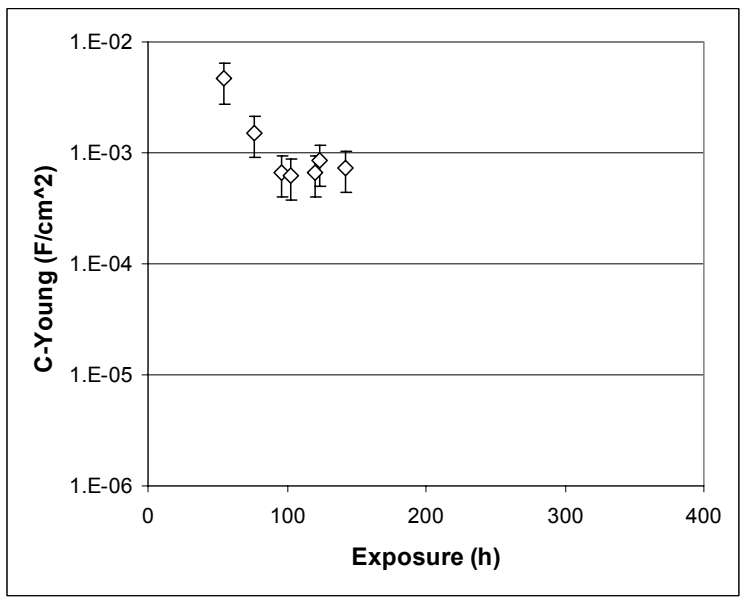

(c)

Figure 58. Effect of supercritical water oxidizing conditions on Young's impedance of the oxide film growing on 347 stainless steel. Concentrations of dissolved oxygen: (a) - $100 \mathrm{ppb}$, (b) - $400 \mathrm{ppb}$, and (c) $-1400 \mathrm{ppb}$. 


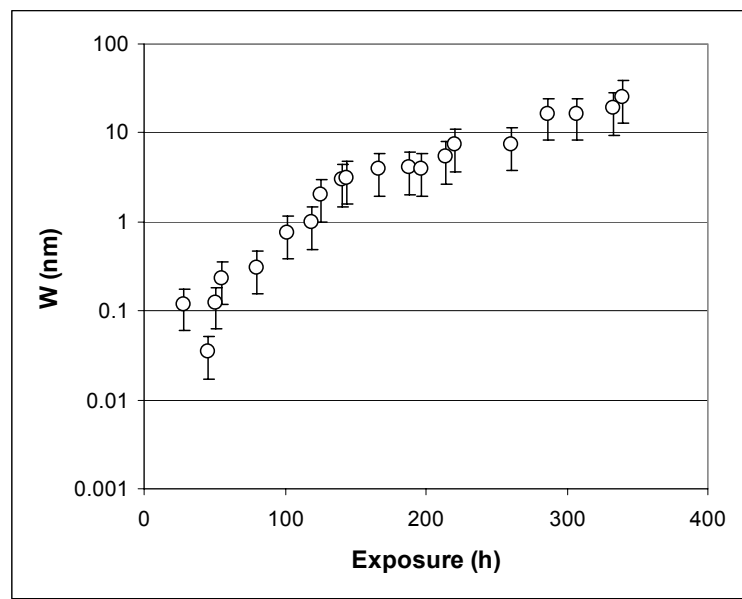

(a)

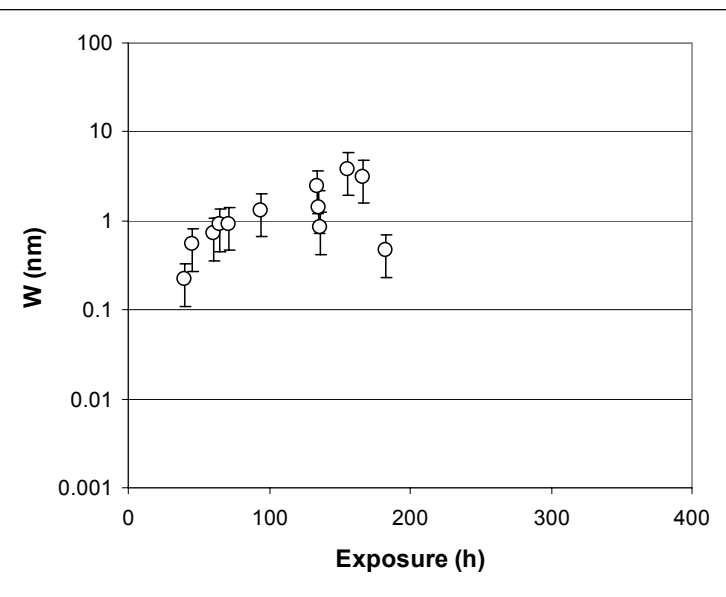

(b)

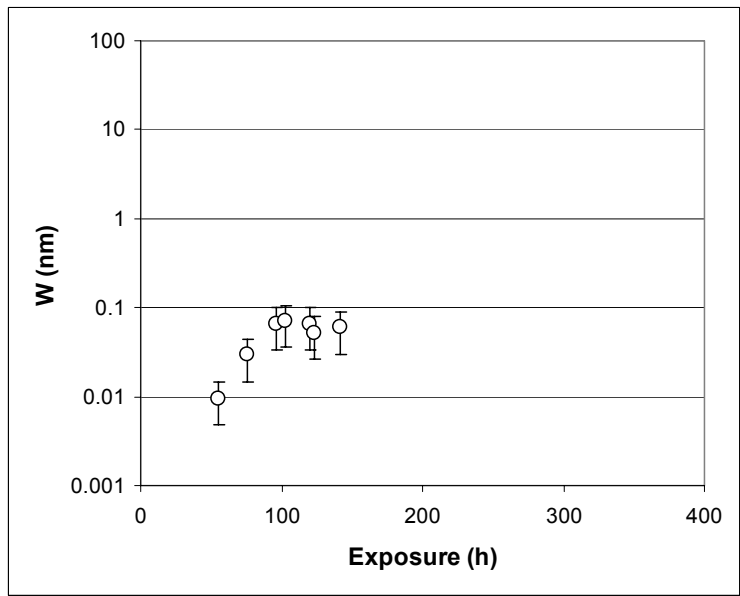

(c)

Figure 59. Oxide film (layer in which point defect transport occurs) growth on 347 stainless steel exposed to supercritical water. Concentrations of dissolved oxygen: (a) $-100 \mathrm{ppb}$, (b) $-400 \mathrm{ppb}$, and (c) -1400 $\mathrm{ppb}$.

In-situ monitoring of the film formation using controlled distance electrochemistry also allowed to obtain information on the strength of the electric field in the oxide (Figure 60) and electronic and ionic diffusion coefficients (Figure 61). At the present time, this is the only technique, which allows to obtaint key film parameters at the atomic/lattice level required for modeling oxidation of alloys and their lifetime using Point Defect Model or its various modifications (MCM model, in particular).

A decrease in the electric field strength with time was observed for low oxidizing conditions (Figure 60a). At the beginning of the exposure electric field strength falls in to the 
range of "high field approximation" and after 200-300 hours of exposure the electric field strength is clearly corresponds to "low field approximation" range. More oxidizing conditions tend to level off variation in the electric field strength with time (Figure $60 \mathrm{~b}$ and $\mathrm{c}$ ) and formed films are best described (and accordingly modeled) using "high field" approximation.

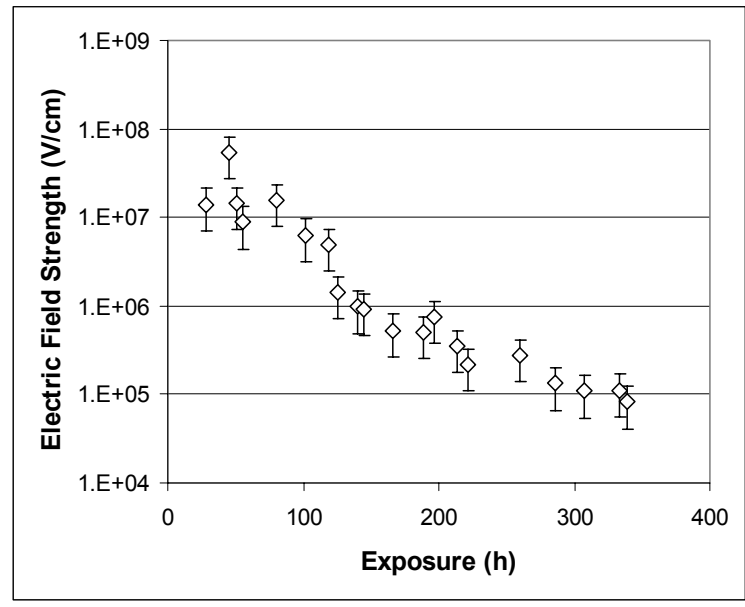

(a)

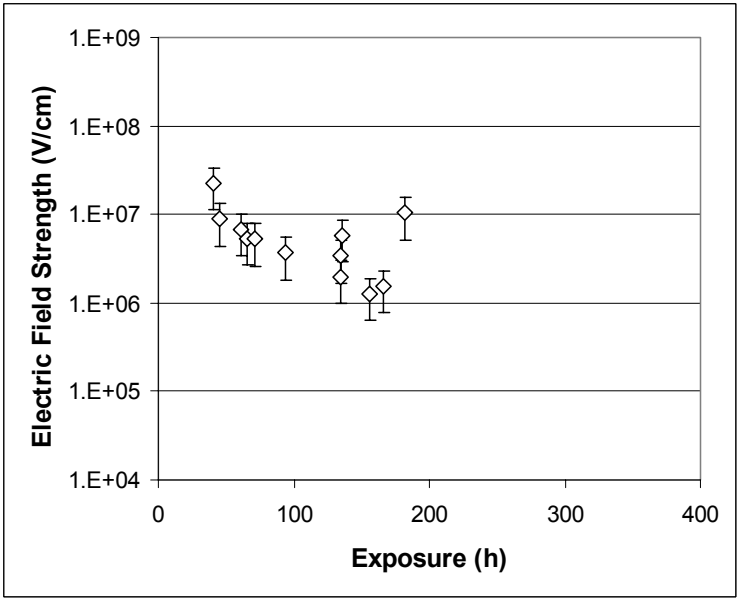

(b)

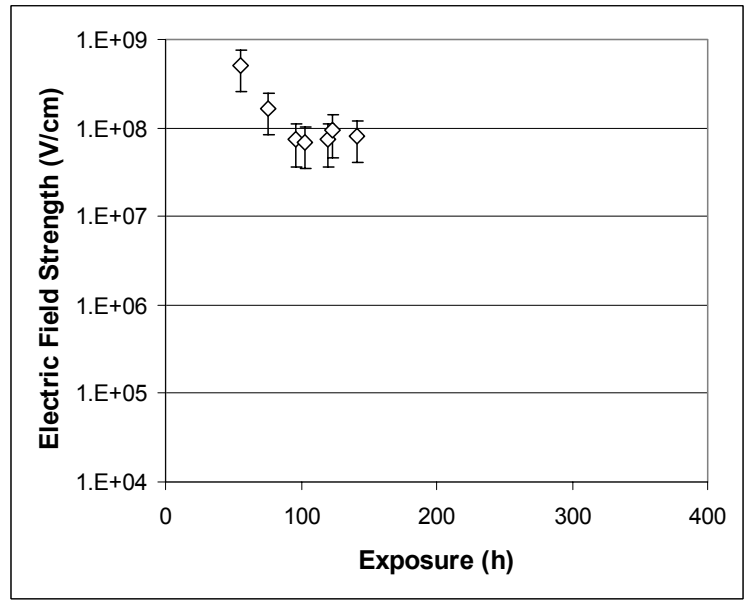

(c)

Figure 60. Strength of the electric field across the oxide film formed on 347 stainless steel exposed to supercritical water. Concentrations of dissolved oxygen: (a) $-100 \mathrm{ppb}$, (b) $-400 \mathrm{ppb}$, and (c) -1400 ppb. 
Data on diffusion coefficents derived form CDE measurements exibit distincltly different behaviour at low and high oxidizing conditions (Figure 61), which serves as an additional confiramtion of the earlier statement on "critical" dissolved oxygen levels. At low oxidizing conditions electronic and ionic diffusion coefficients are comparable (Figure 61a). Increase in the dissolved oxygen level above $\sim 400 \mathrm{ppb}$ results in (a) decrease in the magnitude of both diffusion coefficients and (b) electronic diffusion coefficients becom 2-3 orders of magnitude higher than ionic diffusion coefficients (Figure $61 \mathrm{~b}$ and c). Two different algorithms have been used to derive ionic diffusion coefficients.

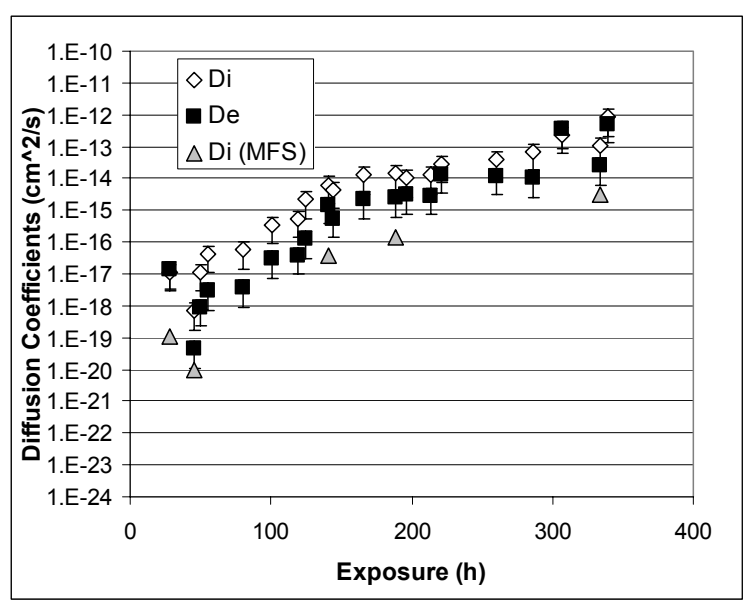

(a)

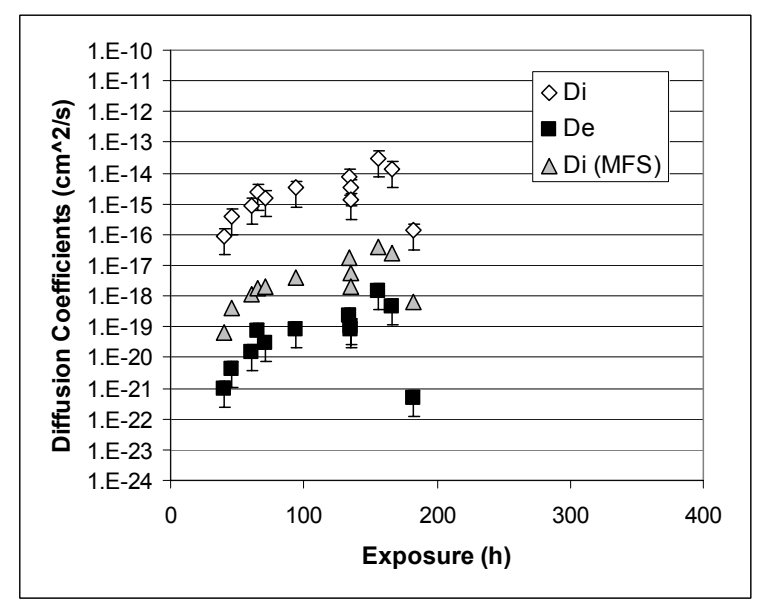

(b)

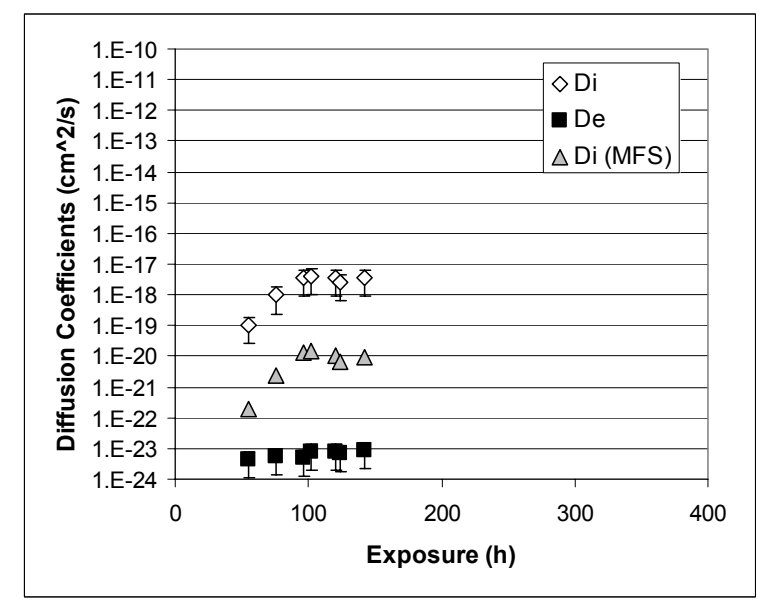

(c)

Figure 61. Ionic $\left(D_{i}\right)$ and electronic $\left(D_{e}\right)$ diffusion coefficients of the oxide film formed on 347 stainless steel exposed to supercritical water. Concentrations of dissolved oxygen: (a) $-100 \mathrm{ppb}$, (b) $-400 \mathrm{ppb}$, and (c) $-1400 \mathrm{ppb}$. 
Data obtained from impedance measurements in symmetrical configuration is labeled as "Di" and data obtained from impedance measurements in asymmetrical configuration is labeled and "Di (MFS)." In principle (or more exactly in case if impedance spectra are not noisy) both data should be close (as in the case of low dissolved oxygen concentraion in Figure 61a.) Discrepances observed for the higher oxygen levels (Figure 61b and c) were ascribed to hihger level of noise in the measured spectra and should be treated as an uncertainty in the derived ionic diffusion coefficients.

It can be concluded that both charge transfer and ionic point defect transport, i.e. a Warburg-like impedance, plays an important role in the electrochemical properties of oxide films formed on stainless steels in supercritical water. The values of the charge transfer and Warburg impedance decrease with increase in dissolved oxygen concentrations indicating that more defective (or less stable) films are being formed. Effect of oxidizing conditions on charge transfer resistance is more pronounced at high oxygen levels in comparison with diffusion resistance.

\section{Oxide film transport properties for pure nickel}

It is worth mentioning that the reproducibility of the electrochemical measurements on Ni-based alloys used in this work is not as good as on Fe-based materials at high temperatures. Oxide film characterization for pure nickel was performed for three levels of dissolved oxygen: 100,500 , and $2500 \mathrm{ppb}$. Electronic conductivity of the oxide films grown at $500 \mathrm{ppb}$ and 2500 $\mathrm{ppb}$ is essentially the same and about 2 times higher than for the films grown under $100 \mathrm{ppb}$ of dissolved oxygen (Figure 62). In most cases oxide film growth was almost instantaneous in comparison with stainless steel and the magnitude of the contact electrical resistance was 1-2 orders of magnitude higher. Evolution of the measured impedance spectra reveals rapid formation of a film with a charge transfer process (characteristic frequency about $1 \mathrm{MHz}$ ) and a slow diffusion process with characteristic frequency about $10 \mathrm{~Hz}$ (Figure 63). Impact of oxidizing conditions on impedance parameters of the film was found to be low (Figure 64). As shown in Figure 64 diffusion resistance of the films formed under all levels of dissolved oxygen are comparable and they do not change with exposure time. The same is true and for charge transfer resistance. Measured data on Young's impedance (Figure 65) are noisy and allowed only semi-quantitative conclusions that (1) the thickness of the mixed conducting layer is in the order of $1000 \mathrm{~nm}$ for all cases and this thickness does not seem to increase with exposure time (Figure 66) and (2) the strength of the electric field in the film corresponds to the "low field" range (Figure 67). Ionic diffusion coefficients are significantly higher than electronic diffusion coefficients for all oxidizing conditions explored (Figure 68). 


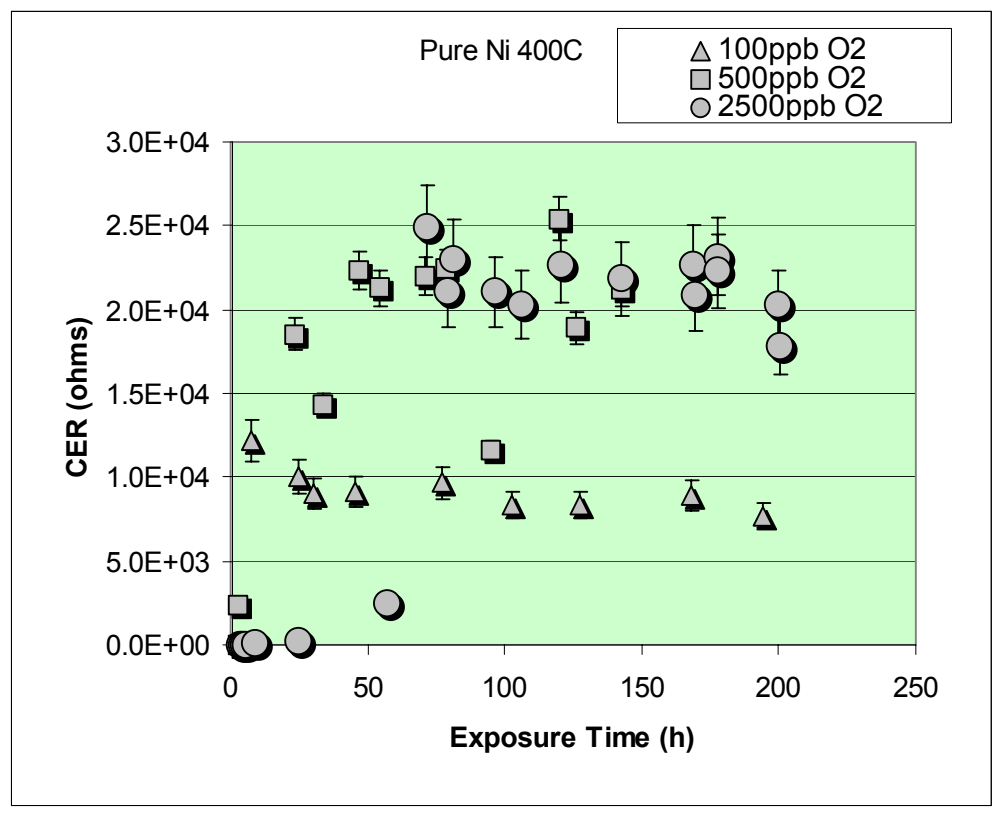

Figure 62. Electronic properties of the oxide film formed on pure nickel in supercritical water at various levels of dissolved oxygen.

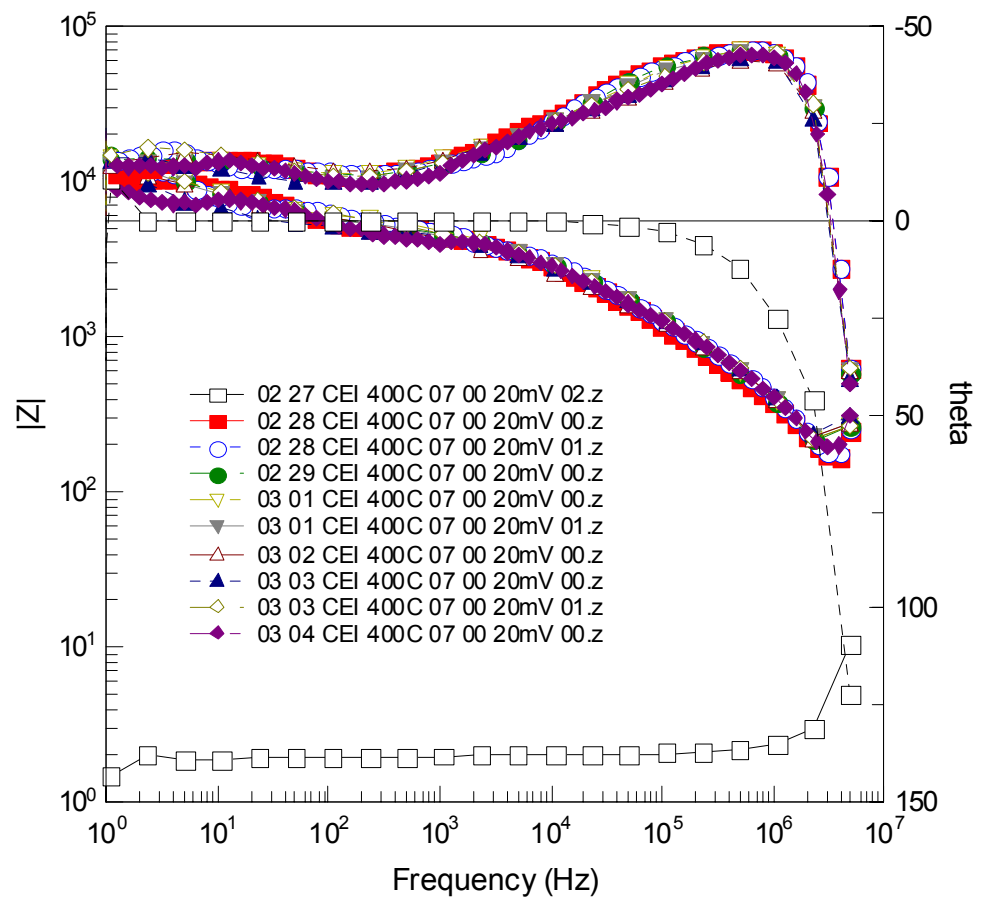

Figure 63 Evolution of the impedance spectra for the oxide film growing on pure nickel in supercritical water with $500 \mathrm{ppb}$ of dissolved oxygen. 


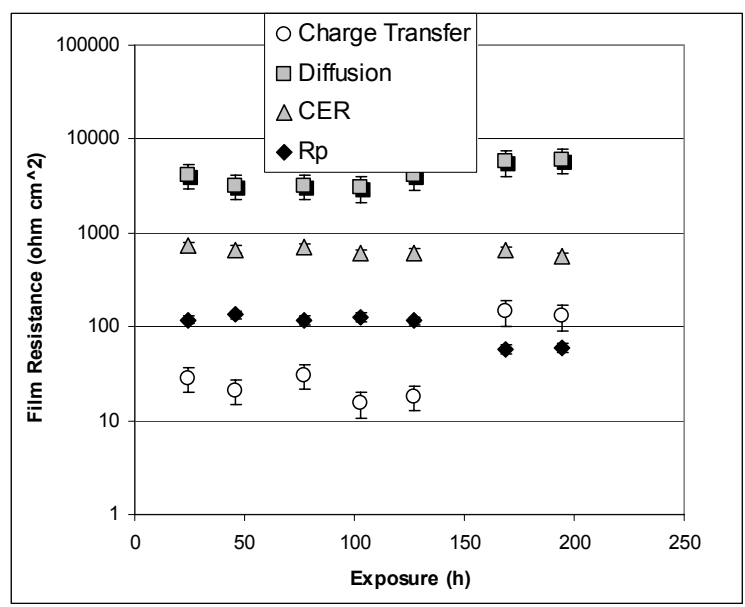

(a)

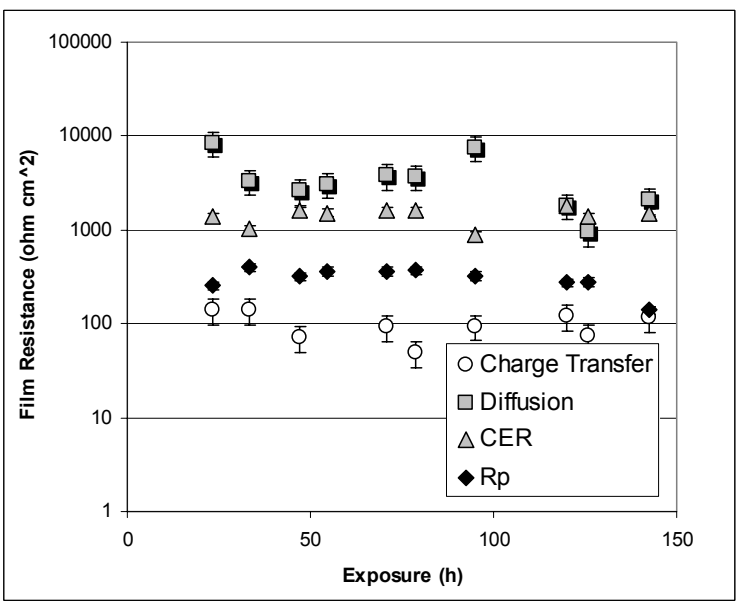

(b)

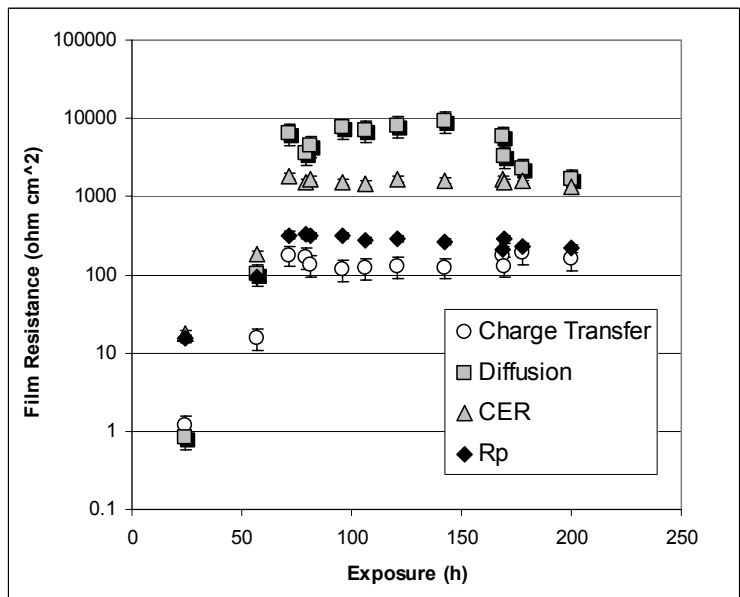

(c)

Figure 64. Impedance parameters of the oxide film formed on pure nickel exposed to supercritical water: charge transfer resistance, diffusion (Warburg) resistance, contact electric resistance (CER) and polarization resistance $\left(R_{p}\right)$. Concentrations of dissolved oxygen: (a) - $100 \mathrm{ppb}$, (b) - $500 \mathrm{ppb}$, and (c) $2000 \mathrm{ppb}$. 


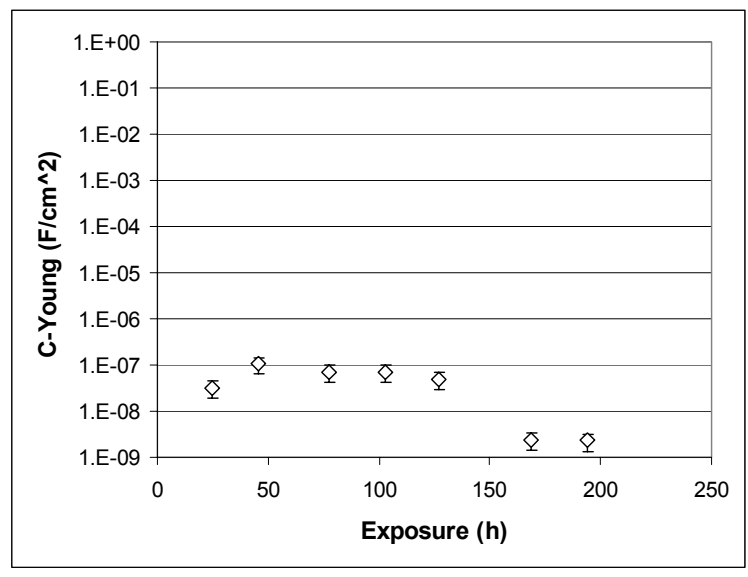

(a)

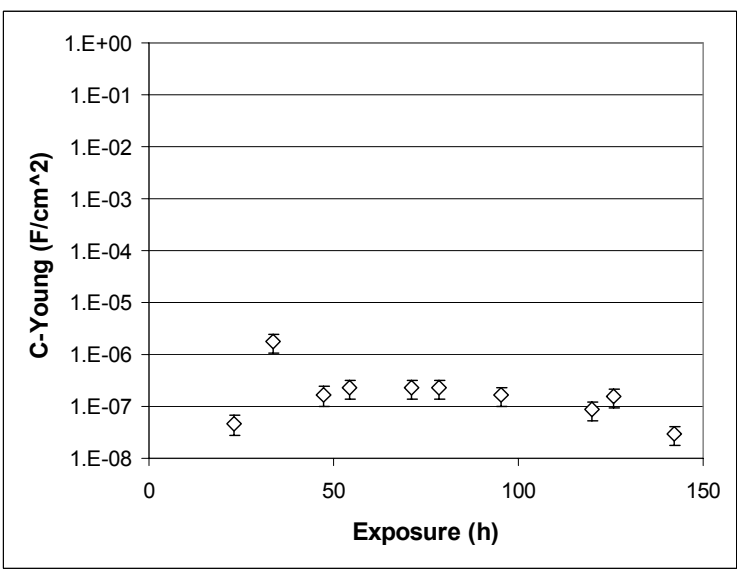

(b)

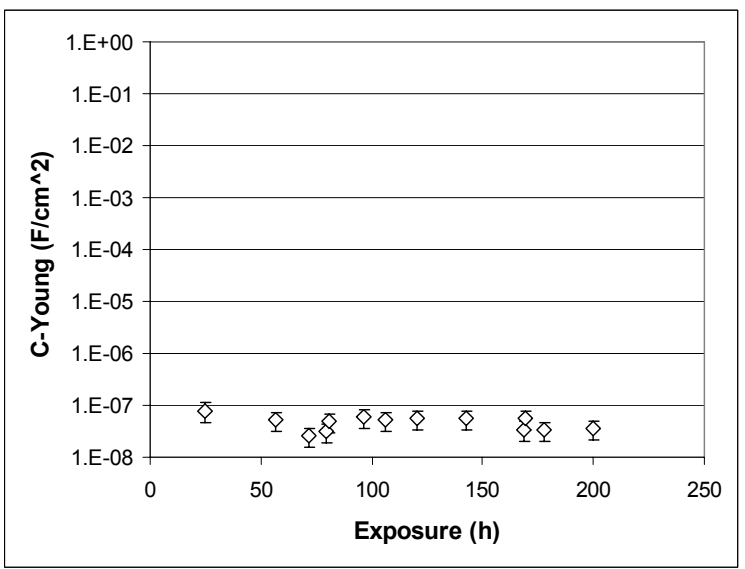

(c)

Figure 65. Effect of supercritical water oxidizing conditions on Young's impedance of the oxide film growing on pure nickel. Concentrations of dissolved oxygen: (a) $-100 \mathrm{ppb}$, (b) $-500 \mathrm{ppb}$, and (c) -2000 ppb. 


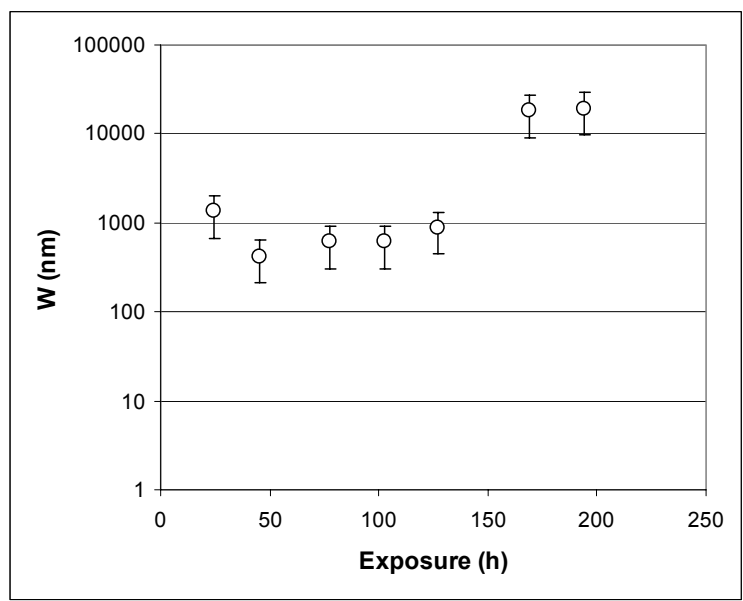

(a)

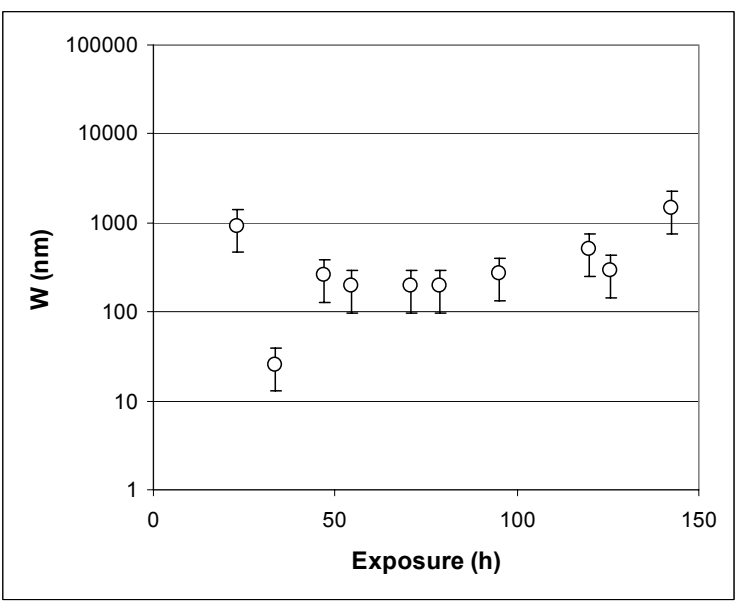

(b)

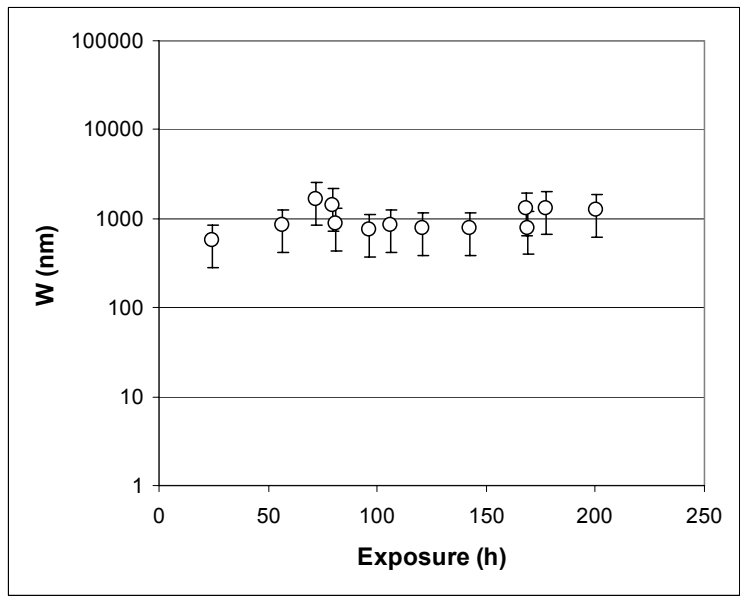

(c)

Figure 66. Oxide film (layer in which point defect transport occurs) growth on pure nickel exposed to supercritical water. Concentrations of dissolved oxygen: (a) $-100 \mathrm{ppb}$, (b) $-500 \mathrm{ppb}$, and (c) -2000 ppb. 


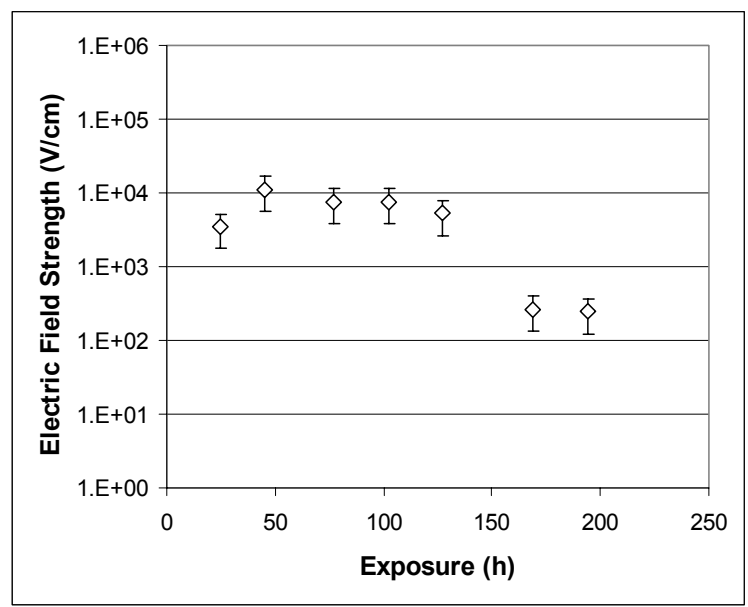

(a)

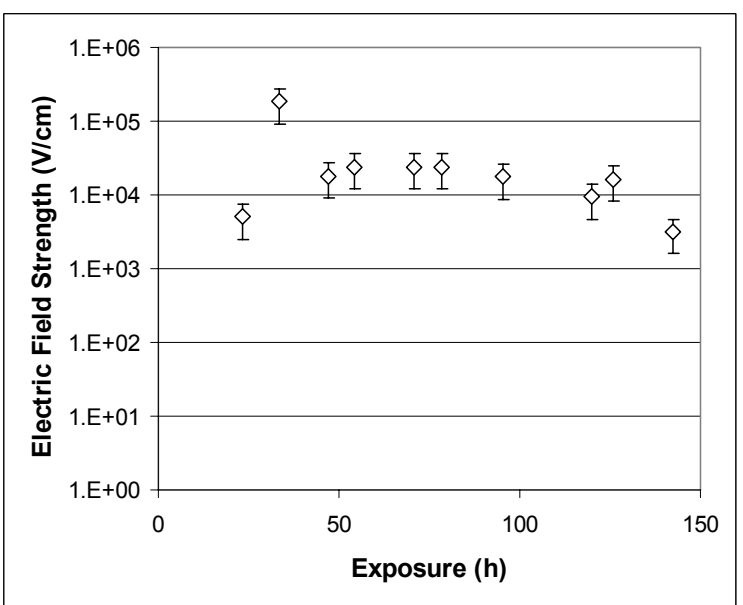

(b)

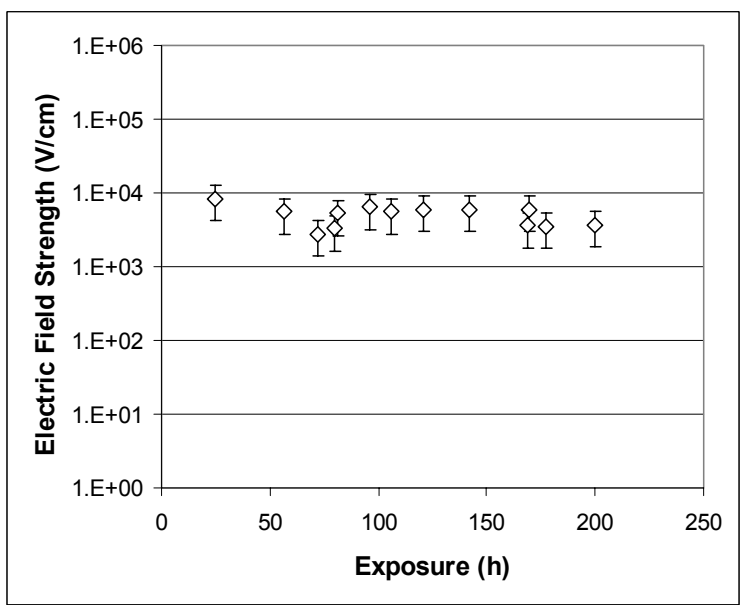

(c)

Figure 67. Strength of the electric field across the oxide film formed on pure nickel exposed to supercritical water. Concentrations of dissolved oxygen: (a) $-100 \mathrm{ppb}$, (b) $-500 \mathrm{ppb}$, and (c) -2000 $\mathrm{ppb}$. 

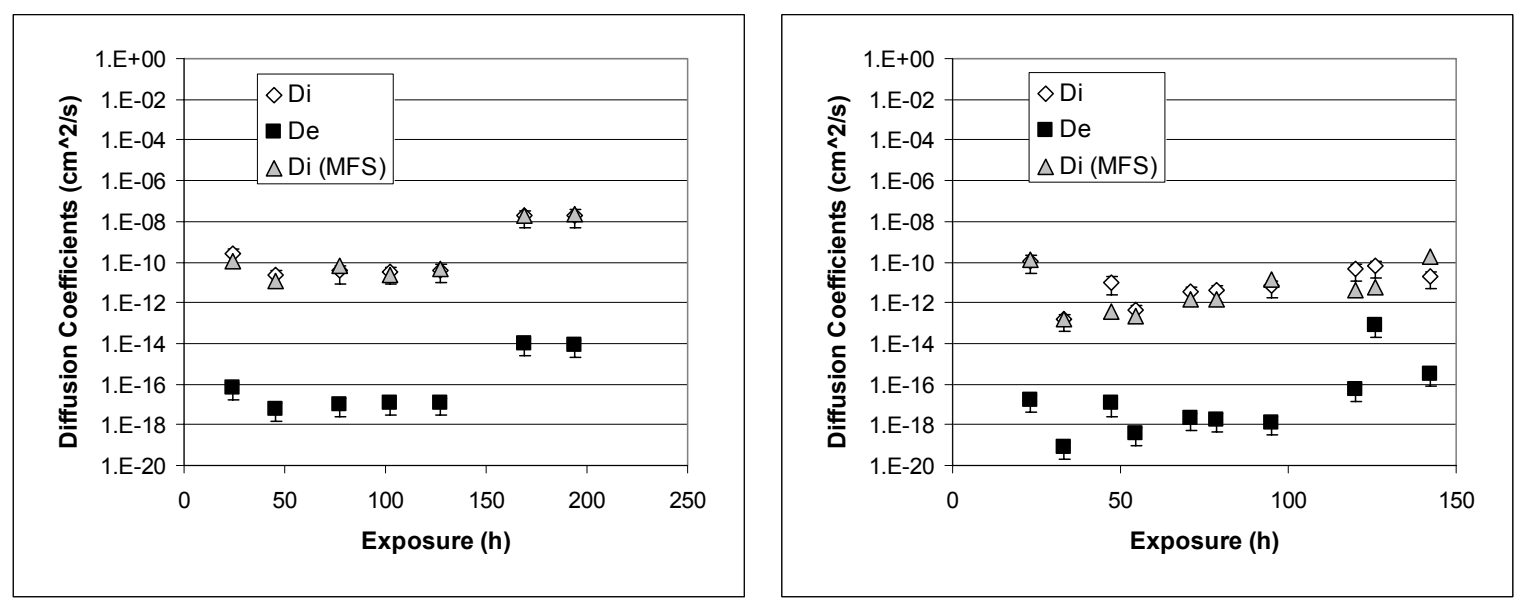

(a)

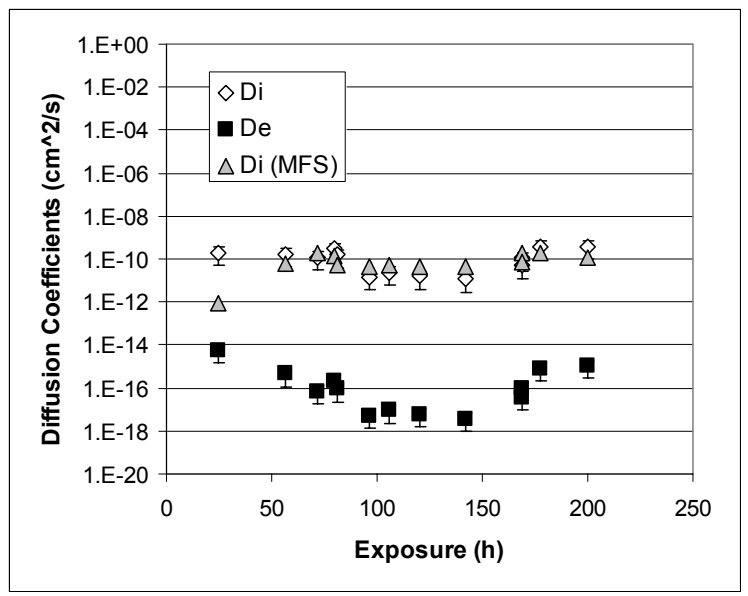

(c)

Figure 68. Ionic $\left(D_{i}\right)$ and electronic $\left(D_{e}\right)$ diffusion coefficients of the oxide film formed on pure nickel exposed to supercritical water. Concentrations of dissolved oxygen: (a) $-100 \mathrm{ppb}$, (b) $-500 \mathrm{ppb}$, and (c) $-2000 \mathrm{ppb}$. 


\section{Oxide film transport properties for nickel alloy 690}

Passivation behavior of A690 in supercritical water (Figure 69) was found to be significantly different for the one for pure nickel (Figure 62). Apparently oxidation behavior of nickel alloys in supercritical water is dominated by chromium content as in the case of subcritical water. CER measurements indicated that the oxide film forms slower on the A690 (Figure 69) than on stainless steel 347 (Figure 55), but electronic resistance of the film on the A690 is an order of magnitude higher. A combined effect of repassivation time and film stability on crack growth rate will be discussed later in the text.

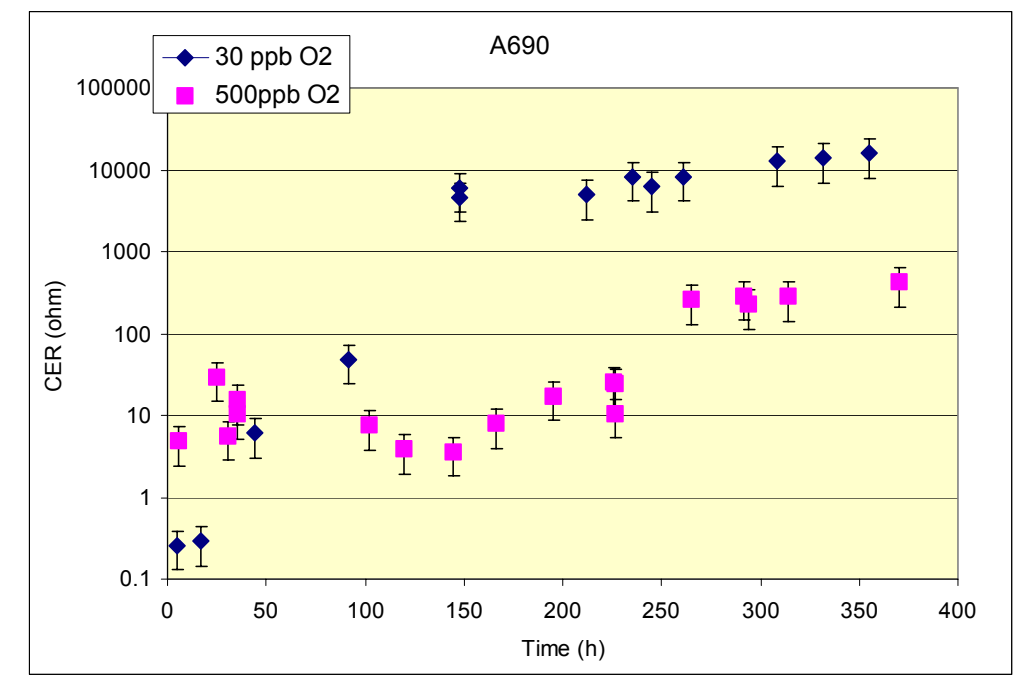

Figure 69. Electronic properties of the oxide film formed on alloy 690 in supercritical water at various levels of dissolved oxygen.

Evolution of the measured impedance spectra reveals slow formation of a film with a charge transfer process and a diffusion process (Figure 70). Charge transfer resistance and diffusion resistance are comparable at the initial stage of film formation; diffusion resistance becomes an order of magnitude larger after prolonged (several hundreds of hours) exposure (Figure 71). Data on Young's impedance (Figure 72) are noisy and allow only a semiquantitative conclusions that: the thickness of a mixed conducting layer is in the order of tens on nano-meters (Figure 73) with electric field strength across it close to "high field" range (Figure 74) and ionic diffusion coefficients are 2 order of magnitude higher than electronic diffusion coefficients (Figure 75). 


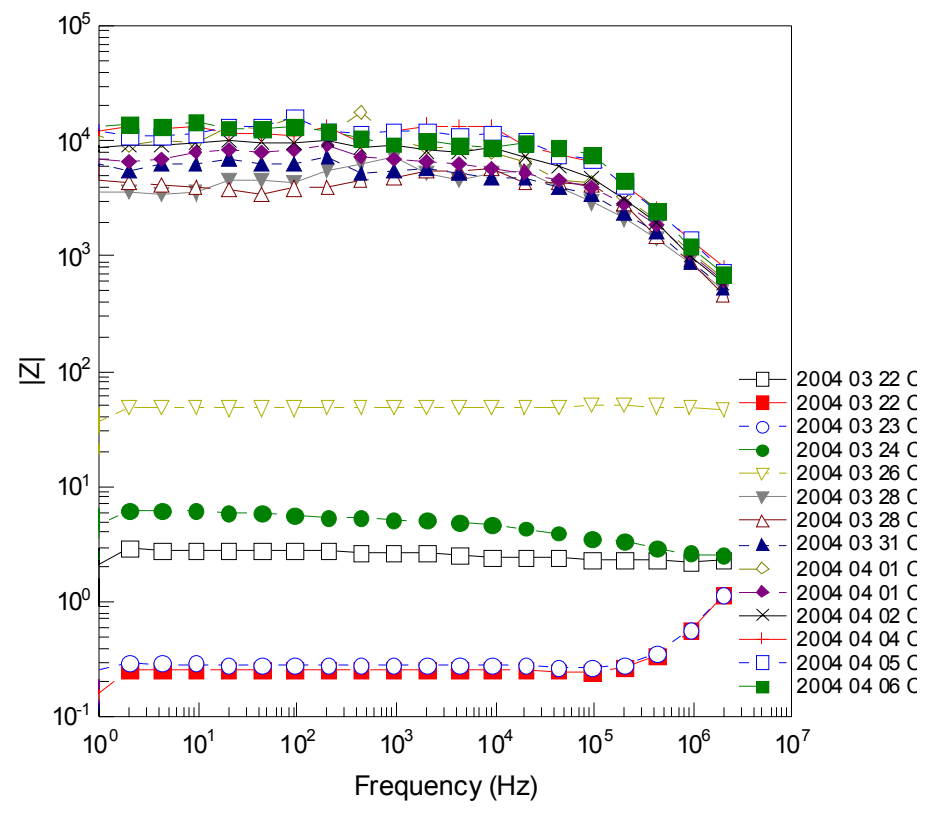

(a)

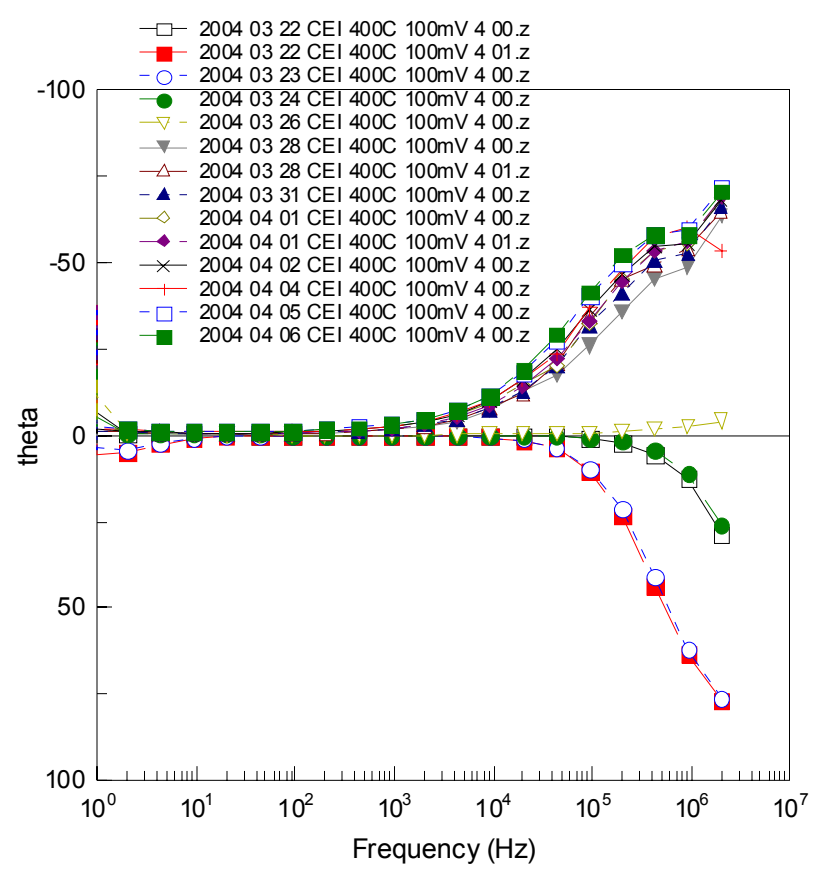

(b)

Figure 70. Evolution of the impedance spectra for the oxide film growing on A690 in supercritical water with $50 \mathrm{ppb}$ of dissolved oxygen: (a) - modulus of impedance, (b) - phase angle 


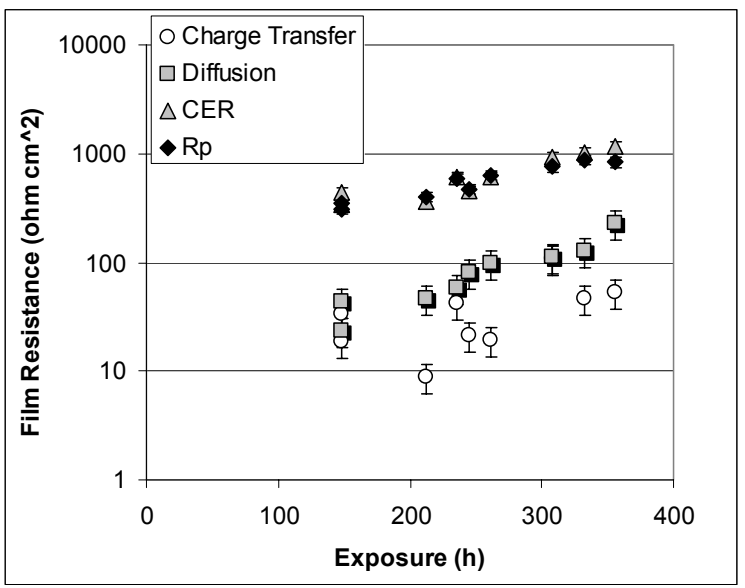

Figure 71. Impedance parameters of the oxide film formed on pure nickel exposed to supercritical water: charge transfer resistance, diffusion (Warburg) resistance, contact electric resistance (CER) and polarization resistance $\left(R_{p}\right)$. Concentration of dissolved oxygen $100 \mathrm{ppb}$

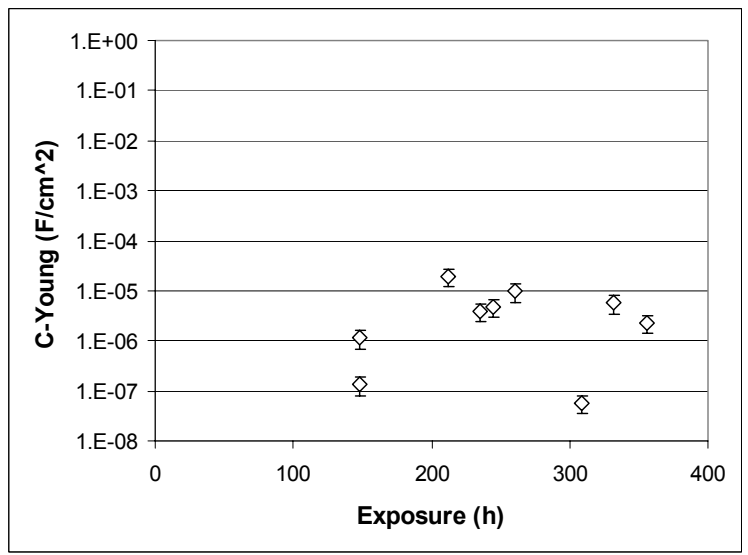

Figure 72. Evolution of Young's impedance of the oxide film growing on alloy 690 in supercritical water. Concentration of dissolved oxygen $100 \mathrm{ppb}$.

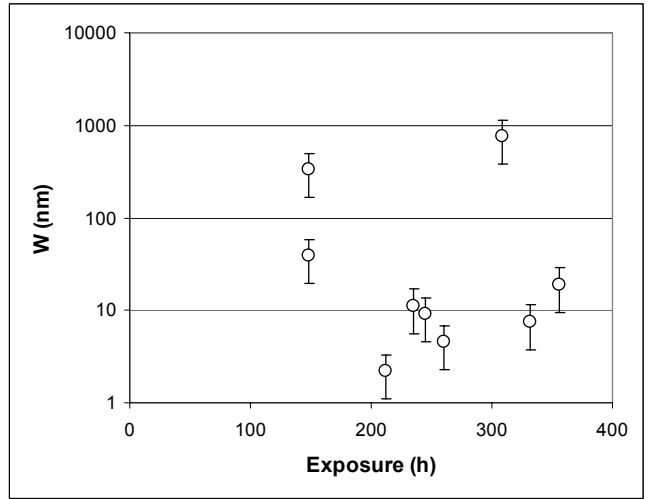

Figure 73. Oxide film (layer in which point defect transport occurs) growth on alloy 690 exposed to supercritical water. Concentration of dissolved oxygen $100 \mathrm{ppb}$. 


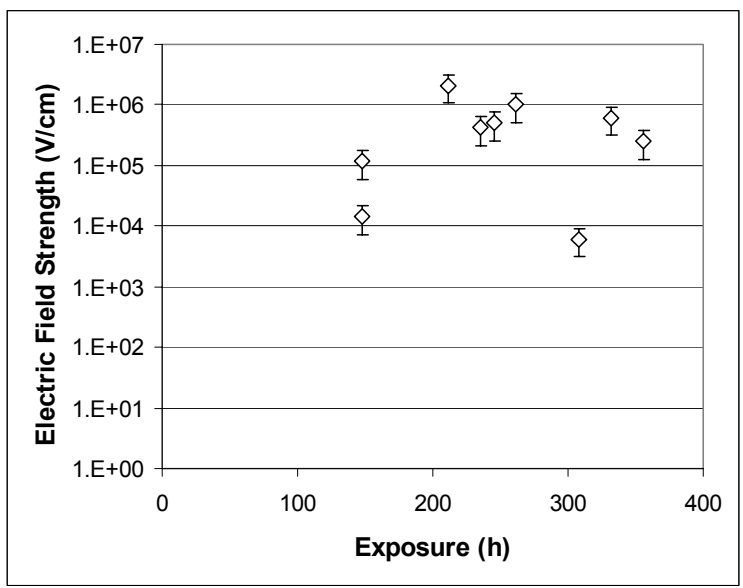

Figure 74. Strength of the electric field across the oxide film formed on alloy 690 exposed to supercritical water. Concentration of dissolved oxygen $100 \mathrm{ppb}$.

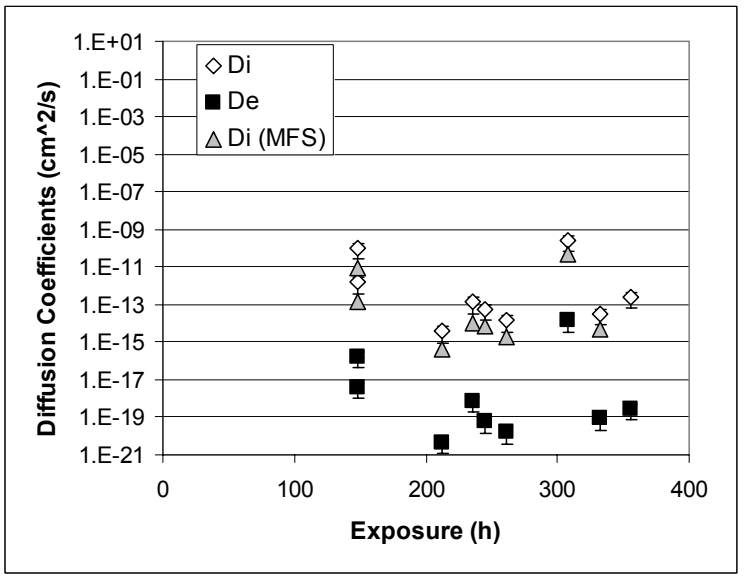

Figure 75. Ionic $\left(D_{i}\right)$ and electronic $\left(D_{e}\right)$ diffusion coefficients of the oxide film formed on alloy 690 exposed to supercritical water. Concentration of dissolved oxygen $100 \mathrm{ppb}$. 


\section{Oxide film transport properties for alloy T91}

Passivation behavior of alloy T91 in supercritical water $\left(400^{\circ} \mathrm{C}\right)$ was studied for two oxidizing conditions: $30 \mathrm{ppb}$ and $5000 \mathrm{ppb}$ of dissolved oxygen. The first case corresponds to advanced water chemistry conditions when radiolytically produces oxidizing species are suppressed by hydrogen injection (an analog of BWR hydrogen water chemistry). The second case simulates maximal conceivable oxidizing conditions that may exist in G-IV SCWR under neutron and gamma irradiation.

An oxide film formation was almost undetectable even after 350 hours of exposure. Electronic resistivity of the oxide film, as judged by CER measurements is extremely low (Figure 76). Time dependence of CER measured film resistivity suggests that a pre-existing film gradually dissolves under low dissolve oxygen concentrations (case for $30 \mathrm{ppb} \mathrm{O}_{2}$ in Figure 76). Dissolution of a pre-existing films was also observed and for highly oxidizing conditions (case for $5000 \mathrm{ppb} \mathrm{O}_{2}$ in Figure 76) during first 50 hours of exposure. Continued exposure of T91 to supercritical water with $5000 \mathrm{ppb} \mathrm{O}_{2}$ resulted in slow formation of new oxide film (Figure 76). These observations are in accord with studies performed by other authors at sub-critical temperatures.

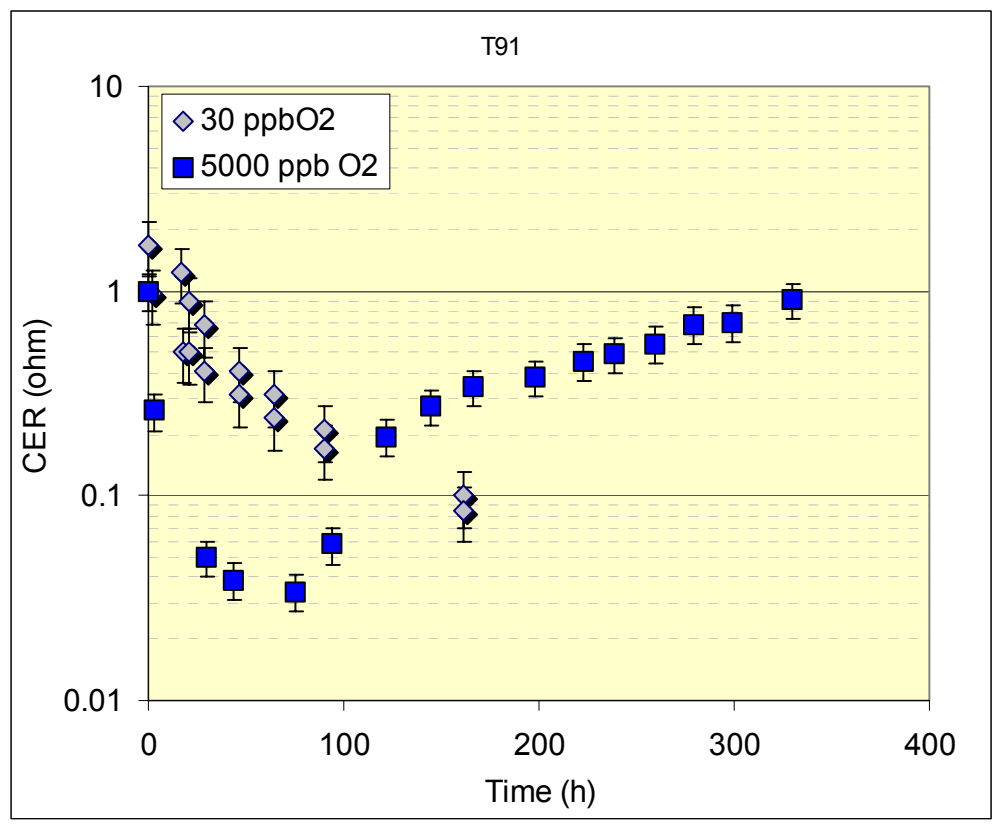

Figure 76. Electronic properties of the oxide film formed on alloy T91 in supercritical water at various levels of dissolved oxygen 
CEI impedance spectra monitored during 350 hours of exposure for highly oxidizing conditions (Figure 77) and for deeply de-oxygenated supercritical water (Figure 78) confirm that alloy T91 does not form a discernable oxide film at these conditions. In both cases impedance spectra reveal only inductive-ohmic behavior of the metal $/$ film $/$ metal system, which resembles the case of a bare metal. No capacitive, charge transfer or diffusion related time constants have been observed. Accordingly no derivation of the film's kinetic parameters has been undertaken. It should be mentioned parenthetically, that the absence of the oxide film resistant to charge and mass transfer between alloy T91 and supercritical water does not ultimately mean high susceptibility of the this alloy to SCC. Possible reasons for that are discussed further in the text.

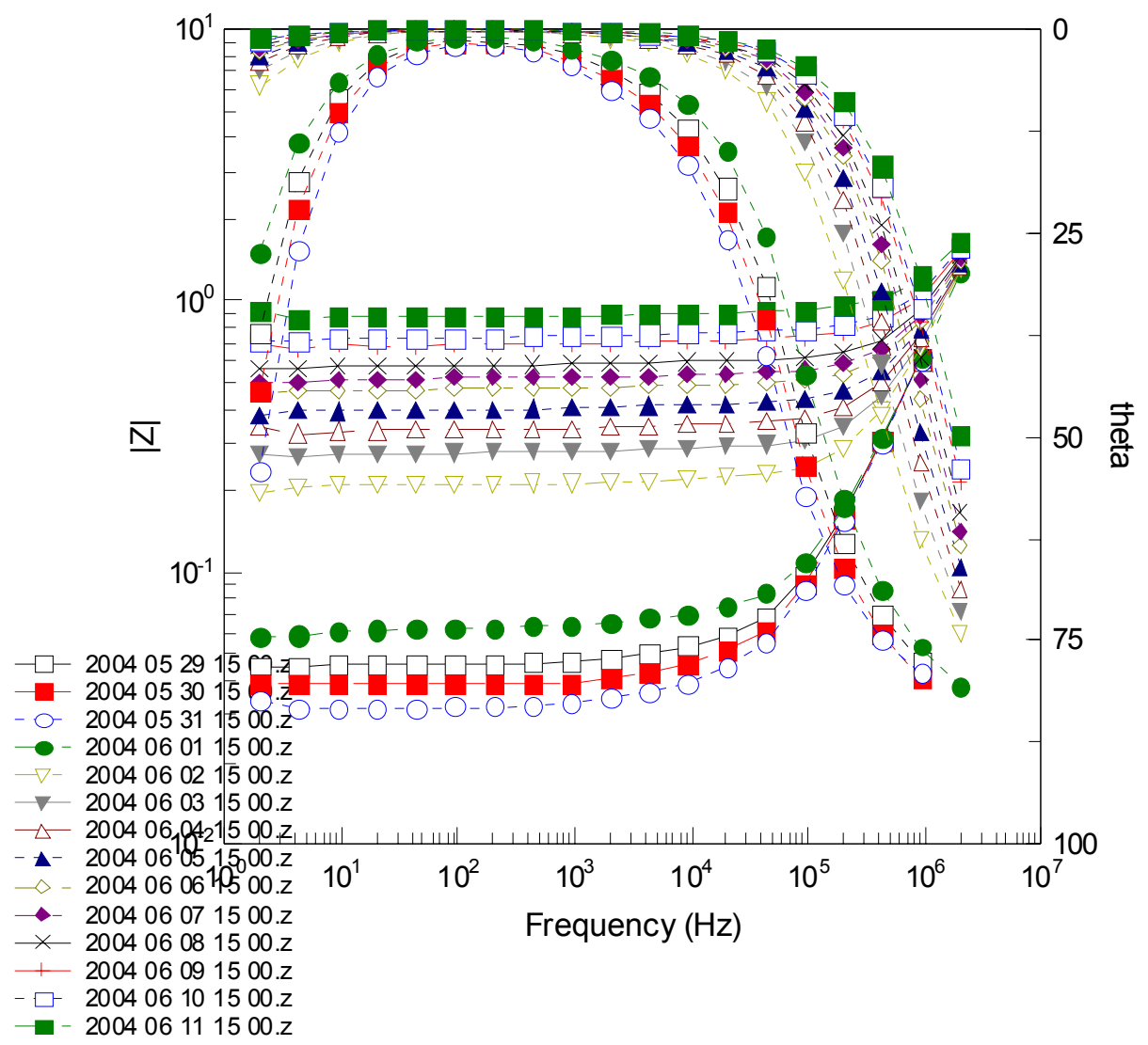

Figure 77. Evolution of CEI spectrum for oxide film growing on alloy T91 exposed to supercritical water with $5000 \mathrm{ppb}$ of dissolved oxygen. 


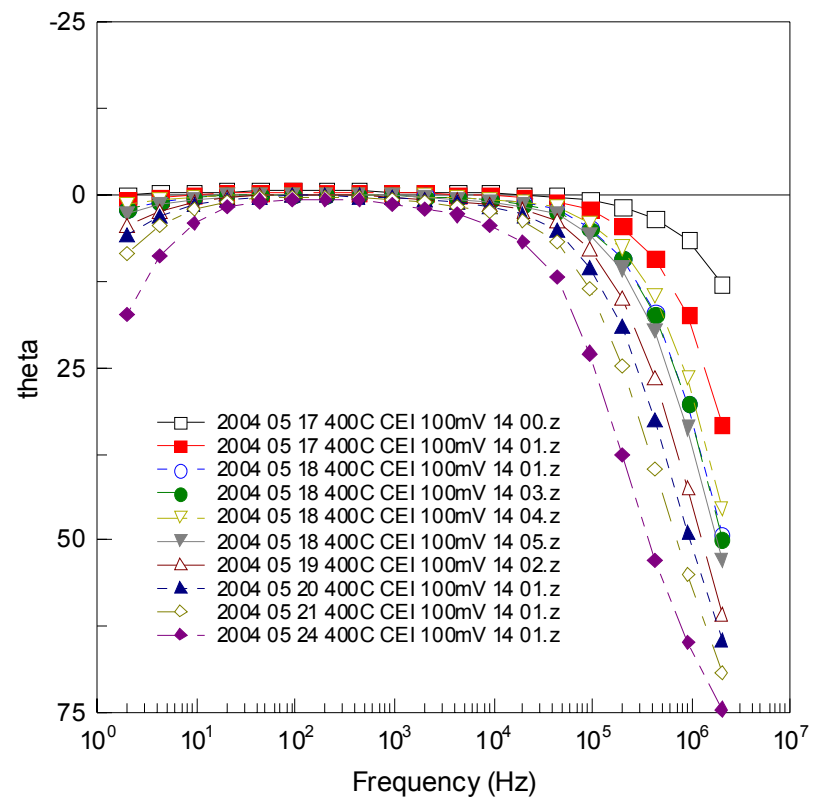

(a)

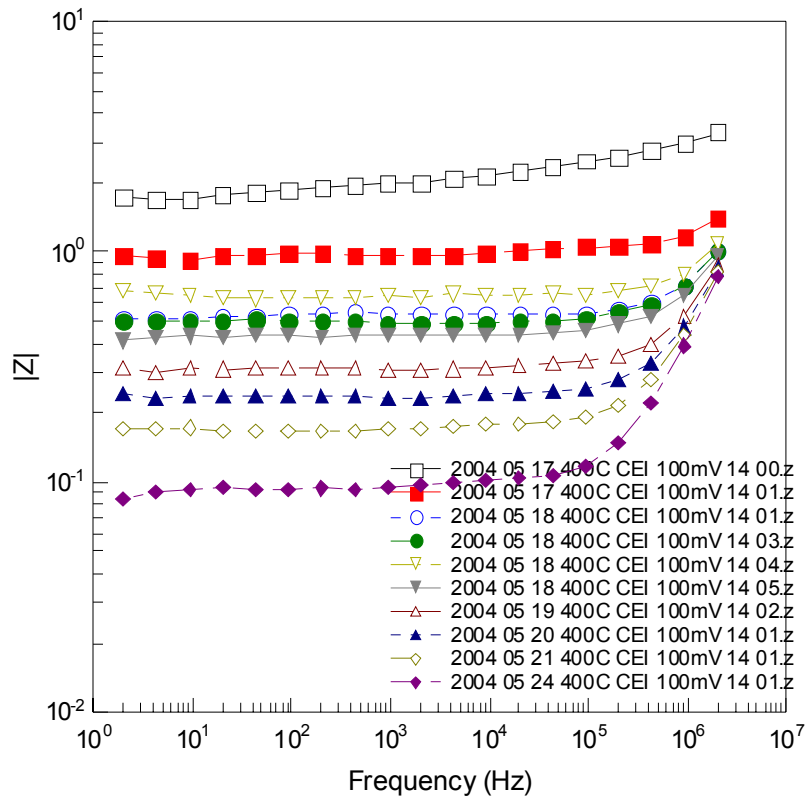

(b)

Figure 78. Evolution of CEI spectrum for oxide film growing on alloy T91 exposed to supercritical water with $30 \mathrm{ppb}$ of dissolved oxygen: (a) - phase angle, (b) - modulus 


\section{Oxide film transport properties for alloy HT9}

Passivation behavior of alloy HT9 in supercritical water $\left(400^{\circ} \mathrm{C}\right)$ was studied for two oxidizing conditions: $50 \mathrm{ppb}$ and 5-8 ppm of dissolved oxygen. As for T91, the first case corresponds to advanced water chemistry with suppressed radiolytically produced oxidizing species and the second case simulates maximal conceivable oxidizing conditions in SCWR heat transport circuit.

An oxide film formation was also almost undetectable for HT9 after $~ 300$ hours of exposure. While electronic resistivity of the oxide film formed on HT9 (Figure 79) seems to be an order of magnitude higher than for T91 (Figure 76) measured data exhibit fluctuations, which are on the same order of magnitude as film resistivity changes due to variation in dissolved oxygen level from $50 \mathrm{ppb}$ to $5 \mathrm{ppm}$. Exposure of HT9 to supercritical water with high oxygen concentration $(5 \mathrm{ppm})$ resulted in a noticeable monotonic growth of the CER measured film electronic resistivity during first 200 hours of exposure. Unlike the case for T91, no regular time dependence of CER measured film resistivity was observed for HT9 during exposure to deeply de-oxygenated ( $50 \mathrm{ppm}$ of dissolved $\mathrm{O}_{2}$ ) supercritical water.

CEI impedance spectra monitored during 300 hours of exposure for highly oxidizing conditions (Figure 80 and Figure 81 ) and for deeply de-oxygenated supercritical water (Figure 82) confirm that alloy HT9 does not form a discernable oxide film at these conditions. In both cases impedance spectra reveal only inductive-ohmic behavior of the metal/film/metal system, which resembles the case of a bare metal. No capacitive, charge transfer or diffusion related time constants have been observed. However, some indication to formation of high frequency time constant (possible charge-transfer related) after 200 hours of exposure (Figure 81) may be a subject for a more detailed examination. Analysis of TLEC spectra (Figure 83 and Figure 84) led to a conclusion that both high and low frequency time constants are associated with thin layer of supercritical water between CDE samples and that they contain no information on oxide film properties. As in the case of T91 no derivation of the film's kinetic parameters has been undertaken.

These observations are in accord with studies performed by other authors at sub-critical temperatures. As in the case for T91, it should be mentioned again, that the absence of the oxide

film resistant to charge and mass transfer between alloy HT9 and supercritical water does not ultimately mean high susceptibility of the this alloy to SCC. 


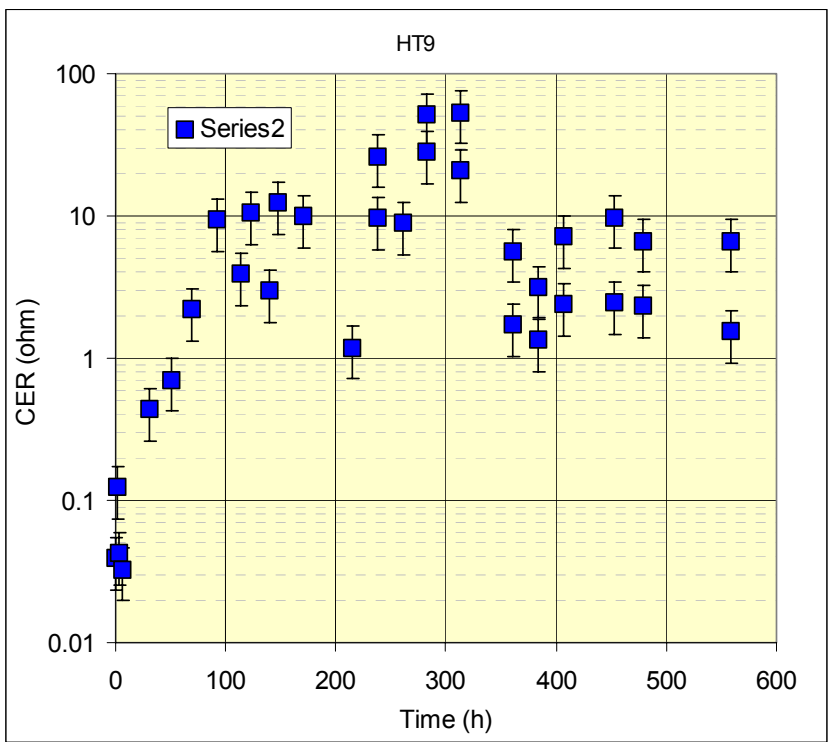

(a)

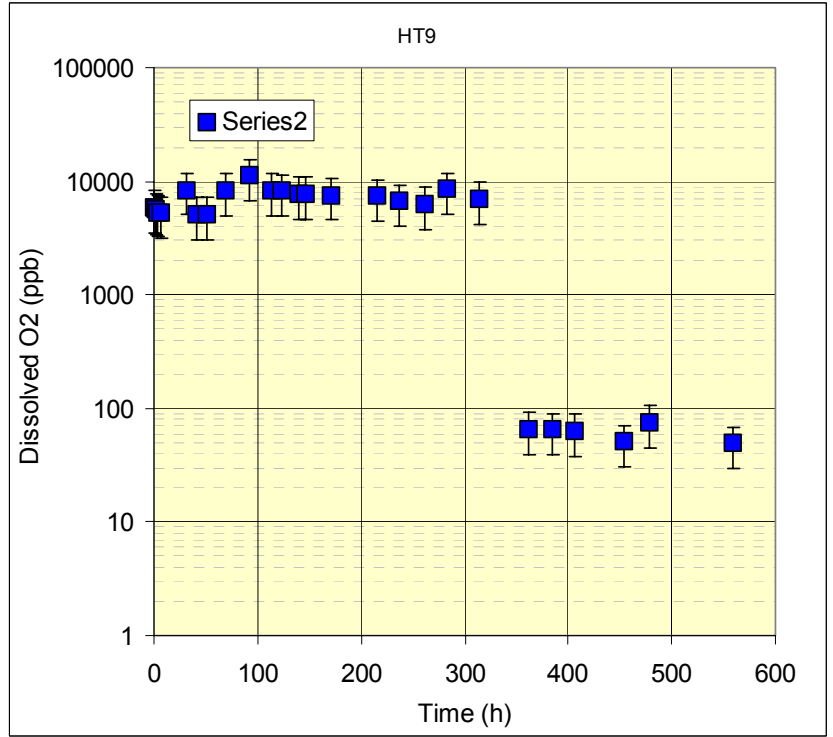

(b)

Figure 79. Electronic properties of the oxide film formed on alloy T91 in supercritical water (a) at various levels of dissolved oxygen (b) 


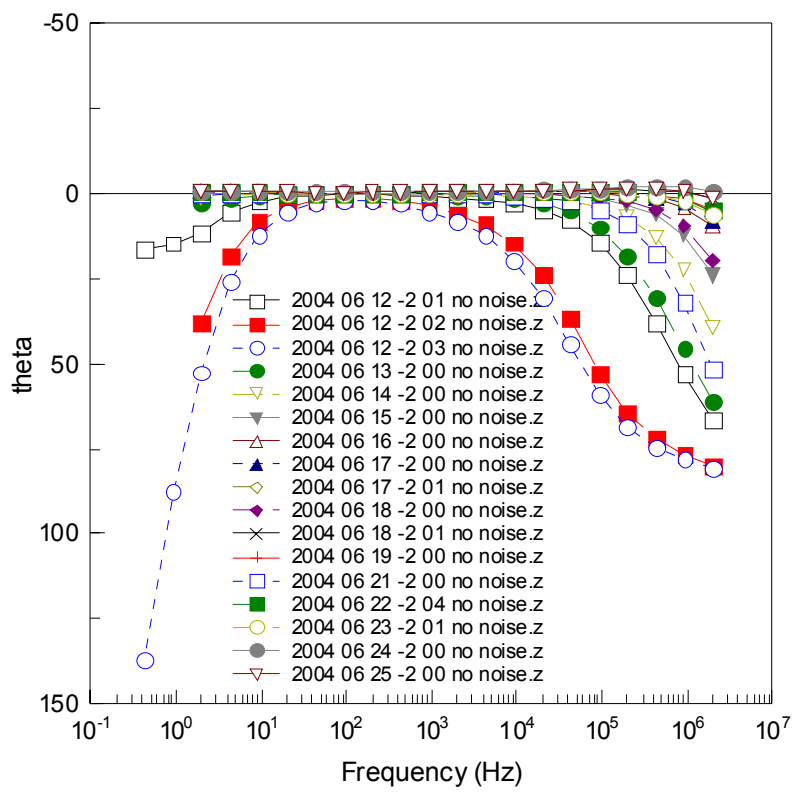

(a)

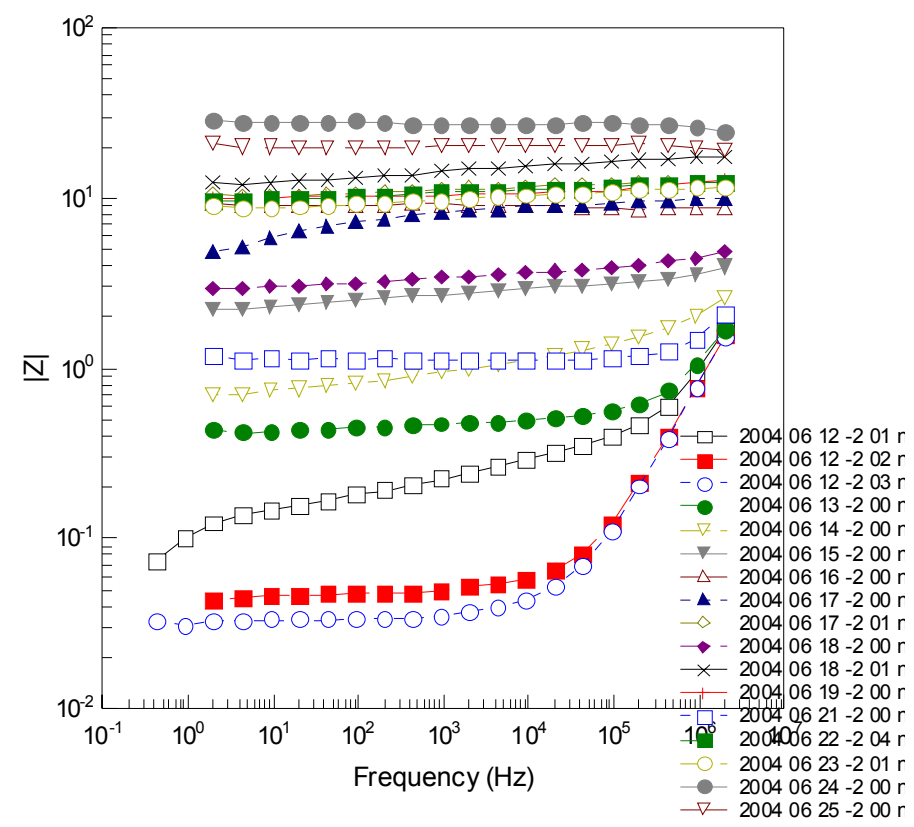

(b)

Figure 80. Evolution of CEI spectrum for oxide film growing on alloy HT9 exposed to supercritical water with 5000 ppb of dissolved oxygen: (a) - phase angle, (b) - modulus 


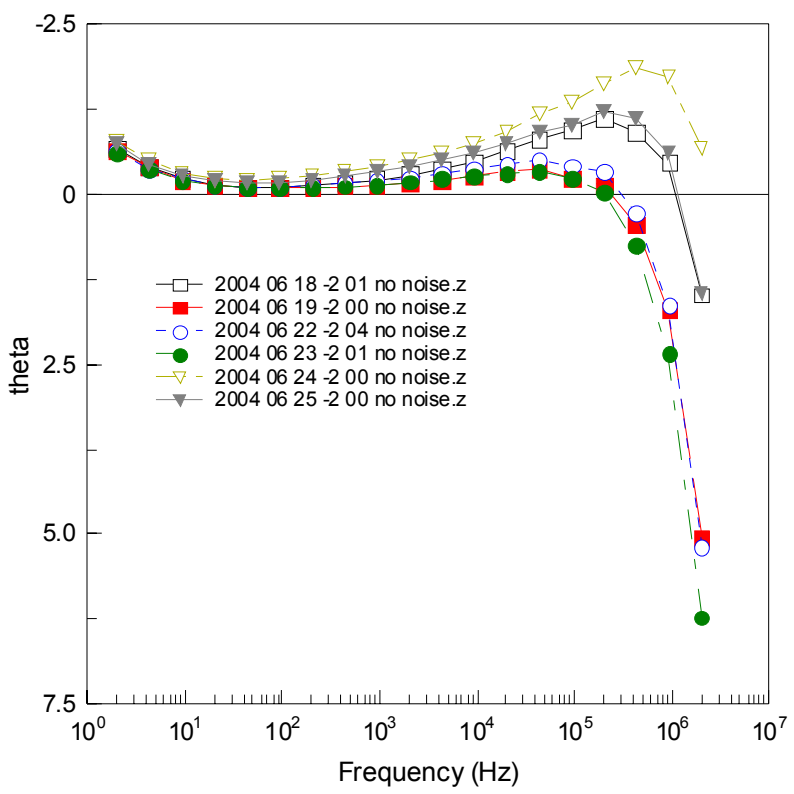

(a)

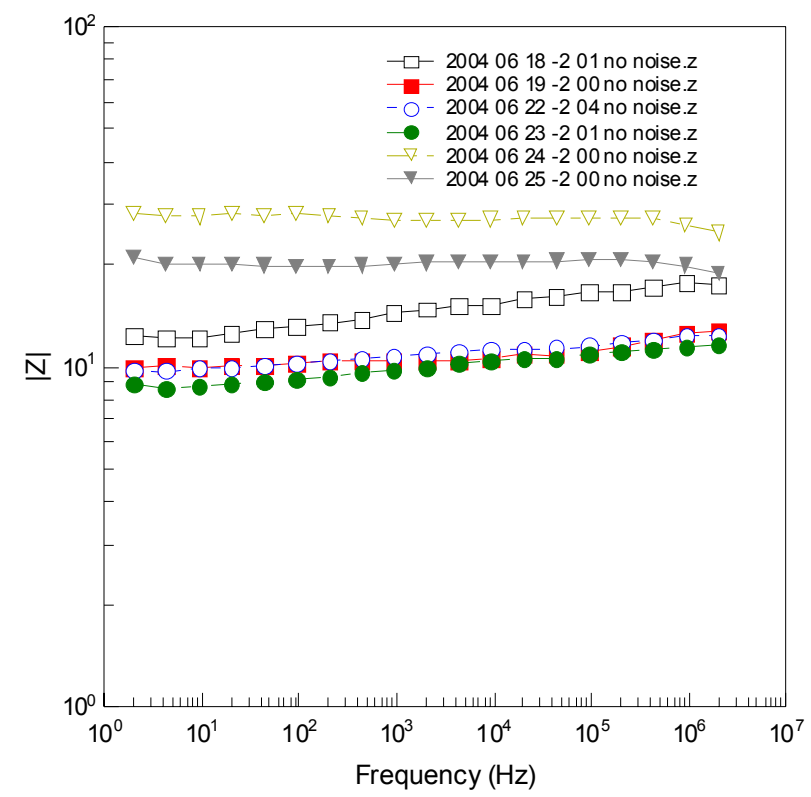

(b)

Figure 81. Evolution of CEI spectrum for oxide film growing on alloy HT9 exposed to supercritical water with 5000 ppb of dissolved oxygen: (a) - phase angle, (b) - modulus. Data from Figure 80 for longer exposure times only 


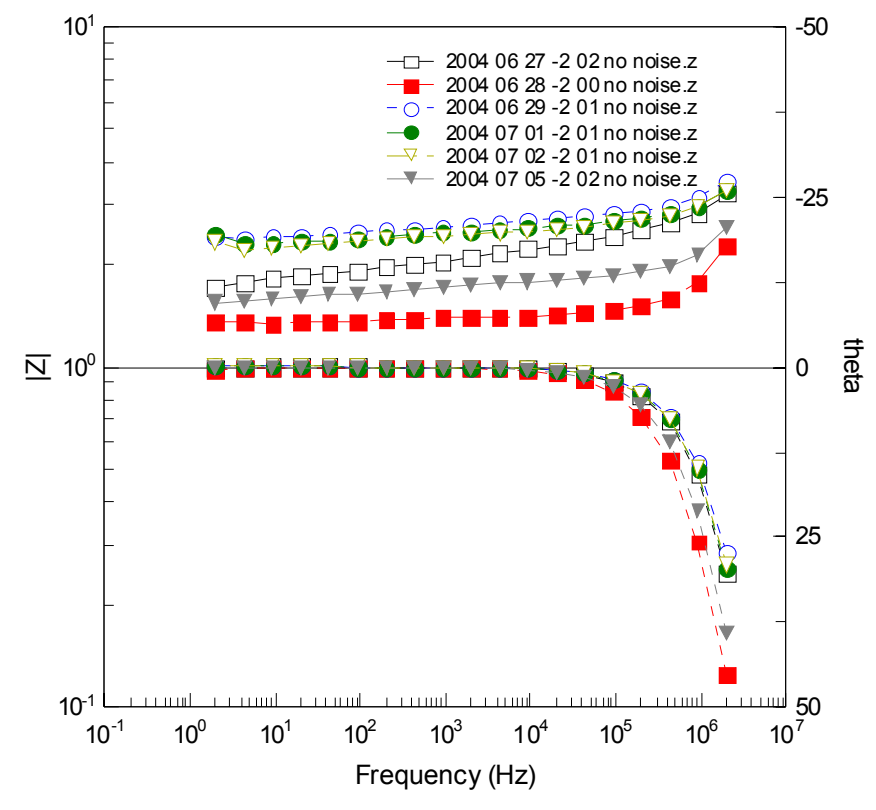

(a)

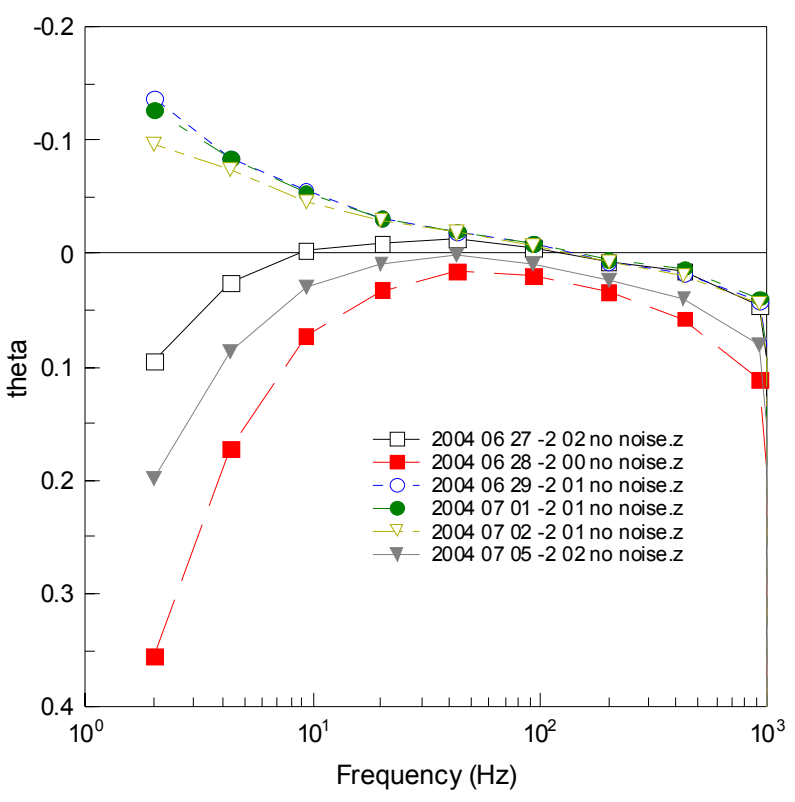

(b)

Figure 82. Evolution of CEI spectrum for oxide film growing on alloy HT9 exposed to supercritical water with $50 \mathrm{ppb}$ of dissolved oxygen: (a) -modulus and phase angle, (b) - phase angle for frequency $<1 \mathrm{kHz}$. 


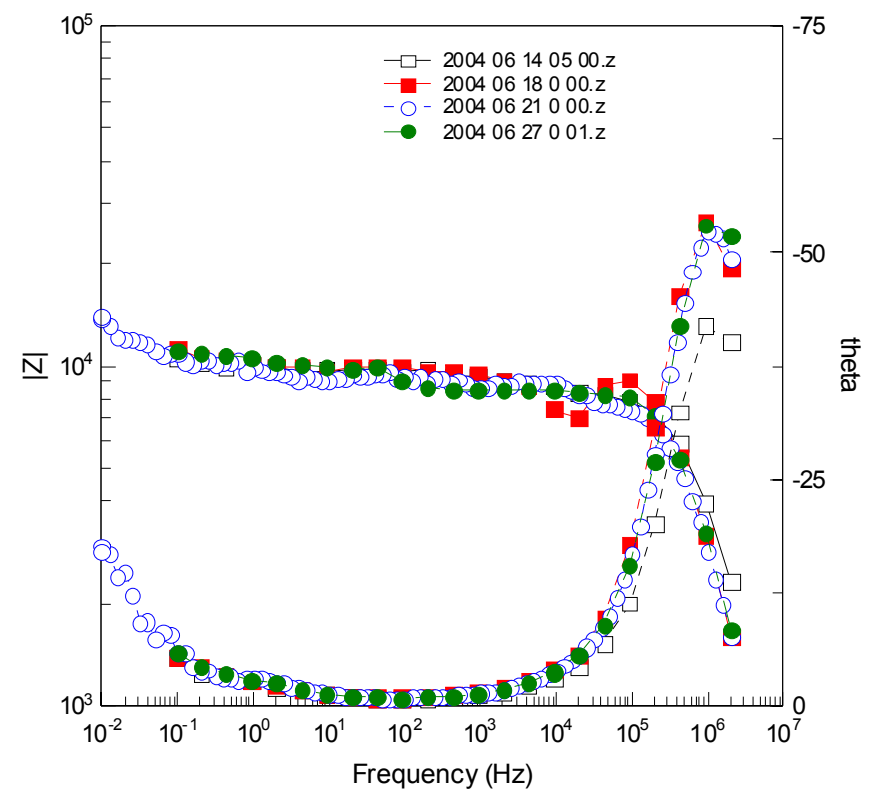

(a)

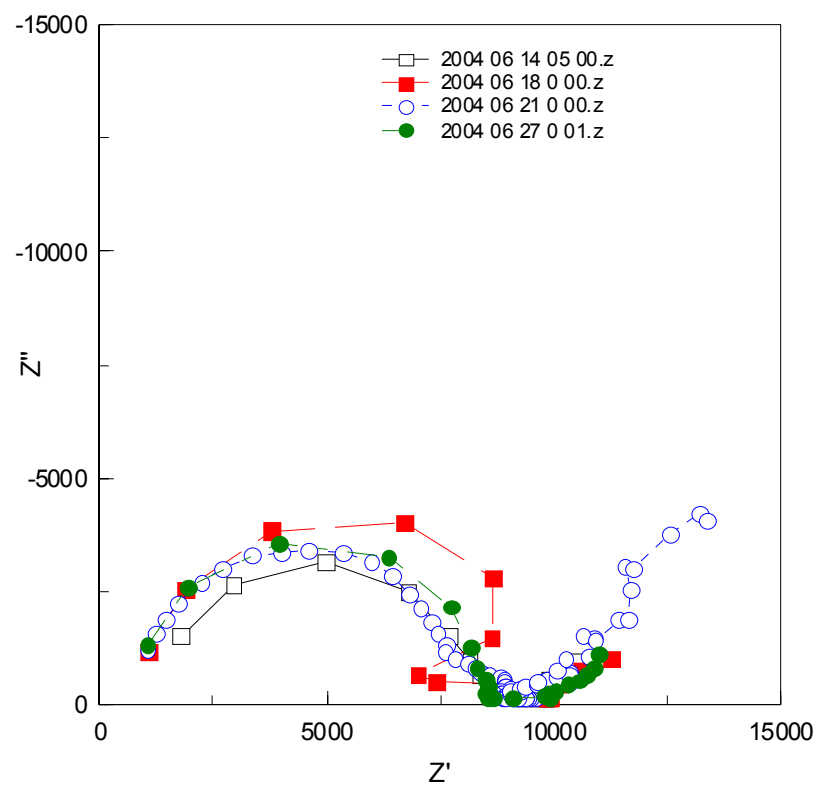

(b)

Figure 83. Evolution of TLEC spectrum for oxide film growing on alloy HT9 exposed to supercritical water with 5000 ppb of dissolved oxygen: (a) modulus and phase angle, (b) - Nyquist plot. 


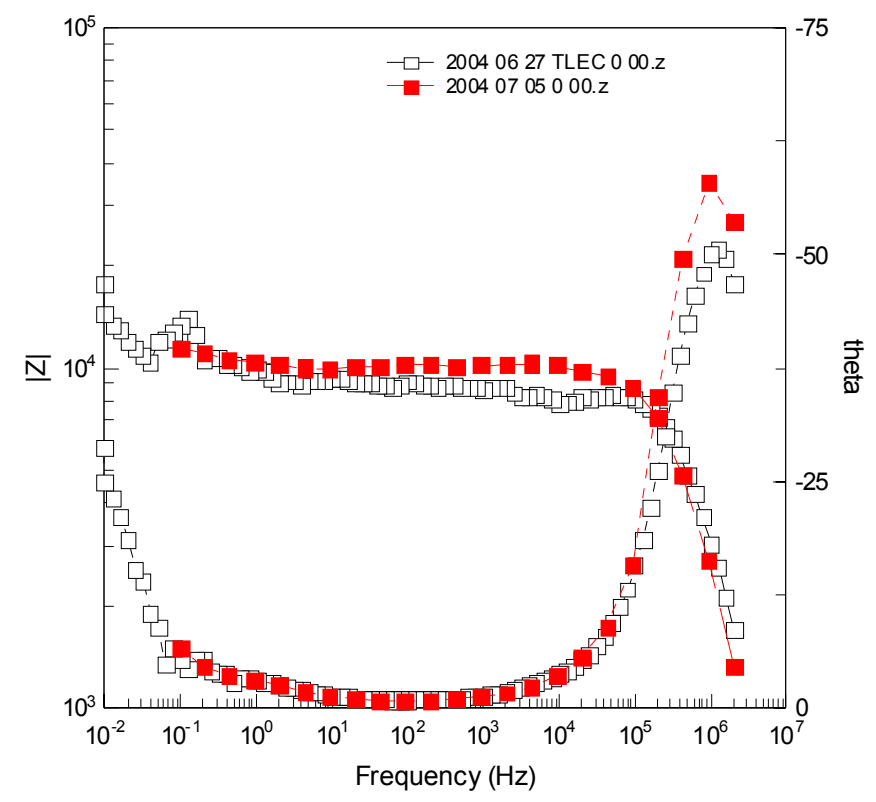

Figure 84. Evolution of TLEC spectrum for oxide film growing on alloy HT9 exposed to supercritical water with $50 \mathrm{ppb}$ of dissolved oxygen

\section{In situ Monitoring of Water Chemistry in Generation-IV SCWRs}

During the reporting period, we also estimated the potential use of the SRI-developed CDE technique for building in situ sensors for monitoring water chemistry in the heat transport circuit of Generation-IV SCWRs. Two types of sensors are envisioned: (1) a monitor to measure the oxide film stability of in-vessel structural materials, and (2) a water conductivity sensor.

The ability of the CDE arrangement to provide in situ continuous data on formation, growth, and transport properties of the oxide films is demonstrated by the data shown in Figure 49. Online processing of the impedance spectra measured with CDE specimens in contact with each other and with a controlled distance between them provides information on the rate of charge and mass transfer across the film. The sensor also provides data on the thickness of the oxide film as demonstrated in previous sections.

A special set of experiments has been performed to demonstrate a capability of the developed CDE probe to follow evolution of the film transport properties with time under variable water chemistry conditions simulating reactor startups, shutdowns, and implementation of the advanced water chemistry technologies to suppers radiolytically produced oxidizing 
species. An example of using CDE as in-situ monitor of stability of oxide film is shown in Figure 85. Alloy 690 samples have been exposed first to supercritical water with 500 ppb of dissolved oxygen. A clear trend of A690 to form oxide film at these conditions was observed. Such behavior of structural material indicates low susceptibility to SCC. After $\sim 400$ hours of exposure, dissolved oxygen level was increased to $2500 \mathrm{ppb}$ to simulate off normal water chemistry conditions (for example form unexpected shutdown of hydrogen additions). Within a matter of 100-200 hours, a detectable degradation of the oxide film was observed, indicating increased susceptibility of this structural material to SCC. In the field conditions this information may be easily collected and processed to determine the current status of structural materials in terms of their susceptibility to SCC and other forms of environmentally assisted degradation.

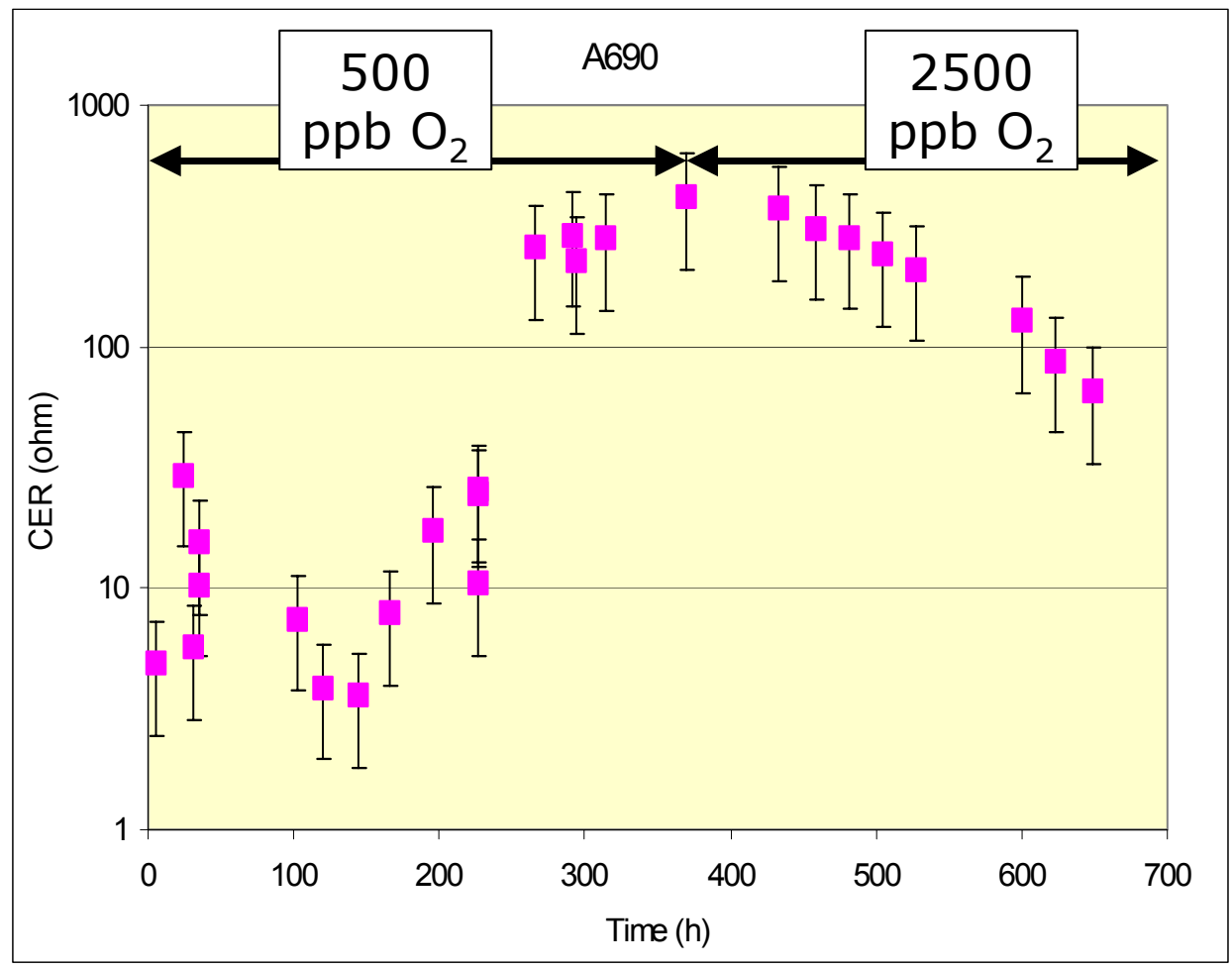

Figure 85. Film response to change in oxidizing conditions.

We also evaluated a potential use of the CDE arrangement as a robust sensor to monitor the conductivity of water in a wide range of temperatures, starting from room temperature up to the operating temperature of an SCWR. Because the distance between electrodes can be controlled and measured with high accuracy and because the electrical resistance of the water can be measured using a four-probe $\mathrm{DC}$ or $\mathrm{AC}$ technique, the resistivity of water can be 
estimated from these two measurements. The ability of the CDE arrangement to monitor water resistance in a few microns gap between the CDE specimens (electrodes) is demonstrated in Figure 86. Clearly, a suitable material—one that does not form a strong oxide film—should be selected as the electrode material. Ir can be considered as a candidate material, for example.

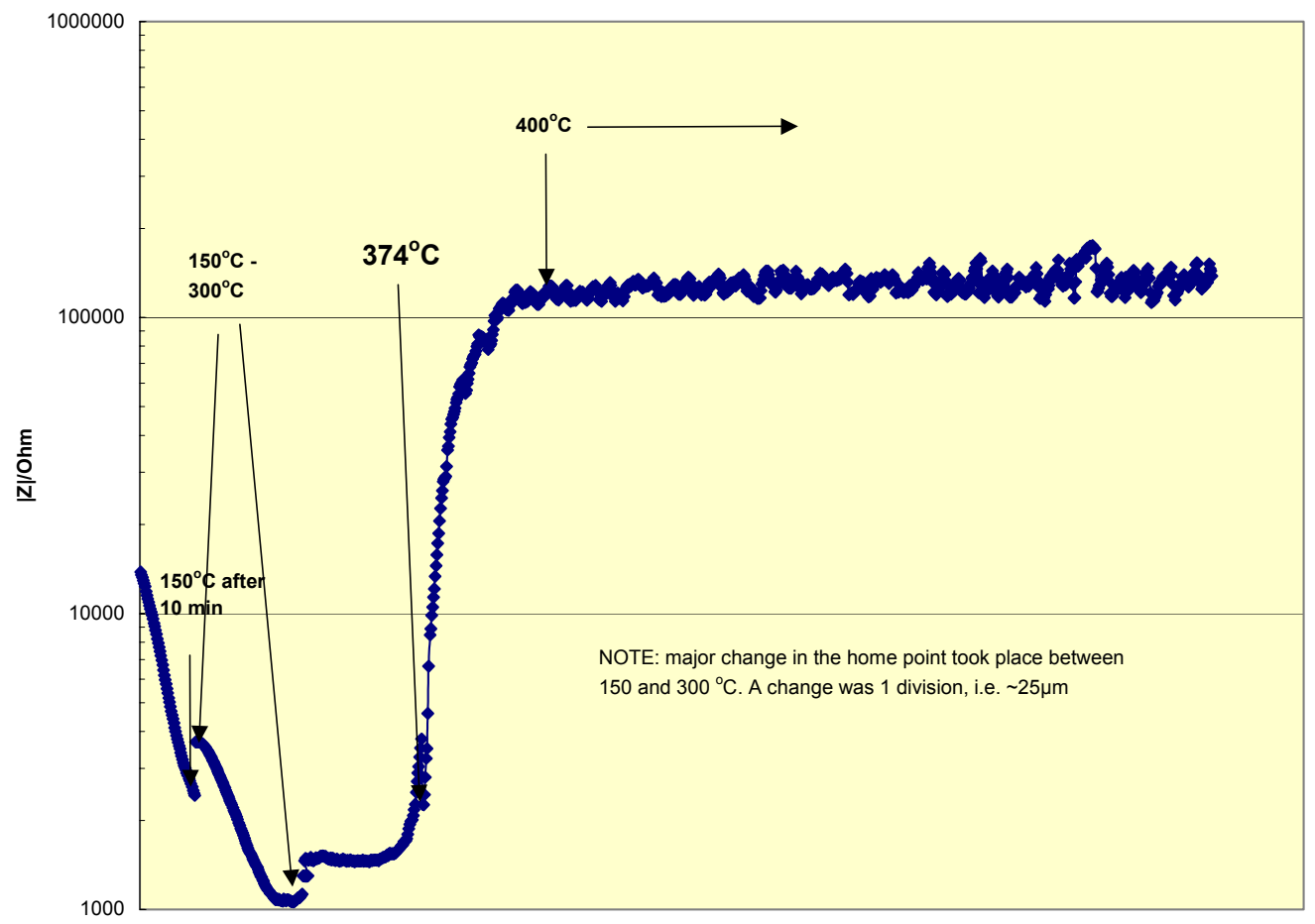

Figure 86. Monitoring resistance of the water between CDE electrodes during heating from room temperature to supercritical temperature.

\section{Chemical composition and thickness of the oxide films}

In situ study of the oxide films using the CDE technique should be complemented by a postexamination of the films to provide information on film morphology, composition, and thickness. These data are needed for calculating kinetic parameters and for refining theoretical models that describe film formation and growth. The morphology and thickness of the films were examined with SEM. The chemical composition of the films was obtained using the EDX technique and included here for 316 stainless steel as an example.

Figure 87 show low-magnification images of the working surface of CDE specimens with a diameter $4.3 \mathrm{~mm}$. The arrows in Figure 87 show two spots where film composition was 
examined with EDX. High-magnification images of the films reveal several distinctive features (see Figure 88). Arrows show three spots at the surface of the bottom specimen (see Figure 88a) and at the surface of the top specimen (see Figure $88 \mathrm{~b}$ ) where the film composition was examined.

Figure 89 summarizes the data on the film composition. These data are averaged and plotted in Figure 90 as a surface average composition, with the error bars corresponding to a standard deviation in the composition of a particular element over all EDX measurements. Preliminary analysis of the film composition indicated that the films consisted mostly of $\mathrm{Fe}$ oxides with $\mathrm{Cr}$ and $\mathrm{Ni}$ oxides as secondary components.

A cross section of the oxide film was also examined first with SEM (see Figure 91). Direct measurements of the film thickness $(\sim 10 \mu \mathrm{m}$ as seen in Figure 91$)$ were found to be in good agreement with in situ measurements using CDE. Such good agreement confirms the ability of the CDE technique to provide in situ information about film thickness, and accordingly about the kinetics of film formation and dissolution at supercritical temperatures.

The $\mathrm{Cr}$ content in the oxide film is of particular interest for the present work. The measured depth profile of the $\mathrm{Cr}$ in the oxide film (see Figure 92) indicates that the film is enriched with $\mathrm{Cr}$ (relative to the base metal). $\mathrm{Cr}$ also seems to be depleted at the outer surface of the oxide film. 


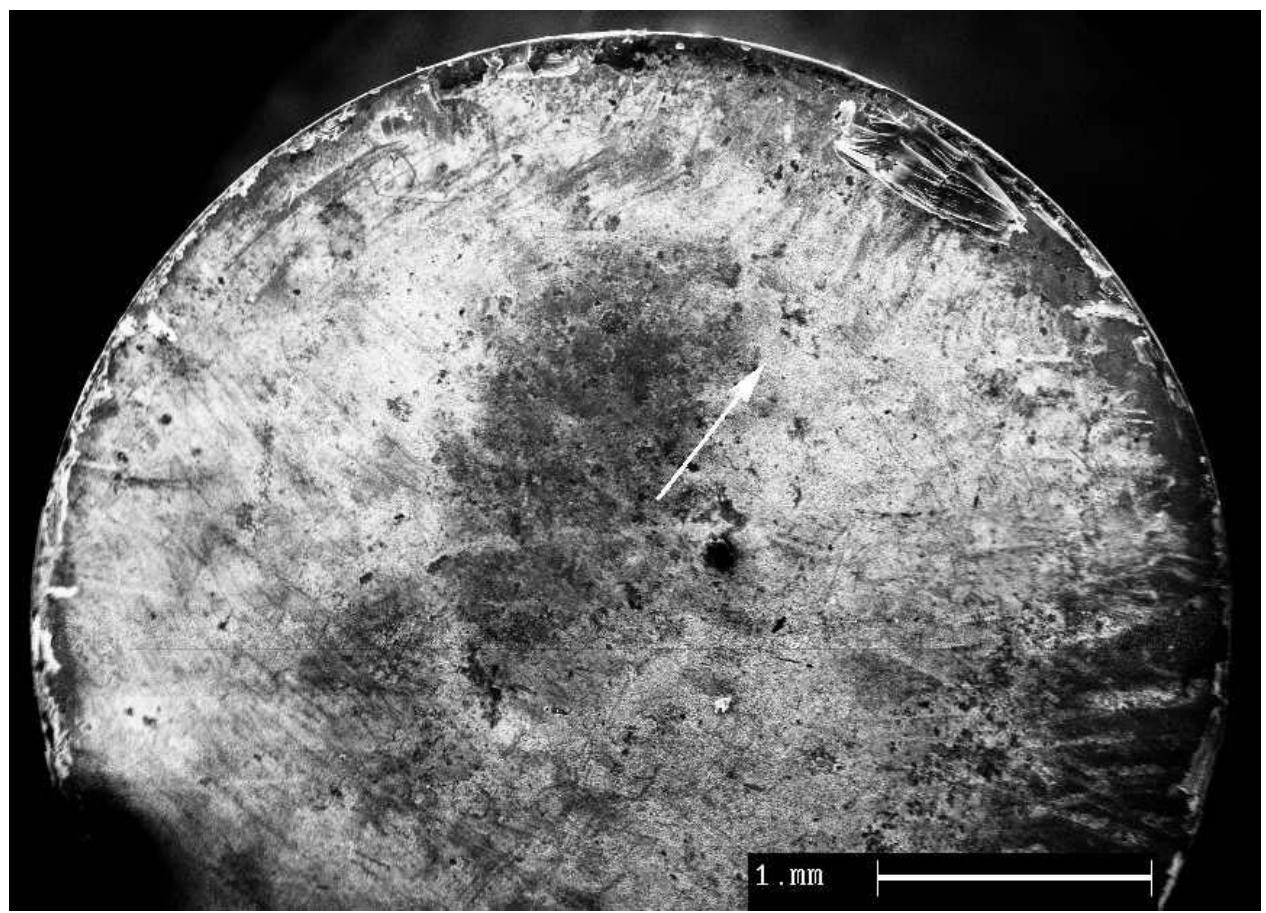

(a)

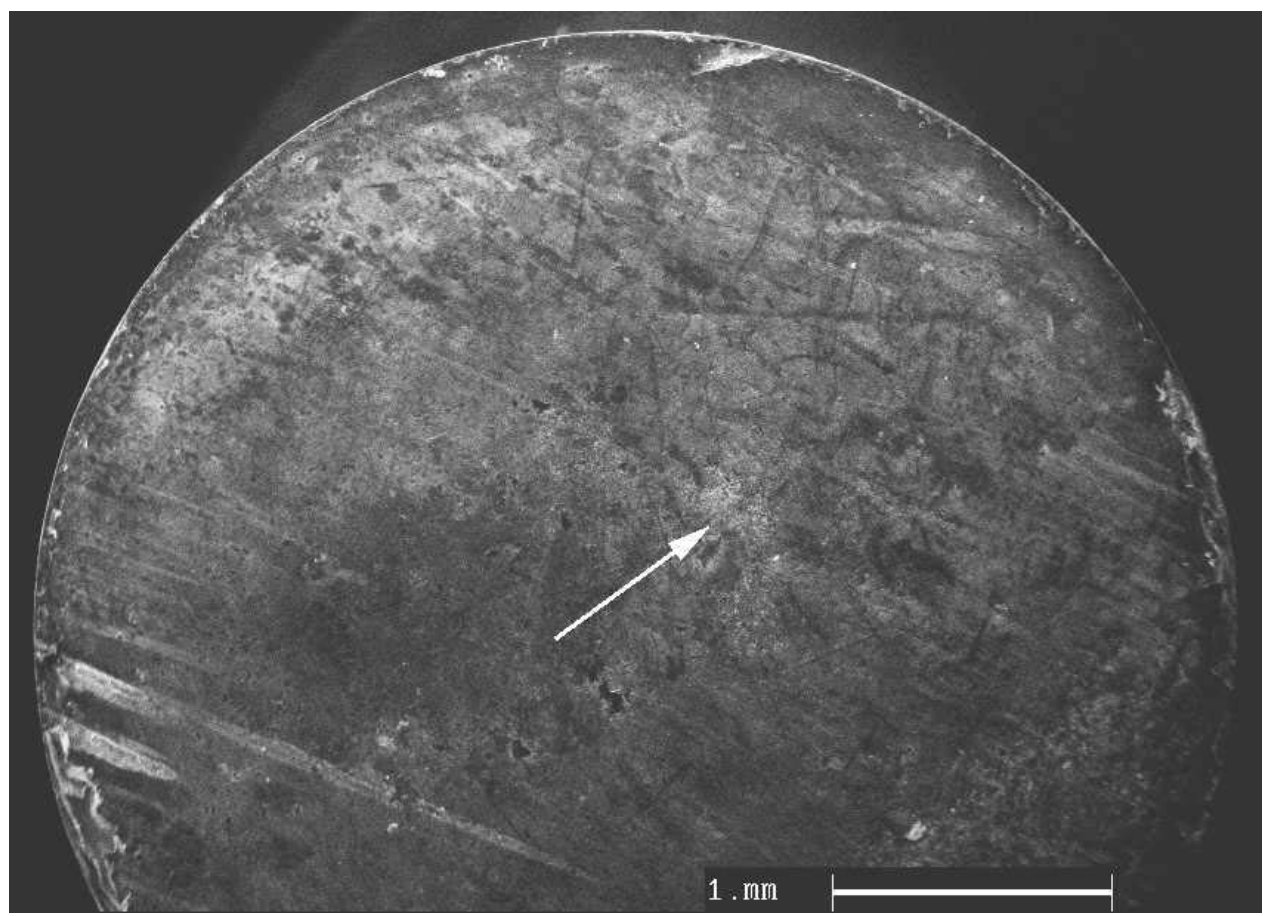

(b)

Figure 87. Low-magnification image of SS316 specimens: bottom specimen (a), top specimen (b). Arrows point to EDX analysis spots. 


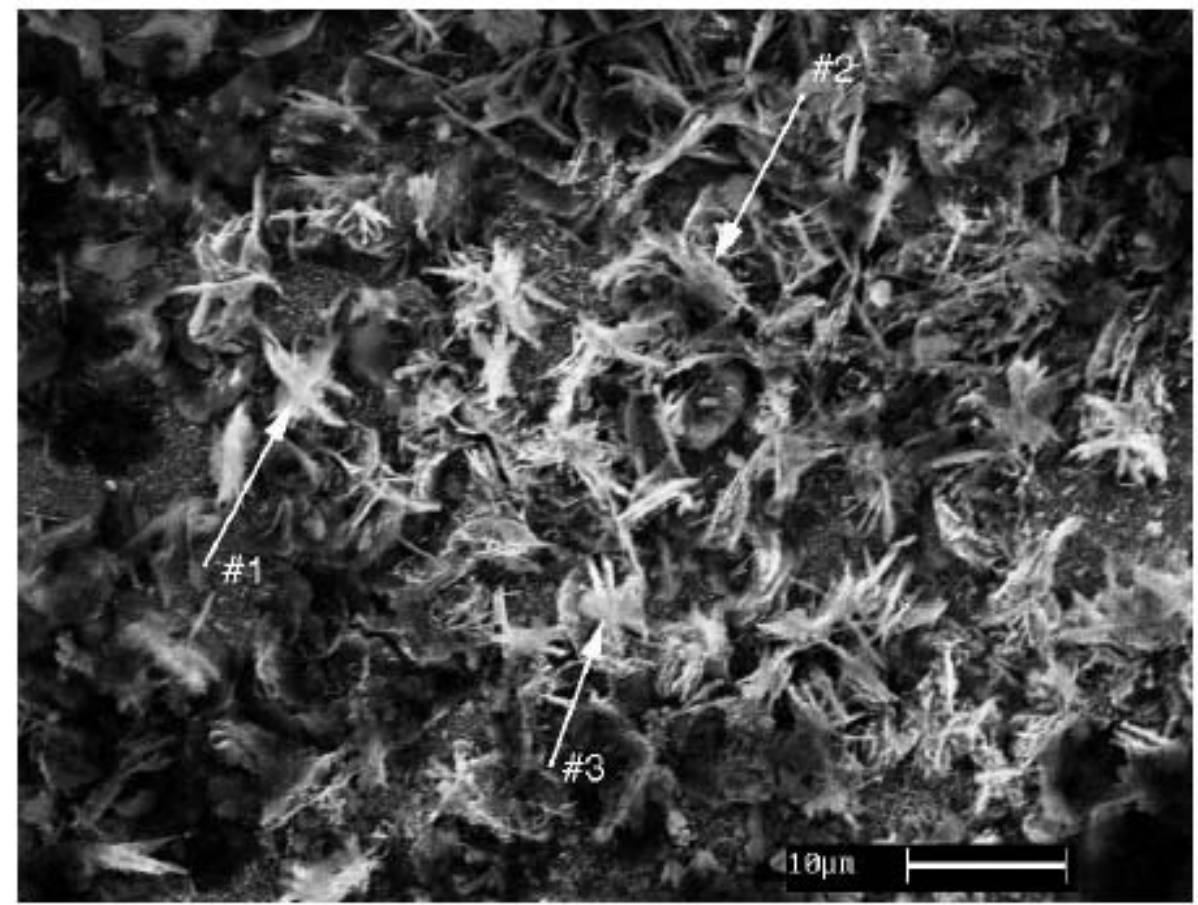

(a)

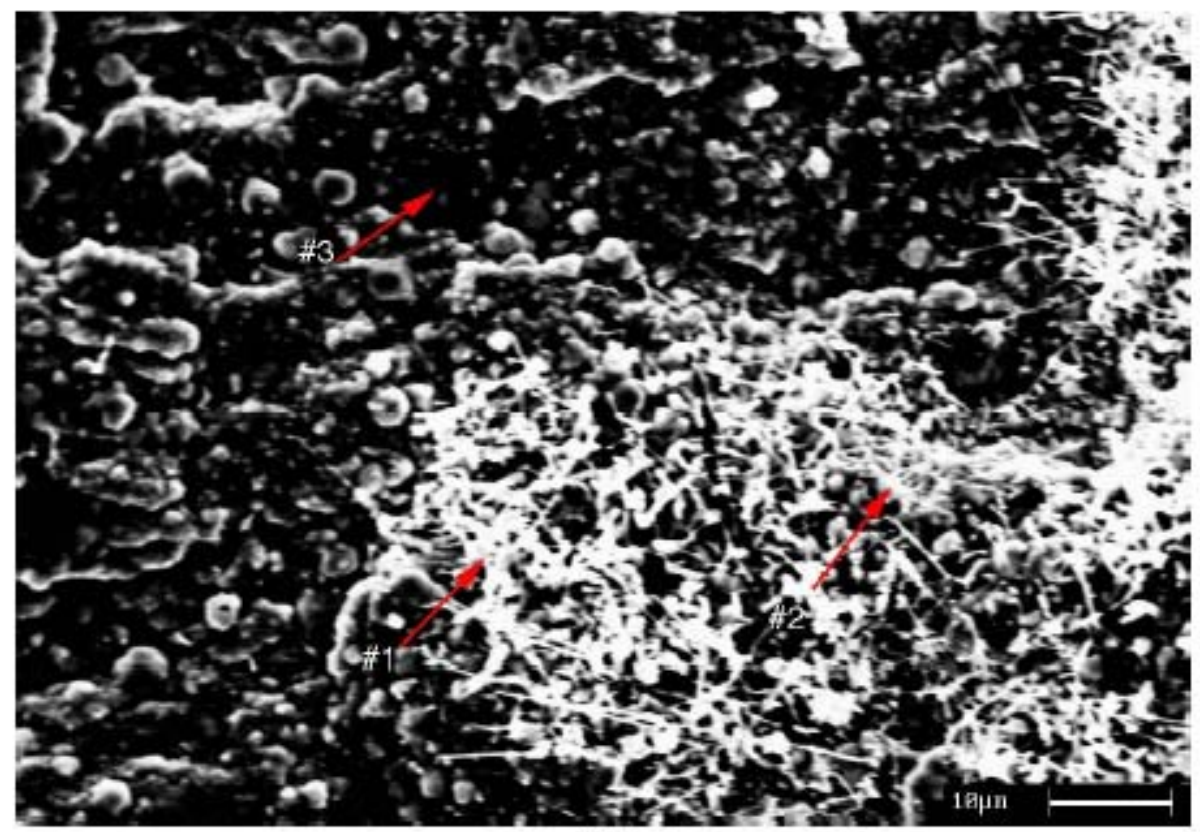

(b)

Figure 88. High-magnification image of SS316 specimens: bottom specimen (a), top specimen (b). Arrows point to EDX analysis spots. 


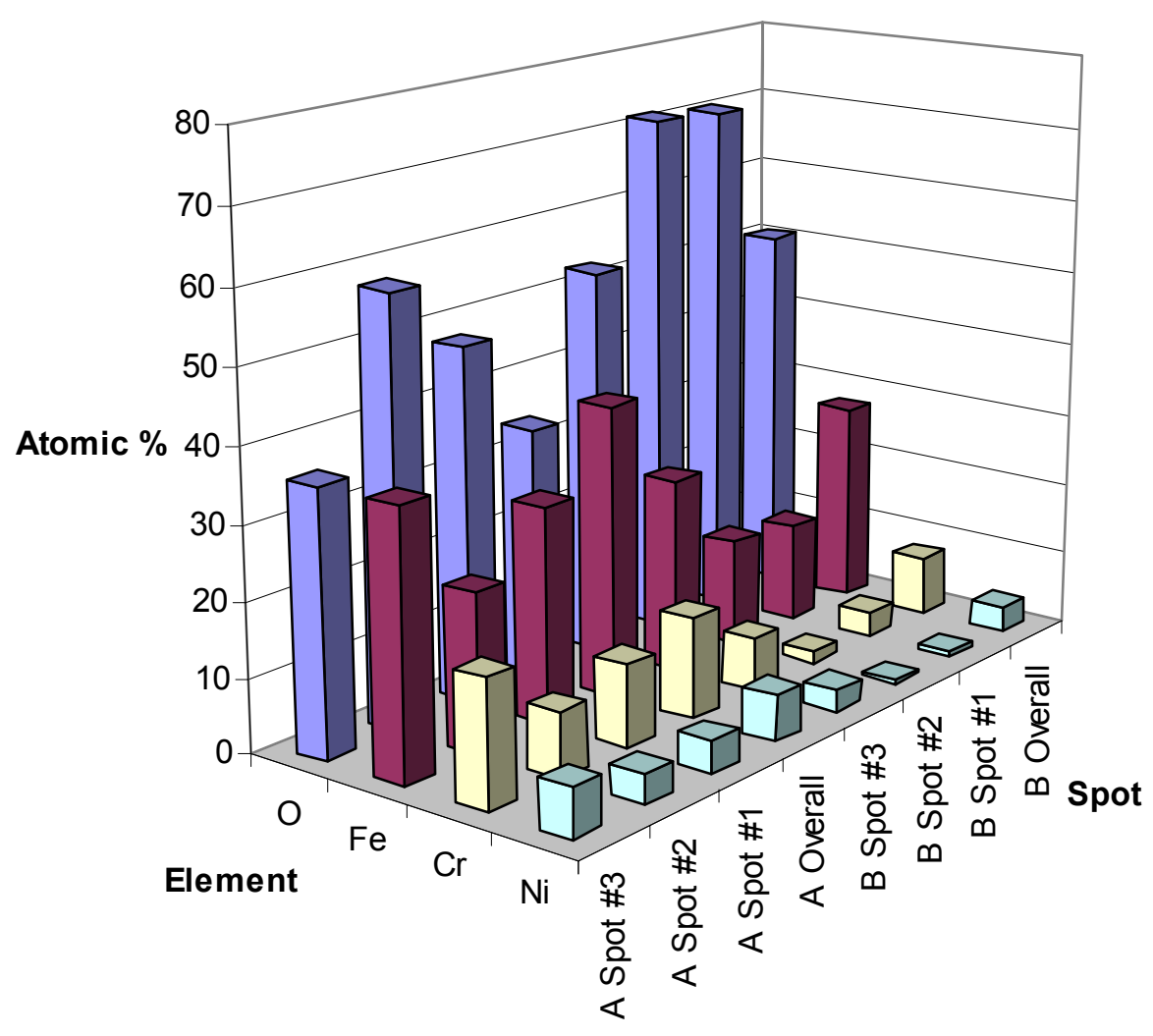

Figure 89. Composition of the oxide films on the SS316 specimen.

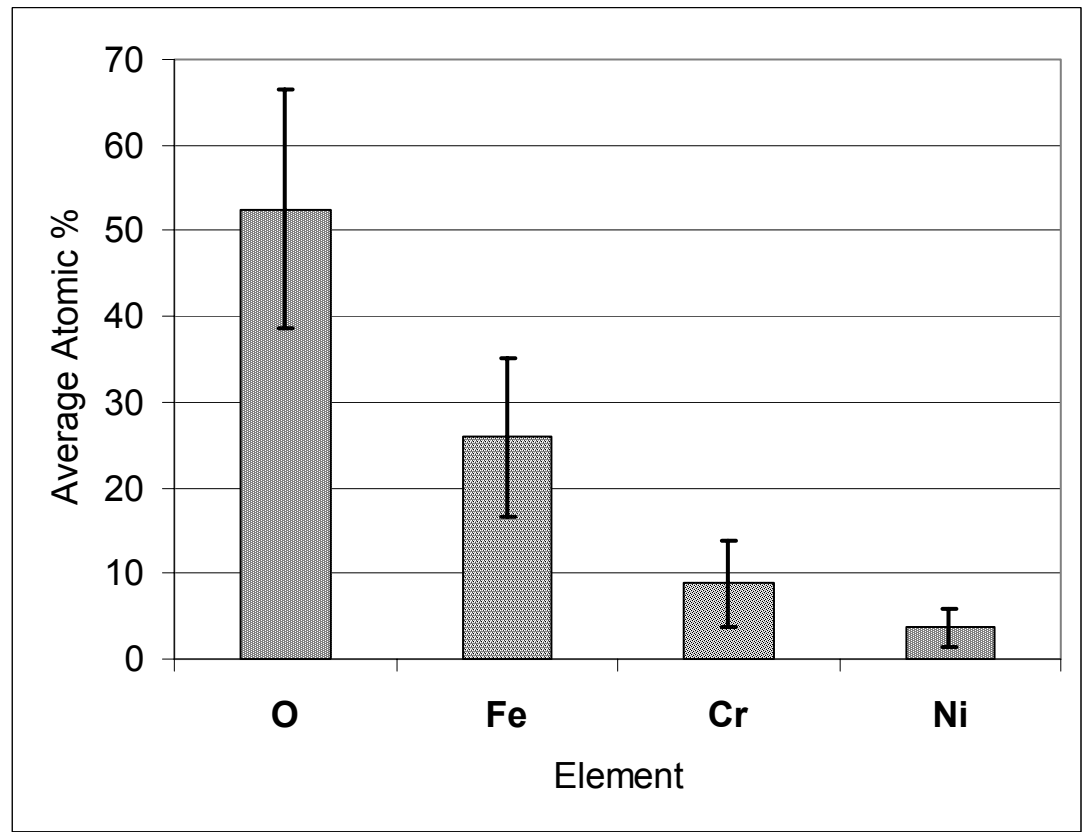

Figure 90. Surface averaged composition of the oxide film. 


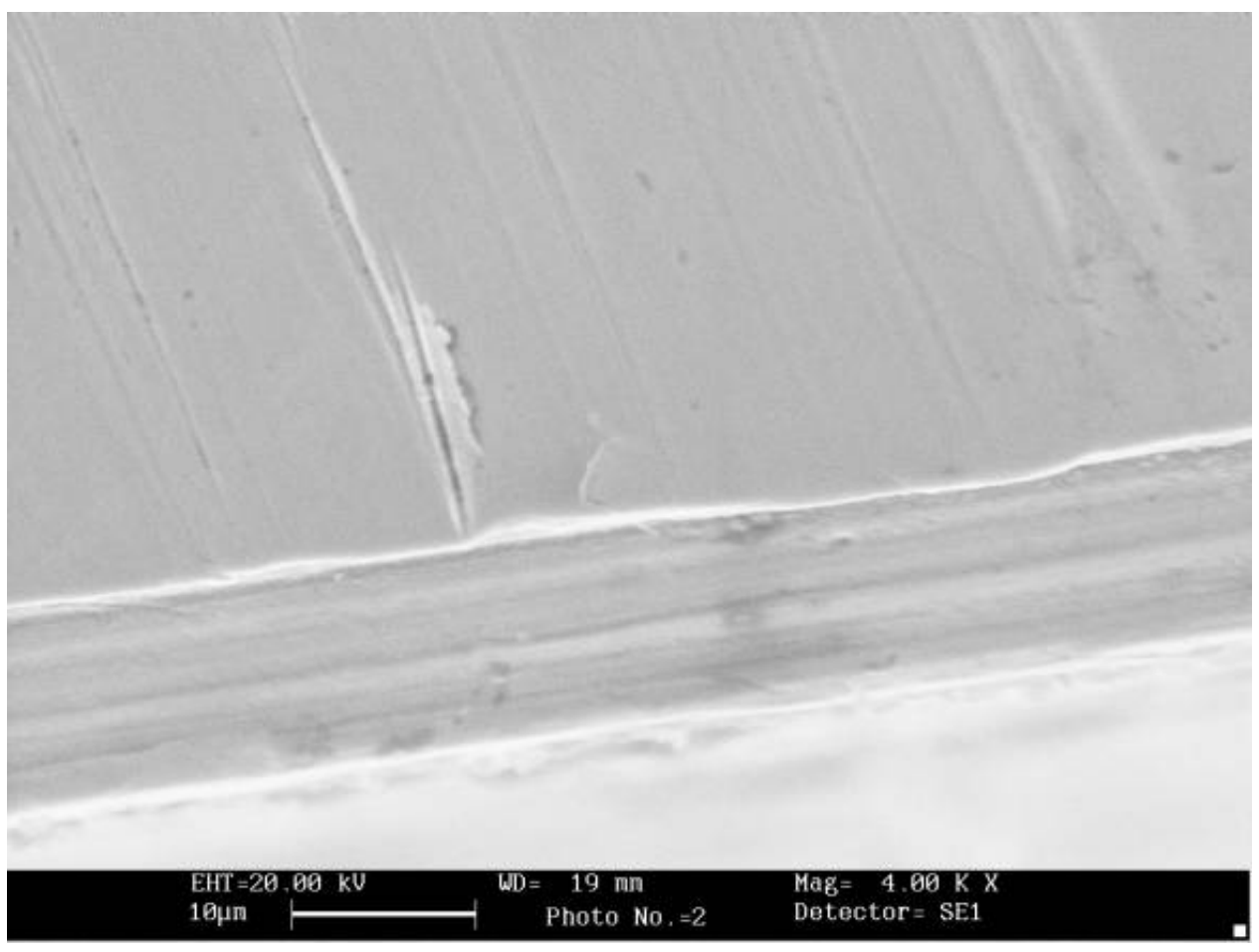

Figure 91. Thickness of the oxide film on the SS316 specimen.

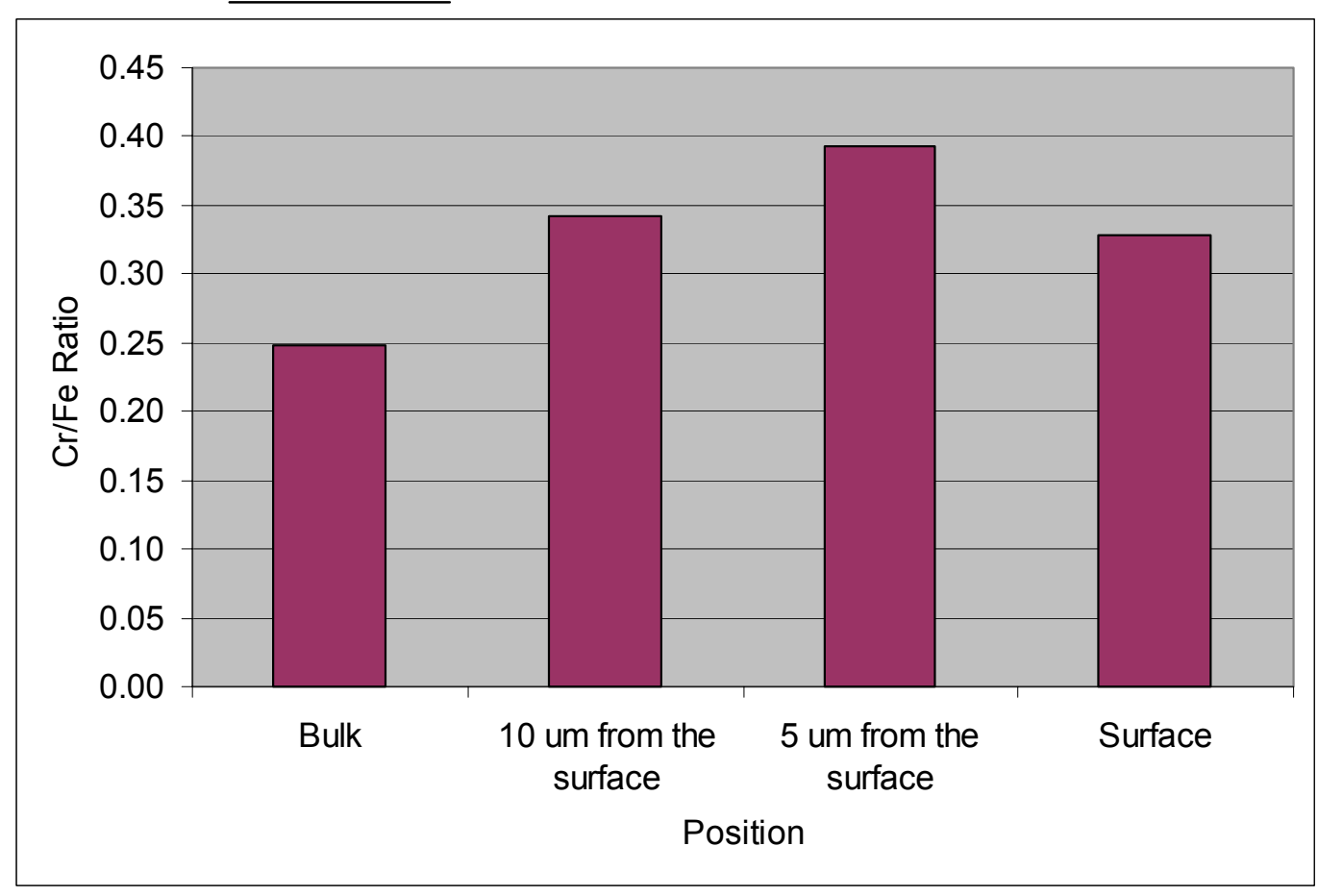

Figure 92. Cr profile in the oxide film formed on SS316 at $400^{\circ} \mathrm{C}$ and 3500 psi. 


\section{STUDY OF CRACK PROPAGATION IN INTERNALLY LOADED SPECIMENS}

To study crack growth in candidate structural materials SRI employed: FRASTA examination of the environmentally tested specimens; low-magnification analysis of the fracture surfaces; high-magnification analysis of the fracture surfaces; and analysis of crack front movement and estimation of crack growth rates.

SCC was observed for all tested stainless steels (304, 316, and 347). Fracture mode in all cases was not purely inter-granular. Examination of specimens made of nickel based alloys, alloy T91, and alloy HT9 revealed no discernible SCC. Accordingly, all further discussion of the fracture surface features and measured crack growth will cover stainless steels only.

\section{Post Examination of the Fracture Surfaces}

Typical exposure time of the SCC pre-loaded specimens was $\sim 1,000$ hours. Figure 93 shows the 304 stainless steel SCC specimen removed from the supercritical water reactor before the destructive examination. The formation of a surface film may be clearly seen from comparison with Figure 34 (before exposure).

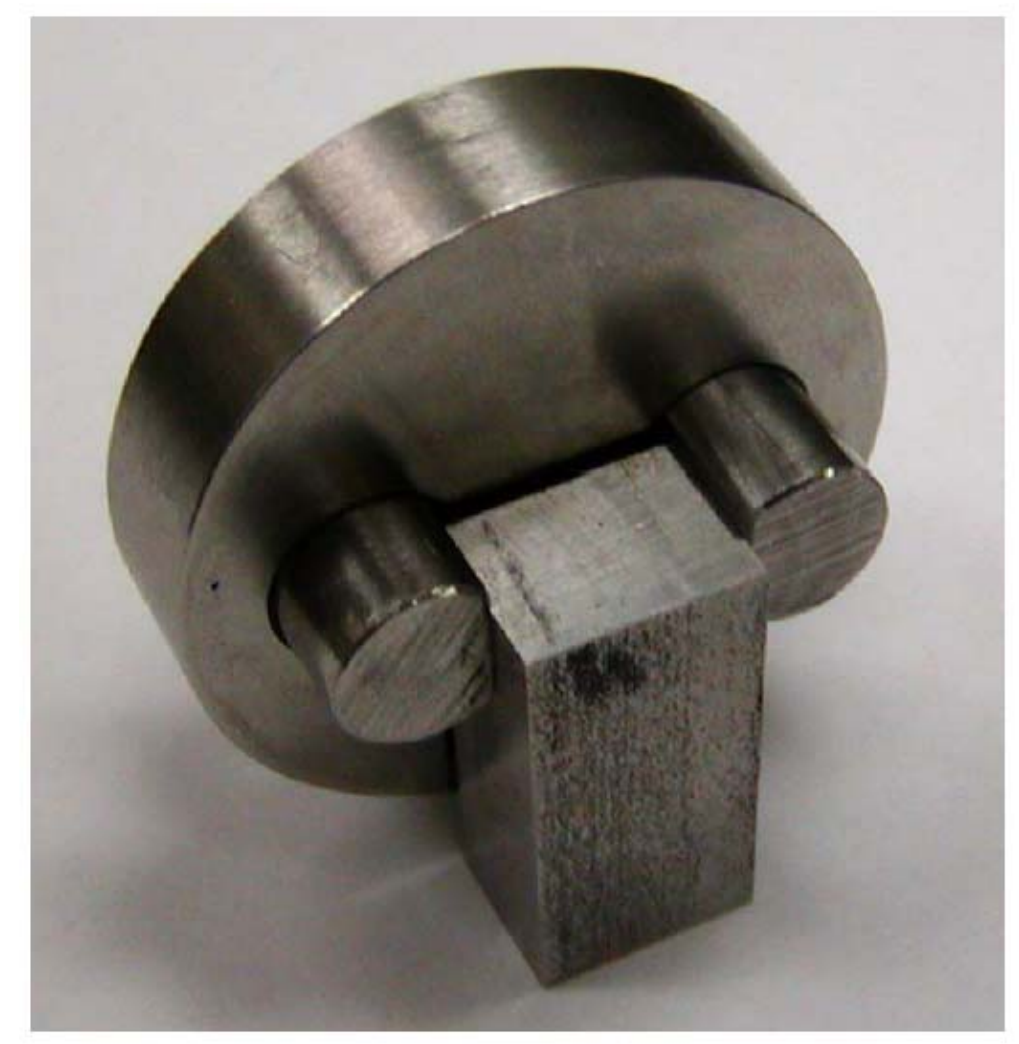

Figure 93. SSC specimen removed from the supercritical reactor. 
After the disc specimens were extracted from the autoclave, the loading wedges and pins were removed, and the discs were placed in an MTS loading machine that applied cyclic loading to extend the crack by fatigue mechanism. This marks the position of the crack front at the end of environmental test without deforming or damaging fracture surface. After extending the crack for a few millimeters by fatigue, monotonic loading was applied to force open the specimen to expose the fracture surfaces.

The FRASTA examination was applied first at lower magnification to cover the entire opened fracture surface. The objective of the low-magnification analysis was to determine the exact crack front location at the beginning of the environmental test. Doing so was necessary because it allowed us to observe the crack tip position on the specimen surface during the fatigue pre-cracking procedure; however, the internal crack front position differed from that of the surface because of the stress state difference and could not be determined before the environmental test.

The higher magnification FRASTA examination was then applied to the fracture surface area where the crack growth occurred during the environmental test. The higher magnification examination was applied twice to the same area: (1) as-opened fracture surfaces, and (2) after removal of oxides. During the long time exposure to the environment, oxide formation and build-up occurred in the crack opening, and this oxide deposits altered the fracture surface topography.

Before conducting the FRASTA examination, we obtained scanning electron microscope images of the fracture surface (Figure 94). The first encouraging observation is that visible "time marks" were produced on the fracture surface during restart/shutdown operations (Figure 95). This finding allows us to obtain information on a sequence of the fracture events (development of crack initiation sites and movement of the crack front) linked to the exposure times. Eventually, using FRASTA and surface "time marks," we were able to estimate crack initiation times and crack growth rates.

This preliminary examination was followed by a more detailed FRASTA analysis. The 3-dimensional profiles of the conjugate fracture surfaces of specimens were obtained using a scanning laser microscope. As mentioned above, the crack propagation process produces different amounts of local inelastic deformation in the material as a function of distance from the microfracture nucleation site. The difference in the local inelastic deformation results in a surface irregularity, i.e., an elevation profile on the fracture surface, thus providing a record of the history of microfracture events, including crack initiation. 


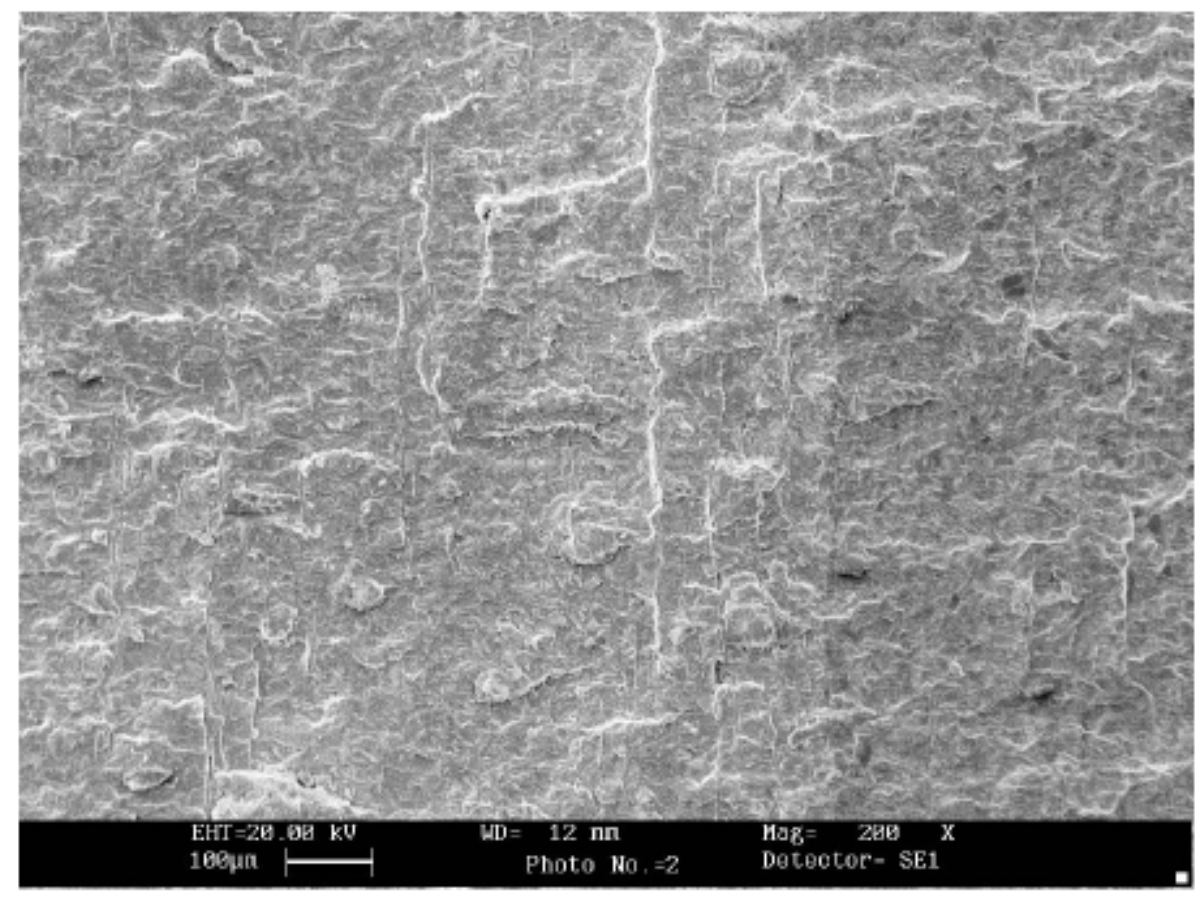

(a)

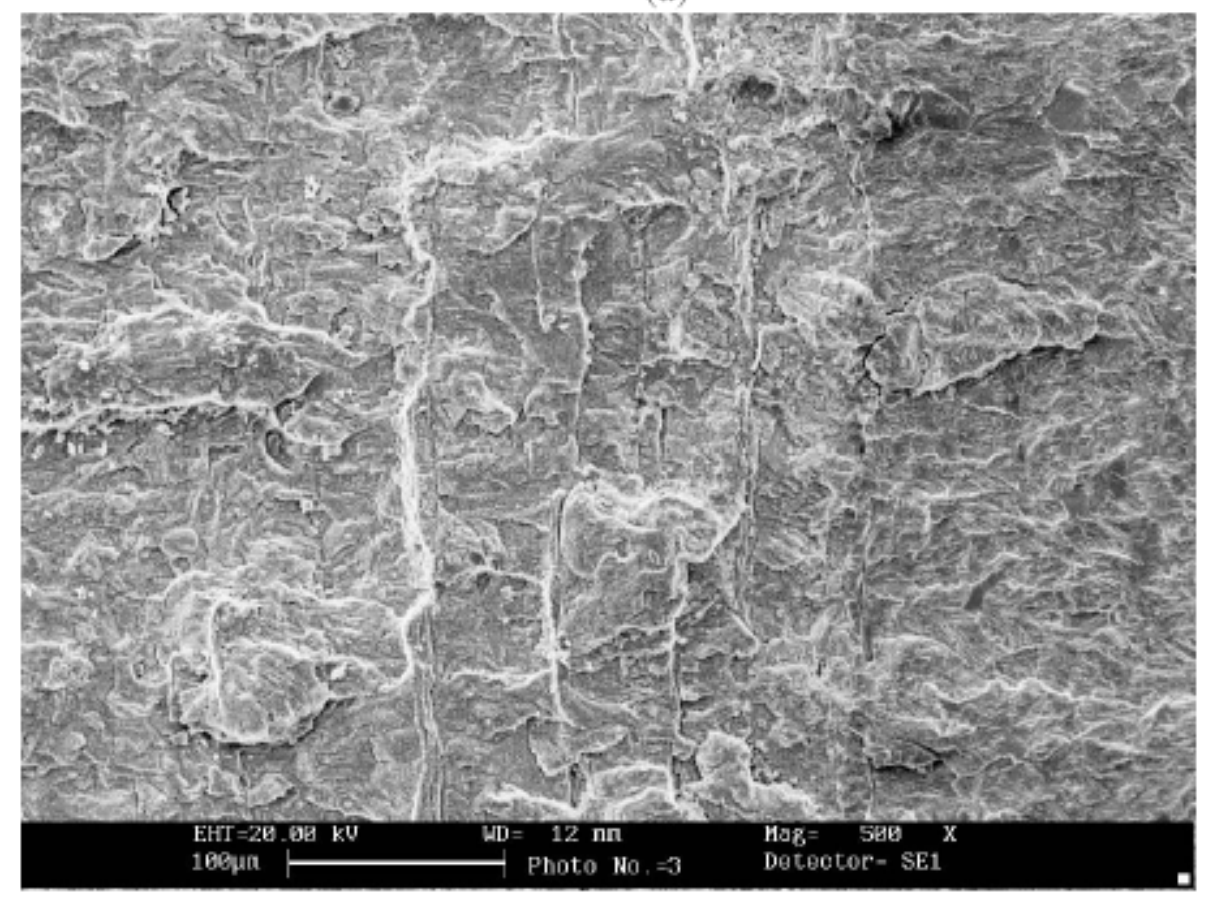

(b)

Fracture growth direction

Figure 94. SEM images of the fracture surface. 


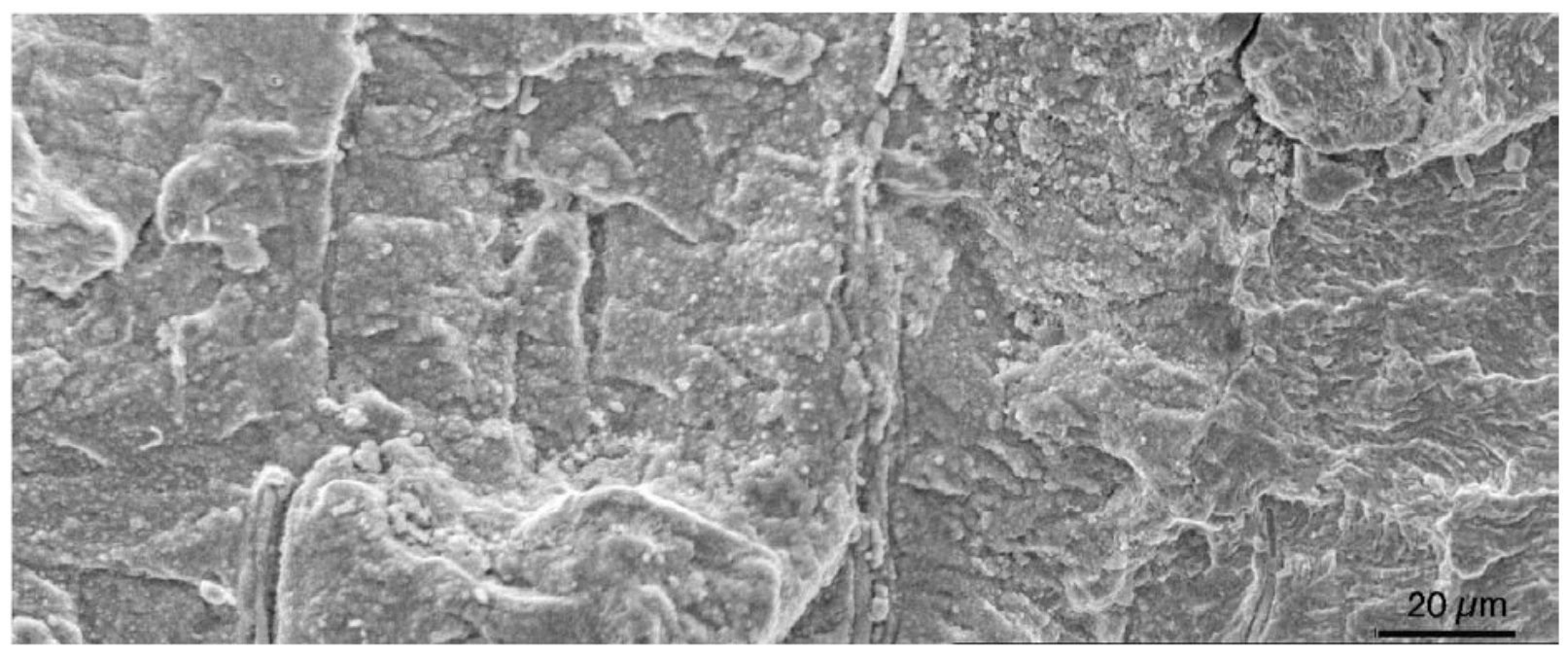

Figure 95. High-magnification SEM image of the fracture surface showing "time marks."

Crack behavior in the specimens tested in the environment is not easy to interpret, and it is frequently difficult to establish correlation with loading conditions. Commonly used method of determining the crack length such as DC potential drop measurement is very useful in providing continuous reading of crack tip position during the test. However, such a method requires careful calibration when applied to a new geometry, and also gives an averaged value through the thickness and also through the fracture process zone.

We were able to obtain more detailed information related to crack behavior by examining the conjugate fracture surfaces. The FRASTA technique characterizes the topography of conjugate fracture surfaces, juxtaposes conjugate fracture surface profiles on the computer, and assesses the inelastic deformation that occurs at the crack tip during crack extension by manipulating the spacing between the conjugate surfaces. Using the inelastic deformation on the fracture surface, we can reconstruct the crack growth history and microfracture processes and present the results in a graphic way.

Figure 96 shows the contrast and gray-scale topography images of the area covering the end of the fatigue pre-crack, crack growth during the test in the environment, and the beginning of forced opening after the test. In the gray-scale topography images white areas represent high elevation and dark areas low elevation. Images of Surface B were flipped horizontally for easier comparison with Surface A. The areas exposed to the environment were covered with oxide film and appear dark. Forced-open areas were highly reflective surfaces. In the dark area in the vicinity of a forced-open fracture surface, we observe several vertical markings. It is difficult to determine where the crack growth in the environment started through the visual examination of 
the SEM fracture surface images. Thus, we used FRASTA analysis to determine the significance of these markings and also the precise location of the transition from the fatigue precrack to the growth of the crack in the supercritical environment.

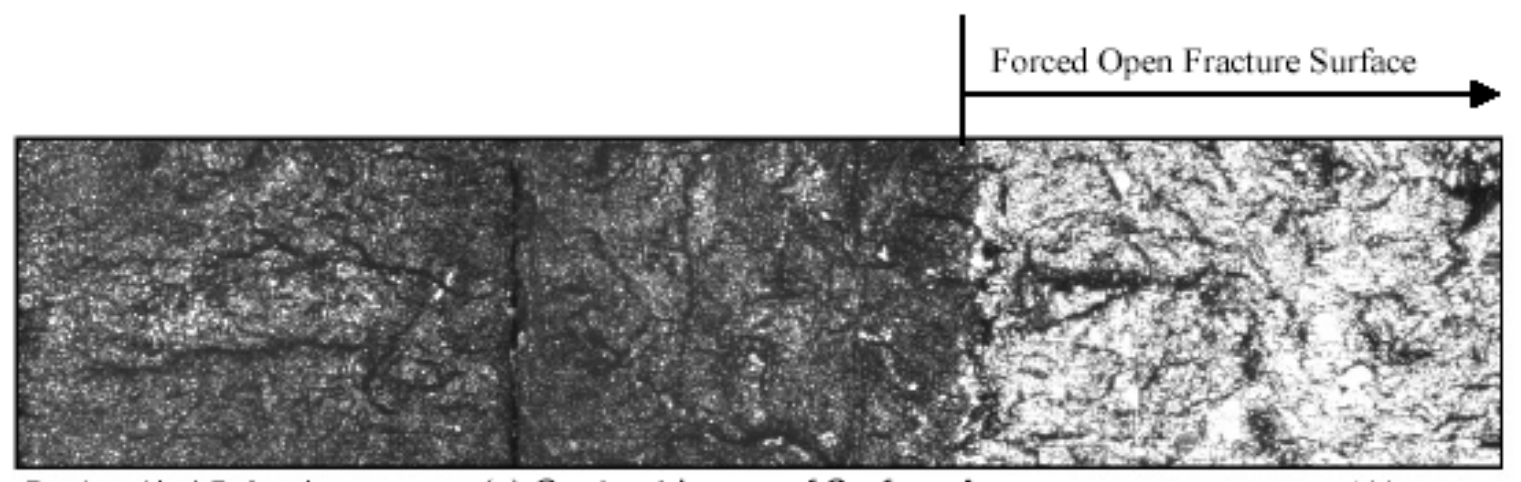

(a) Contrast Image of Surface A

$100 u m$

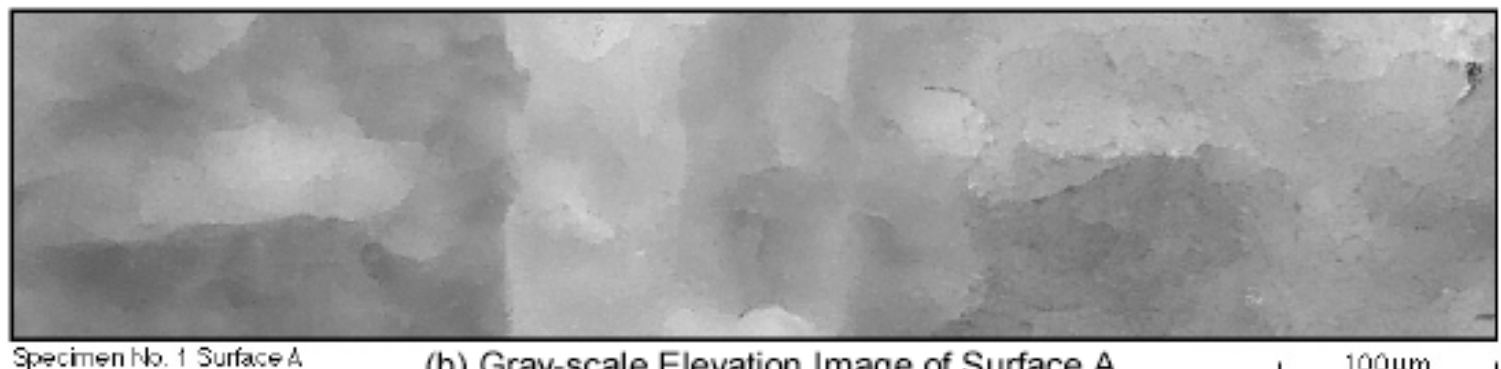

(b) Gray-scale Elevation Image of Surface A
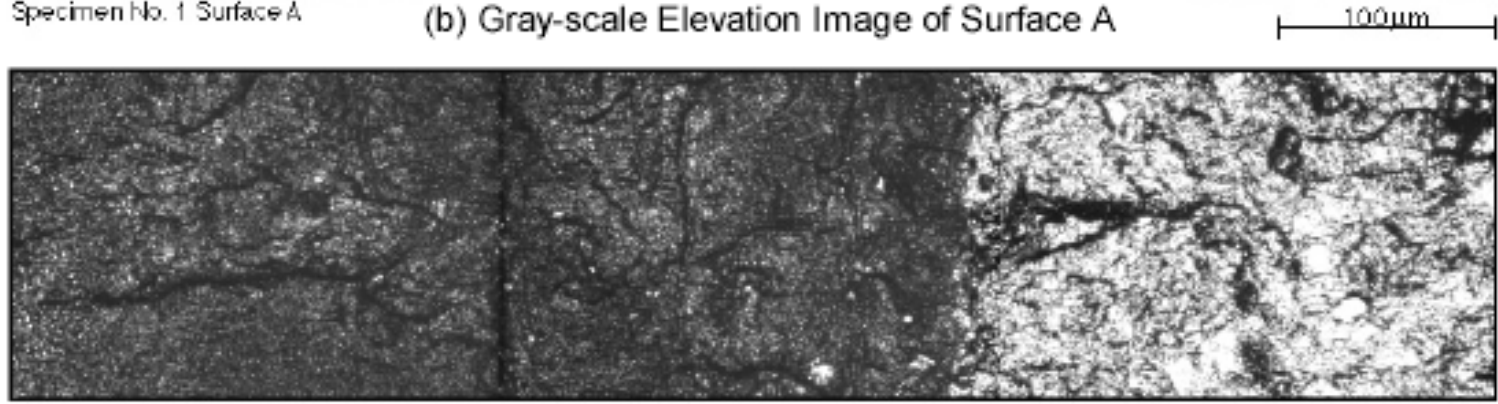

Specimen No. 1 Sufsce B

(c) Contrast Image of Surface B
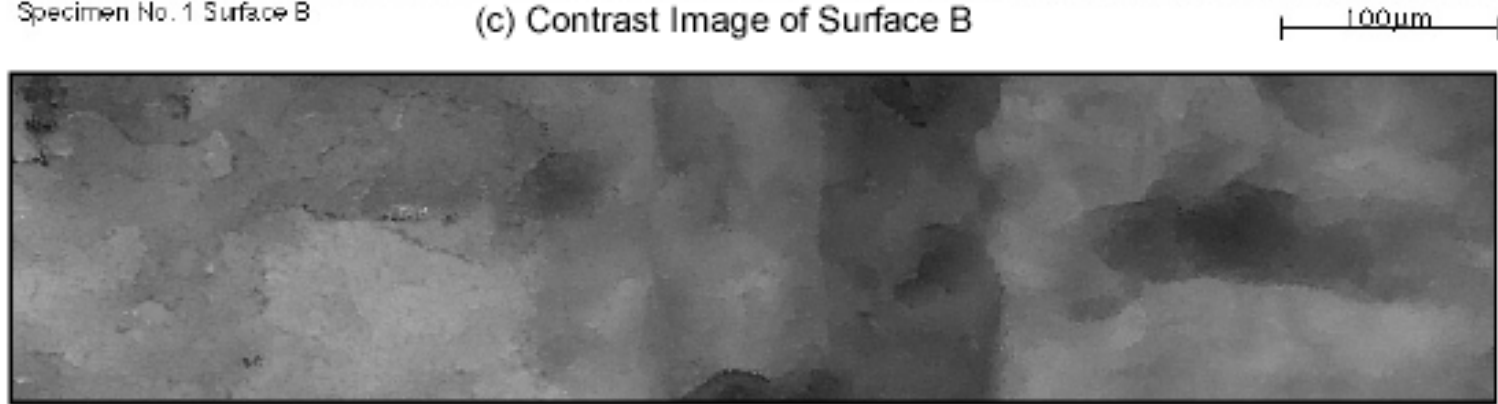

Specmenlk. 1 surace B

(d) Gray-scale Elevation Image of Surface B

$100 u m$

Figure 96. Contrast and gray-scale topography images of conjugate surfaces. 
Figure 97 shows a perspective view of the topography of the fracture surfaces. Several steps in elevation mentioned above are more clearly seen. These steps are parallel to the crack front.

Using the topography information, we reconstructed the fracture process. Some of the results are shown in the fractured area projection plots (FAPPs) of Figure 98. In the FAPPs we do not see a well-defined crack front. One of the reasons for this is that there are some errors in the elevation data; thus, when conjugate surfaces were matched, the crack front became a zone rather than a single line. The magnitude of elevation error in this analysis is not negligible because the surface reflection was relatively poor due to oxide film and the degree of deformation was small.

Figure 99 shows the contrast images of conjugate fracture surface areas where the crack growth occurred during the environmental test, together with the SEM image of the same area. The image of Surface B was horizontally flipped to make the comparison of features on the conjugate surfaces easy. The white frame in the SEM photograph (c) shows the area corresponding to the contrast images in (a) and (b).

It is interesting to observe the significant differences in the local gray levels (darkness) in the areas where the crack grew in the environment. By comparing the contrast images in (a) and (b) with the SEM image in (c), it is apparent that the dark areas in (a) and (b) appear to be whitish in (c). The higher magnification examination of the whitish areas in the SEM photograph revealed that these areas were covered by needle-like oxides and that the gray areas were covered by globular oxides.

The FRASTA results shown in Figure 100 reveal that the dark areas in Figure 99a and b, and the whitish areas in Figure 99c are the areas where the crack opening was filled (no gaps) during the environmental test. These results suggest that the spaces between the crack flanks at some local areas were completely filled with thick layers of needle-like oxides and that some gaps were left in other areas. Apparently, the growth of oxide layers was not uniform and depended on local conditions. This suggested that it was important to study the local material underneath the oxides. We thus removed the oxides by using electrochemical methods and applied FRASTA to the cleaned surfaces. 

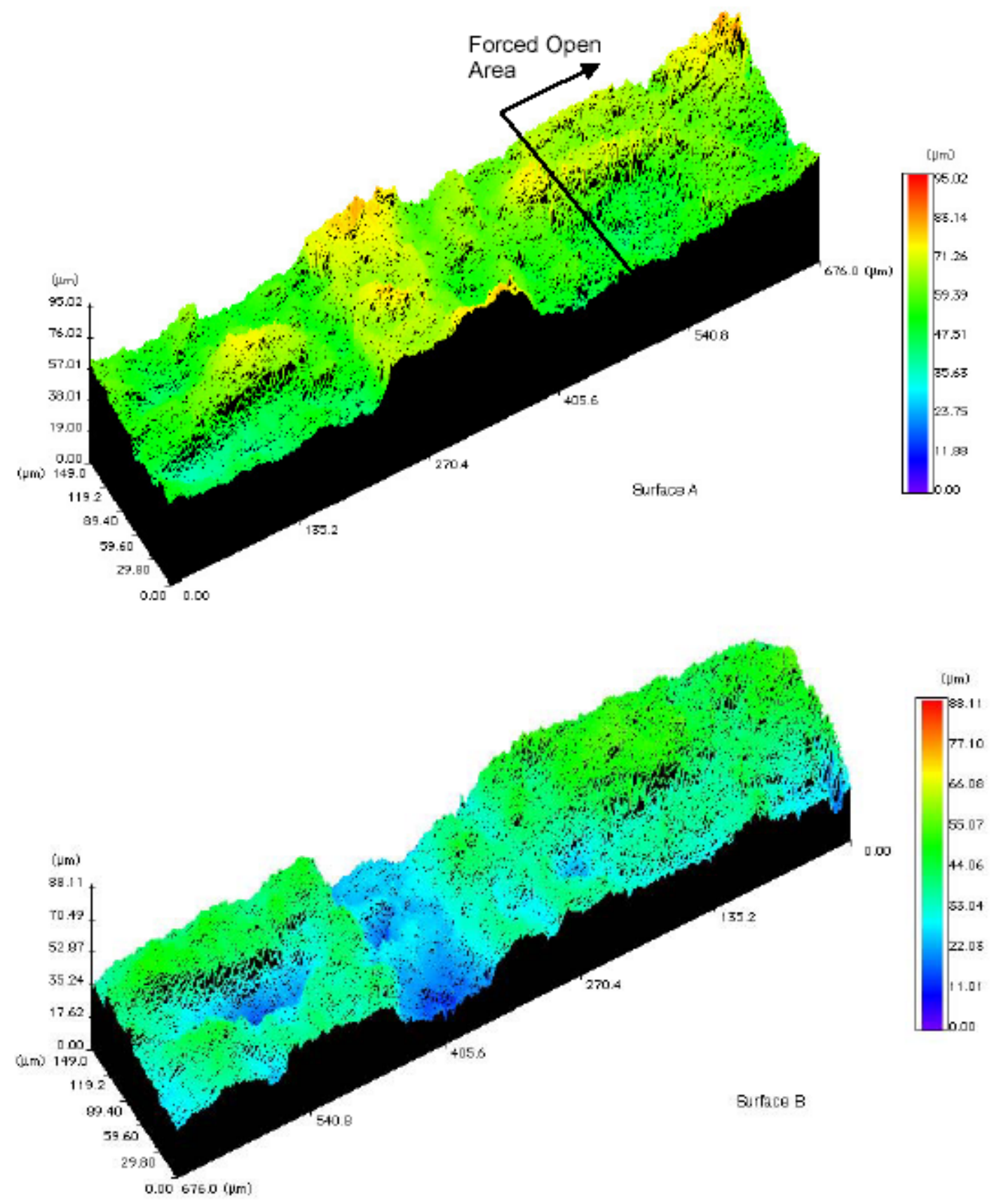

Figure 97. Perspective view of fracture surfaces. fracture surfaces. 

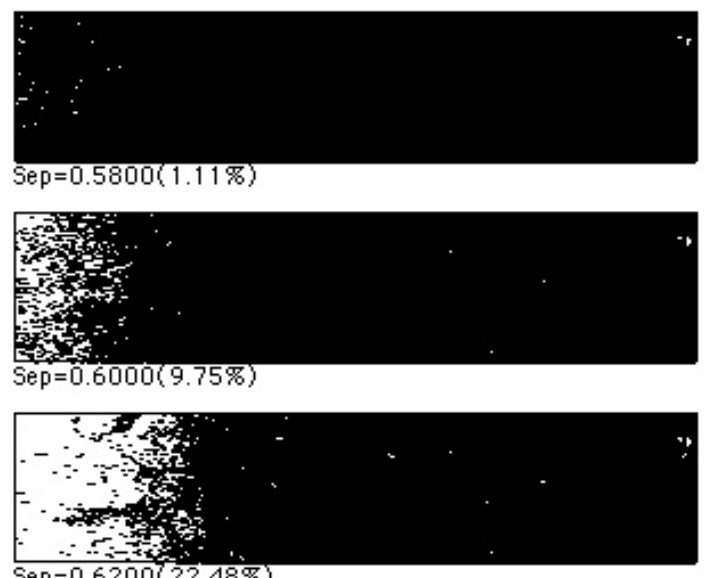

Sep $=0.6200(22.48 \%)$
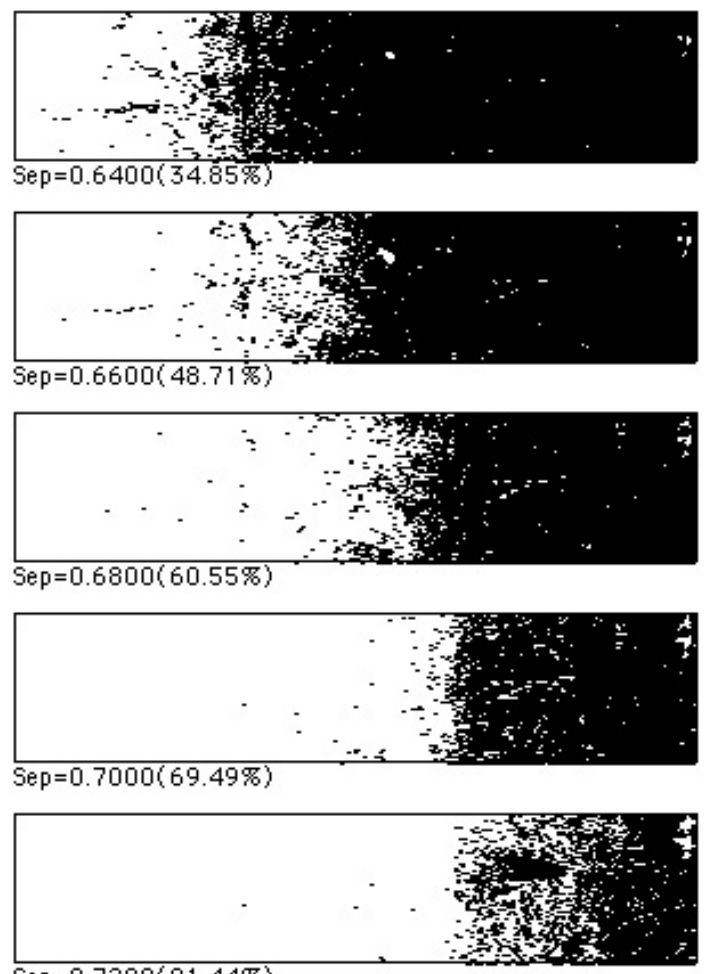

Sep $=0.7200(81.44 \%)$

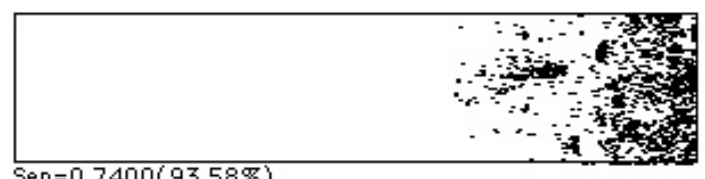

Sep $=0.7400(93.58 \%)$

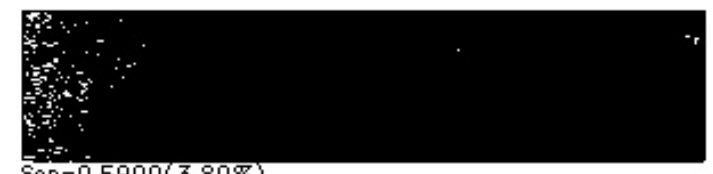

$\mathrm{Sep}=0.5900(3.80 \%)$
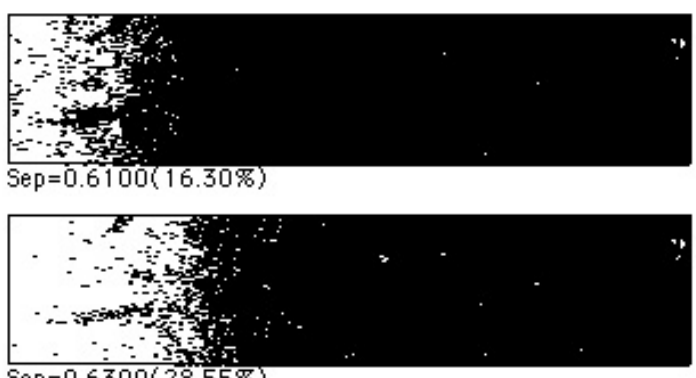

Sep $=0.6300(28.55 \%)$
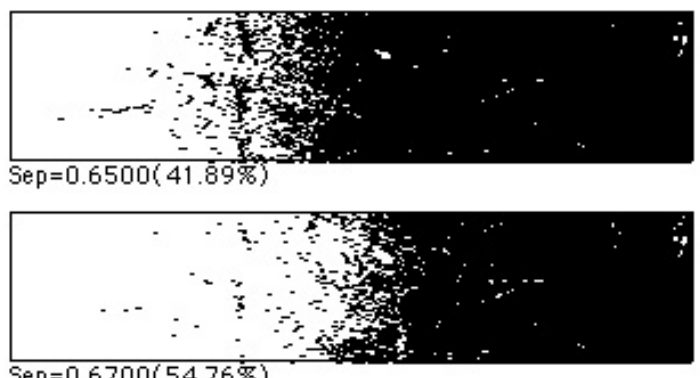

Sep $=0.6700(54.76 \%)$
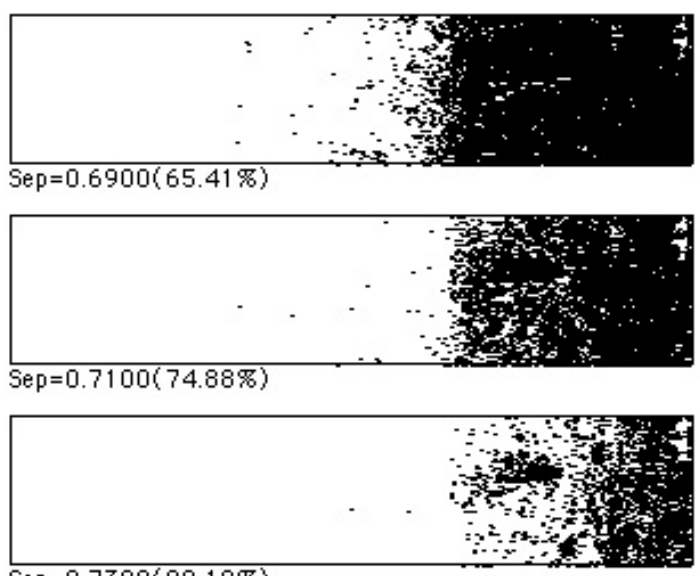

Sep $=0.7300(88.10 \%)$

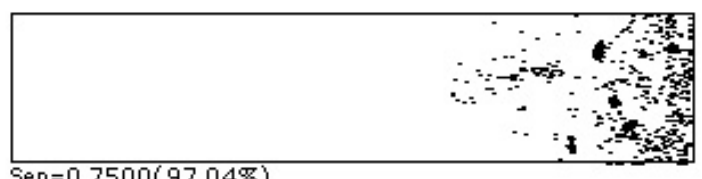

Sep $=0.7500(97.04 \%)$

Figure 98. A series of FAPPs showing the crack growth processes. The plots show hesitation in crack front movement at some locations. 


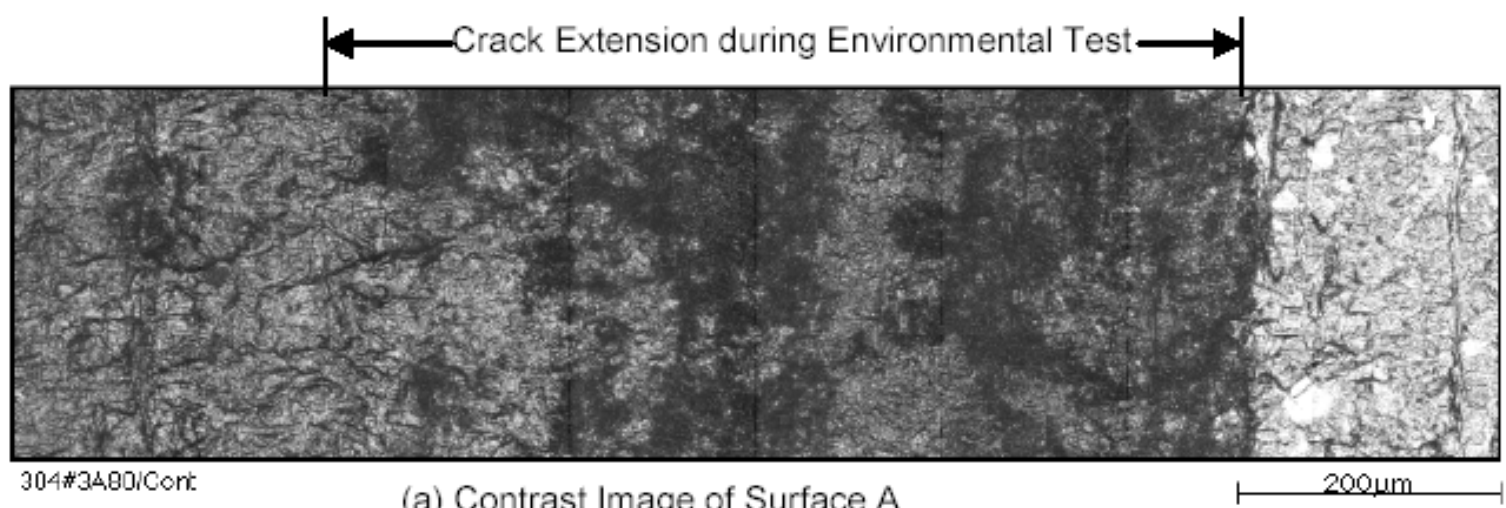

(a) Contrast Image of Surface A
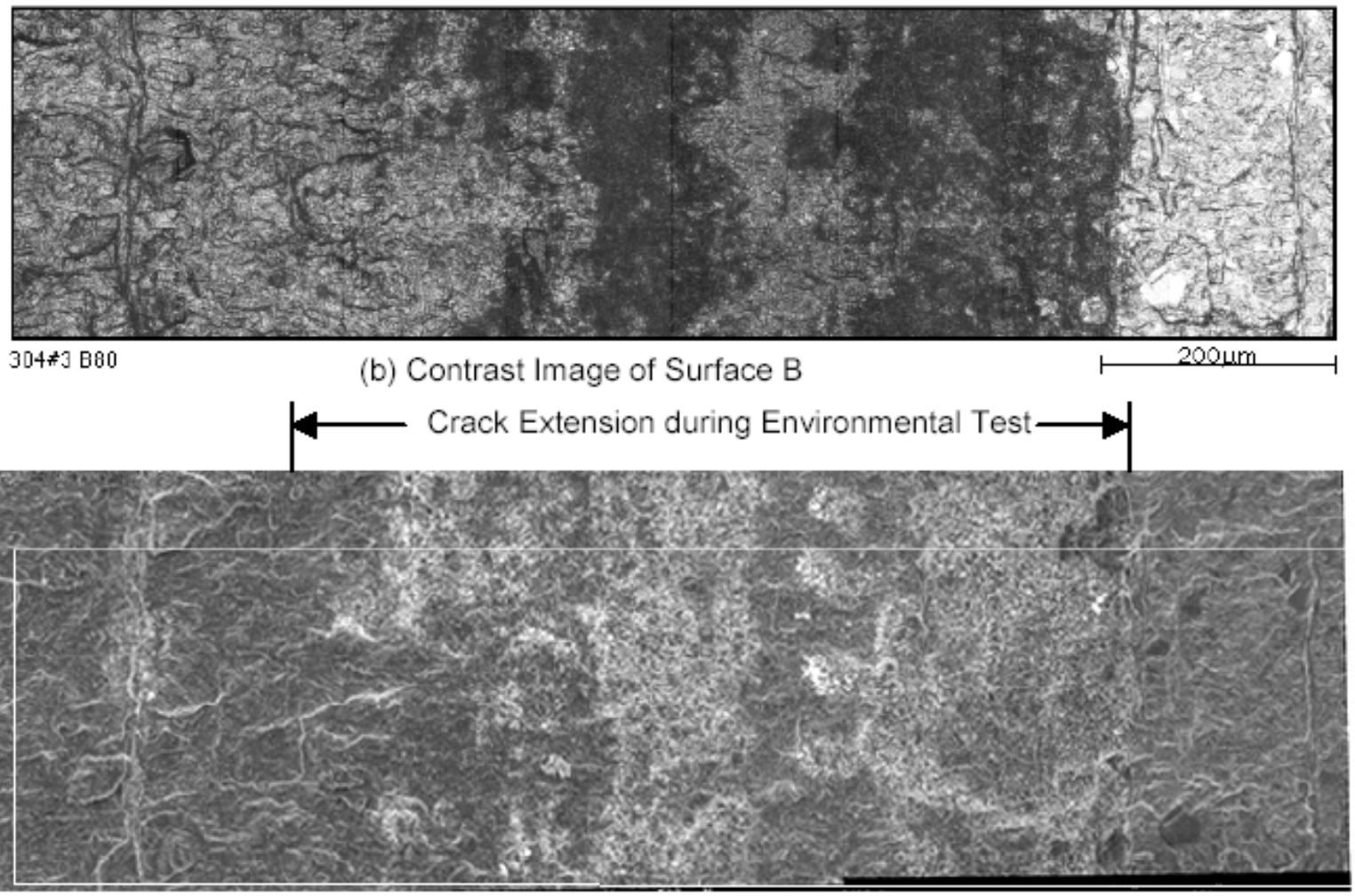

(c) SEM Photograph of Surface A

Figure 99. Contrast images of conjugate surfaces and a corresponding SEM photograph. (Surface B image was flipped to enable comparison of images.) 


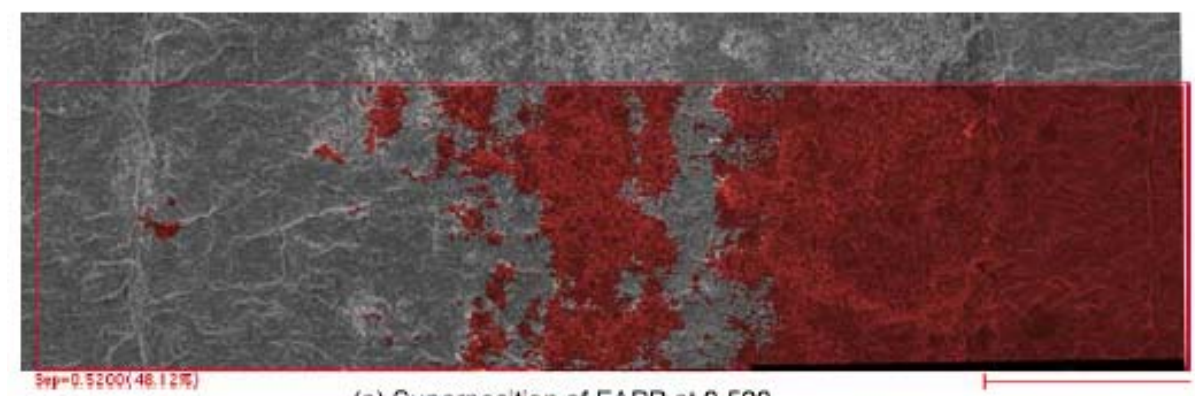

(a) Superposition of FAPP at 0.520

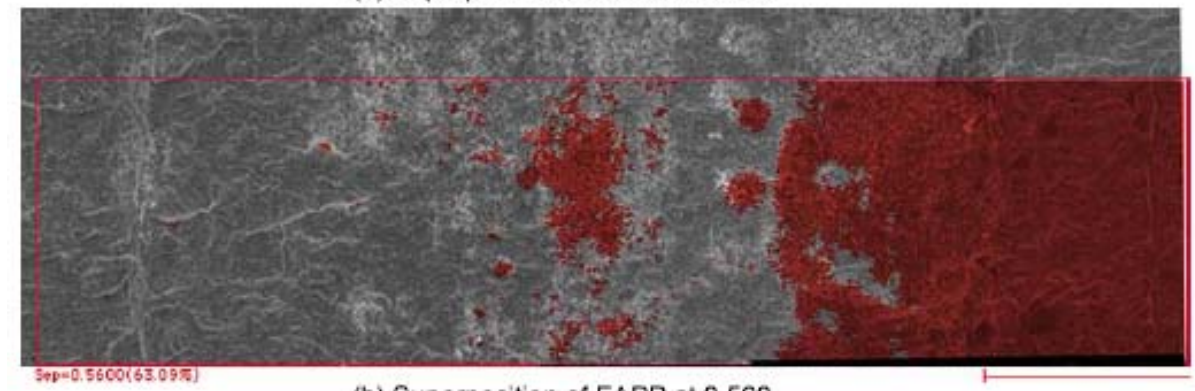

(b) Superposition of FAPP at 0.560

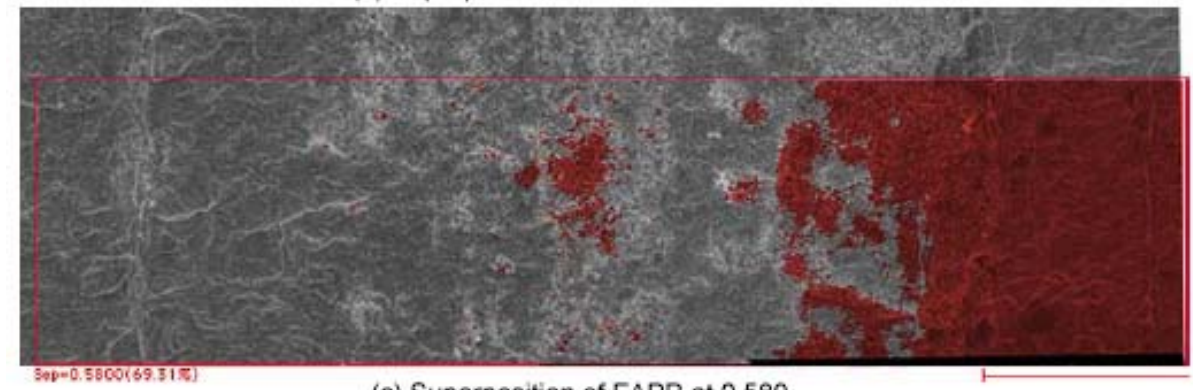

(c) Superposition of FAPP at 0.580

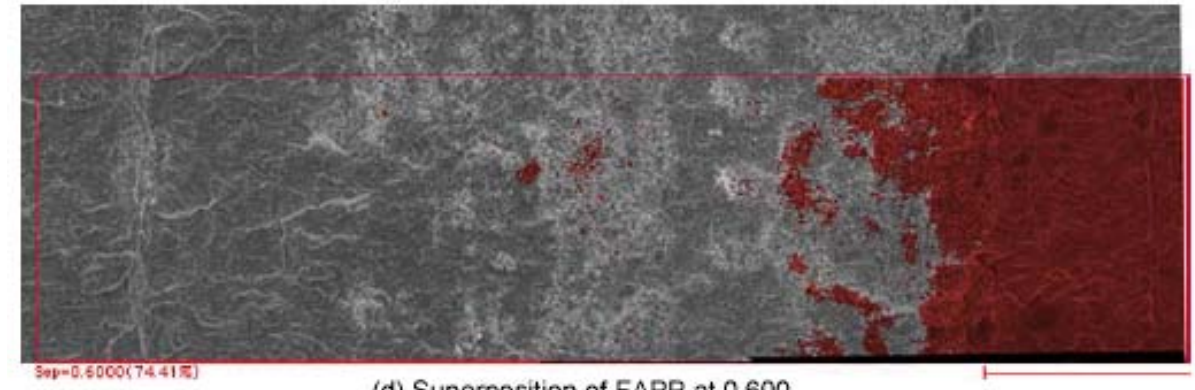

(d) Superposition of FAPP at 0.600

Figure 100. Superposition of selected FAPPs on the SEM photograph showing the correlation of interconnected placed (red areas) in FAPPs with whitish areas on SEM.

Chemical cleaning of the fracture surfaces allowed to obtain more distinct featured of crack formation and growth (Figure 101). Formation of discontinuities ahead of the crack front followed by their coalescence is clearly seen in Figure 101. The multiple data accumulated during the reported period indicated that the cracks in austenitic stainless steels advance by formation of discontinuities ahead of the crack front, followed by their coalescence. The occluded environment formed by discontinuities is filled at some point with supercritical water, 
most likely creating an aggressive media with a high concentration of impurities and shifted $\mathrm{pH}$. The formation of the thick deposits in the areas of discontinuities before their coalescence (Figure 99 and Figure 100) confirms this hypothesis.
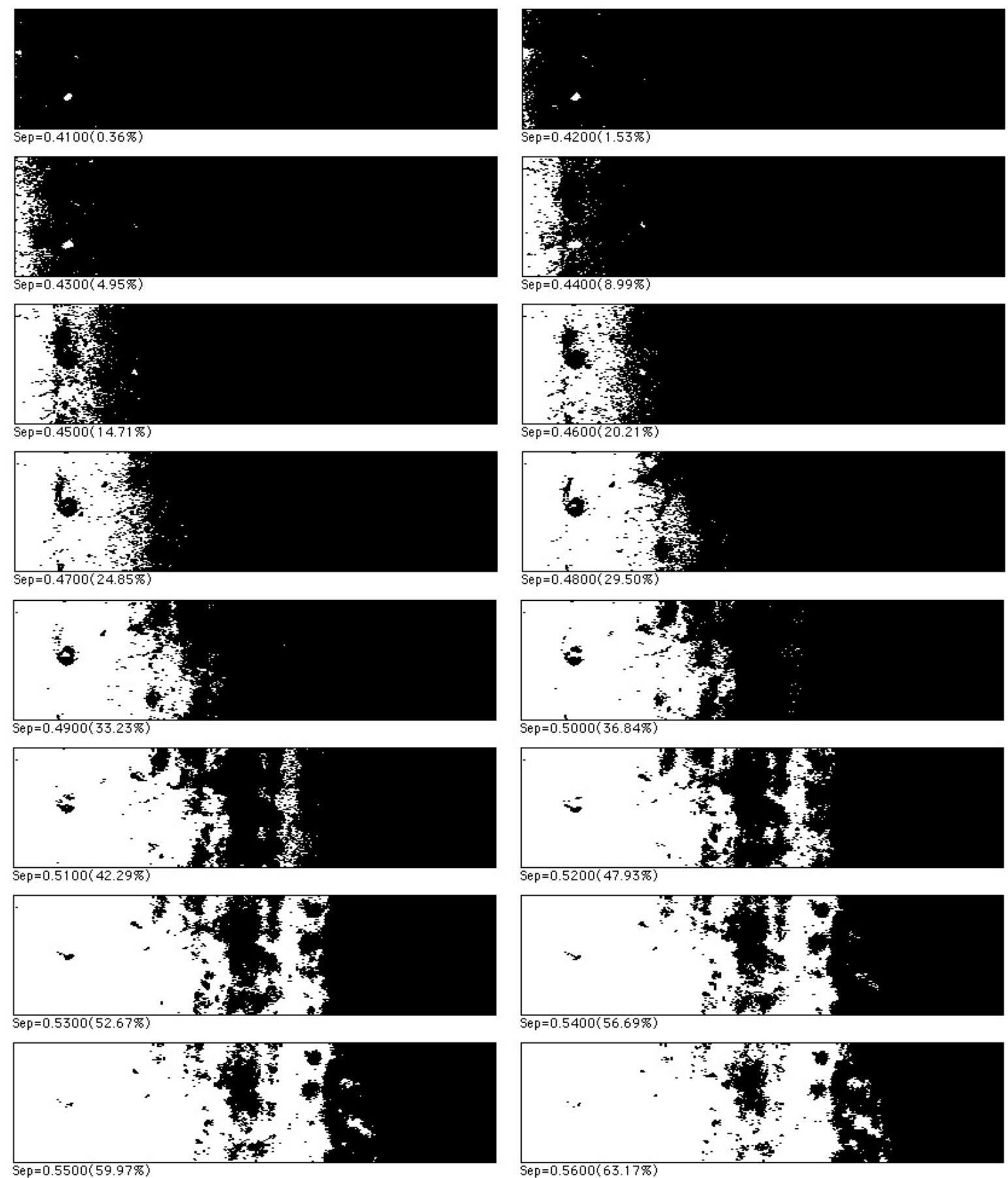

Figure 101. A series of FAPPs for chemically cleaned fracture surfaces. 
Although the crack front was not well defined, we could see some trends in the crack front movement through the series of FAPPs. However, the more precise trend in the crack front movement could be characterized by constructing the fractured area (white area) as a function of conjugate surface spacing. An example is shown in Figure 102.

The curve shows that the slope was approximately constant up to Point A, indicating that the condition of crack growth up to this point was steady and unchanged. However, at Point A, the slope changed and became a little steeper. A steeper slope suggests that the material was less resistant to the crack growth. Thus, some change occurred at Point $\mathrm{A}$ that changed the characteristics of crack growth. A possible reason for this could be the change of loading conditions: change from fatigue pre-cracking at room temperature to a supercritical water environment.

The change observed at Point B is due to the forced opening of the crack. As later shown, the crack front position observed in the FAPP at Point B corresponds to the location where surface color changed, as seen in Figure 96. The slope becomes significantly less steep after Point B, suggesting that the crack growth in this region required more plastic deformation than that in the environment.

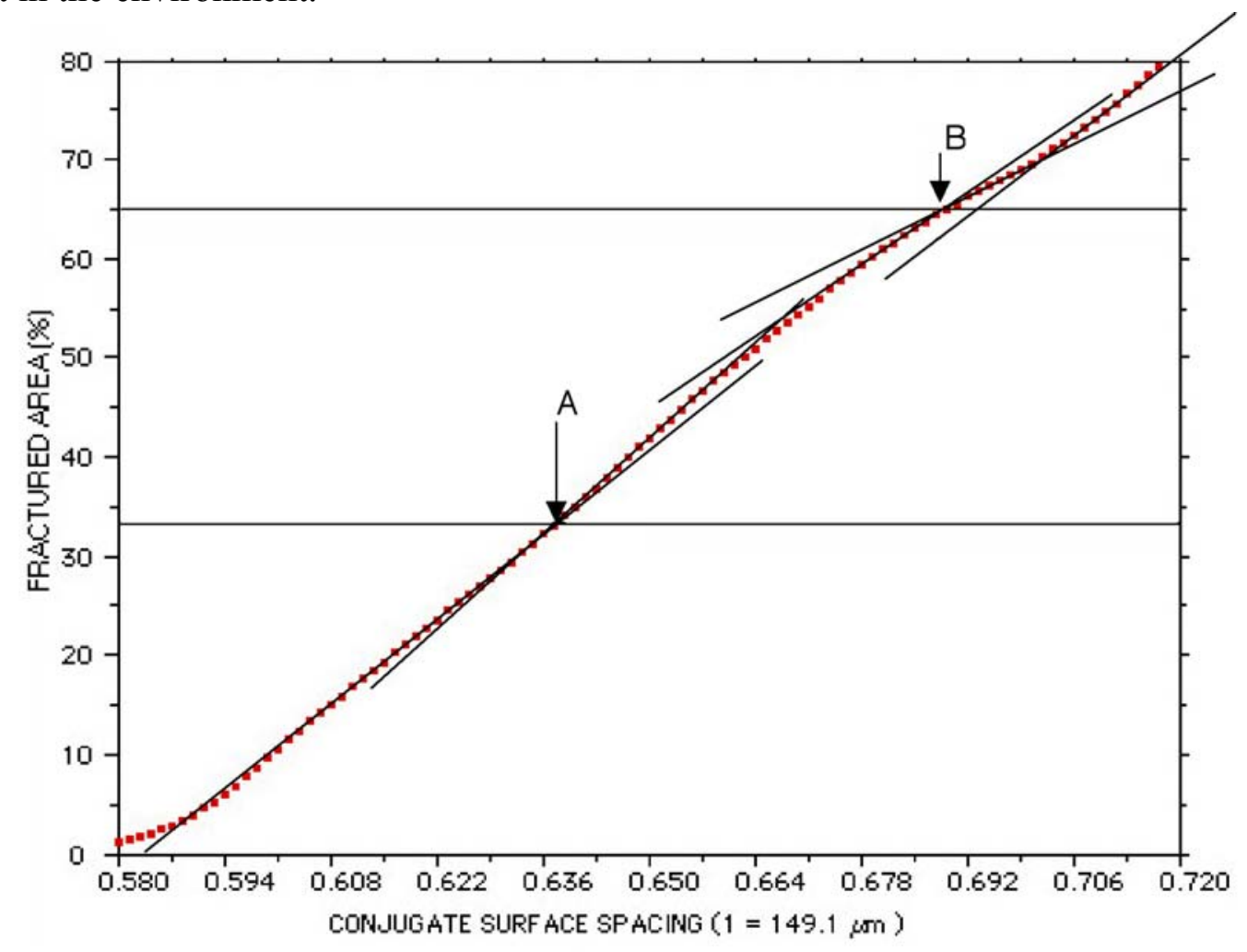

Figure 102. Fractured area increase curve as a function of conjugate surface spacing. 
Figure 103 shows the superposition of FAPP at Point A on the contrast image of Surface A shown in Figure 96. It is apparent that the dark line seen on the fracture surface corresponds to the crack front position where the slope changed. The cross sectional plot (XSP) is also shown in the figure. It is apparent that the crack growth direction changed dramatically at Point A. It is of interest to investigate why the crack growth suddenly changed direction after exposure to the different environment.

Figure 104 shows the superposition of FAPP at Point B on the contrast image of Surface A. It is clear that Point B corresponds to the state that was the end of crack growth in the supercritical environment. The XSP shows a larger overlap in the area where the forced opening took place, suggesting that the crack extension during the forced-opening operation produced larger plastic deformation at the crack tip.

These results clearly demonstrate that the FRASTA technique can identify the changes in the loading conditions and relate the changes to the locations on the fracture surface without ambiguity. Another advantage of the FRASTA is that we can examine the role of microstructure in the cracking at the super-critical environment by performing the FRASTA at a higher magnification.

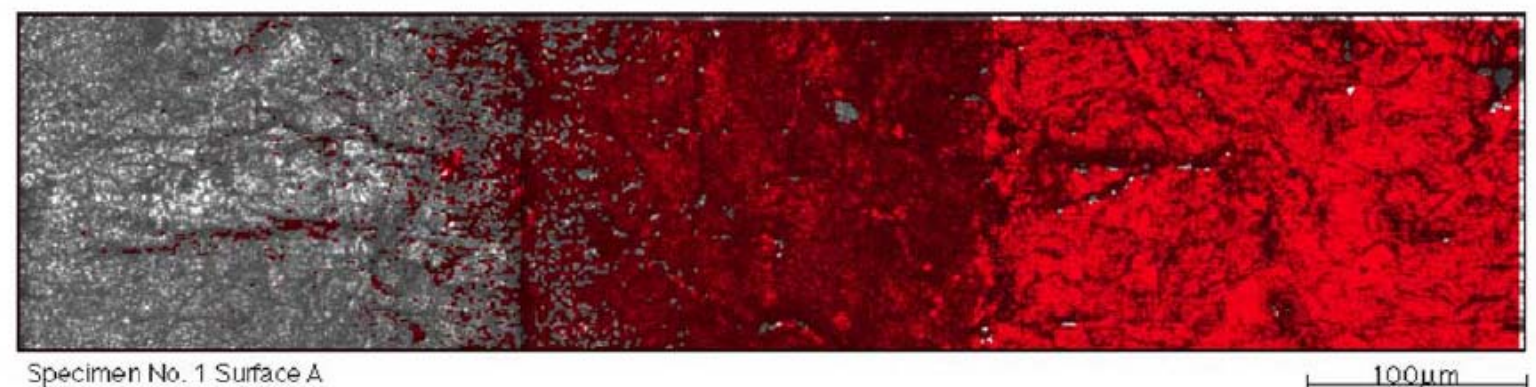

(a) Superposition of FAPP at Point A over Contrast Image

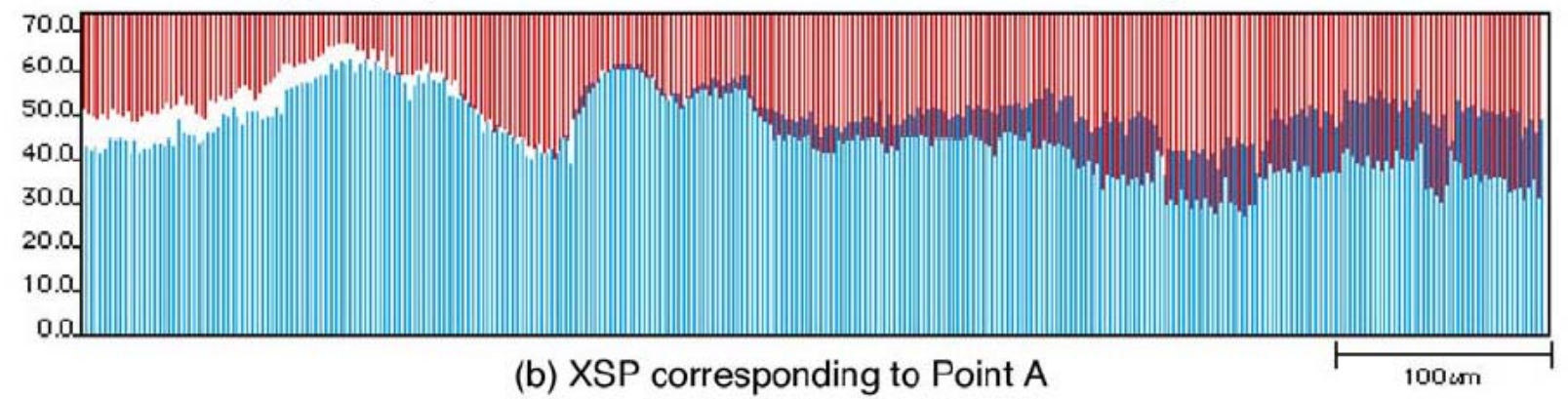

Figure 103. Superposition of FAPP at Point A over the contrast image and the corresponding XSP. 

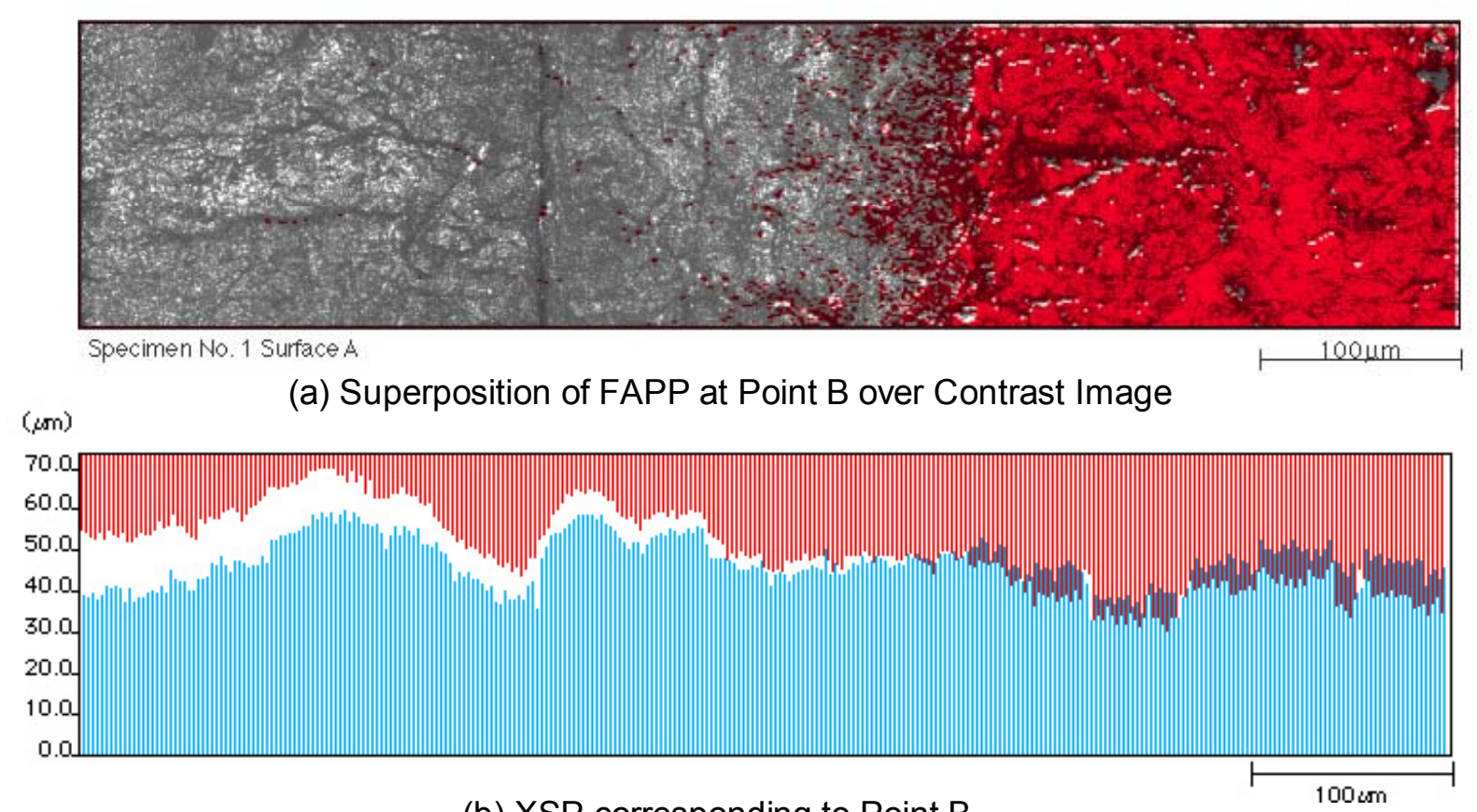

(b) XSP corresponding to Point B

Figure 104. Superposition of FAPP at Point B over the contrast image and the corresponding XSP.

\section{Estimated Crack Initiation Times}

Formation of "time marks" on the fracture surfaces associated with reactor shut down/ start up cycles (examples are shown in Figure 94 and Figure 95) allowed us to estimate crack initiation times. We defined an event of "crack initiation" as coalescence of discontinuities accumulated ahead of the crack front observed at FAPPs (an example is shown in Figure 101). Two nearest time marks defined exposure interval (between two re-starts) when crack initiation took place.

Crack initiation times (Figure 105 shows results for highly oxidizing conditions) have been estimated using linear interpolation of crack extension between two closest time marks (in other words, assuming constant crack growth rate between two re-starts). High uncertainties of the estimated crack initiation times ( $\sim$ factor of 2 ) are associated with not clearly defined fronts of real cracks. Some hidden uncertainty may be associated with assumption of the constant crack growth rates between re-starts. Estimated crack initiation time for 304 stainless steel was found to be on the order of tens of hours. A several times longer crack initiation time was 
obtained for 316 stainless steel. Crack initiation time for 347 stainless steel was an order of magnitude longer than for SS316. Quantitative estimation of crack initiation times for low oxidizing conditions was not possible because of much lower crack growth rates and high uncertainties in localization of crack initiation events.

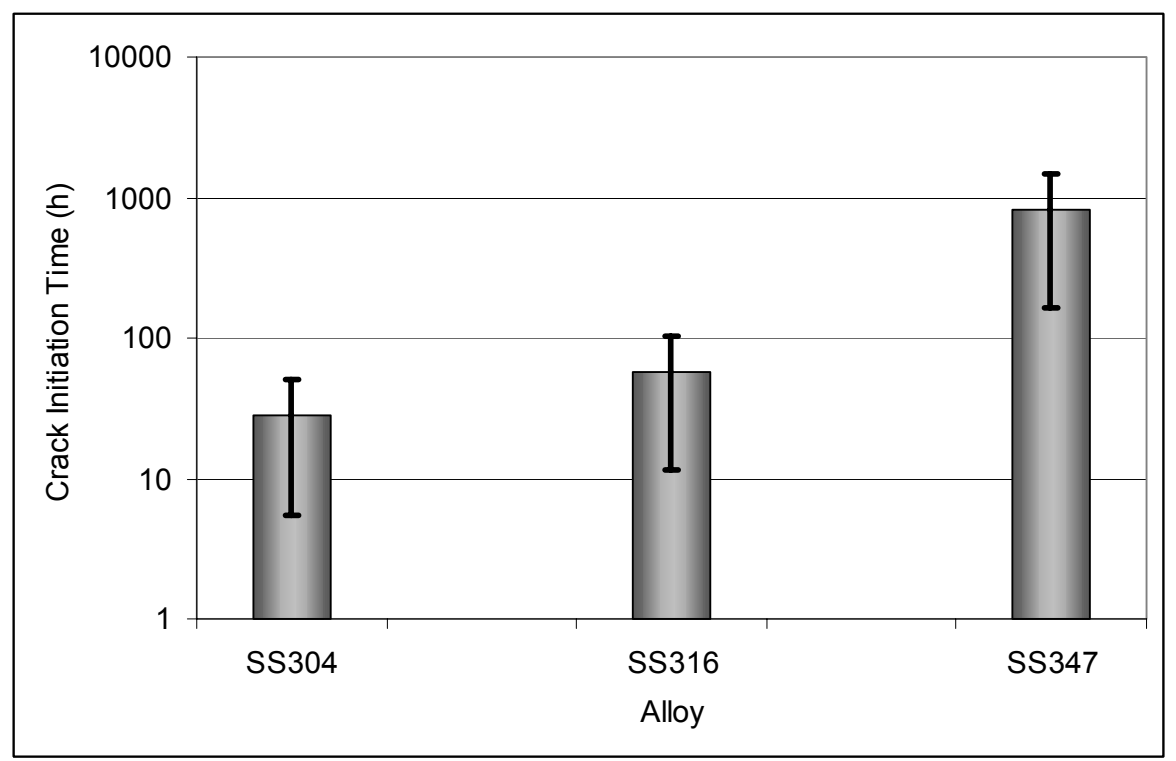

Figure 105. Estimated crack initiation times for austenitic stainless steels

\section{Estimated Crack Growth Rates}

Crack extension vs. time information was obtained using SEM and FRASTA images and "time marks" using the following procedure. The distance between each time mark was measured using SEM images (Figure 94b). Since the time between any two "time marks" is known from the duration of operation between shutdowns, crack extension vs. time information may be obtained. Figure 106 shows crack propagation in stainless steels exposed to deoxygenated (100 ppb O2) supercritical water. For 304 stainless steel, the time dependence of crack growth follows closely a second order curve. Accordingly, the crack growth rate decreased linearly as the load decreased with the increase of the crack length. Total extension of the crack, as measured using the SEM image in Figure 94b, is about 300 microns. A noticeable slow down in crack growth was observed for SS304 and SS347 (Figure 106). Uncertainties in estimated crack growth are about factor of 2 at initial stage and about $30 \%$ for final stage (at the end of $\sim 1000$ hours of exposure). 


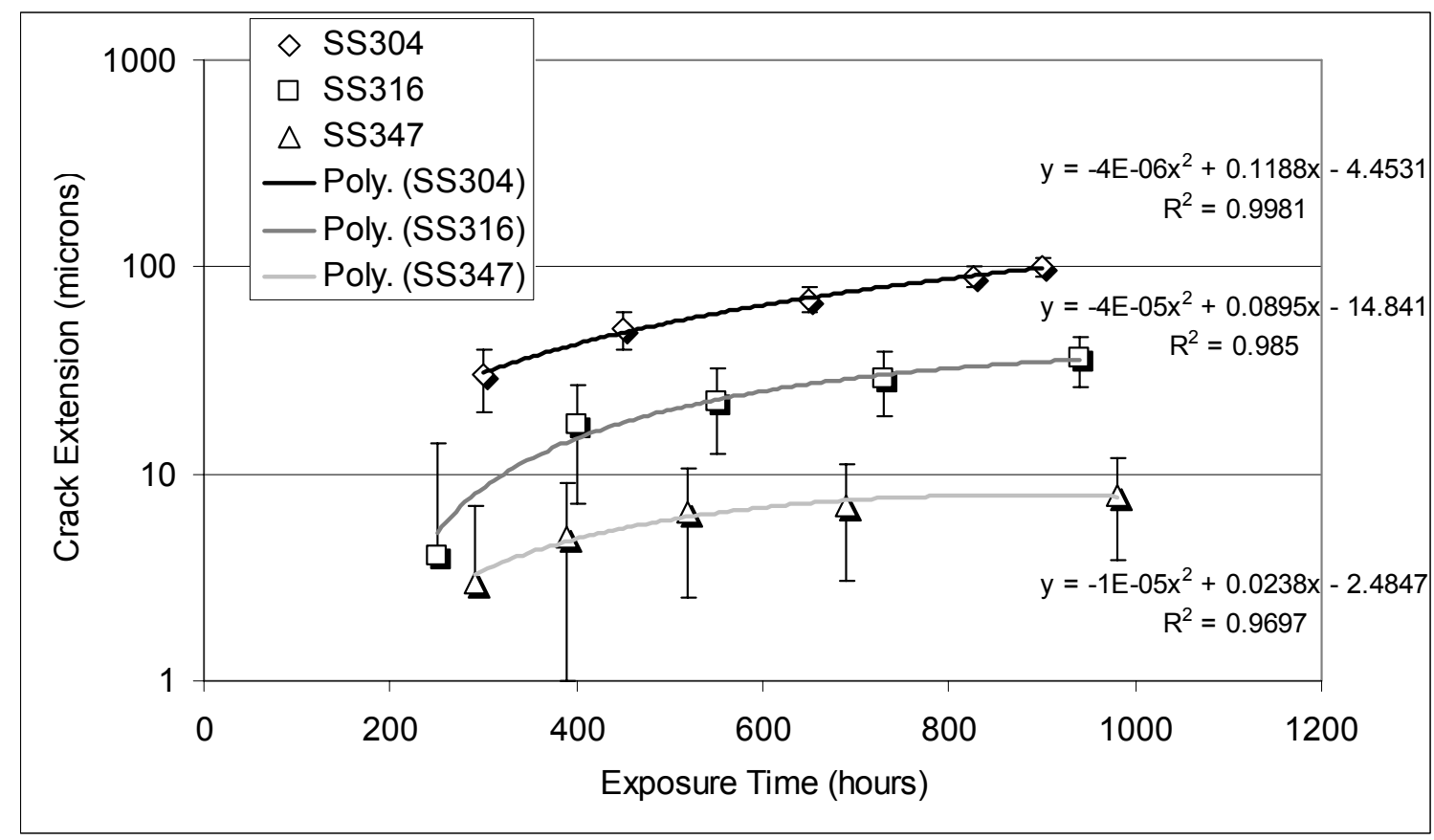

Figure 106. Crack growth in austenitic stainless steels

In order to estimate crack growth rates, we approximated crack growth data in Figure 106 with second order trend lines. Correlation coefficient in all cases is very close to 1.0. Empirical equations for crack extensions $a(t)$ are also shown in Figure 106. Crack growth rates, which have been estimated as $d a / d t$, are shown in Figure 107.

The magnitude of crack growth rates was found to be surprisingly low $\left(<10^{-8} \mathrm{~cm} / \mathrm{s}\right)$, even for SS304, approaching creep limit for SS347. From a reactor design point of view these results should be interpreted only in a qualitative manner, because oxidizing conditions formed under mixed neutron+gamma radiation may be more complex than the ones simulated in these out of pile tests. The objective of the present study was only to rank candidate structural materials against their susceptibility to SCC. Clearly, in order to obtain experimental crack growth rate data for SCWR design analysis, in-pile experiments are needed. However, the results of the present work will allow significantly reduce number of candidate alloys for in-pile tests.

Slow down in the crack growth rates with time may result partially from test conditions (load relaxation of pre-loaded specimens) and partially because of the electrochemical processes as it was previously demonstrated for sub-critical temperatures for austenitic (mostly SS304) steels. 


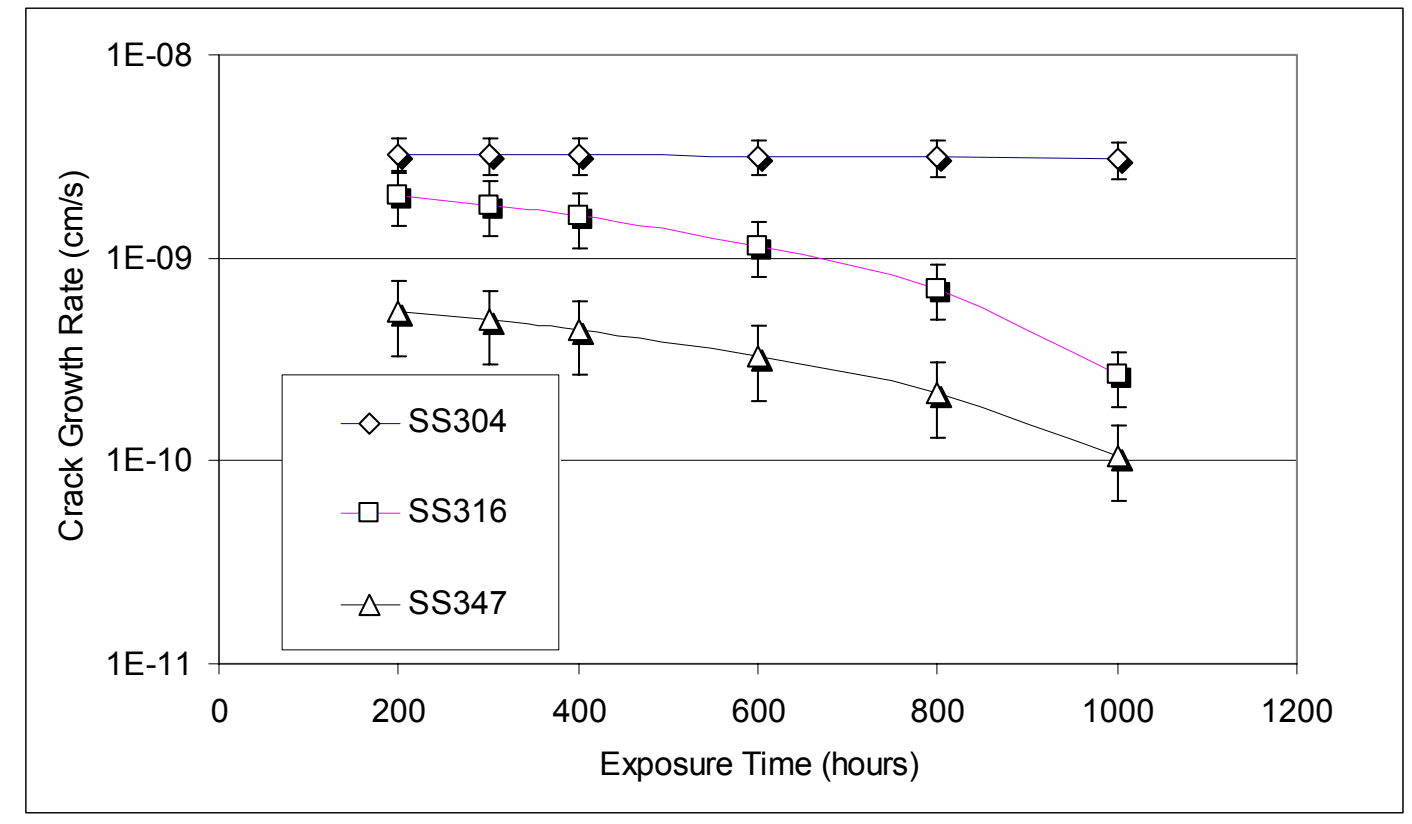

Figure 107. Crack growth rates for austenitic stainless steels

\section{Ranking Materials against Susceptibility to Environmentally Assisted Degradation}

Finally, candidate austenitic steels (SS304, SS316, and SS347) were ranked against their susceptibility to environmentally assisted degradation based on film stability parameters obtained from CDE measurements. The stability of the oxide films was characterized by the following three parameters: (1) CER, (2) charge transfer resistance, and (3) diffusion resistance. These three measurable parameters define film stability - the rate of charge and mass transfer across the oxide film.

Our approach to describe environmentally assisted degradation is to accept that the transport of species through the oxide films on the crack walls is one of the rate-controlling factors for crack growth. Assuming that the transport of ionic defects through the oxide film on the crack walls contributes to the crack growth rate, a correlation should exist between the crack growth rate and the oxide film properties. Generally, the more ionic defects an oxide film contains, and thus the less stable the film is, the faster the transport of ionic species takes place through the film.

The resistance to both electronic and ionic charge carries was observed to be the lowest for SS304 and the highest for SS347, with SS316 being between the two (Figure 108a). A convenient way to rank materials against their susceptibility to environmentally assisted 
degradation is to define the relative stability of the oxide films by selecting the worst performing material (SS304 in this case) as a baseline.

Results of SCC tests conducted during reported period with the same materials showed that SS347 is less susceptible for SCC than SS316 and much less susceptible than SS304 (Figure 107). Comparison of these observations with transport properties of the oxide films (Figure $108 \mathrm{a}$ and b) clearly indicates that we were able to observe experimentally a correlation between measurable oxide film properties and the susceptibility of austenitic steels to environmentally assisted degradation, including SCC. Experimental proof of the existence of such correlation was one of the major goals of the present work.

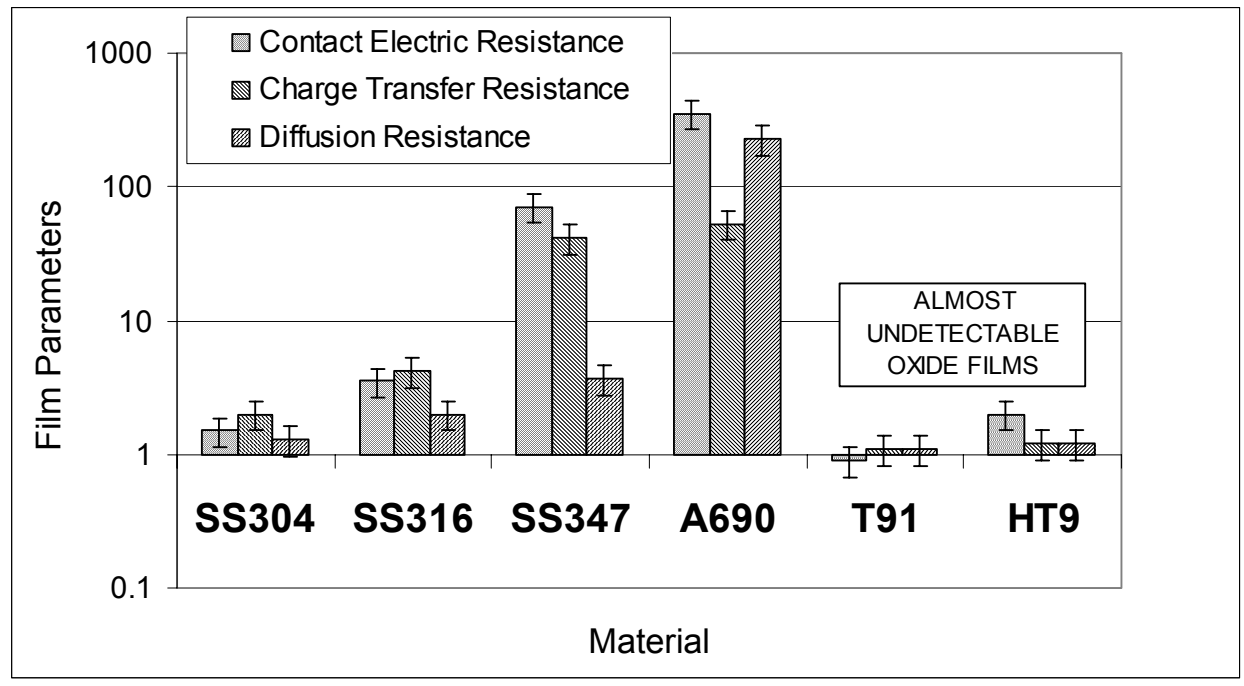

(a)

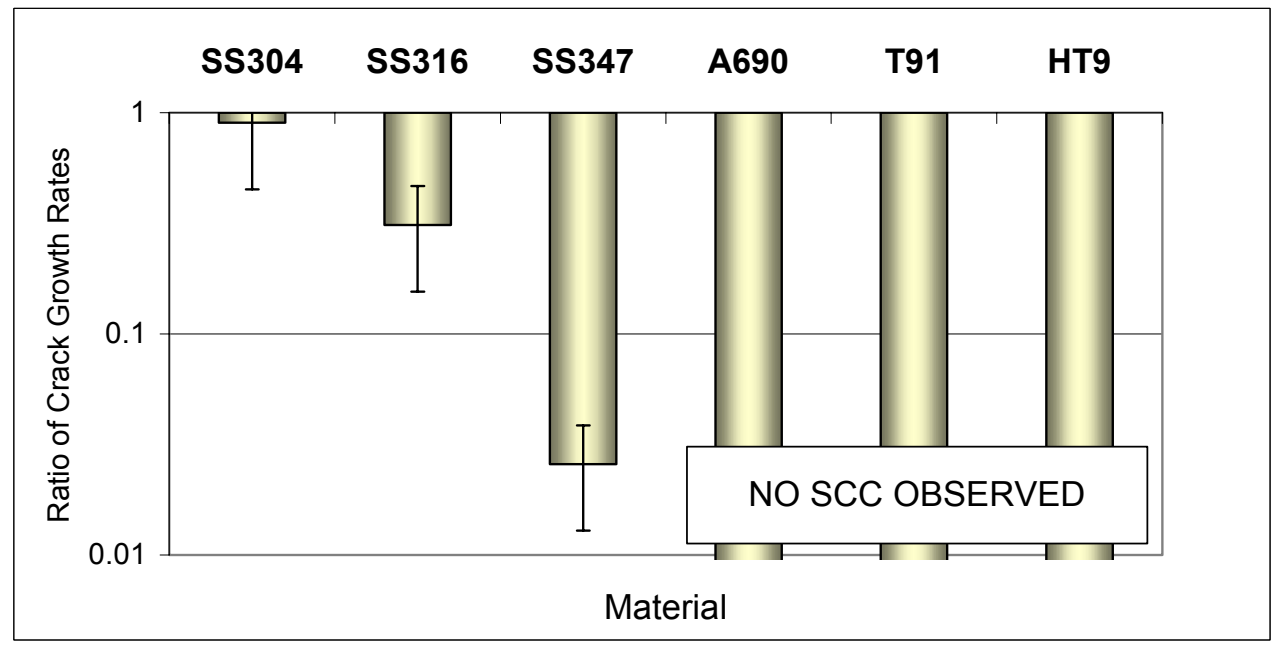

(b)

Figure 108. Transport properties of the oxide films (a) and crack growth rates (b) relative to SS304. 
Collected data on transport properties of the oxide film allowed estimation of crack growth for a representative set of operating conditions anticipated in the heat transport circuit of SCWR with reduced number of direct SCC measurements. In our approach we rely on the evolution model of crack propagation as a moving boundary value problem, where material dissolution advances the boundary exposed to the corrosive environment. A controlling mechanism for the boundary advancement is the rupture of a brittle corrosion-protective film, which is continually building-up (repassivation of bare metal) along the corroding surface. The rate of boundary evolution is a function of the degree of the protective film damage, caused by mechanical straining. Interaction between the surface deformation and the protective film is primarily responsible for the dissolution localization along a narrow surface region, such that a crack is formed from a pit and the crack shape is maintained during the evolution.

Effect of repassivation on the crack growth rates was quantified for 347 stainless steel, pure Ni, and nickel based alloy A690. Repassivation time was obtained from the measured data on repassivation kinetics. According to (Eq.28) crack growth rate is decreasing as bare metal formed during a slip step repassivates. While $v_{0}$ depends on the electrochemical dissolution of a bare metal, repassivation term $\left(\frac{t_{0}}{t}\right)^{p}$ depends on the repassivation rate and stability of the oxide film. Repassivation data such as shown in Figure 109 have never been measured in-situ neither for sub- no for super-critical temperatures. Availability of such data opens a new and more cost effective way of ranking candidate structural materials against susceptibility to stress corrosion cracking.

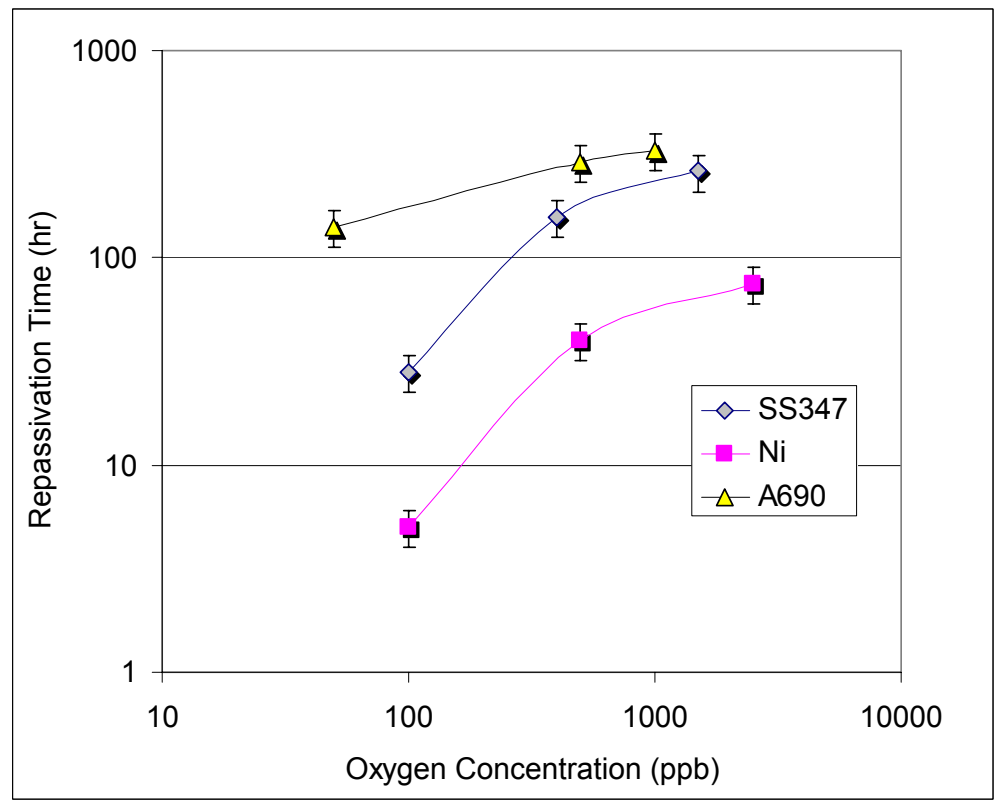

Figure 109. Repassivation time of SS347, pure nickel and alloy 690. 
As and example, Figure 110 demonstrates crack growth rate decrease with time for several (material, water chemistry) combinations during a single slip-dissolution-repassivation step. Clearly, susceptibility of all materials to SCC decreases as concentration of the dissolved oxygen decreases (crack rate decreases faster with time). Effect of oxidizing conditions on SCC is important factor in selection of structural materials since components located in high neutron and gamma fluxes will always be exposed to high levels of oxidizing species such as radiolytically produced hydrogen peroxide and oxygen (as a product of $\mathrm{H}_{2} \mathrm{O}_{2}$ decomposition), even if hydrogen is injected to suppress oxygen level.

Traditional approach, which relies only on the repassivation time and ignores the impact of the stability of the oxide films may lead to conservative or even misleading conclusions. This statement is illustrated in Figure 110. Figure 110a depicts crack repassivation factor (Eq.28) without film stability taken into account (traditional approach). Figure 110b depicts crack repassivation factor for the same (material, environment) combinations with oxide film stability taken into account by replacing exponent $p=0.5$ in Eq.28 with $p=$ function(oxide film stability). In the first case (Figure 110a) a material of choice would be SS347. In the second case (Figure 110 b) alloy 690 should be considered as an alternative.

Design analysis of SCWR would require even more complex considerations, such as the effect of stress on the rupture of the oxide film. Data on crack extension (Figure 111) demonstrate how timing of film rupture may affect the choice of material. For example, if we consider a case of low oxidizing conditions (50-100 ppb of dissolved $\mathrm{O}_{2}$ ) SS347 would be a better choice if film rupture occurs during $\mathrm{t}=200-300$ hours and A690 would be a better choice if loading conditions are such as that the film rupture occurs at a later time (after 500 hours in this case).

Finally, we have to emphasize again, the SCWR design analysis should rely on data similar to the one presented in this report, but obtained in in-pile experiments under realistic oxidizing conditions of supercritical water radiolysis in a combined field of neutron+gamma irradiation. The objective of the present study was to establish a scientific basis for more reliable and, at the same time, more cost effective way of selecting structural materials for heat transport circuit of SCWRs. The reported results demonstrate, in our opinion, that this objective was met. 


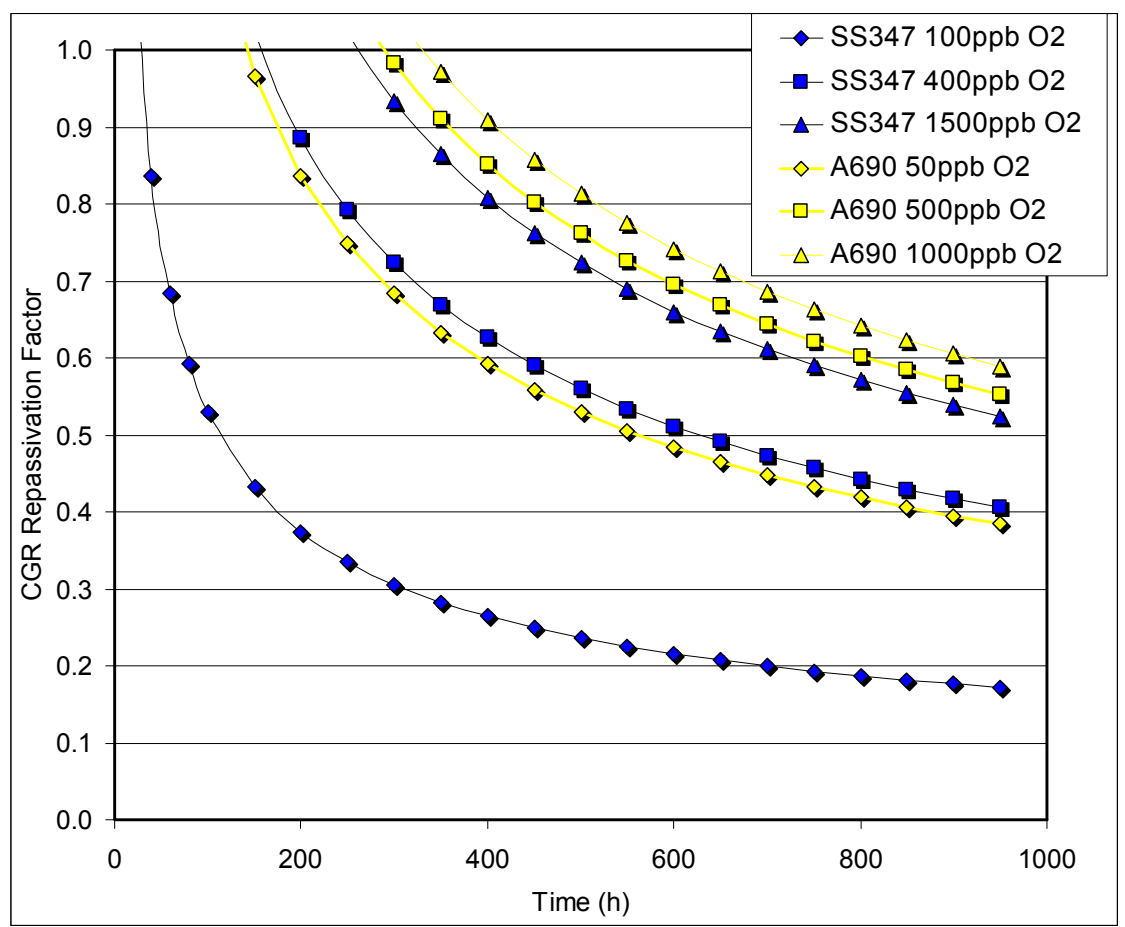

(a)

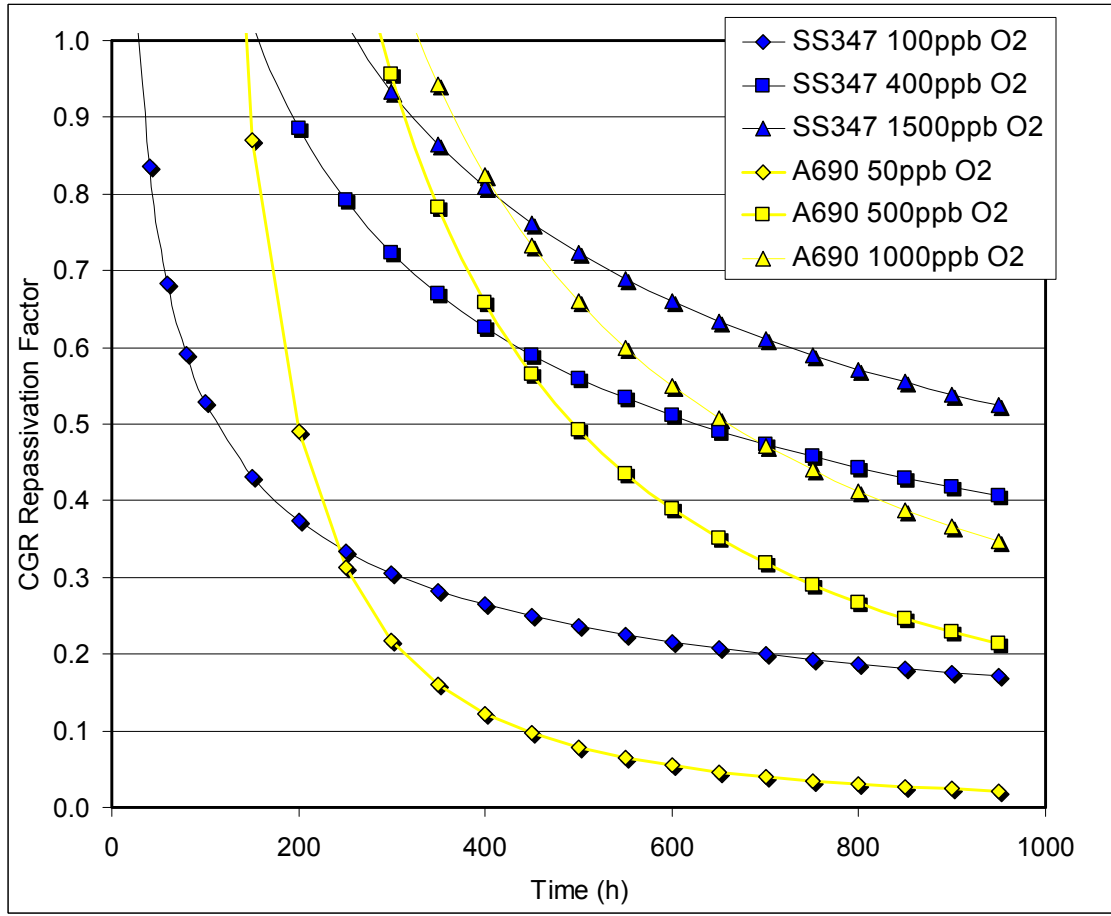

(b)

Figure 110. Crack growth rate decrease due to repassivation for SS347 and alloy A690: (a) without considering oxide film stability and (b) with exponent in repassivation term proportional to oxide film stability. 


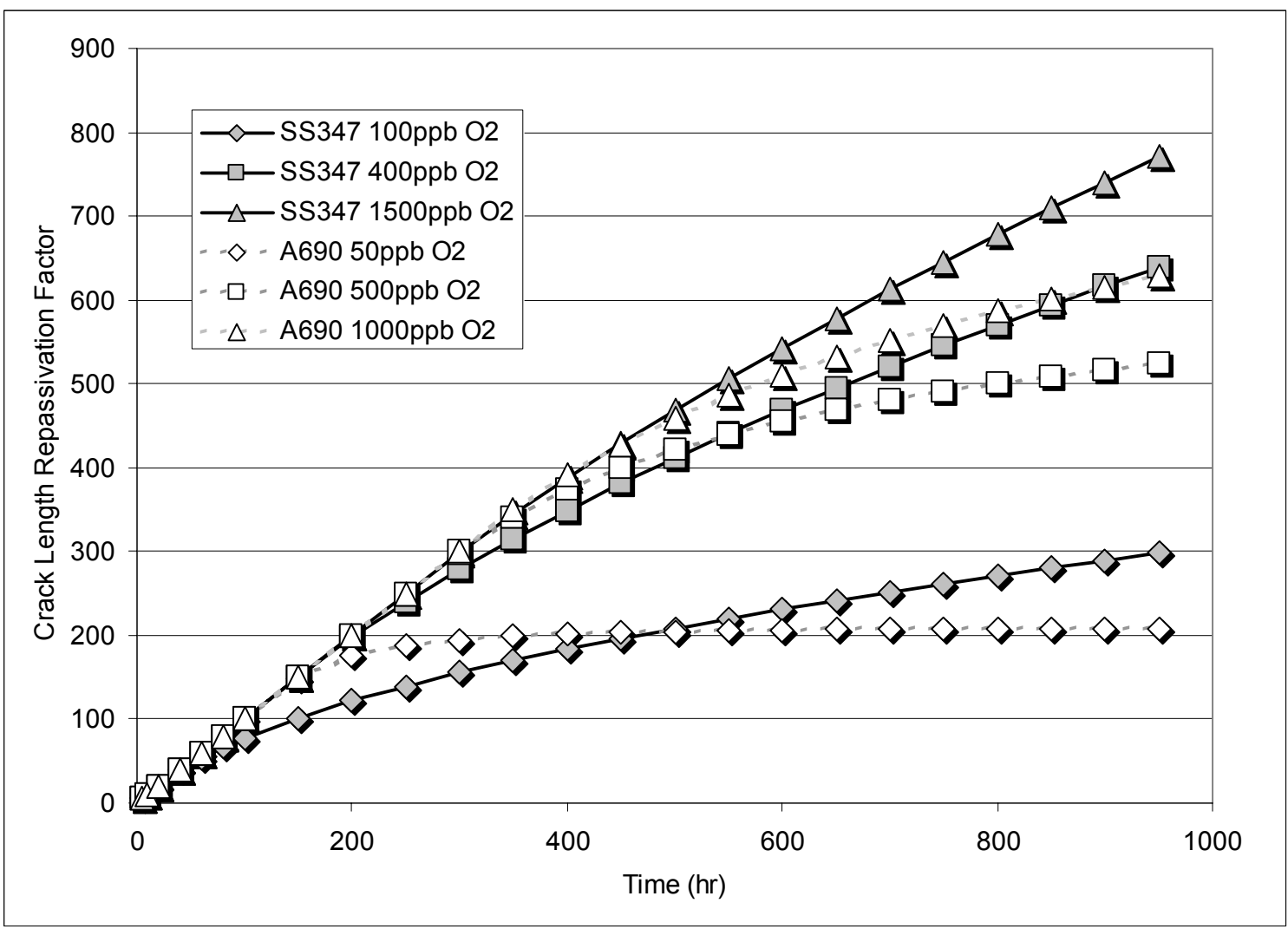

Figure 111. Crack growth in candidate structural materials under low, medium, and high oxidizing conditions 


\section{PROJECT BUDGET}

Project was completed on time and within the budget. During the first year of the project spending was under the budget, because the majority of the efforts was focused on the developments of the test system. Periods of design activity were mixed with waiting periods (no charges) for custom made parts to arrive. Once the test system was assembles and experimental work commenced, the spending rate was increasing steadily. A carryover from the first year allowed us to stay within the budget during second and third years characterized by intensive experimental activity and analysis of large amount of the experimental information.

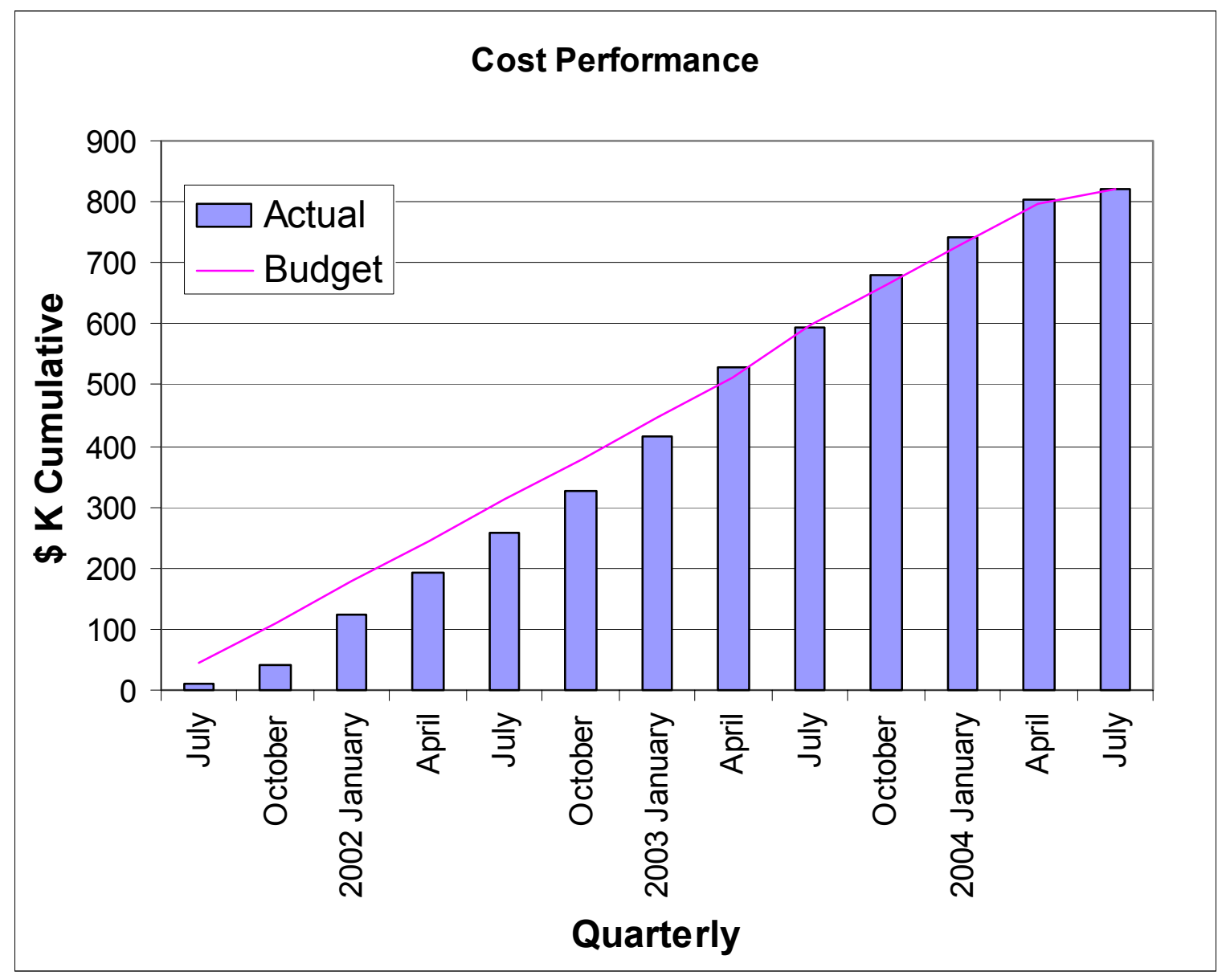

Figure 112. Project quarterly cost performance 


\section{CONCLUSIONS AND FUTURE WORK}

The major accomplishments during the course of work on this project follow:

- A test system for electrochemical and fracture mechanics studies in supercritical water was designed and built. The system includes an electrochemical part and a fracture mechanics part. The electrochemical part includes a CDE arrangement for in-situ studies of oxide films at supercritical temperatures. The fracture mechanics part includes fracture mechanics specimens for stress corrosion cracking studies and the loading system.

- Systematic experimental data on the charge and mass transfer properties of the oxide films were obtained for a representative set of candidate structural materials. CDE measurements in three configurations (CER, CEI, and TLEC) provided in situ information on the rate of transport of electronic and ionic charge carriers in the oxide film and at the film/supercritical water interface.

- Kinetic parameters of the charge and mass transfer processes in the oxide films and at the film/supercritical water interface determined from CDE measurements were in good agreement with independent results obtained at VTT. A set of kinetic parameters, which describe transport processes, was defined. This set includes interfacial rate constants for generation and consumption of ionic current carriers, the diffusion coefficient of ionic point defects (vacancies or interstitials), the diffusion coefficient of electronic current carriers, the field strength in the oxide, the thickness of the layer in which point defect transport occurs, and the polarizability of the oxide/supercritical water interface. Work on deriving kinetic data for oxide films formed on candidate structural materials from CDE measurements is in progress.

- The first group of candidate structural materials (austenitic stainless steels SS304, SS316, and SS347) was ranked against their susceptibility to environmentally assisted degradation. SCC was not observed for nickel alloys, alloy T91 and HT9. The ranking was based on the in situ data on film stability parameters (or film transport properties) measured with a CDE arrangement designed at SRI.

- Comparison of these observations and the results of CDE measurements at SRI clearly indicated that we were able to observe experimentally a correlation between measurable oxide film properties and the susceptibility of austenitic steels to environmentally assisted degradation. Experimental proof of the existence of such correlation is one of the major goals of the present work. One of the major results of the present work is the experimentally proven ability of the economical CDE technique to supply in situ data for ranking candidate structural materials for SCWR.

- The morphology and thickness of the oxide films were examined with SEM. The chemical composition of the films was measured with the EDX technique. Direct measurements of film thickness were in good agreement with in situ film thickness measurements using the $\mathrm{CDE}$ technique. Oxide films were found to be enriched in $\mathrm{Cr}$ 
(relative to the base metal). The $\mathrm{Cr}$ concentration was found to decrease in the outer surface layer of the film.

- During the reporting period, we also estimated the potential use of the CDE technique for building in situ sensors for monitoring water chemistry in the heat transport circuit of Generation-IV SCWRs. Two types of sensors are envisioned: (1) a monitor to measure oxide film stability and, accordingly, the susceptibility of the in-vessel structural materials to various forms of environmentally assisted degradation, and (2) a water conductivity sensor working in a wide temperature range-from room temperature to supercritical temperatures.

- Multiple data on the crack front movement obtained from FRASTA confirmed that the cracks in austenitic stainless steels advance by formation of discontinuities ahead of the crack front, followed by their coalescence. The occluded environment formed by discontinuities becomes filled with supercritical water, most likely creating an aggressive medium. Formation of the thick deposits in the areas of discontinuities confirmed this hypothesis.

- Experimental data obtained during the reporting period allowed estimation of the crack growth rates for austenitic stainless steels in supercritical water. This group included SS304 as a "baseline" material and SS316 and SS347 as candidate materials. Our results indicated that SS347 is expected to have an order-of-magnitude lower crack growth rate than SS304 and that the rate for SS316 is expected to be several times lower than that for SS304.

- A combined effect of film repassivation rate and stability of the oxide film on SCC behavior and life-time of structural materials was demonstrated for a representative set of oxidizing conditions. An approach for quantitative estimation of materials lifetime depending on (material, environment) combination was suggested.

Should NERI program be revitalized by a sufficient funding, we would consider submitting a joint (universities, national laboratories and research centers) proposal to address the following SCWR materials reliability issues:

- In-pile experiments in a research reactor to study oxide film formation on candidate structural materials coupled with a limited number of in-pile SCC tests.

- In-situ measurements of the radiolytic yields of species in supercritical water under combined neutron and gamma irradiation.

- Development of a validated radiolysis model for reliable prediction of the oxidizing conditions, distribution of the electrochemical corrosion potential, and estimation of crack growth rates in SCWR heat transport circuit.

- Development of the CDE-based advanced in-situ instrumentation for monitoring water chemistry conditions and the state of the structural materials under timedependent operating conditions. 
Finally, we would like to thank NERI program reviewers and administrators for selecting this project for funding and providing an opportunity to advance fundamental understanding of materials reliability in supercritical water. 


\section{REFERENCES}

1. J. Buongiorno and P.E. MacDonald, Supercritical Water Reactor (SCWR) Progress Report for the FY-03 Generation-IV \&D Activities for the Development of the SCWR in the U.S., INEEL/EXT-03-01210, September 30, 2003.

2. J. Buongiorno, W. Corwin, P.E. MacDonald, L. Mansur, R. Nanstad, R. Swindeman, A. Rowcliffe, G. Was, D. Wilson, and I. Wright, Supercritical Water Reactor (SCWR) Survey of Materials Experience and $R \& D$ Needs to Assess Viability, INEEL/EXT-0300693 (Rev. 1), September 2003

3. I. Balachov, I. Jayaweera, T. Kobayashi, P. Jayaweera, Fundamental Understanding of Crack Growth in Structural Components of Generation IV Supercritical Light Water Reactors, NERI Project 1-130, Annual Report, September 2002.

4. I. Balachov, I. Jayaweera, T. Kobayashi, P. Jayaweera, M. Bojinov, P. Kinnunen, T. Laitinen, K. Mäkelä, and T. Saario, Crack Growth in Structural Components of Generation IV Supercritical Light Water Reactors, Proc. Int. Conf. Adv. Power Plants, Córdoba, Spain, May 4-7, 2003, Paper 3266

5. M. Bojinov, J. Hinttala, T. Laitinen, E. Muttilainen, K. Mäkelä, A. Reinvall, T. Saario. and S. Suksi, Development of electrochemical techniques to study oxide films on construction materials in high temperature water, 1998 JAIF International Conference on Water Chemistry in Nuclear Power Plants, Kashiwazaki, Japan, 1998.

6. I. Balachov, M. Bojinov, T. Laitinen, E. Muttilainen, K. Mäkelä, A. Reinvall, T. Saario and P. Sirkiä, Correlations between the stability of oxide films in simulated BWR crack conditions, SO42- enrichment and cracking susceptibility, VIIIth International Conference of Water Chemistry of Nuclear Reactor Systems, Bournemouth, UK, 2000.

7. A. Toivonen, E. Muttilainen, Effects of BWR coolant sulphate ion concentration on stress corrosion crack propagation in AISI 304, in AISI 316NG and in Inconel Weld Metals 82 and 182 (to be published).

8. P. L. Andresen, Observation and prediction of the effects of water chemistry and mechanics on environmentally assisted cracking of Inconels 182 weld metal and 600, Corr. Sci. 44, No. 6, 1988, p. 376.

9. A. McMinn, and R. A. Page, Stress corrosion cracking of Inconel alloys and weldments in high-temperature water - the effects of sulfuric acid addition, Corrosion Vol. 44, No. 4, 1988, p. 239.

10. FINNUS. The Finnish Research Programme on Nuclear Power Plant Safety. VTT, Espoo, 2000.

11. Epelboin, C. Gabrielli, M. Keddam, and I. Takenouti, in Comprehensive Treatise on Electrochemistry (Edited by J.O'M. Bockris, B.E. Conway, and E.B. Yeager), Plenum Press, New York (1981), Vol. 4, p. 151

12. D. D. Macdonald and M.C.H. McKubre, in Impedance Spectroscopy (Edited by J. Ross Macdonald) John Wiley \& Sons, New York (1987), p. 281. 
13. M. Keddam, J.-F. Lizee, C. Pallotta, and H. Takenouti, J. Electrochem. Soc. 131 (1984) 2016.

14. D.D. Macdonald and S.I. Smedley, Electrochim. Acta 33 (1990) 1949.

15. E.B. Castro, Electrochim. Acta 39 (1994) 2117.

16. X.-P. Guo, Y. Tomoe, H. Imaizumi, and K. Katoh, J. Electroanal. Chem. 445 (1998) 95.

17. C. Gabrielli, M. Keddam, and H. Takenouti, Electrochim. Acta 35 (1990) 1553.

18. Annergren, M. Keddam, H. Takenouti, and D. Thierry, Electrochim. Acta 41 (1996) 1121.

19. Annergren, M. Keddam, H. Takenouti, and D. Thierry, Electrochim. Acta 42 (1997) 1595.

20. C. Gabrielli, M. Keddam, F. Minouflet, and H. Perrot, Electrochim. Acta 41 (1996) 1217.

21. C. Gabrielli, M. Keddam, N. Nadi, and H. Perrot, J. Electroanal. Chem. 485 (2000) 101.

22. R. Antaño-Lopez, M. Keddam, and H. Takenouti, Electrochim. Acta 46 (2001) 3611.

23. C. Deslouis, M.M. Musiani, B. Tribollet, and M.A.Vorotyntsev, J. Electrochem. Soc. 142 (1995) 1902.

24. M. A. Vorotyntsev, C. Deslouis, M. M. Musiani, B. Tribollet, and K. Aoki, Electrochim. Acta 44 (1999) 2105.

25. J.B. Memet, P. Girault, R. Sabot, C. Compere, and C. Deslouis, Electrochim. Acta 47 (2002) 1043.

26. J. Maier, Progr. Solid State Chem. 23 (1995) 171.

27. M. Liu, J. Electrochem. Soc. 144 (1997) 1813.

28. P. Santos, R. Z. Domingues, and M. Kleitz, J. Eur. Ceram. Soc. 18 (1998) 1571.

29. J.J. Vermoyal, L. Dessemond, A. Hammou, and A. Frichet, J. Nucl. Mater. 288 (2001) 297.

30. J.J. Vermoyal, A. Hammou, L. Dessemond, and A. Frichet, Electrochim. Acta 47 (2002) 2679.

31. S. Song and P. Xiao, Mater. Sci. Eng. A323 (2002) 27.

32. S. Song and P. Xiao, J. Mater. Sci. 38 (2003) 499.

33. Betova, M. Bojinov, E. Lankinen, and G. Sundholm, J. Electroanal. Chem. 472 (1999) 20.

34. B.W. Johnson, D.C. Read, P. Christensen, A. Hamnett, and R.D. Armstrong, J. Electroanal. Chem. 364 (1994) 103.

35. T. Komura, S. Goishihara, T. Yamaguti, and K. Takahasi, J. Electroanal. Chem. 456 (1998) 121.

36. M. Bojinov, T. Laitinen, K. Mäkelä, and T. Saario, J. Electrochem. Soc. 148 (2001) B243.

37. Betova, M. Bojinov, A. Englund, G. Fabricius, T. Laitinen, K. Mäkelä, T. Saario, and G. Sundholm, Electrochim. Acta 46 (2001) 3627.

38. V.A. Marichev, Surf. Sci. Reports 44 (2001) 51. 
39. T. Saario, T. Laitinen, and J. Piippo, Mater. Sci. Forum 289-292 (1998) 193.

40. B. Beverskog, M. Bojinov, A. Englund, P. Kinnunen, T. Laitinen, K. Mäkelä, T. Saario, and P. Sirkiä, Corros. Sci. 44(2002) 1901.

41. H. Göhr, Ber. Bunsenges. Phys. Chem. 85 (1981)274.

42. C.A. Schiller and W. Strunz, Electrochim. Acta 46 (2001) 3619.

43. B. Beverskog, M. Bojinov, P. Kinnunen, T. Laitinen, K. Mäkelä, and T. Saario, Corros. Sci. 44 (2002) 1923.

44. D.D. Macdonald, S.R. Biaggio, and H. Song, J. Electrochem. Soc. 139 (1992) 170.

45. D.D. Macdonald, J. Electrochem. Soc. 139 (1992) 3434.

46. D.D. Macdonald, Pure Appl. Chem. 71 (1999) 951.

47. J. Fleig and J. Maier, Solid State Ionics 94 (1997) 199.

48. M. Bojinov, G. Fabricius, T. Laitinen, K. Mäkelä, T. Saario, and G. Sundholm, Electrochim. Acta 45 (2000) 2029.

49. M. Bojinov, G. Fabricius, P. Kinnunen, T. Laitinen, K. Mäkelä, T. Saario, and G. Sundholm, J. Electroanal. Chem. 504 (2001) 29.

50. X.-P. Guo, Y. Tomoe, H. Imaizumi, and K. Katoh, J. Electroanal. Chem. 445 (1998) 95.

51. B. Krishnamurthy, R.E. White, and H.J. Ploehn, Electrochim. Acta 47 (2002) 3375.

52. M.A. Vorotyntsev, J.-P. Badiali, and E. Vieil, Electrochim. Acta 41 (1996) 1375.

53. M.A. Vorotyntsev, Electrochim. Acta 47 (2002) 2071.

54. J. Jamnik, J. Maier, and S. Pejovnik, Electrochim. Acta 44 (1999) 4139.

55. J. Jamnik and J. Maier, J. Electrochem. Soc. 146 (1999) 4183.

56. J. Jamnik and J. Maier, Phys.Chem. Chem. Phys. 3 (2001) 1668.

57. J. Jamnik, Solid State Ionics 157 (2003) 19.

58. Y.-J. Kim, Corrosion 51 (1995) 849.

59. Y.-J. Kim, Corrosion 55 (1999) 81.

60. T. Kobayashi and D. A. Shockey, FRASTA: A new way to analyze fracture surfaces, Advanced Materials and Processes, 1991, Vol. 140 (5).

61. T. Kobayashi and D. A. Shockey, Environmentally accelerated cyclic crack growth mechanisms in reactor steel determined from fracture surface topography, Proceedings of the Symposium Synergism of Microstructure, Mechanisms and Mechanics in Fracture, AIME Annual Meeting, Los Angeles, CA, 1984.

62. T. Kobayashi and D. A. Shockey, Computational reconstruction of environmentally accelerated cyclic crack growth in reactor steels, Proceedings of the National Association of Corrosion Engineers Annual Meeting, New Orleans, LA, 1989, Paper No. 563.

63. G. Schmidt and T. Kobayashi, Application of new surface analysis methods to pipeline stress corrosion cracking, International Gas Research Conference, Orlando FL, 1992.

64. T. Kobayashi, D. A. Shockey, G. Ogundele, D. D. McNabb, and D. Sidey, Deducing crack history in an aged boiler tube from fracture surface topography, Journal of Testing and Evaluation, 1994, 22 (4), p. 309. 
65. D.A. Shockey, J. H. Giovanola, T. Kobayashi, and C. G. Schmidt, New technologies for characterizing aging processes in infrastructure materials, Proceedings of the ASME/JSME Conference on Pressure Vessels \& Piping, Honolulu, Hawaii, 1995.

66. T. Kobayashi and D.A. Shockey, Advan. Mater. Processes, 140 (1991).

67. P.M. Yuzawich and C. W. Hughes, Prac. Metall. 15 (1978) 184-194.

68. B.A. Boukamp, J. Electrochem. Soc. 142 (1995) 1885.

69. Ford, F.P., Mechanisms of Environmentally-Assisted Cracking. Int. J. Pressure Vessels Pip. 40, 343-362 (1989).

70. Parkins, R.N., Current Understanding of Stress-Corrosion Cracking. JOM, December, 12-19 (1992).

71. Turnbull, A., Modelling of environment assisted cracking. Corrosion Sci. 34 (6), 921-960 (1993).

72. Galvele, J.R., Electrochemical Aspects of Stress Corrosion Cracking. In Modern Aspects of Electrochemistry, Number 27, 233-358. Edited by Ralph E. White et al., Plenum Press, New York (1995).

73. Rebak, R.B., Szklarska-Smialowska, Z., The mechanism of stress corrosion cracking of Alloy 600 in high temperature water. Corrosion Sci. 38 (6), 971-988 (1996).

74. Vogt, H., Speidel, M.O., Stress corrosion cracking of two aluminum alloys: A comparison between experimental observations and data based on modelling. Corrosion Sci. 40 (2/3), 251-270 (1998).

75. Ford, F.P., Status of Research on Environmentally Assisted Cracking in LWR Pressure Vessel Steels. J. Press. Vessel Technol.-Trans. ASME, 110, 113-128 (1988).

76. Andresen, P.L., Ford, F.P., Use of Fundamental Modeling of Environmental Cracking for Improved Design and Lifetime Evaluation. J. Press. Vessel Technol.-Trans. ASME, 115, 353-358 (1993).

77. Ford, F.P., Quantitative Prediction of Environmentally Assisted Cracking. Corrosion, 52 (5), 375-395 (1996).

78. Danielson, M.J., Oster, C.A., Jones., R.H., Crack tip chemistry modelling of intergranular stress corrosion cracks in Nickel containing segregated Phosphorus and Sulphur. Corrosion Sci. 32 (1), 1-21 (1991).

79. Macdonald, D.D., Urquidi-Macdonald, M., 1991. A coupled environment model for stress corrosion cracking in sensitized Type 304 stainless steel in LWR environments. Corrosion Sci. 32 (1), 51-81.

80. Simonen E.P., Jones, R.H., Windisch, C.F., A transport model for characterizing crack tip chemistry and mechanics during stress corrosion cracking. In New Techniques for Characterizing Corrosion and Stress Corrosion, 141-160. Edited by R.H. Jones and D.R. Baer, The Minerals, Metals \& Materials Society (1996).

81. Turnbull, A., Modelling of Crack Chemistry in Sensitized Stainless Steel in Boiling Water Reactor Environments. Corrosion Sci. 39 (4), 789-805 (1997).

82. Engelhardt, G., Urquidi-Macdonald, M., Macdonald, D.D., A Simplified Method for Estimating Corrosion Cavity Growth Rates. Corrosion Sci. 39 (3), 419-441 (1997). 
83. Engelhardt, G.R., Macdonald, D.D., Urquidi-Macdonald, M., Development of Fast Slgorithms for Estimating Stress Corrosion Crack Growth Rate. Corrosion Sci. 41, 22672302 (1999).

84. Chen, G.S., Wan, K.-C., Gao, M., Wei, R.P., Flournoy, T.H., Transition from Pitting to Fatigue Crack Growth - Modeling of Corrosion Fatigue Crack Nucleation in a 2024-T3 Aluminum Alloy. Mater. Sci. Eng. A - Struct. 219, 126-132 9 (1996).

85. Rokhlin, S.I., Kim, J.-Y., Nagy, H., Zoofan, B., Effect of pitting corrosion on fatigue crack initiation and fatigue life. Eng. Fract. Mech. 62 (4/5), 425-444 (1999).

86. Pao, P.S., Gill, S.J., Feng, C.R., On fatigue crack initiation from corrosion pits in 7075T7351 Aluminum alloy. Scripta Mater. 43, 391-396 (2000).

87. Murtaza, G., Akid, R., Empirical corrosion fatigue life prediction models of a high strength steel. Eng. Fract. Mech. 67 (5), 461-474 (2000).

88. Sankaran, K.K., Perez, R., Jata, K.V., Effects of pitting corrosion on the fatigue behavior of aluminum alloy 7075-T6: modeling and experimental studies. Mater. Sci. Eng. A Struct. 297, 223-229 (2001).

89. Kussmaul, K., Blind, D., Läpple, V., New observations on the crack growth rate of low alloy nuclear grade ferritic steels under constant active load in oxygenated high temperature water. Nucl. Eng. Des. 168, 53-75 (1997).

90. Jivkov, A., Strain-Assisted Corrosion Cracking and Growth Rate Inhibitors. Ph.D. Thesis. ISRN LUTFD2/TFME (2001).

91. Heldt, J., Seifert, H.P., Stress corrosion cracking of reactor pressure vessel steels under boiling water reactor conditions. In Proceedings of the Ninth International Symposium on Environmental Degradation of Materials in Nuclear Power Systems-Water Reactors, 901-910. Edited by F.P. Ford et al., The Minerals, Metals \& Materials Society (1999).

92. Blind, D., Huttner, F., Wunsche, A., Kuster, K., Seifert, H.P., Heldt, J., Roth, A., Karjalainen-Roikonen, P., Ehrnsten, U., European Round Robin on Constant Load EAC Tests of Low Alloy Steel under BWR Conditions. In Proceedings of the Ninth International Symposium on Environmental Degradation of Materials in Nuclear Power Systems-Water Reactors, 911-919. Edited by F.P. Ford et al., The Minerals, Metals \& Materials Society (1999).

93. Chopra, O.K., Chung, H.M., Kassner, T.F., Park, J.H., Shack, W.J., Zhang, J., Brust, F.W., Dong, P., Current research on environmentally assisted cracking in light water reactor environments. Nucl. Eng. Des. 194, 205-223 (1999).

94. Heldt, J., Seifert, H.P., Stress corrosion cracking of low-alloy, reactor-pressure vessel steels in oxygenated, high-temperature water. Nucl. Eng. Des. 206, 57-89 (2001). 